Prepared in cooperation with the Metro Wastewater Reclamation District

\title{
Water Quality at a Biosolids-Application Area near Deer Trail, Colorado, 1993-1999
}

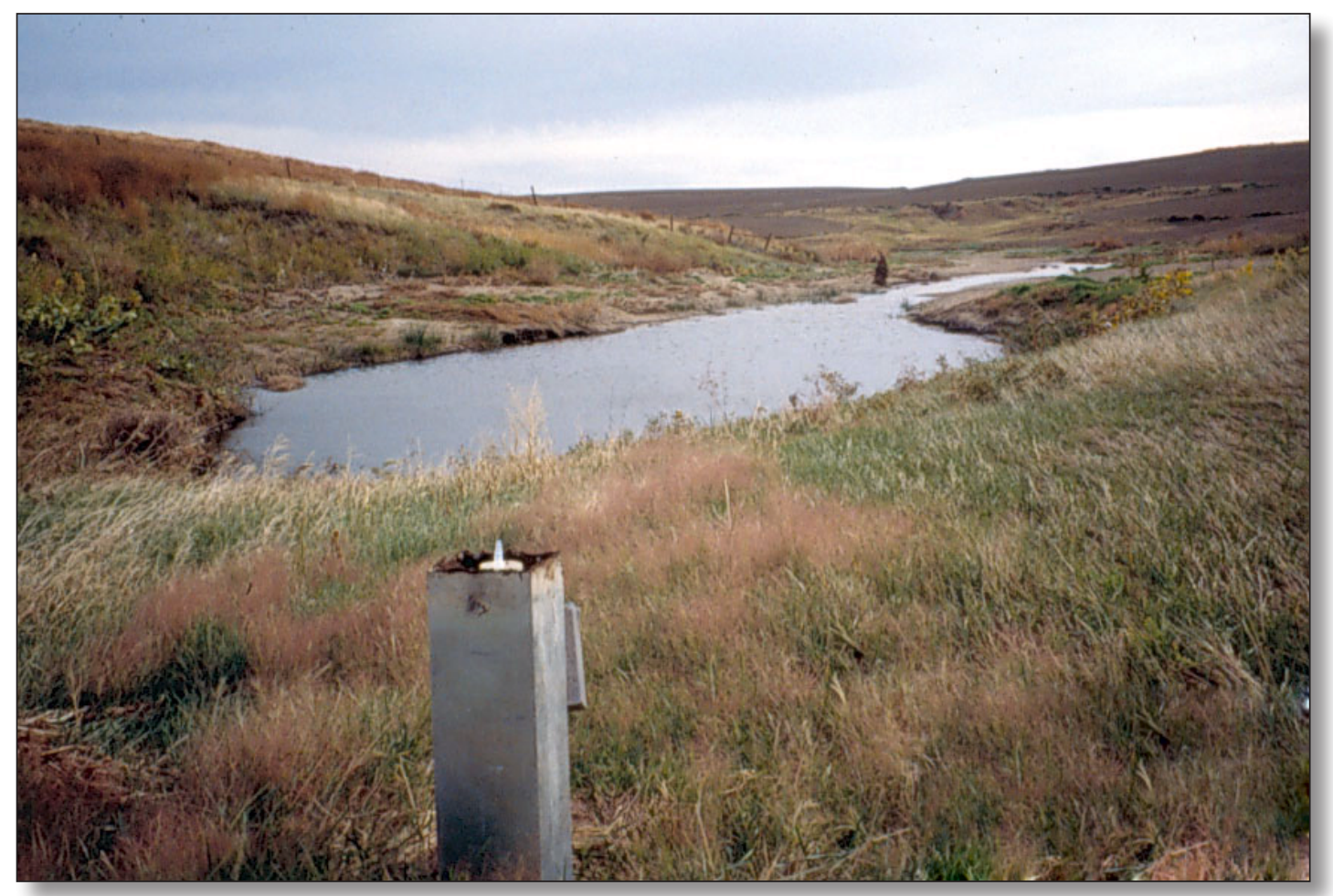

Scientific Investigations Report 2013-5074 
Cover. U.S. Geological Survey monitoring well D30 and surface-water grab-sample location S4 on October 3, 1996. 


\section{Water Quality at a Biosolids-Application Area near Deer Trail, Colorado, 1993-1999}

By Tracy J.B. Yager

Prepared in cooperation with the Metro Wastewater Reclamation District

Scientific Investigations Report 2013-5074 


\title{
U.S. Department of the Interior SALLY JEWELL, Secretary
}

\section{U.S. Geological Survey Suzette M. Kimball, Acting Director}

\author{
U.S. Geological Survey, Reston, Virginia: 2014
}

For more information on the USGS - the Federal source for science about the Earth, its natural and living resources, natural hazards, and the environment, visit http://www.usgs.gov or call 1-888-ASK-USGS.

For an overview of USGS information products, including maps, imagery, and publications, visit http://www.usgs.gov/pubprod

To order this and other USGS information products, visit http://store.usgs.gov

Any use of trade, firm, or product names is for descriptive purposes only and does not imply endorsement by the U.S. Government.

Although this information product, for the most part, is in the public domain, it also may contain copyrighted materials as noted in the text. Permission to reproduce copyrighted items must be secured from the copyright owner.

Suggested citation:

Yager, T.J.B., 2014, Water quality at a biosolids-application area near Deer Trail, Colorado, 1993-1999: U.S. Geological

Survey Scientific Investigations Report 2013-5074, 125 p., http://dx.doi.org/10.3133/sir20135074.

ISSN 2328-0328 (online) 


\section{Contents}

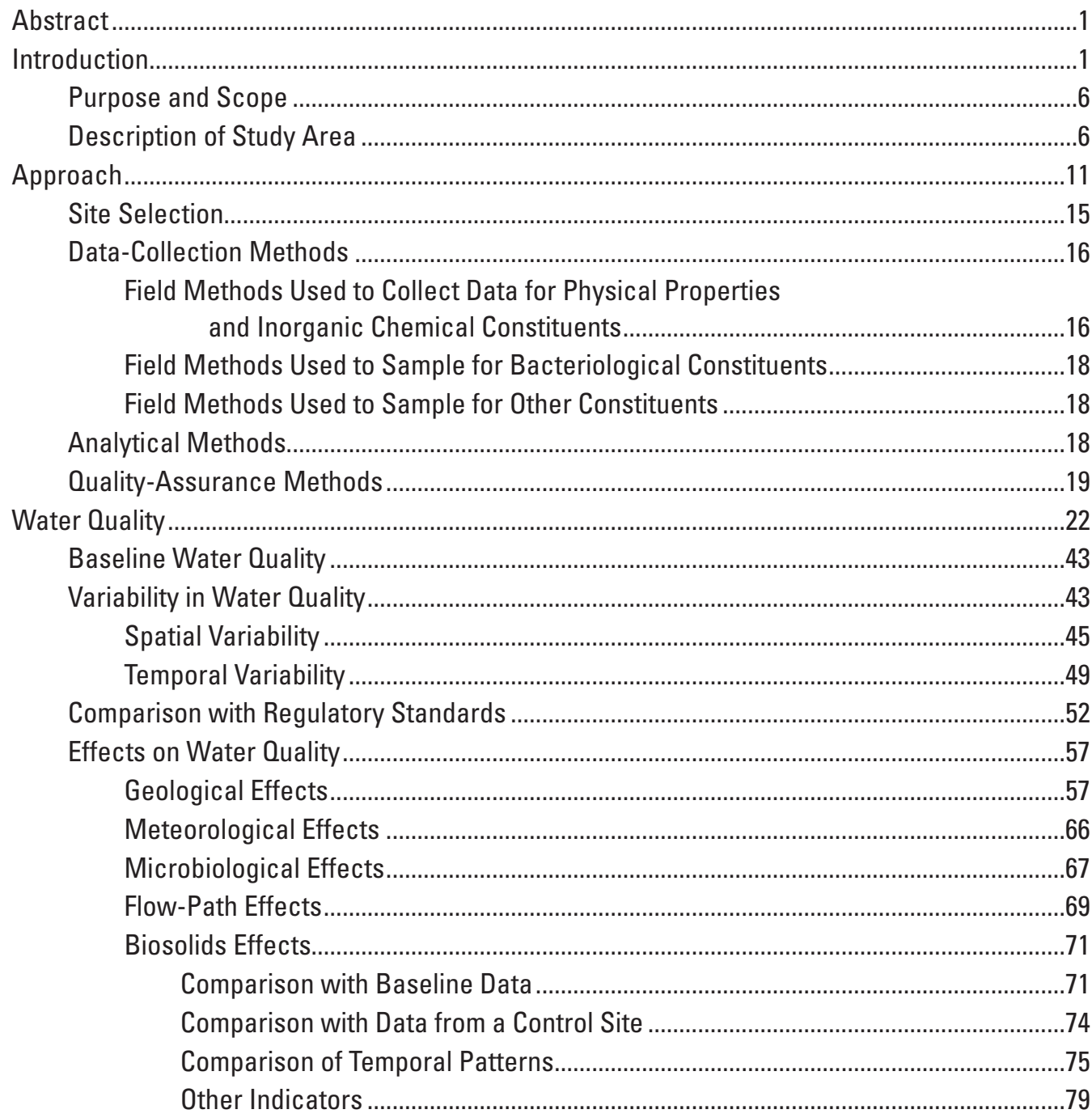

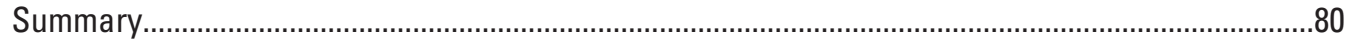

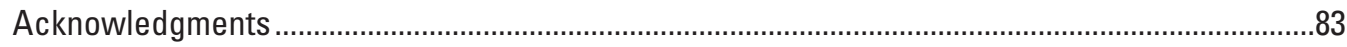

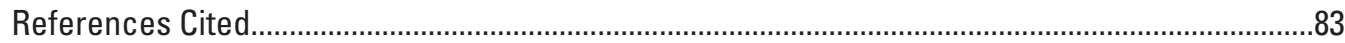

Appendix 1. Information for U.S. Geological Survey monitoring wells near Deer Trail,

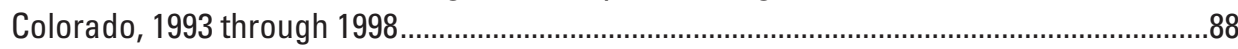

Appendix 2. Chemical symbols and abbreviations used in reporting chemical data in the appendixes.......................................................................................................................90

Appendix 3. Physical properties and major-ion data for replicate water-quality samples collected near Deer Trail, Colorado, 1993 through 1998 ......................................................91

Appendix 4. Nutrient data for replicate water-quality samples collected near Deer Trail, Colorado, 1993 through 1998

Appendix 5. Trace-element data for replicate water-quality samples collected near Deer Trail, Colorado, 1993 through 1998.

Appendix 6. Codes used in reporting information and chemical data (appendix 7) for blank water-quality samples collected near Deer Trail, Colorado, 1993 through 1999. 
Appendix 7. Information and chemical data for blank water-quality samples collected near Deer Trail, Colorado, 1993 through 1999

Appendix 8. Performance-evaluation data from the U.S. Geological Survey Standard Reference Program for the Metro Wastewater Reclamation District Laboratory (1993-1998) and the U.S. Geological Survey National Water Quality Laboratory (1993-1999)...

Appendix 9. Geochemical modeling results for well D6 using NETPATH for groundwater samples collected near Deer Trail, Colorado, 1993 to 1999

\section{Figures}

1. Map showing location of the study area near Deer Trail, Colorado

2. Map showing study area and U.S. Geological Survey sampling sites on original (1993-1995) Metro Wastewater Reclamation District property near Deer Trail, Colorado, 1993 through 1999

3. Map showing location of U.S. Geological Survey Expanded Monitoring Program sites near Deer Trail, Colorado, 1999.

4. Photograph showing biosolids after application to a field near Deer Trail, Colorado ............6

5. Map showing Metro Wastewater Reclamation District biosolids-application areas near Deer Trail, Colorado

6. Map showing drainage basins in the study area......

7. Schematic diagram showing summary of the U.S. Geological Survey sampling method used to collect groundwater-quality samples near Deer Trail, Colorado, late 1995 through 1999.

8. Map showing baseline water quality for selected constituents in groundwater and surface-water samples collected in 1993 at the Metro Wastewater Reclamation District property..

9. Trilinear diagrams showing water type for $(A)$ drainage basin, $(B)$ site type, $(C)$ well depth, and $(D)$ water-composition groupings near Deer Trail, Colorado, based on median data for 1993-1998

10. Map showing groundwater major-ion water-composition groups (figure 9D) overlaying the geologic map presented by Sharps (1980)

11. Graphs showing water-quality data for selected dissolved constituents compared with sample depth and (for groundwater samples) depth to groundwater near Deer Trail, Colorado, 1993-1998.

12. Graphs showing groundwater concentrations of nitrite plus nitrate as nitrogen over time atamonitoring wells near Deer Trail, Colorado, 1993 through 1998.

13. Graphs showing distribution of groundwater-concentration data for samples collected near Deer Trail, Colorado, compared to regulatory standards for selected constituents, 1993 through 1999.

14. Graphs showing ion plots showing milliequivalent ratios for $(A)$ gypsum, $(B)$ calcite, $(C)$ dolomite, $(D)$ halite, and $(E)$ epsomite (or another magnesiumsulfate mineral) from samples collected from selected monitoring wells near Deer Trail, Colorado, 1993 through 1998.

15. Graphs showing comparison of baseline, post-biosolids, and control-site groundwater-quality data for selected constituents in samples from all monitoring wells near Deer Trail, Colorado, 1993 through 1998.

16. Graphs showing comparison of selected water-quality constituents with biosolidsapplication data for selected sites near Deer Trail, Colorado, 1993 through 1998 


\section{Tables}

1. Biosolids applications to the study area near Deer Trail, Colorado, 1993 through 1999.........8

2. U.S. Geological Survey sampling sites near Deer Trail, Colorado, 1993 through 1999.......... 12

3. Laboratory methods used by the Metro Wastewater Reclamation District Laboratory

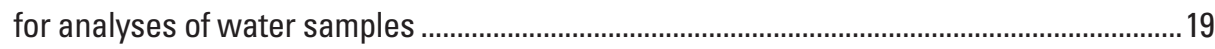

4. Laboratory methods used by the U.S. Geological Survey National Water Quality

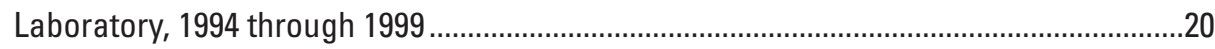

5. Water-quality data for samples collected near Deer Trail, Colorado, 1993 through 1998

6. Bacteria data for water-quality samples collected near Deer Trail, Colorado, 1993 through 1998.

7. Water-quality data for redox-indicator sampling near Deer Trail, Colorado, July 1999.

8. Summary of groundwater-quality data collected for this study near Deer Trail, Colorado, during 1993-1999, and groundwater regulatory limits

9. Chemical data for core samples collected from D31 and D33 boreholes during drilling near Deer Trail, Colorado, May 1995.

10. Concentrations of redox-sensitive constituents and terminal electron-acceptor processes for groundwater from selected wells near Deer Trail, Colorado, July 1999.

11. Groundwater information indicated by dissolved-gas sampling at selected wells near Deer Trail, Colorado, 1998.

12. Groundwater information indicated by chlorofluorocarbon sampling at selected wells near Deer Trail, Colorado, 1998

\section{Conversion Factors, Abbreviations, and Datums}

\begin{tabular}{|c|c|c|}
\hline Multiply & By & To obtain \\
\hline \multicolumn{3}{|c|}{ Length } \\
\hline foot $(\mathrm{ft})$ & 0.3048 & meter $(\mathrm{m})$ \\
\hline inch & 2.54 & centimeter $(\mathrm{cm})$ \\
\hline kilometer (km) & 0.6214 & mile (mi) \\
\hline micrometer (micron) & 0.00003937 & inch (in.) \\
\hline mile (mi) & 1.609 & kilometer $(\mathrm{km})$ \\
\hline \multicolumn{3}{|c|}{ Area } \\
\hline acre & 0.004047 & square kilometer $\left(\mathrm{km}^{2}\right)$ \\
\hline section ( 640 acres or 1 square mile) & 259.0 & square hectometer $\left(\mathrm{hm}^{2}\right)$ \\
\hline square mile $\left(\mathrm{mi}^{2}\right)$ & 2.590 & square kilometer $\left(\mathrm{km}^{2}\right)$ \\
\hline \multicolumn{3}{|c|}{ Volume } \\
\hline gallon (gal) & 3.785 & liter (L) \\
\hline liter (L) & 0.2642 & gallon (gal) \\
\hline milliliter $(\mathrm{mL})$ & 0.002113 & pint $(\mathrm{pt})$ \\
\hline \multicolumn{3}{|c|}{ Mass } \\
\hline milligram (mg) & 35.27 & ounce, avoirdupois (oz) \\
\hline pound, avoirdupois (lb) & 0.4536 & kilogram $(\mathrm{kg})$ \\
\hline ton, short $(2,000 \mathrm{lb})$ & 0.9072 & megagram $(\mathrm{Mg})$ \\
\hline ton, long (2,240 lb) & 1.016 & megagram $(\mathrm{Mg})$ \\
\hline \multicolumn{3}{|c|}{ Application } \\
\hline pound per acre (lb/acre) & 1.121 & kilogram per hectare \\
\hline
\end{tabular}


Temperature in degrees Celsius $\left({ }^{\circ} \mathrm{C}\right)$ may be converted to degrees Fahrenheit $\left({ }^{\circ} \mathrm{F}\right)$ as follows:

$$
{ }^{\circ} \mathrm{F}=\left(1.8 x^{\circ} \mathrm{C}\right)+32
$$

Vertical coordinate information is referenced to the National Geodetic Vertical Datum of 1929 (NGVD 29) or North American Vertical Datum of 1988 (NAVD 88), as noted.

Horizontal coordinate information is referenced to the North American Datum of 1983 (NAD 83).

Elevation, as used in this report, refers to distance above the vertical datum.

Specific conductance is given in microsiemens per centimeter at 25 degrees Celsius $(\mu \mathrm{S} / \mathrm{cm})$.

Concentrations of chemical constituents in water are given either in milligrams per liter $(\mathrm{mg} / \mathrm{L})$, micrograms per liter $(\mu \mathrm{g} / \mathrm{L})$, or milliequivalents per liter (meq/L); parts per million (PPM) is equivalent to milligrams per liter, and parts per billion (PPB) is equivalent to micrograms per liter.

Concentrations of chemical constituents in gas are given either in milligrams per liter (mg/L), or nanomoles per liter ( $\mathrm{nmol} / \mathrm{L})$.

Concentrations of chemical constituents in solids are given in parts per million (PPM), which is equivalent to milligrams per kilogram $(\mathrm{mg} / \mathrm{kg})$ or micrograms per gram $(\mu \mathrm{g} / \mathrm{g})$, or in percent composition by mass (\%).

\section{Additional Abbreviations}

$\begin{array}{ll}\text { Metro District } & \text { Metro Wastewater Reclamation District } \\ \text { NWOL } & \text { National Water Quality Laboratory } \\ \text { USGS } & \text { U.S. Geological Survey } \\ \text { CFC } & \text { Chlorofluorocarbons } \\ \text { MRL } & \text { Minimum reporting level } \\ \text { TEAP } & \text { Terminal electron-acceptor process }\end{array}$




\title{
Water Quality at a Biosolids-Application Area near Deer Trail, Colorado, 1993-1999
}

\author{
By Tracy J.B. Yager
}

\section{Abstract}

The Metro Wastewater Reclamation District (Metro District) in Denver, Colo., applied biosolids resulting from municipal sewage treatment to farmland in eastern Colorado beginning in December 1993. In mid-1993, the U.S. Geological Survey in cooperation with the Metro District began monitoring water quality at the biosolids-application area about 10 miles east of Deer Trail, Colo., to evaluate baseline water quality and the combined effects of natural processes, land uses, and biosolids applications on water quality of the biosolidsapplication area. Water quality was characterized by baseline and post-biosolids-application sampling for selected inorganic and bacteriological constituents during 1993 through 1998, with some additional specialized sampling in 1999. The study included limited sampling of surface water and the unsaturated zone, but primarily focused on groundwater.

The baseline data indicate that major-ion, nutrient, trace-element, and bacteria sources other than biosolids were present in the study area and that water in the study area was of variable quality before biosolids ever were applied. Large concentrations of chloride, sulfate, nitrate, iron, and manganese were detected in some baseline samples. Concentrations of other major ions, phosphorus, cadmium, chromium, nickel, and fecal streptococcus bacteria also were detected above the minimum reporting level in baseline samples.

Variability in water quality of the study area was evident from the baseline data as well as from the data collected after biosolids applications began. The hydrology of the study area likely accounts for some of the variability in water quality; groundwater recharged preferentially from ponds and through desiccation cracks and coarse-grained deposits and outcrops, not uniformly through the unsaturated zone beneath all fields. Spatial variability was largest for concentrations of sulfate, nitrate, iron, and manganese. Soil pore-water data indicated that chloride, nitrate, and copper reservoirs in the upper 3 feet of the unsaturated zone could eventually migrate to the saturated zone. Temporal variability was largest for concentrations of nitrate, iron, and manganese and fecal streptococcus bacteria counts. Of the limited analytes considered for this study, nitrate increased in concentration the most consistently over time.
Groundwater samples from the study area exceeded various Colorado regulatory standards, both in baseline samples and in post-biosolids-treatment samples. Concentrations of constituents in the groundwater exceeded Colorado standards for nitrate, chromium, iron, manganese, and nickel concentrations. Baseline groundwater concentrations of chloride, sulfate, nitrate, iron, and manganese in samples from some sites exceeded Colorado regulatory standards before biosolids applications to the study area began, sometimes by a large margin.

In general, biosolids applications may have affected water quality in parts of the study area during 1993-1998 through increases in nitrate and possibly a few other constituents, particularly where groundwater was oxic. However, increased nitrate concentrations were not widespread throughout the study area during the $1-5$ years after biosolids applications began. Microbial processes in oxygen-depleted shallow groundwater have kept nitrate concentrations small and could reduce additional nitrogen inputs to the groundwater. For most water-quality constituents, any effects from biosolids on water quality of the study area during 1993-1998 were obscured by high variation in concentrations, and the effects of biosolids applications on water quality of the study area during 1993-1999 were less than natural geological or microbiological effects.

\section{Introduction}

Municipal sewage from the Denver area was treated at the Metro Wastewater Reclamation District (Metro District) in Denver, Colo. (fig. 1). Biosolids are solid organic matter recovered from a sewage-treatment process that met State and Federal regulatory criteria for a beneficial use such as soil amendment or fertilizer (Colorado Department of Public Health and Environment, 1998). In 1993, the Metro District acquired farmland and rangeland (about 15 square miles, $\mathrm{mi}^{2}$; fig. 2) on the eastern plains of Colorado in Arapahoe and Elbert Counties about 10 miles (mi) east of Deer Trail, Colo. (fig. 1). During December 1993 through 1999 (1993-1999), the Metro District applied biosolids as a fertilizer and soil amendment to their farmland property near Deer Trail. The biosolids were trucked about $75 \mathrm{mi}$ east from Denver to the Metro District property (fig. 1) and were applied to 


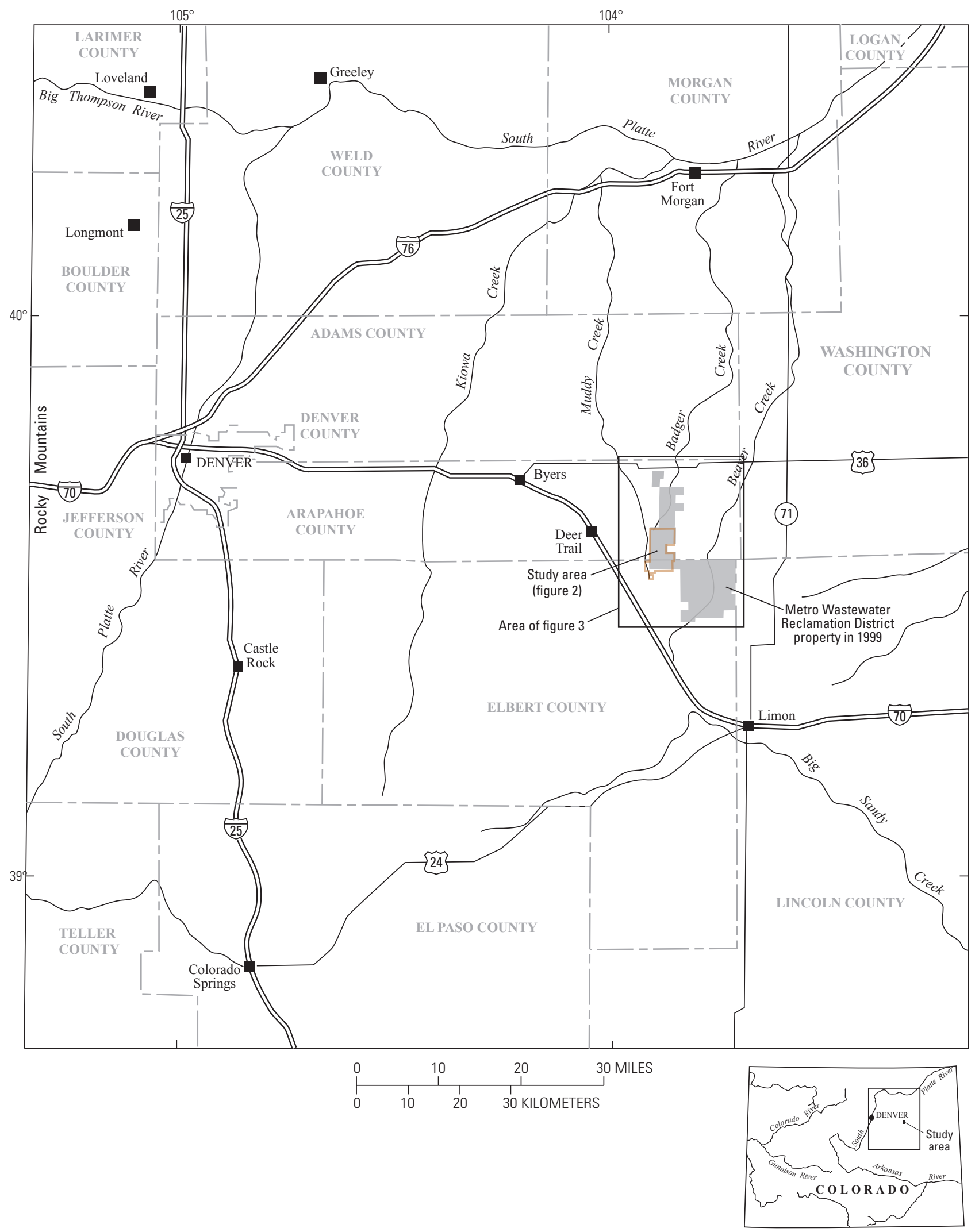

Figure 1. Location of the study area near Deer Trail, Colorado. 


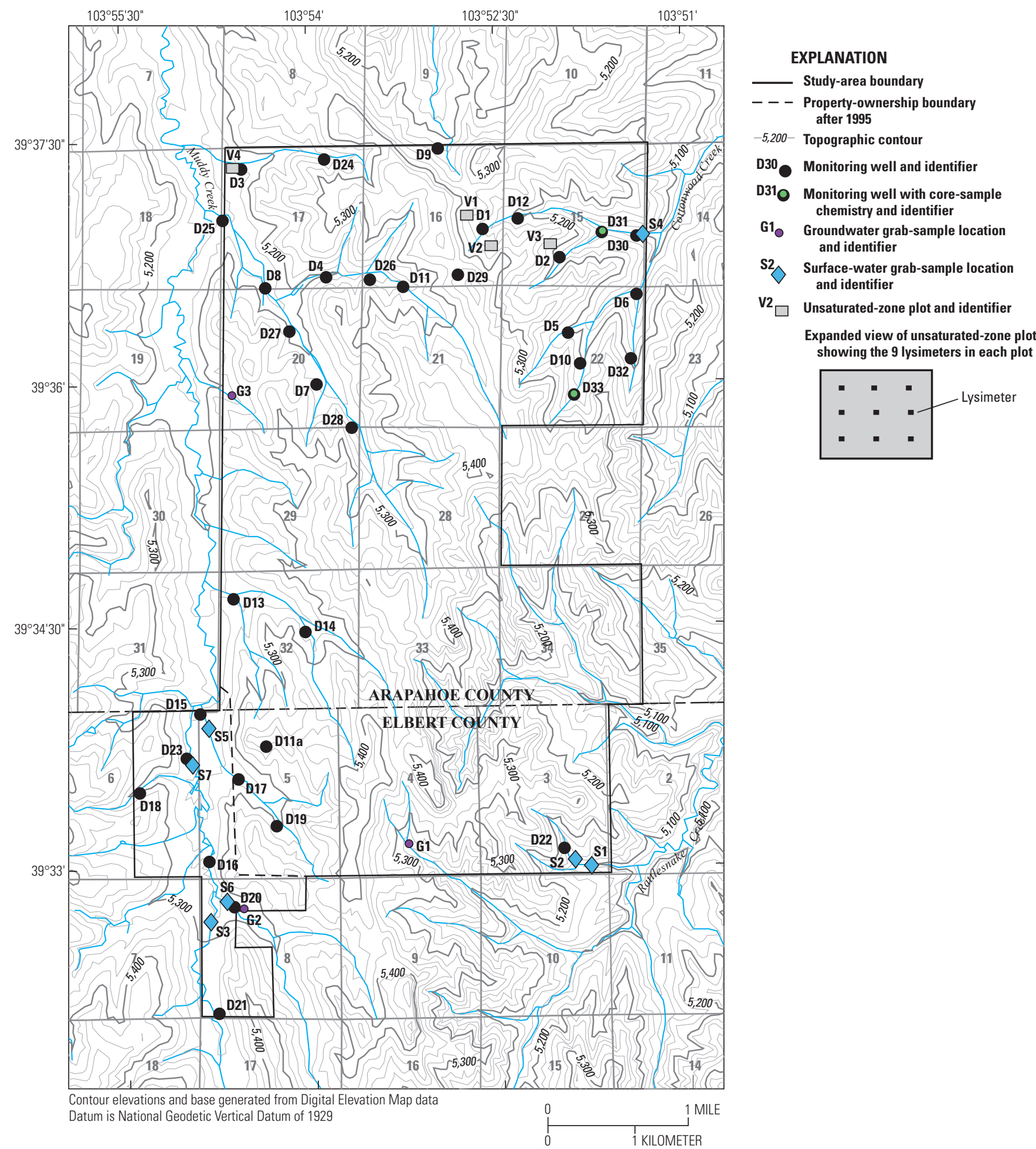

Figure 2. Study area and U.S. Geological Survey sampling sites on original (1993-1995) Metro Wastewater Reclamation District property near Deer Trail, Colorado (now part of the Central property), 1993 through 1999. 
nonirrigated farmland. In 1995, the Metro District traded some of the first property and acquired additional property in the same area. The revised boundaries of the original property became known as the Metro District Central property (fig. 3). The additional properties consisted of about $14.5 \mathrm{mi}^{2}$ known as the North property and about $50 \mathrm{mi}^{2}$ known as the South property (fig. 3). In 1999, the three Metro District properties together (North, South, and Central), known as the METROGRO Farm, encompassed almost $70 \mathrm{mi}^{2}$ of farmland in Arapahoe and Elbert Counties (fig. 3). The Metro District properties continued to be farmed after the applications of biosolids began.

Animal waste related to grazing wildlife and domestic livestock, as well as farming applications of pesticides and fertilizers (including biosolids), can affect the quality of water in the unsaturated zone, ponds or streams, and alluvial and bedrock aquifers (Freeze and Cherry, 1979). Water quality can be affected directly by contaminated recharge water or by infiltration of water through contaminated soils or sediments (remobilization). Water quality can be affected indirectly by tilling that mobilizes or mixes subsurface chemical constituents or by contributions to natural processes such as nitrification. Contaminated shallow groundwater or surface water could contaminate other aquifers (such as bedrock water-supply aquifers or alluvial aquifers), other surface-water bodies (ponds or streams), or streambed sediments.

Past studies indicated that various land-disposal methods for human sewage products can increase concentrations of inorganic and organic constituents in water at the disposal site (Berti and Jacobs, 1996 and 1998; Eljarrat and others, 1997; Moolenaar and Beltrami, 1998; Sloan and others, 1998; Steenhuis and others, 1999). Robson (1977), Gaggiani (1991), Johncox and Gaggiani (1991), Tindall and others (1994), and Lull and Gaggiani (1996) all reported increased concentrations of selected inorganic constituents in groundwater or surface water at sewage-disposal sites in Colorado. However, biosolids applications differ from pit disposal of other sewage products because biosolids must meet additional regulatory criteria and application rates based on growing vegetation that is planned to assimilate nitrogen, phosphorus, and trace elements as plant nutrients.

Biosolids applications to the study area began in late 1993 and continue in all but the southwest part of the study area through the present (October 1, 2012). Metro District staff transported and applied the biosolids, and only biosolids from the Metro District treatment plant were applied to the study area. Biosolids were applied according to agronomic loading rates through a process permitted by the State of Colorado. The agronomic loading rates resulted in biosolids lightly broadcast on the fields, not thickly laid (fig. 4). Metro District biosolidsapplication areas in the vicinity of the study area are shown in figure 5. Detailed information about each biosolids application to these areas during 1993-1999 is listed in table 1. Landapplied biosolids had to meet regulations (Colorado Department of Public Health and Environment, 1998; U.S. Environmental Protection Agency, 1993); otherwise, agronomic loading rates might be exceeded and the area could become overloaded with nitrogen and trace elements. Soil quality either can be improved by biosolids applications through increased nutrients and organic matter or degraded through accumulation of excessive nutrients or other constituents.

Water quality at a biosolids-application site may not represent effects from biosolids just because biosolids were present at that site. Many of the chemical constituents used as indicators of biosolids contamination (such as trace elements) also could be contributed by geologic materials (Drever, 1988). Other water-quality constituents (such as nutrients and organic compounds) attributed to biosolids also could be contributed by other farming practices (Freeze and Cherry, 1979) or by livestock grazed on the property. Water quality is not just the result of chemicals added to the land surface but also is affected by dissolution, precipitation, reaction, and transport of natural and anthropogenic chemical constituents along the surface and in the subsurface, depending on the flow path of the water. Water quality can be affected by the hydrology, weather, and geology of a site. The chemical constituents of the geologic materials interact in the presence of water through a variety of natural processes. The chemistry of water in the unsaturated zone, ponds, streams, and aquifers at a biosolids-application site will be determined by complicated geochemical reactions affected by geology, hydrology, microbiology, land use, and anthropogenic applications. Thus, an interpretation of the contribution of biosolids to water quality requires an understanding of the geology, hydrology, land-use activities, and baseline water quality of the site.

In mid-1993, the U.S. Geological Survey (USGS) in cooperation with the Metro District began monitoring hydrology and water quality on the Metro District property near Deer Trail. This study was done to evaluate baseline water quality and the combined effects of natural processes, land uses, and biosolids applications on water quality of the biosolidsapplication area. The study area consisted of the 1993 Metro District property (fig. 2). Hydrology was monitored from 1993 through 1998 (1993-1998) by measuring groundwater levels and precipitation. Water quality was monitored from 1993-1998 by sampling for selected inorganic and bacteriological constituents, with some additional specialized sampling in 1999. The study included limited sampling of surface water and the unsaturated zone, but primarily focused on the sampling of groundwater, which was more abundant. In 1999, the USGS also began the Expanded Monitoring Program at the same location (conducted during 1999-2004), in cooperation with the Metro District and the North Kiowa Bijou Groundwater Management District, that built on the earlier USGS monitoring program but included all three Metro District properties (fig. 3) and expanded monitoring components. As part of the Expanded Monitoring Program, waterquality data were collected, the geology of the area was evaluated to identify aquifer materials, and hydrogeologic structure maps were prepared. The results of monitoring hydrology in the study area during 1993-1998 were reported by Yager and Arnold (2003), along with geologic information and hydrogeologic structure maps for the Expanded Monitoring Program (the area shown in fig. 3). 


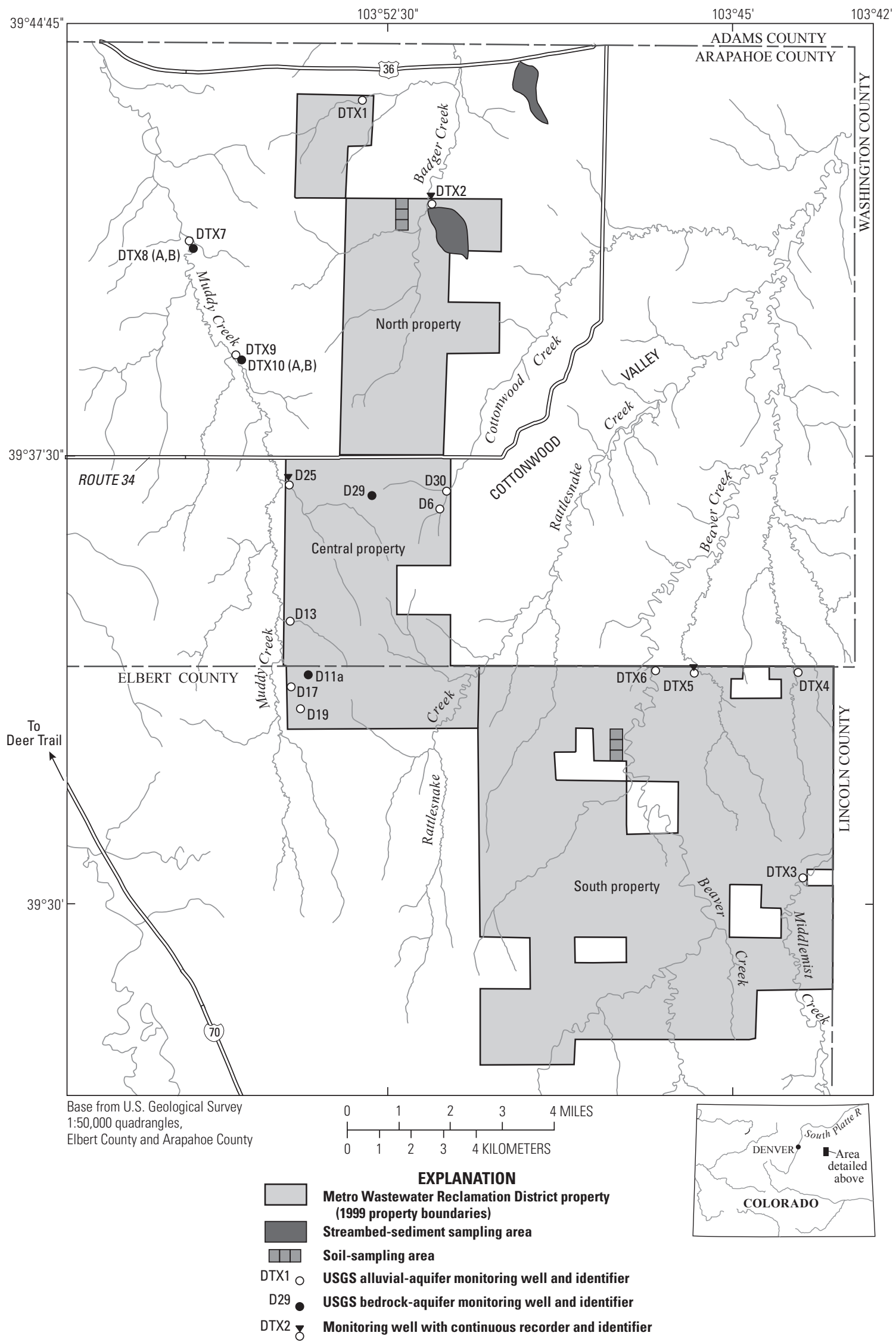

Figure 3. Location of U.S. Geological Survey Expanded Monitoring Program sites near Deer Trail, Colorado, 1999. [Base from U.S. Geological Survey, 1976; U.S. Geological Survey, 1980a] 


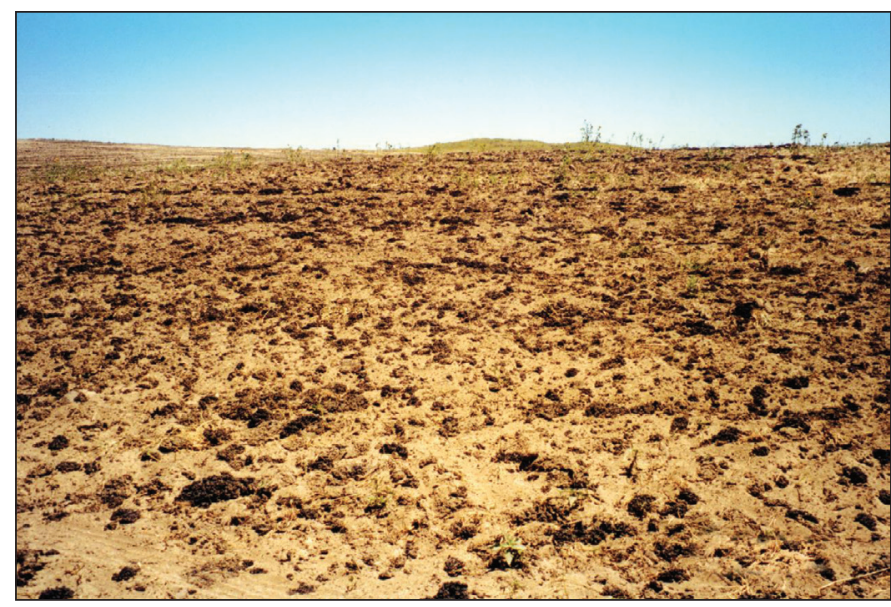

Figure 4. Biosolids (dark clumps) after application to a field near Deer Trail, Colorado. Photograph by U.S. Geological Survey.

\section{Purpose and Scope}

The purpose of this report is to present the results of water-quality monitoring done by the USGS near Deer Trail, Colo., during 1993-1999 as part of the first USGS study related to biosolids applications in that area. The report includes water-quality data for alluvial and bedrock groundwater in addition to limited data for surface water and the unsaturated zone. Chemical data for core samples from two boreholes in the study area also are included. The focus of the report is an interpretive discussion of water quality for the study area. The report does not include the data and interpretations for 1999 from the Expanded Monitoring Program; these results were reported by Stevens and others (2003) and Yager and others (2004b).

The report includes additional, related information in various appendixes. Detailed monitoring-well information such as well-completion data are listed in appendix 1. Chemical symbols and abbreviations used in reporting qualitycontrol data are listed in appendix 2. Quality-control data for the water-quality samples collected during 1993-1999 are listed in appendixes 3 through 8 . The results of geochemical modeling done for groundwater quality at one well are included as appendix 9 .

\section{Description of Study Area}

The study area (described in more detail in Yager and Arnold, 2003; Yager and others, 2004b) was located on the eastern plains of Colorado about 10 mi east of Deer Trail (fig.1). The study area was on the eastern margin of the Denver Basin, a bowl-shaped sequence of sedimentary rocks that was formed in an ocean or near-ocean environment. The surficial geology of the study area consists of interbedded shale, siltstone, and sandstone, which may be overlain by clay, windblown silt and sand, or alluvial sand and gravel
(Sharps, 1980; Major and others, 1983; Robson and Banta, 1995). The primary water-supply aquifer in the study area during 1993-1999 was the Laramie-Fox Hills aquifer, which is a bedrock aquifer that ranged from 0 to about 200 feet ( $\mathrm{ft}$ ) in thickness in the study area and is the bottom aquifer in the Denver Basin aquifer sequence (Robson, 1981; Robson and Banta, 1995; Yager and Arnold, 2003). Multiple alluvial aquifers were present in the study area during 1993-1999. Depth to groundwater below land surface at sampling locations ranged from a few feet to about $100 \mathrm{ft}$. The alluvial aquifers were associated with the surficial drainage network and contained water of variable quality, were of limited extent, and generally yielded little water (Stevens and others, 2003; Yager and Arnold, 2003). Parts of four drainage basins comprise the study area: Badger Creek, Cottonwood Creek, Rattlesnake Creek, and Muddy Creek (fig. 6); the Cottonwood Creek and Rattlesnake Creek drainage basins are part of the Beaver Creek drainage basin (Seaber and others, 1987). The entire study area is within the South Platte River drainage basin; all streams in this area drain northward to the South Platte River (fig. 1; Yager and Arnold, 2003). Short segments of some of the streams were intermittent, but in general, the streams during 1993-1999 were ephemeral and flowed only after storms. No surface water flowed off the Metro District properties except after storms. Surface water predominantly was in the form of ponds. Most ponds in the area were created by water-detention structures. The climate was semi-arid; less than 20 inches of precipitation usually was received each year (Yager and Arnold, 2003). Most of the precipitation occurred as rainfall in spring and in late summer. Soils in the area generally were sandy or loamy on flood plains and stream terraces, clayey to loamy on gently sloping to rolling uplands, and sandy and shaley on steeper uplands (Larsen and others, 1966; Larsen and Brown, 1971).

Land use in the study area historically was rangeland or cropland and pasture (U.S. Geological Survey, 1980b). Some petroleum exploration was done in the area (Drew and others, 1979), but no oil or gas production took place on the Metro District properties during 1993-1999 (N. Crews, Metro Wastewater Reclamation District, written commun., April 13, 2011). Land in the study area was used as rangeland or cropland during 1993-1999. Cattle and sheep were the primary domesticated animals grazing the area, although no concentrated animal-feeding operations were located on the Metro District properties during 1993-1999. Winter wheat was the primary crop during 1993-1999; farmland was not irrigated. Herbicides and other chemicals were applied to the study area for farming and weed-control purposes (B. Patterson, Metro Wastewater Reclamation District, written commun., December 6, 1999). Pesticides and other fertilizers also may have been applied to the Metro District properties historically, but little information was available about these historical applications. Biosolids were applied as the only fertilizer and soil amendment on the Metro District properties during the study. 


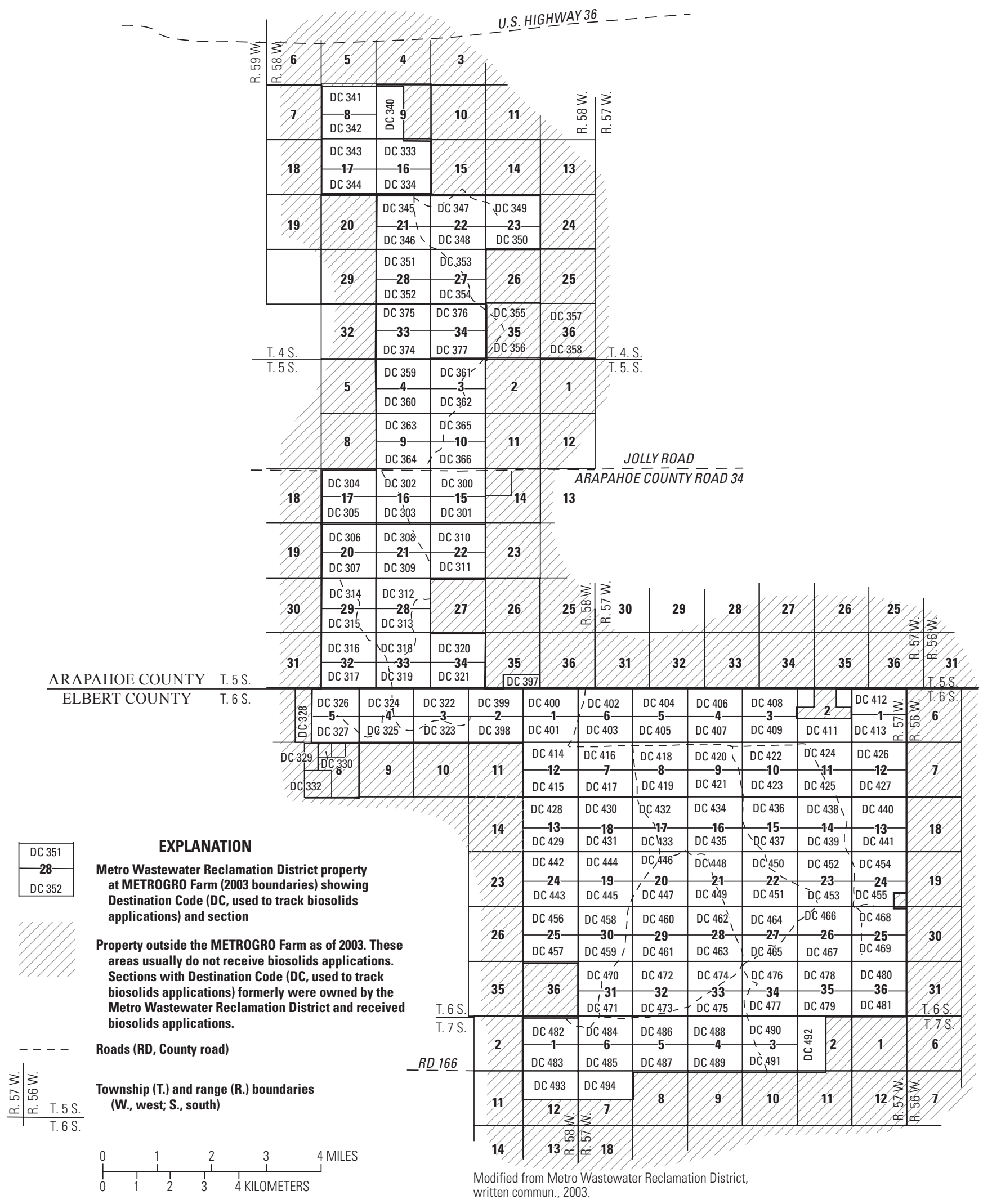

Figure 5. Metro Wastewater Reclamation District biosolids-application areas near Deer Trail, Colorado. 
Table 1. Biosolids applications to the study area near Deer Trail, Colorado, 1993 through 1999.

[All information provided by the Metro Wastewater Reclamation District; DC, destination code; wT, wet tons; dT, dry tons; Cake, Table 3 Class B biosolids; --, no information available; lb, pound; MAC, biosolids ammended with wood fiber; incorporated, biosolids are pushed down into the soil 4 to 8 inches after application by the tines of a rotating implement pulled by a tractor]

\begin{tabular}{|c|c|c|c|c|c|c|c|c|c|c|c|c|}
\hline $\begin{array}{c}\text { DC } \\
\text { (fig. 5) }\end{array}$ & $\begin{array}{l}\text { Application } \\
\text { start date }\end{array}$ & $\begin{array}{l}\text { Application } \\
\text { stop date }\end{array}$ & $\begin{array}{c}\text { Area } \\
\text { applied, } \\
\text { acre }\end{array}$ & $\begin{array}{l}\text { Product } \\
\text { applied }\end{array}$ & $\begin{array}{c}\text { Application } \\
\text { method }\end{array}$ & $\begin{array}{l}\text { Number } \\
\text { of loads } \\
\text { applied }\end{array}$ & $\begin{array}{c}\text { Total } \\
\text { biosolids } \\
\text { applied, } \\
\text { wT }\end{array}$ & $\begin{array}{c}\text { Total } \\
\text { biosolids } \\
\text { applied, } \\
\text { dT }\end{array}$ & $\begin{array}{l}\text { Biosolids } \\
\text { loading rate, } \\
\text { dT/acre }\end{array}$ & $\begin{array}{c}\text { Nitrogen } \\
\text { loading rate, } \\
\text { lb/acre }\end{array}$ & Crop & $\begin{array}{c}\text { Reclamation } \\
\text { project }^{1}\end{array}$ \\
\hline 301 & $12 / 8 / 1993$ & $6 / 8 / 1994$ & 286.2 & Cake & -- & 208 & 4,586 & 787 & 2.75 & 74 & Wheat & -- \\
\hline 302 & $12 / 8 / 1993$ & 7/18/1994 & 301.6 & Cake & -- & 242 & 5,329 & 886 & 2.94 & 75 & Wheat & -- \\
\hline 303 & $12 / 8 / 1993$ & 8/2/1994 & 301.6 & Cake & -- & 207 & 4,615 & 796 & 2.64 & 70 & Wheat & -- \\
\hline 309 & 3/4/1994 & $3 / 14 / 1994$ & 320.0 & Cake & -- & 202 & 4,503 & 782 & 2.44 & 66 & Wheat & -- \\
\hline 311 & $3 / 15 / 1994$ & 4/19/1994 & 320.0 & Cake & -- & 170 & 3,783 & 651 & 2.03 & 48 & Wheat & -- \\
\hline 310 & 4/20/1994 & 5/7/1994 & 299.1 & Cake & -- & 221 & 4,880 & 854 & 2.86 & 69 & Wheat & -- \\
\hline 308 & $5 / 7 / 1994$ & $5 / 27 / 1994$ & 314.0 & Cake & -- & 240 & 5,438 & 953 & 3.04 & 73 & Wheat & -- \\
\hline 300 & $6 / 12 / 1994$ & 6/24/1994 & 286.2 & Cake & -- & 206 & 4,708 & 827 & 2.89 & 75 & Wheat & -- \\
\hline 304 & $7 / 3 / 1994$ & $7 / 18 / 1994$ & 267.4 & Cake & -- & 172 & 3,814 & 650 & 2.43 & 64 & Wheat & -- \\
\hline 305 & $7 / 19 / 1994$ & $7 / 27 / 1994$ & 225.7 & Cake & -- & 168 & 3,746 & 627 & 2.78 & 62 & Wheat & -- \\
\hline 306 & 8/6/1994 & 8/13/1994 & 180.0 & Cake & -- & 114 & 2,527 & 423 & 2.35 & 53 & Wheat & -- \\
\hline 306 & 8/6/1994 & $5 / 10 / 1995$ & 232.0 & Cake & -- & 153 & 3,390 & 572 & 2.47 & 57 & Wheat & -- \\
\hline 307 & $8 / 12 / 1994$ & $8 / 13 / 1994$ & 60.0 & Cake & -- & 32 & 707 & 122 & 2.03 & 46 & Wheat & -- \\
\hline 307 & $8 / 12 / 1994$ & $5 / 31 / 1995$ & 250.0 & Cake & -- & 134 & 3,041 & 527 & 2.11 & 53 & Wheat & -- \\
\hline 312 & 8/14/1994 & 4/8/1995 & 305.0 & Cake & -- & 180 & 3,995 & 650 & 2.13 & 55 & Wheat & -- \\
\hline 323 & 10/4/1994 & 10/9/1994 & 140.0 & Cake & -- & 110 & 2,444 & 404 & 2.89 & 72 & Wheat & -- \\
\hline 322 & $10 / 10 / 1994$ & $10 / 22 / 1994$ & 245.0 & Cake & -- & 159 & 3,565 & 594 & 2.42 & 58 & Wheat & -- \\
\hline 313 & $11 / 4 / 1994$ & 4/6/1995 & 280.0 & Cake & -- & 232 & 5,113 & 807 & 2.88 & 77 & Wheat & -- \\
\hline 318 & 4/8/1995 & $6 / 29 / 1995$ & 300.0 & Cake & -- & 246 & 5,478 & 887 & 2.96 & 82 & Wheat & -- \\
\hline 319 & 4/14/1995 & 4/30/1995 & 180.0 & Cake & -- & 135 & 3,044 & 495 & 2.75 & 79 & Wheat & -- \\
\hline 324 & $5 / 1 / 1995$ & $5 / 6 / 1995$ & 135.0 & Cake & -- & 108 & 2,459 & 393 & 2.91 & 76 & Wheat & -- \\
\hline 325 & $5 / 6 / 1995$ & $5 / 20 / 1995$ & 180.0 & Cake & -- & 132 & 3,026 & 526 & 2.92 & 84 & Wheat & -- \\
\hline 315 & $5 / 23 / 1995$ & $5 / 26 / 1995$ & 95.0 & Cake & -- & 75 & 1,504 & 261 & 2.75 & 64 & Wheat & -- \\
\hline 314 & $5 / 27 / 1995$ & $5 / 30 / 1995$ & 95.0 & Cake & -- & 69 & 1,583 & 265 & 2.79 & 72 & Wheat & -- \\
\hline 316 & $5 / 31 / 1995$ & $6 / 5 / 1995$ & 135.0 & Cake & -- & 100 & 2,308 & 391 & 2.90 & 72 & Wheat & -- \\
\hline 327 & 6/7/1995 & 7/3/1995 & 195.0 & Cake & -- & 134 & 3,039 & 547 & 2.81 & 69 & Wheat & -- \\
\hline 317 & 6/8/1995 & $6 / 12 / 1995$ & 70.0 & Cake & -- & 50 & 1,149 & 203 & 2.90 & 68 & Wheat & -- \\
\hline 326 & $6 / 10 / 1995$ & $6 / 27 / 1995$ & 235.0 & Cake & -- & 168 & 3,773 & 671 & 2.86 & 71 & Wheat & -- \\
\hline 330 & $6 / 28 / 1995$ & $7 / 3 / 1995$ & 25.0 & Cake & -- & 33 & 735 & 134 & 5.36 & 130 & Wheat & -- \\
\hline 329 & $7 / 2 / 1995$ & $7 / 13 / 1995$ & 50.0 & Cake & -- & 58 & 1,330 & 241 & 4.82 & 110 & Wheat & -- \\
\hline 332 & $7 / 3 / 1995$ & $7 / 19 / 1995$ & 160.0 & Cake & -- & 229 & 5,184 & 940 & 5.88 & 137 & Wheat & -- \\
\hline
\end{tabular}


Table 1. Biosolids applications to the study area near Deer Trail, Colorado, 1993 through 1999._-Continued

[All information provided by the Metro Wastewater Reclamation District; DC, destination code; wT, wet tons; dT, dry tons; Cake, Table 3 Class B biosolids; --, no information available; lb, pound; MAC, biosolids ammended with wood fiber; incorporated, biosolids are pushed down into the soil 4 to 8 inches after application by the tines of a rotating implement pulled by a tractor]

\begin{tabular}{|c|c|c|c|c|c|c|c|c|c|c|c|c|}
\hline $\begin{array}{c}\text { DC } \\
\text { (fig. 5) }\end{array}$ & $\begin{array}{l}\text { Application } \\
\text { start date }\end{array}$ & $\begin{array}{l}\text { Application } \\
\text { stop date }\end{array}$ & $\begin{array}{c}\text { Area } \\
\text { applied, } \\
\text { acre }\end{array}$ & $\begin{array}{l}\text { Product } \\
\text { applied }\end{array}$ & $\begin{array}{c}\text { Application } \\
\text { method }\end{array}$ & $\begin{array}{l}\text { Number } \\
\text { of loads } \\
\text { applied }\end{array}$ & $\begin{array}{c}\text { Total } \\
\text { biosolids } \\
\text { applied, } \\
\text { wT }\end{array}$ & $\begin{array}{l}\text { Total } \\
\text { biosolids } \\
\text { applied, } \\
\text { dT }\end{array}$ & $\begin{array}{l}\text { Biosolids } \\
\text { loading rate, } \\
\text { dT/acre }\end{array}$ & $\begin{array}{l}\text { Nitrogen } \\
\text { loading rate, } \\
\text { lb/acre }\end{array}$ & Crop & $\begin{array}{c}\text { Reclamation } \\
\text { project }^{1}\end{array}$ \\
\hline 328 & 7/20/1995 & $8 / 13 / 1995$ & 309.1 & Cake & -- & 455 & 10,010 & 1,762 & 5.70 & 140 & Wheat & -- \\
\hline 306 & $4 / 24 / 1997$ & 7/31/1997 & 232.0 & Cake & -- & 153 & 3,501 & 612 & 2.64 & 58 & Wheat & -- \\
\hline 307 & 6/10/1997 & 7/30/1997 & 250.0 & Cake & -- & 135 & 3,127 & 533 & 2.13 & 48 & Wheat & -- \\
\hline 314 & $6 / 17 / 1997$ & 7/1/1997 & 92.0 & Cake & -- & 67 & 1,532 & 263 & 2.86 & 63 & Wheat & -- \\
\hline 312 & 7/1/1997 & 7/17/1997 & 185.3 & Cake & -- & 134 & 3,061 & 524 & 2.83 & 69 & Wheat & -- \\
\hline 313 & 7/7/1997 & 7/17/1997 & 179.0 & Cake & -- & 128 & 2,878 & 482 & 2.69 & 61 & Wheat & -- \\
\hline 316 & 7/11/1997 & 7/13/1997 & 95.0 & Cake & -- & 66 & 1,513 & 241 & 2.54 & 52 & Wheat & -- \\
\hline 317 & 7/13/1997 & $7 / 22 / 1997$ & 155.0 & Cake & -- & 113 & 2,616 & 433 & 2.79 & 67 & Wheat & -- \\
\hline 315 & 7/18/1997 & 7/18/1997 & 19.0 & Cake & -- & 7 & 159 & 28 & 1.47 & 30 & Wheat & -- \\
\hline 319 & $7 / 22 / 1997$ & 7/23/1997 & 66.0 & Cake & -- & 41 & 917 & 154 & 2.33 & 64 & Wheat & -- \\
\hline 318 & 7/24/1997 & 7/27/1997 & 124.0 & Cake & -- & 67 & 1,432 & 242 & 1.95 & 49 & Wheat & -- \\
\hline 309 & 8/9/1997 & $1 / 29 / 1998$ & 320.0 & Cake & -- & 123 & 2,779 & 476 & 1.49 & 38 & Wheat & -- \\
\hline 308 & $8 / 24 / 1997$ & $9 / 7 / 1997$ & 314.0 & Cake & -- & 212 & 4,817 & 827 & 2.63 & 70 & Wheat & -- \\
\hline 310 & 9/7/1997 & 10/5/1997 & 299.1 & Cake & -- & 177 & 4,039 & 681 & 2.28 & 68 & Wheat & -- \\
\hline 311 & 9/13/1997 & 10/1/1997 & 320.0 & Cake & -- & 157 & 3,600 & 599 & 1.87 & 50 & Wheat & -- \\
\hline 303 & 9/17/1997 & 9/20/1997 & 301.6 & Cake & -- & 78 & 1,752 & 298 & 0.99 & 25 & Wheat & -- \\
\hline 305 & $10 / 5 / 1997$ & 2/14/1998 & 270.0 & Cake & -- & 105 & 2,283 & 370 & 1.37 & 39 & Wheat & -- \\
\hline 304 & 10/6/1997 & 10/11/1997 & 267.4 & Cake & -- & 112 & 2,447 & 405 & 1.51 & 41 & Wheat & -- \\
\hline 302 & 10/13/1997 & $1 / 29 / 1998$ & 301.6 & Cake & -- & 116 & 2,550 & 416 & 1.38 & 34 & Wheat & -- \\
\hline 301 & $1 / 29 / 1998$ & 2/4/1998 & 286.2 & Cake & -- & 111 & 2,501 & 410 & 1.43 & 39 & Wheat & -- \\
\hline 300 & 2/4/1998 & 2/9/1998 & 286.2 & Cake & -- & 108 & 2,432 & 386 & 1.35 & 38 & Wheat & -- \\
\hline 318 & 4/16/1999 & $6 / 30 / 1999$ & 44.3 & Cake/MAC & Incorporated & 98 & 2,148 & 370 & 7.18 & 209 & Oats, grass & Yes \\
\hline 316 & 4/27/1999 & 6/9/1999 & 153.0 & Cake/MAC & Incorporated & 207 & 4,573 & 799 & 5.22 & 133 & Oats, grass & Yes \\
\hline 317 & 6/9/1999 & $3 / 4 / 2000$ & 159.6 & Cake/MAC & Incorporated & 722 & 16,122 & 2,674 & 16.30 & 415 & Oats, grass & Yes \\
\hline 326 & 6/29/1999 & 11/27/1999 & 145.1 & Cake/MAC & Incorporated & 600 & 12,393 & 2,211 & 14.63 & 375 & Oats, grass & Yes \\
\hline 319 & 7/9/1999 & 12/16/1999 & 94.3 & Cake/MAC & Incorporated & 115 & 2,600 & 441 & 4.37 & 106 & Oats, grass & Yes \\
\hline 319 & 7/9/1999 & $2 / 28 / 2000$ & 94.3 & Cake/MAC & Incorporated & 137 & 3,094 & 519 & 5.20 & 130 & Oats, grass & Yes \\
\hline 327 & 7/30/1999 & 10/26/1999 & 125.5 & Cake/MAC & Incorporated & 496 & 11,073 & 1,773 & 14.00 & 371 & Oats, grass & Yes \\
\hline 325 & 10/31/1999 & 12/9/1999 & 74.0 & Cake/MAC & Incorporated & 284 & 6,266 & 959 & 12.53 & 355 & Oats, grass & Yes \\
\hline 324 & 11/7/1999 & $12 / 25 / 1999$ & 77.3 & Cake/MAC & Incorporated & 311 & 6,962 & 1,069 & 13.45 & 364 & Oats, grass & Yes \\
\hline
\end{tabular}

${ }^{1}$ As described by Colorado Department of Public Health and Environment (1998). 


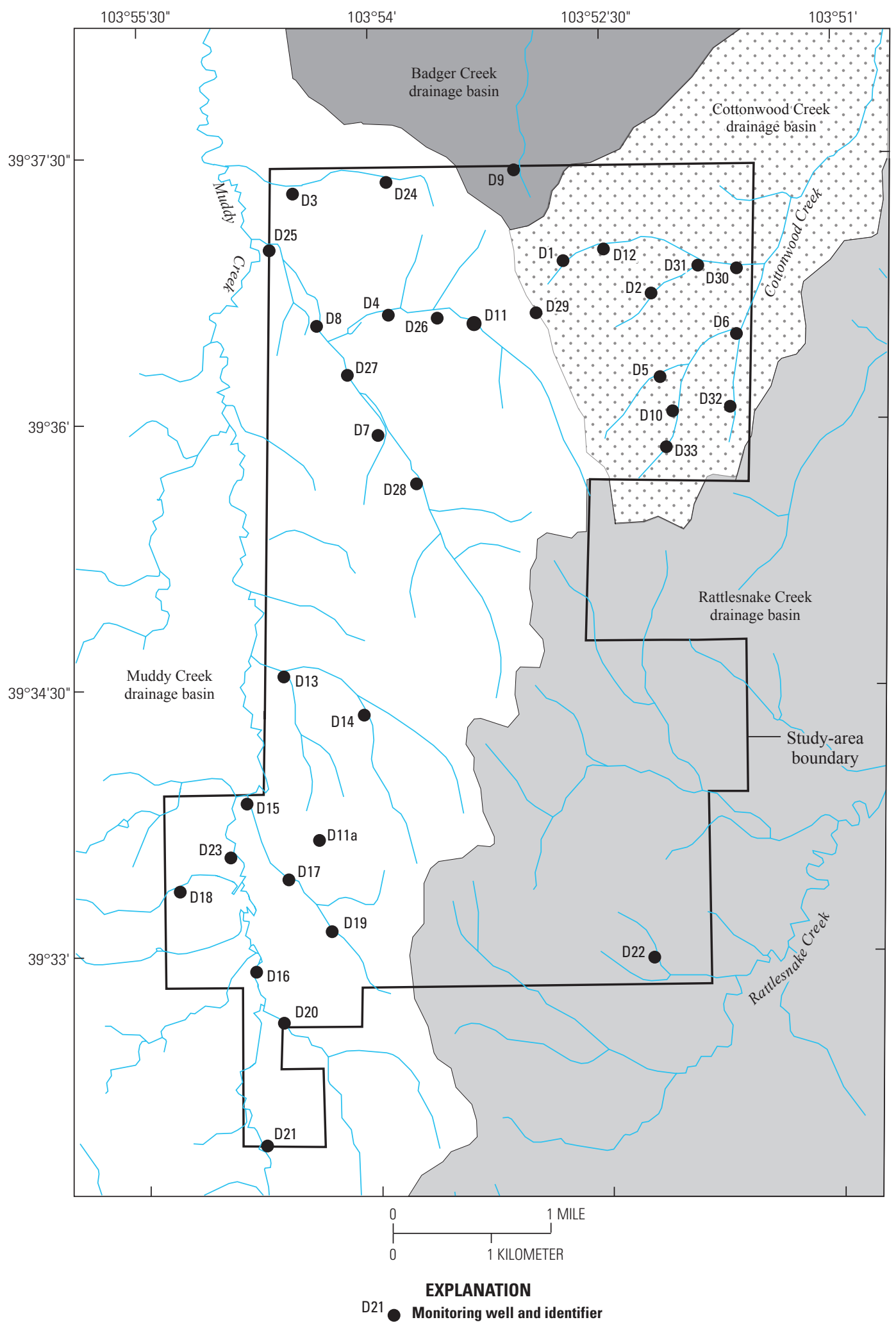

Figure 6. Drainage basins in the study area. 


\section{Approach}

To evaluate the water quality of the study area, the USGS used multiple approaches. Water-quality samples were collected through baseline sampling, reconnaissance sampling, routine sampling, and specialty sampling. Additional water-quality data were collected through other specialty monitoring, such as the use of in-situ instruments to continuously record water-quality data. Core samples also were collected and analyzed. The resulting data were compared to evaluate temporal and spatial (geographic) differences. Water-quality data also were compared to regulatory standards to evaluate the magnitude of concentrations. Limited geochemical modeling was done to evaluate flow paths and the evolution of groundwater in a selected part of the study area.

Baseline sampling was done in 1993 to provide information about water quality in the study area before biosolids applications began. To qualify as a baseline-sampling site, the site needed to be identified and sampled at least twice before biosolids were applied, and the site needed to be accessible and consistently have sufficient water for sampling throughout the 1993-1998 study period. Unfortunately, not all parts of the study area were accessible because of farming and there was insufficient time to install all the planned monitoring wells before biosolids applications started in the study area, so only some of the planned monitoring wells could be used as baselinesampling sites. The USGS installed 11 new wells in the study area (wells D1 through D11) in 1993, however, no baseline samples could be collected from two of these wells (D1 and D11) because they never contained enough water to sample even though they were located in drainage valleys that seemed likely to contain alluvial aquifers (table 2). The USGS and the Metro District removed pump equipment from an old livestockwatering well (D12) in 1993 and included that well in the baseline sampling. All baseline samples were collected by the USGS before any biosolids were applied to the study area. Baseline samples were analyzed by the Metro District laboratory for selected major ions, nutrients, and trace elements in filtered samples and bacteria (fecal coliform and fecal streptococcus bacteria) in unfiltered samples. Baseline sampling at each site usually was completed within 1 month.

Reconnaissance sampling was done during 1993 through 1995 to establish the spatial variability in water quality of the study area. The reconnaissance-sampling data also provided information that was used to determine the most suitable sampling methods and the number and location of routine waterquality sampling sites. In addition to the water-quality samples collected from the 10 monitoring wells installed in 1993 (wells D2 through D10 and D12), water-quality grab samples were collected from windmill-pumped livestock wells in the study area in 1993. The resulting chemical data indicated that water samples from the windmill-pumped livestock wells were affected by the extraction mechanisms and not representative of natural groundwater so sampling at the windmill-pumped livestock wells was discontinued. Additional shallow monitoring wells (wells D13 through D28 and wells D30 through D33) were installed and sampled during 1994 and 1995, and groundwater from wells D2 through D10 and D12 was sampled again for comparison. During reconnaissance sampling in 1994 and 1995, each monitoring well was sampled at least annually. Water-quality grab samples also were collected from streams and ponds in drainage valleys in the study area, but the transient nature of these surface-water features made them challenging for baseline or routine sampling because subsequent samples could not be collected or were not comparable. Reconnaissance samples were analyzed by the Metro District laboratory for selected major ions, nutrients, and trace elements in filtered samples, and bacteria in unfiltered samples. Based on previous studies of the effect of sewage disposal on groundwater quality in Colorado (Robson, 1977; Gaggiani, 1991; Johncox and Gaggiani, 1991; Tindall and others, 1994; and Lull and Gaggiani, 1996), biosolids effects were expected to be apparent from inorganic and bacteriological water-quality data that were compared to baseline or control-site data.

Routine sampling was done during 1995 through 1998 to establish the temporal variation in water quality after biosolids applications began. The data resulting from baseline and reconnaissance sampling indicated spatial and temporal variability in water quality, so a better understanding of groundwater quality of the study area required sampling a variety of well locations throughout the study area and repetitive sampling of the same sites at different times of the year. Therefore, inorganic waterquality samples were collected quarterly from the monitoring wells. Routine sampling focused on groundwater sites rather than surface-water sites because of the ephemeral nature of the streams and the flashy, unpredictable, powerful flows through the stream valleys in that area. Crest-stage gages installed in the study area by the USGS in 1994 were washed away during the first summer of operation so autosamplers were not deployed to sample surface water. Inorganic samples from routine sampling were analyzed for selected physical properties, major ions, nutrients, and trace elements in filtered samples at the Metro District laboratory. Bacteria samples from routine sampling were collected twice each year from the monitoring wells and occasionally from nearby ponds. The bacteria samples were analyzed for fecal coliform and fecal streptococcus bacteria at the Metro District laboratory.

Specialty sampling for unsaturated-zone pore water was initiated by the USGS in 1994 to determine if pore-water chemistry under biosolids-applied farmland differed from pore-water chemistry under farmland that was not applied with biosolids. Four unsaturated-zone plots were prepared in the study area (fig. 2), including a control plot (V4) where biosolids were not applied. Each plot was instrumented with nine suction lysimeters (Tindall and Kunkel, 1999) (fig. 2) to extract the pore water. Only some of the 36 lysimeters yielded pore-water samples in April 1994; no lysimeters in the control plot yielded pore water. No lysimeters in any of the unsaturated-zone plots yielded pore-water samples in May 1995 despite the wet spring weather that preceded the sampling attempts. Therefore, the pore-water sampling at the unsaturated-zone plots was discontinued in 1996. Pore-water samples were analyzed for selected major ions, nutrients, and trace elements in filtered samples at the Metro District laboratory. 
[Details of monitoring-well location and completion are listed in appendix 1; B, baseline water-quality compared with post-application water quality; F, water-quality flow path information; V, aquifer spatial variability; U, USGS-installed monitoring well; Metro District, Metro Wastewater Reclamation District; d, Continuous-recorder instrumentation; P, water quality at property boundary; s, interactions between surface water and groundwater; A, shallow-deep aquifer interactions; e, old livestock well with windmill or other pump; K, owned by Metro Wastewater Reclamation District until 1995 then transferred to a private owner; C, control site with no biosolids applied; c, core-sample chemistry; r, reconnaissance sampling only; t, water trough; n, none; Z, unsaturated-zone pore-water chemistry; L, suction lysimeters at depths of $1.5,3$, and 5 feet]

\begin{tabular}{|c|c|c|c|c|c|c|c|c|c|c|}
\hline $\begin{array}{c}\text { Site } \\
\text { (fig. 2) }\end{array}$ & $\begin{array}{l}\text { Sample } \\
\text { type }\end{array}$ & $\begin{array}{l}\text { Sampling } \\
\text { purpose }\end{array}$ & $\begin{array}{c}\text { Target } \\
\text { sampling } \\
\text { frequency }\end{array}$ & $\begin{array}{l}\text { Site } \\
\text { type }\end{array}$ & $\begin{array}{c}\text { Site } \\
\text { installation }\end{array}$ & $\begin{array}{l}\text { Topographic } \\
\text { setting }\end{array}$ & $\begin{array}{c}\text { Drainage } \\
\text { basin } \\
\text { (fig. } 6 \text { ) }\end{array}$ & $\begin{array}{l}\text { Property } \\
\text { owner }\end{array}$ & County & Location $^{3}$ \\
\hline D1 & Groundwater & $\mathrm{B}, \mathrm{F}, \mathrm{V}$ & $\begin{array}{c}\text { Dry well, } \\
\text { no samples available }\end{array}$ & Shallow well & $\mathrm{U}$ & Upland draw & $\begin{array}{l}\text { Cottonwood } \\
\text { Creek }\end{array}$ & Metro District & Arapahoe & T5S R58W S16 NE,SE \\
\hline D2 & Groundwater & $\mathrm{B}, \mathrm{F}, \mathrm{V}$ & Quarterly, if access & Shallow well & $\mathrm{U}, \mathrm{d}$ & Flood plain & $\begin{array}{l}\text { Cottonwood } \\
\text { Creek }\end{array}$ & Metro District & Arapahoe & T5S R58W S15 SE,SW \\
\hline D3 & Groundwater & $\mathrm{B}, \mathrm{F}, \mathrm{P}, \mathrm{V}$ & Quarterly, if access & Shallow well ${ }^{1}$ & $\mathrm{U}$ & Flood plain & Muddy Creek & Metro District & Arapahoe & T5S R58W S17 NW,NW \\
\hline D4 & Groundwater & $\mathrm{B}, \mathrm{F}, \mathrm{V}$ & Quarterly, if access & Shallow well ${ }^{1}$ & $\mathrm{U}$ & Flood plain & Muddy Creek & Metro District & Arapahoe & T5S R58W S17 SE,SE \\
\hline D5 & Groundwater & $\mathrm{B}, \mathrm{F}, \mathrm{s}, \mathrm{V}$ & Quarterly, if access & Shallow well & $\mathrm{U}$ & Flood plain & $\begin{array}{l}\text { Cottonwood } \\
\text { Creek }\end{array}$ & Metro District & Arapahoe & T5S R58W S22 SE,NW \\
\hline D6 & Groundwater & $\mathrm{B}, \mathrm{F}, \mathrm{P}, \mathrm{V}$ & Quarterly, if access & $\begin{array}{l}\text { Alluvial- } \\
\text { aquifer well }\end{array}$ & $\mathrm{U}, \mathrm{d}$ & Flood plain & $\begin{array}{l}\text { Cottonwood } \\
\text { Creek }\end{array}$ & Metro District & Arapahoe & T5S R58W S22 NE,NE \\
\hline D7 & Groundwater & $\mathrm{B}, \mathrm{F}, \mathrm{V}$ & Quarterly, if access & Shallow well ${ }^{2}$ & $\mathrm{U}$ & Flood plain & Muddy Creek & Metro District & Arapahoe & T5S R58W S20 NW,SE \\
\hline D8 & Groundwater & $\mathrm{B}, \mathrm{F}, \mathrm{V}$ & Quarterly, if access & Shallow well ${ }^{2}$ & $\mathrm{U}$ & Flood plain & Muddy Creek & Metro District & Arapahoe & T5S R58W S17 SE,SW \\
\hline D9 & Groundwater & $\mathrm{B}, \mathrm{F}, \mathrm{P}, \mathrm{V}$ & Quarterly, if access & $\begin{array}{l}\text { Bedrock- } \\
\text { aquifer well }\end{array}$ & $\mathrm{U}$ & Upland draw & Badger Creek & Metro District & Arapahoe & T5S R58W S16 NW,NE \\
\hline D10 & Groundwater & $\mathrm{B}, \mathrm{F}, \mathrm{V}$ & Quarterly, if access & Shallow well & U & Stream channel & $\begin{array}{l}\text { Cottonwood } \\
\text { Creek }\end{array}$ & Metro District & Arapahoe & T5S R58W S22 NW,SE \\
\hline D11 & Groundwater & $\mathrm{B}, \mathrm{F}, \mathrm{V}$ & $\begin{array}{c}\text { Dry well, } \\
\text { no samples available }\end{array}$ & Shallow well & $\mathrm{U}$ & Flood plain & Muddy Creek & Metro District & Arapahoe & T5S R58W S16 SE,SW \\
\hline D11a & Groundwater & $\mathrm{A}, \mathrm{F}, \mathrm{V}$ & Quarterly, if access & $\begin{array}{l}\text { Bedrock- } \\
\text { aquifer well }\end{array}$ & $\mathrm{U}$ & Hilltop & Muddy Creek & Metro District & Arapahoe & T6S R58W S5 NE,NW \\
\hline D12 & Groundwater & $\mathrm{B}, \mathrm{F}, \mathrm{V}$ & Quarterly, if access & Shallow well ${ }^{1}$ & e & Flood plain & $\begin{array}{l}\text { Cottonwood } \\
\text { Creek }\end{array}$ & Metro District & Arapahoe & T5S R58W S15 SE,NW \\
\hline D13 & Groundwater & $\mathrm{F}, \mathrm{P}, \mathrm{V}$ & Quarterly, if access & Shallow well & U & Flood plain & Muddy Creek & Metro District & Arapahoe & T5S R58W S32 NW,NW \\
\hline D14 & Groundwater & $\mathrm{F}, \mathrm{V}$ & Quarterly, if access & Shallow well & $\mathrm{U}$ & Flood plain & Muddy Creek & Metro District & Arapahoe & T5S R58W S32 SW,NE \\
\hline D15 & Groundwater & $\mathrm{F}, \mathrm{P}, \mathrm{s}, \mathrm{V}$ & Quarterly, if access & Shallow well ${ }^{2}$ & $\mathrm{U}$ & Flood plain & Muddy Creek & $\mathrm{K}$ & Elbert & T6S R58W S5 NW,NW \\
\hline D16 & Groundwater & $\mathrm{F}, \mathrm{s}, \mathrm{V}$ & Quarterly, if access & Shallow well & $\mathrm{U}$ & Flood plain & Muddy Creek & $\mathrm{K}$ & Elbert & T6S R58W S6 SE,SE \\
\hline D17 & Groundwater & $\mathrm{F}, \mathrm{V}$ & Quarterly, if access & $\begin{array}{l}\text { Alluvial- } \\
\text { aquifer well }\end{array}$ & $\mathrm{U}$ & Flood plain & Muddy Creek & Metro District & Elbert & T6S R58W S5 SE,NW \\
\hline D18 & Groundwater & $\mathrm{F}, \mathrm{P}, \mathrm{V}$ & $\begin{array}{c}\text { Dry well, } \\
\text { no samples available }\end{array}$ & Shallow well & $\mathrm{U}$ & Stream channel & Muddy Creek & $\mathrm{K}$ & Elbert & T6S R58W S6 SW,NE \\
\hline D19 & Groundwater & $\mathrm{F}, \mathrm{V}$ & Quarterly, if access & Shallow well ${ }^{1}$ & $\mathrm{U}$ & Upland draw & Muddy Creek & Metro District & Elbert & T6S R58W S5 NW,SE \\
\hline D20 & Groundwater & F, V & Quarterly, if access & Shallow well & $\mathrm{U}$ & Flood plain & Muddy Creek & K & Elbert & T6S R58W S8 NW,NW \\
\hline D21 & Groundwater & $\mathrm{C}, \mathrm{F}, \mathrm{P}, \mathrm{V}$ & Quarterly, if access & Shallow well & $\mathrm{U}$ & Flood plain & Muddy Creek & $\mathrm{K}$ & Elbert & T6S R58W S8 SW,SW \\
\hline D22 & Groundwater & F, P, V & Quarterly, if access & Shallow well ${ }^{1}$ & $\mathrm{U}$ & Flood plain & $\begin{array}{l}\text { Rattlesnake } \\
\text { Creek }\end{array}$ & Metro District & Elbert & T6S R58W S3 SW,SE \\
\hline D23 & Groundwater & $\mathrm{F}, \mathrm{s}, \mathrm{V}$ & Quarterly, if access & Shallow well ${ }^{2}$ & $\mathrm{U}, \mathrm{d}$ & Flood plain & Muddy Creek & $\mathrm{K}$ & Elbert & T6S R58W S6 NE,NE \\
\hline D24 & Groundwater & $\mathrm{A}, \mathrm{F}, \mathrm{s}, \mathrm{V}$ & Quarterly, if access & Shallow well ${ }^{1}$ & $\mathrm{U}$ & Stream channel & Muddy Creek & Metro District & Arapahoe & T5S R58W S17 NW,NE \\
\hline
\end{tabular}


Table 2. U.S. Geological Survey sampling sites near Deer Trail, Colorado, 1993 through 1999._Continued

[Details of monitoring-well location and completion are listed in appendix 1; B, baseline water-quality compared with post-application water quality; F, water-quality flow path information; V, aquifer spatial variability; U, USGS-installed monitoring well; Metro District, Metro Wastewater Reclamation District; d, Continuous-recorder instrumentation; P, water quality at property boundary; s, interactions between surface water and groundwater; A, shallow-deep aquifer interactions; e, old livestock well with windmill or other pump; K, owned by Metro Wastewater Reclamation District until 1995 then transferred to a private owner; C, control site with no biosolids applied; c, core-sample chemistry; r, reconnaissance sampling only; t, water trough; n, none; Z, unsaturated-zone pore-water chemistry; L, suction lysimeters at depths of $1.5,3$, and 5 feet]

\begin{tabular}{|c|c|c|c|c|c|c|c|c|c|c|}
\hline $\begin{array}{l}\text { Site } \\
\text { (fig. 2) }\end{array}$ & $\begin{array}{l}\text { Sample } \\
\text { type }\end{array}$ & $\begin{array}{l}\text { Sampling } \\
\text { purpose }\end{array}$ & $\begin{array}{c}\text { Target } \\
\text { sampling } \\
\text { frequency }\end{array}$ & $\begin{array}{l}\text { Site } \\
\text { type }\end{array}$ & $\begin{array}{c}\text { Site } \\
\text { installation }\end{array}$ & $\begin{array}{l}\text { Topographic } \\
\text { setting }\end{array}$ & $\begin{array}{l}\text { Drainage } \\
\text { basin } \\
\text { (fig. 6) }\end{array}$ & $\begin{array}{l}\text { Property } \\
\text { owner }\end{array}$ & County & Location $^{3}$ \\
\hline $\mathrm{D} 25$ & Groundwater & $\mathrm{F}, \mathrm{P}, \mathrm{s}, \mathrm{V}$ & Quarterly, if access & $\begin{array}{c}\text { Alluvial- } \\
\text { aquifer well }\end{array}$ & $\mathrm{U}$ & Flood plain & Muddy Creek & Metro District & Arapahoe & T5S R58W S17 SW,NW \\
\hline D26 & Groundwater & $\mathrm{F}, \mathrm{V}$ & Quarterly, if access & Shallow well ${ }^{1}$ & $\mathrm{U}$ & Flood plain & Muddy Creek & Metro District & Arapahoe & T5S R58W S16 SW,SW \\
\hline D27 & Groundwater & $\mathrm{F}, \mathrm{V}$ & Quarterly, if access & Shallow well ${ }^{2}$ & $\mathrm{U}$ & Flood plain & Muddy Creek & Metro District & Arapahoe & T5S R58W S20 SW,NE \\
\hline D28 & Groundwater & $\mathrm{F}, \mathrm{V}$ & Quarterly, if access & Shallow well ${ }^{2}$ & U & Stream channel & Muddy Creek & Metro District & Arapahoe & T5S R58W S20 SE,SE \\
\hline D29 & Groundwater & $\mathrm{A}, \mathrm{F}, \mathrm{V}$ & Quarterly, if access & $\begin{array}{l}\text { Bedrock- } \\
\text { aquifer well }\end{array}$ & $\mathrm{U}$ & Hilltop & $\begin{array}{l}\text { Cottonwood } \\
\text { Creek }\end{array}$ & Metro District & Arapahoe & T5S R58W S16 SW,SW \\
\hline D30 & Groundwater & $\mathrm{F}, \mathrm{P}, \mathrm{s}, \mathrm{V}$ & Quarterly, if access & Shallow well ${ }^{2}$ & $\mathrm{U}$ & Stream channel & $\begin{array}{l}\text { Cottonwood } \\
\text { Creek }\end{array}$ & Metro District & Arapahoe & T5S R58W S15 NE,SE \\
\hline D31 & Groundwater & $\mathrm{F}, \mathrm{V}, \mathrm{c}$ & Quarterly, if access & $\begin{array}{l}\text { Alluvial- } \\
\text { aquifer well }\end{array}$ & $\mathrm{U}$ & Flood plain & $\begin{array}{l}\text { Cottonwood } \\
\text { Creek }\end{array}$ & Metro District & Arapahoe & T5S R58W S15 NW,SE \\
\hline D32 & Groundwater & $\mathrm{F}, \mathrm{V}$ & Quarterly, if access & Shallow well ${ }^{1}$ & $\mathrm{U}$ & Stream channel & $\begin{array}{l}\text { Cottonwood } \\
\text { Creek }\end{array}$ & Metro District & Arapahoe & T5S R58W S15 SE,NE \\
\hline D33 & Groundwater & $\mathrm{F}, \mathrm{V}, \mathrm{c}$ & Quarterly, if access & $\begin{array}{l}\text { Alluvial- } \\
\text { aquifer well }\end{array}$ & $\mathrm{U}$ & Flood plain & $\begin{array}{l}\text { Cottonwood } \\
\text { Creek }\end{array}$ & Metro District & Arapahoe & T5S R58W S22 NW,SE \\
\hline G1 & Groundwater & $\mathrm{r}$ & Occasionally & $\begin{array}{c}\text { Trough of } \\
\text { livestock well }\end{array}$ & $\mathrm{e}, \mathrm{t}$ & Flood plain & $\begin{array}{l}\text { Rattlesnake } \\
\text { Creek }\end{array}$ & Metro District & Elbert & T6S R58W S4 SW1/4 SE1/4 \\
\hline G2 & Groundwater & $\mathrm{r}$ & Occasionally & $\begin{array}{c}\text { Trough of } \\
\text { livestock well }\end{array}$ & $\mathrm{e}, \mathrm{t}$ & Flood plain & Muddy Creek & K & Elbert & T6S R58W S8 NW1/4 SE1/4 \\
\hline G3 & Groundwater & $\mathrm{r}$ & Occasionally & $\begin{array}{c}\text { Trough of } \\
\text { livestock well }\end{array}$ & $\mathrm{e}, \mathrm{t}$ & Flood plain & Muddy Creek & Metro District & Arapahoe & T5S R58W S20 SW1/4 SW1/4 \\
\hline S1 & Surface water & $\mathrm{r}, \mathrm{s}$ & Occasionally & Stream & $\mathrm{n}$ & Stream channel & $\begin{array}{l}\text { Rattlesnake } \\
\text { Creek }\end{array}$ & Metro District & Elbert & T6S R58W S3 SE1/4 SE1/4 \\
\hline S2 & Surface water & $\mathrm{r}, \mathrm{s}$ & Occasionally & Near shore & $\mathrm{n}$ & Lake or swamp & $\begin{array}{c}\text { Rattlesnake } \\
\text { Creek }\end{array}$ & Metro District & Elbert & T6S R58W S3 SE1/4 SE1/4 \\
\hline S3 & Surface water & $\mathrm{r}, \mathrm{s}$ & Occasionally & Near east shore & $\mathrm{n}$ & $\begin{array}{c}\text { Ponded } \\
\text { stream channel }\end{array}$ & Muddy Creek & K & Elbert & T6S R58W S8 NW1/4 SW1/4 \\
\hline S4 & Surface water & $\mathrm{r}, \mathrm{s}$ & Occasionally & $\begin{array}{c}\text { Near shore } \\
\text { by well D30 }\end{array}$ & $\mathrm{n}$ & $\begin{array}{c}\text { Ponded } \\
\text { stream channel }\end{array}$ & $\begin{array}{l}\text { Cottonwood } \\
\text { Creek }\end{array}$ & Metro District & Arapahoe & T5S R58W S15 NE,SE \\
\hline S5 & Surface water & $\mathrm{r}, \mathrm{s}$ & Occasionally & $\begin{array}{l}\text { Small swamp } \\
\text { near well D15 }\end{array}$ & $\mathrm{n}$ & Lake or swamp & Muddy Creek & $\mathrm{K}$ & Elbert & T6S R58W S5 NW,NW \\
\hline S6 & Surface water & $\mathrm{r}, \mathrm{s}$ & Occasionally & $\begin{array}{c}\text { East shore } \\
\text { near well D20 }\end{array}$ & $\mathrm{n}$ & $\begin{array}{c}\text { Ponded } \\
\text { stream channel }\end{array}$ & Muddy Creek & K & Elbert & T6S R58W S8 NW,NW \\
\hline S7 & Surface water & $\mathrm{r}, \mathrm{s}$ & Occasionally & $\begin{array}{c}\text { East shore } \\
\text { near well D23 }\end{array}$ & $\mathrm{n}$ & $\begin{array}{c}\text { Ponded } \\
\text { stream channel }\end{array}$ & Muddy Creek & K & Elbert & T6S R58W S6 NE,NE \\
\hline
\end{tabular}


Table 2. U.S. Geological Survey sampling sites near Deer Trail, Colorado, 1993 through 1999._Continued

[Details of monitoring-well location and completion are listed in appendix 1; B, baseline water-quality compared with post-application water quality; F, water-quality flow path information; V, aquifer spatial variability; U, USGS-installed monitoring well; Metro District, Metro Wastewater Reclamation District; d, Continuous-recorder instrumentation; P, water quality at property boundary; s, interactions between surface water and groundwater; A, shallow-deep aquifer interactions; e, old livestock well with windmill or other pump; K, owned by Metro Wastewater Reclamation District until 1995 then transferred to a private owner; C, control site with no biosolids applied; c, core-sample chemistry; r, reconnaissance sampling only; t, water trough; n, none; Z, unsaturated-zone pore-water chemistry; L, suction lysimeters at depths of $1.5,3$, and 5 feet]

\begin{tabular}{|c|c|c|c|c|c|c|c|c|c|c|}
\hline $\begin{array}{c}\text { Site } \\
\text { (fig. 2) }\end{array}$ & $\begin{array}{l}\text { Sample } \\
\text { type }\end{array}$ & $\begin{array}{l}\text { Sampling } \\
\text { purpose }\end{array}$ & $\begin{array}{c}\text { Target } \\
\text { sampling } \\
\text { frequency }\end{array}$ & $\begin{array}{l}\text { Site } \\
\text { type }\end{array}$ & $\begin{array}{c}\text { Site } \\
\text { installation }\end{array}$ & $\begin{array}{l}\text { Topographic } \\
\text { setting }\end{array}$ & $\begin{array}{l}\text { Drainage } \\
\text { basin } \\
\text { (fig. 6) }\end{array}$ & $\begin{array}{l}\text { Property } \\
\text { owner }\end{array}$ & County & Location $^{3}$ \\
\hline $\mathrm{V} 1^{4}$ & $\begin{array}{l}\text { Unsaturated-zone } \\
\text { pore water }\end{array}$ & $\mathrm{F}, \mathrm{Z}$ & $\begin{array}{l}\text { Attempted annually } \\
\text { (1994 and 1995) }\end{array}$ & $\begin{array}{c}\text { Farmed plot } \\
\text { with } 9 \text { lysimeters }\end{array}$ & $\mathrm{L}$ & Hillside & $\begin{array}{l}\text { Cottonwood } \\
\text { Creek }\end{array}$ & Metro District & Arapahoe & T5S R58W S16 NE,SE \\
\hline $\mathrm{V} 2^{5}$ & $\begin{array}{l}\text { Unsaturated-zone } \\
\text { pore water }\end{array}$ & $\mathrm{F}, \mathrm{Z}$ & $\begin{array}{l}\text { Attempted annually } \\
\text { (1994 and 1995) }\end{array}$ & $\begin{array}{c}\text { Farmed plot } \\
\text { with } 9 \text { lysimeters }\end{array}$ & $\mathrm{L}$ & Hillside & $\begin{array}{l}\text { Cottonwood } \\
\text { Creek }\end{array}$ & Metro District & Arapahoe & T5S R58W S16 NE,SE \\
\hline$V 3^{6}$ & $\begin{array}{l}\text { Unsaturated-zone } \\
\text { pore water }\end{array}$ & $\mathrm{F}, \mathrm{Z}$ & $\begin{array}{l}\text { Attempted annually } \\
\text { (1994 and 1995) }\end{array}$ & $\begin{array}{c}\text { Farmed plot } \\
\text { with } 9 \text { lysimeters }\end{array}$ & $\mathrm{L}$ & Hillside & $\begin{array}{l}\text { Cottonwood } \\
\text { Creek }\end{array}$ & Metro District & Arapahoe & T5S R58W S15 SE,SW \\
\hline V4 & $\begin{array}{l}\text { Unsaturated-zone } \\
\text { pore water }\end{array}$ & C, F, Z & $\begin{array}{l}\text { Attempted annually } \\
\text { (1994 and 1995) } \\
\text { Dry lysimeters, } \\
\text { no samples available }\end{array}$ & $\begin{array}{c}\text { Farmed plot } \\
\text { with } 9 \text { lysimeters }\end{array}$ & $\mathrm{L}$ & Flood plain & Muddy Creek & Metro District & Arapahoe & T5S R58W S17 NW,NW \\
\hline
\end{tabular}

${ }^{1}$ Probably bedrock-aquifer well.

${ }^{2}$ Probably alluvial-aquifer well.

${ }^{3}$ Location indicated by township, range, section, quarter-section, and quarter of quarter section. The letters after the section number represent successive subdivisions of the section assigned directionally by quadrant (NW, northwest; NE, northeast; SE, southeast; SW, southwest).

${ }^{4}$ Samples obtained from L1 and L2, both of which were lysimeters that sampled pore water at 1.5 feet below land surface.

${ }^{5}$ Samples obtained from L3, L4, and L8, all of which were lysimeters that sampled pore water at 1.5 feet below land surface; sample also obtained from L7, a lysimeter that sampled pore water at 3 feet below land surface.

${ }^{6}$ Samples obtained from L3 and L8, both of which were lysimeters that sampled pore water at 1.5 feet below land surface. 
Specialty sampling for dissolved gases and chlorofluorocarbons (CFCs) was done at selected monitoring wells in 1998 to infer information about water quality, flow paths, and groundwater recharge. Dissolved gases that were analyzed in the groundwater samples included argon, carbon dioxide, methane, nitrogen, and oxygen. Chlorofluorocarbons are anthropogenic organic compounds in production since the 1930 's and are present in young groundwater (recharged after about 1940). Dissolved-gas and chlorofluorocarbon (DG-CFC) samples were collected one time (November 1998) at 10 monitoring wells in the study area. The DG-CFC samples were analyzed at the USGS Reston Chlorofluorocarbon Laboratory. Sampling and analytical methods for DG-CFC are described by Busenberg and Plummer (1992), Busenberg and others (1999), Plummer and Friedman (1999), Stute and others (1992), and Wilson and McNeill (1997). The resulting data were reported by Yager and Arnold (2003, tables II.5 and II.6).

Specialty sampling for oxidation-reduction (redox) information was done in 1999 to determine redox states in the groundwater. Eight monitoring wells were sampled for redox information. Redox-indicator sampling included analyses for dissolved oxygen, hydrogen gas, hydrogen sulfide, and methane in the field by the USGS. The redox-indicator samples also were analyzed for full inorganic chemistry at the USGS National Water Quality Laboratory (NWQL).

Other specialty monitoring included the use of automated data recorders during 1997 and 1998 to provide detailed information about selected water-quality characteristics. Routine water-quality sampling data provide large-scale (periodic) information about temporal and spatial water-quality variability, but continuous-recorder data provide small-scale (detailed) information about the temporal variability at a site. The large values of specific conductance indicated from baseline and reconnaissance sampling at well D6 were, therefore, examined in detail through automated data recorders. Both specific conductance and groundwater temperature were continuously recorded during 1997 and 1998. The complete data-collection methods and resulting data were reported by Yager and Arnold (2003).

Core-chemistry data from well borings in the study area also were obtained for comparison with the water-quality data. These core samples were analyzed for selected properties $(\mathrm{pH}$, specific conductance, percent organic matter, percent volatile solids, percent total solids), nutrients, and trace elements at the Metro District laboratory.

\section{Site Selection}

USGS personnel collected data from numerous sources and locations in the study area (figs. 2 and 3). Sites were selected to monitor shallow alluvial-aquifer and bedrock-aquifer groundwater, deeper bedrock-aquifer groundwater, surface water, and pore water in the unsaturated zone. Sites also were selected for continuous monitoring of groundwater-quality properties and for sampling core materials. All sites where data were collected are listed in table 2 and shown in figure 2. Additional information about site selection, such as well completion, is described by Yager and Arnold (2003). Access to the monitoring sites in the study area was challenging due to a lack of roads and the rough terrain. Farming activities, muddy conditions, large sand dunes, and large desiccation cracks in the ground occasionally prevented access to monitoring sites. These access restrictions resulted in decreased data collection during the study and created temporal gaps in the data sets.

Shallow alluvial-aquifer and bedrock-aquifer groundwater was monitored throughout the study area. Three windmill-pumped livestock wells were sampled twice in 1993 to provide approximate water-quality information for the study before wells could be installed and to help select monitoring locations. The USGS and the Metro District removed pump equipment from another livestock-watering well (D12) in 1993 and included that well in the monitoring program. Long-term monitoring wells were installed at 31 locations to monitor shallow groundwater. Shallow wells were located in the drainage valleys because the alluvial aquifers were not continuous throughout the study area but existed only in the drainage valleys or in paleochannels of flood plains. Wells were located near property boundaries to evaluate the quality of groundwater leaving the study area. Wells were located further upgradient from property boundaries to evaluate chemical and hydrologic variability within each alluvial aquifer. The first three monitoring wells (D1, D2, and D3) were located topographically downgradient from the four unsaturated-zone plots (fig. 2). The alluvial aquifers generally were located in flood plains; Yager and Arnold (2003) determined that shallow groundwater was not present at the drainage divides (the basin boundaries shown in fig. 6) where in some places biosolids were not applied. The topography (fig. 2) indicated that the biosolids-application areas in the study area were headwaters for most of the streams in the study area, so few locations were available to monitor groundwater upgradient from the biosolids-application areas. Sites that are chemically and hydrologically representative of other sampled locations in the study area but not affected by biosolids applications through infiltration, surface-water runoff, groundwater transport, or wind transport could be control sites for groundwater. Only one control site (D21) could be established for this study, although the data from well D21 samples indicated that water quality at this site was not representative of water quality at other locations in the study area.

Two deeper bedrock-aquifer wells (D11a, D29) were installed in 1997 by the USGS. These wells were located in sandstone ridges where alluvial aquifers were not present to evaluate water quality in the deeper part of the bedrock aquifer where it was not mixing with alluvial aquifers.

DG-CFC samples were collected at monitoring wells in the study area that had chemical concentrations of concern or anomalous water quality compared to other nearby wells. Ten wells were sampled for DG-CFCs: wells D3, D5, D6, D9, D10, D13, D14, D17, D24, and D25. 
Only monitoring wells where the dissolved oxygen in the groundwater was less than $1 \mathrm{mg} / \mathrm{L}$ were candidates for redoxindicator sampling. Therefore, redox information was obtained from wells D15, D16, D21, D22, D23, and D30 in the study area (fig. 2) and from wells DTX2 and DTX10 (zone A) in the USGS Expanded Monitoring Program (fig. 3) in July 1999.

Streams in the study area were ephemeral and flashy, so surface-water sampling sites consisted of the four largest ponds in the study area. These ponds all were located in drainage valleys near monitoring wells. Water-quality samples were collected occasionally from these surface-water sites to determine if the surface-water quality resembled the groundwater quality in the study area. All the surface-water sampling sites were either ephemeral or were visited by livestock so were not routinely sampled.

Four unsaturated-zone plots were selected and prepared before biosolids-applications began. The unsaturated-zone plots were located in upland areas, which were where biosolids were to be applied at the site, not drainage valleys where no biosolids could be applied (fig. 2). These unsaturated-zone plots included one control plot where biosolids were not applied (V4 near well D3), as well as three plots where biosolids were applied (V1, north of well D1; V2, south of well D1; and V3, north of well D2) (fig. 2). Each unsaturated-zone plot was about $150 \mathrm{ft}$ by $150 \mathrm{ft}$ and contained nine porouscup suction lysimeters (also called "solution samplers" by Tindall and Kunkel, 1999, p. 569). The unsaturated-zone plots were surveyed in the fall of 1993, and biosolids were applied to three of the plots (V1, V2, and V3) in December 1993. In February 1994, suction lysimeters were installed in the four unsaturated-zone plots using a portable two-person-operated auger. Each unsaturated-zone plot was instrumented with nine suction lysimeters (Tindall and Kunkel, 1999): three suction lysimeters installed at a depth of $1.5 \mathrm{ft}$, three suction lysimeters installed at a depth of $3 \mathrm{ft}$, and three suction lysimeters installed at a depth of $5 \mathrm{ft}$. The installation depth of each lysimeter was determined through a random-number table based on the method described by Lüscher (1994). The USGS attempted to sample all the suction lysimeters in each plot each pore-water-sampling trip. The unsaturated-zone plots were dismantled in 1996.

Core-sampling sites were not originally planned to be part of the study, but the initial groundwater-quality data from reconnaissance sampling indicated a likely geochemical signature in the groundwater so core sampling was added. Two of the last alluvial-aquifer boreholes drilled in 1995 (completed as wells D31 and D33) were selected as core-sampling sites. Core samples were collected from throughout the unsaturated zone of the two boreholes.

\section{Data-Collection Methods}

Data collection consisted of measuring selected waterquality properties and related parameters in the field at the time of sampling, collecting samples that were analyzed by a laboratory, and continuously recording water-quality properties in the field at selected sites. Core samples from selected sites also were collected in the field and analyzed by a laboratory. Various methods were used to collect samples and other water-quality data from the study area during 1993-1999.

\section{Field Methods Used to Collect Data for Physical Properties and Inorganic Chemical Constituents}

During baseline and reconnaissance sampling, samples were collected from the monitoring wells for inorganic analyses. Wells were purged 1-2 days in advance of sampling by handbailing or by using a submersible pump during 1993 through mid-1994. During mid-1994 through 1995, wells were purged at the time of sampling. Monitoring wells were sampled according to standard USGS methods (Sylvester and others, 1990) by using a submersible pump (most of the time) or by using a clean plastic tube with a peristaltic pump. Wells were occasionally hand bailed during 1993-1995 in cases of equipment failure. All sampling equipment was cleaned in the field between sampling sites. Standard USGS methods (Sylvester and others, 1990) were used for sample processing. The baseline and reconnaissance samples were submitted on the day of sample collection to the Metro District laboratory for analysis.

During reconnaissance sampling, grab samples were collected at an assortment of windmill-pumped livestock wells and at surface-water features in the study area for inorganic analyses. Groundwater grab samples were collected either from the discharge line of the windmill pump or, if the windmill was not pumping, from the top, center part of the water trough that was filled by the windmill-pumped well. Each groundwater grab sample was collected in a large, clean, plastic bottle then processed by filtration and sample preservation. Surface-water grab samples were collected by dipping a large, clean, plastic bottle directly into the surface-water feature and filling the bottle from along the shore near the surface. The surface-water grab samples were then processed by filtration and sample preservation. Standard USGS methods (Sylvester and others, 1990) were used for sample processing. The grab samples were submitted on the day of sample collection to the Metro District laboratory for analysis.

During routine sampling, samples were again collected from the monitoring wells for inorganic analyses. Depth to groundwater was measured before sampling procedures were started. Wells were purged until field properties stabilized (measured through a flow-through chamber) at the time of sampling using a pump. Wells that were screened deeper than $30 \mathrm{ft}$ below land surface were sampled by using a stainlesssteel submersible pump that was cleaned in the field between sites. Wells that were screened at less than $30 \mathrm{ft}$ below land surface were sampled by using a peristaltic pump with dedicated silicone and polyethylene tubing inside the well to the depth of the screened area. Samples were collected and processed during most of 1995 according to the standard USGS methods described by Sylvester and others (1990). In 1994, a national USGS research effort documented a new sampling protocol for trace-element water-quality sampling (Horowitz and others, 1994). After reviewing the data obtained through 
October 1995, the USGS decided stricter sampling and processing protocols were needed to ensure groundwater-data quality for this study because many water-quality constituents were present in such small concentrations that uncertainty contributed by field and laboratory factors made these constituents difficult to accurately quantify. Therefore, in late 1995, sampling preparations, field methods, and documentation procedures for this study were revised to follow those of Horowitz and others (1994), Koterba and others (1995), and U.S. Geological Survey (variously dated). The new sampling protocol that was used for the collection and processing of inorganic groundwater samples in the study area during late 1995 through 1999 was more appropriate for lowconcentration constituents and is summarized in figure 7. All routine water-quality samples collected during 1995 through 1998 were submitted on the day of sample collection to the Metro District laboratory for analysis.

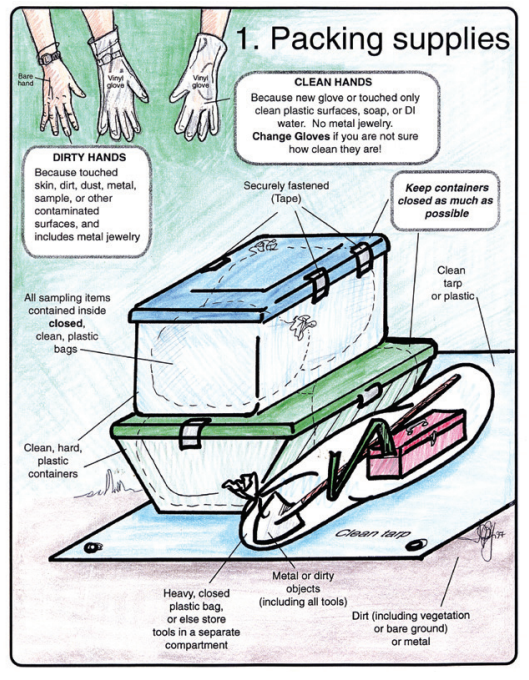

During specialty sampling for pore water, each lysimeter in all four unsaturated-zone plots was pressurized then sampled by using methods described by Tindall and Vencill (1995). Not all lysimeters yielded enough pore water for analysis; most lysimeters only yielded a little mist. The lysimeter samples that had at least $0.5 \mathrm{~mL}$ of pore water were processed at the USGS in Denver according to methods described by Tindall and Vencill (1995), which included dilution to $0.1 \mathrm{~L}$ with deionized water. The diluted pore-water samples were delivered to the Metro District laboratory for analysis.

During other specialty monitoring for specific conductance and groundwater temperature, automated data recorders were used to provide detailed data. Specific conductance and water temperature were continuously monitored at well D6 by using submersible sensors that were cabled to a data logger and deployed for several years at the same field site. These sensors were inspected during monthly site visits, and
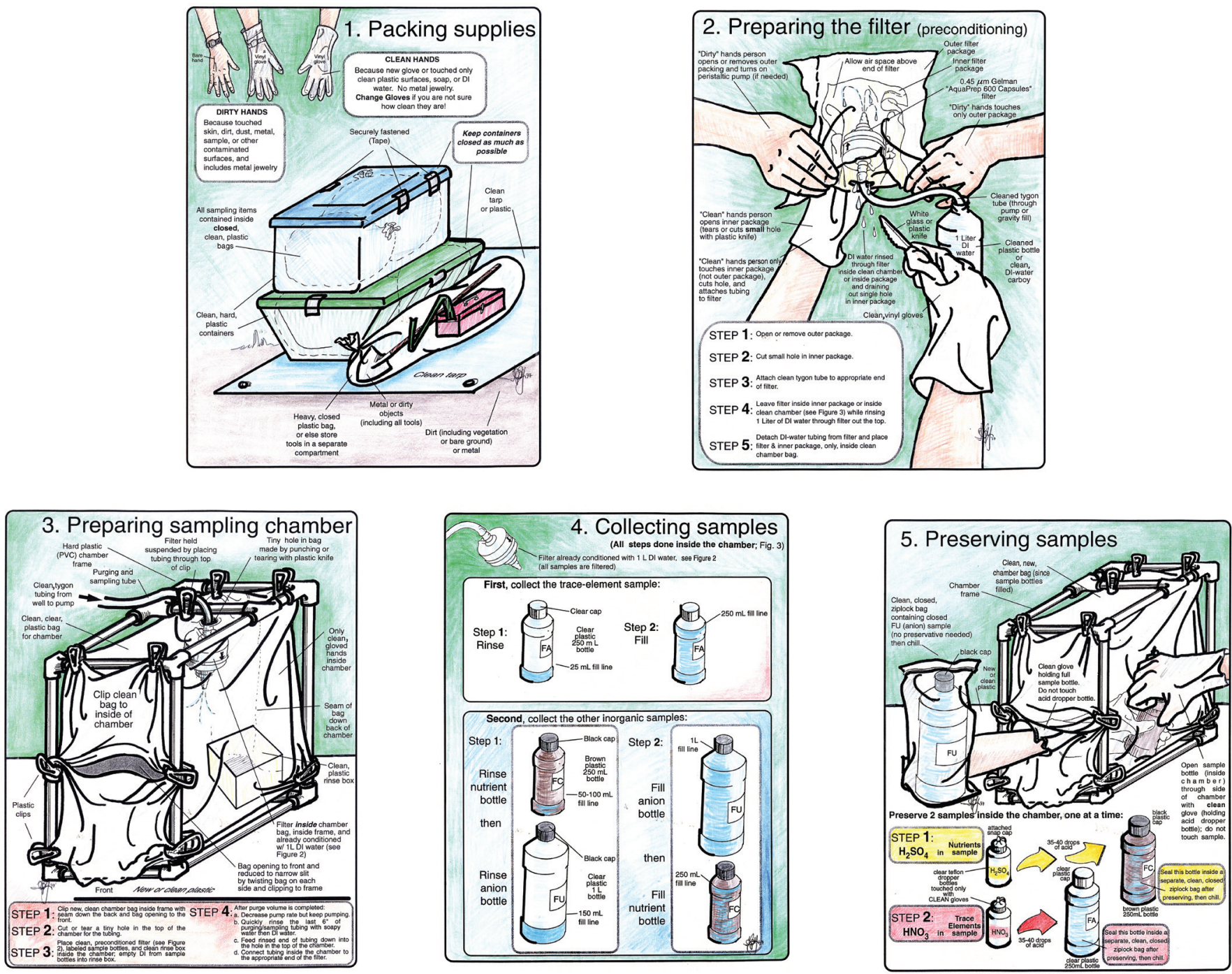

Figure 7. Summary of the U.S. Geological Survey sampling method used to collect groundwater-quality samples near Deer Trail, Colorado, late 1995 through 1999. [DI, deionized water; FA, filtered and acidified sample; FC, filtered and chilled sample; FU, filtered and unpreserved sample; L, liter; $\mathrm{PVC}$, polyvinyl chloride (plastic); $\mathrm{mL}$, milliliter; $\mathrm{H}_{2} \mathrm{SO}_{4^{\prime}}$ sulfuric acid; $\mathrm{HNO}_{3^{\prime}}$ nitric acid]

Figure 7 includes five panels. Click on the thumbnails to view the enlarged version of each panel 
data were downloaded approximately monthly from the data logger. The resulting data are reported by Yager and Arnold (2003, figure II.6). Large periods of missing record in these data were the result of flooding in the nearby drainage valley that also flooded the continuous-recorder instrumentation. Because the periods of missing record were during critical recharge periods, the monitoring period was extended to last about 2 years.

Core of shallow subsurface geologic materials was sampled when drilling was done to install two monitoring wells (D31 and D33). Core was collected from the borehole by using split-spoon core barrels in hollow-stem augers. Samples were collected from measured sections of core by using a clean plastic spoon to scrape away any part of the core that had been in contact with the core barrel and to transfer the scraped core sample to a clean sample jar that was provided by the Metro District laboratory. The core samples were delivered to the Metro District laboratory on the same day collected.

\section{Field Methods Used to Sample for Bacteriological Constituents}

Groundwater and surface-water sites in the study area were sampled for selected bacteria. All bacteria samples collected during 1993-1998 were chilled and delivered within about 4 to 6 hours of collection to the Metro District laboratory where the samples were cultured and analyzed.

From 1993 through 1996, bacteria samples were collected from unpumped wells on a different day from the inorganic water-quality sampling because of the short time period after sample collection that the samples had to be delivered to the Metro District laboratory for culturing. The sampling method utilized a single brass bailer as described by Gaggiani (1991, p. 22). The brass bailer was tied to cotton/polyester rope. Some erratic and questionable results were obtained with this method (for example, a D2 sample on 10/3/96). Therefore, sampling methods were revised in 1996 to improve the quality of bacteria data by ensuring that any contamination was not from field sampling or processing.

From 1997 through 1998, bacteria samples were collected from purged wells immediately after the inorganic groundwater samples (on the same day) using single-use autoclaved polyethylene bailers attached to bleach-washed, plastic-coated cable. The groundwater sample that was collected in the bailer was poured immediately into a clean autoclaved sample bottle that was provided by the Metro District laboratory. The revised sampling methods were described by Myers and Sylvester (1997, sections 7.1.1 and 7.1.2) and U.S. Geological Survey (variously dated).

Ponds in the study area were sampled occasionally for bacteria during 1995 through 1998. Pond samples were collected by dipping an autoclaved sample bottle that was provided by the Metro District laboratory directly into the pond for a grab sample. These surface-water grab samples were collected near the surface and bank of the pond.

\section{Field Methods Used to Sample for Other Constituents}

Specialty sampling for DG-CFCs used equipment and methods that are described by Busenberg and others (1999), Plummer and Friedman (1999), Stute and others (1992), and Wilson and McNeill (1997). A description of sampling equipment and methods, as well as the USGS applications of the resulting data, also are provided on the Internet (http://water.usgs.gov/lab/cfc and http://water.usgs.gov/lab/ dissolved-gas, accessed October 3, 2012). The DG-CFC samples were chilled and shipped immediately to Reston, Va., for analysis. Samples for inorganic constituents were not collected at this time.

Specialty sampling for redox-sensitive constituents involved a variety of field methods. Methods for sampling and processing the redox-sensitive constituents are described by Chapelle and others (1995) and Lovley and others (1994). The redox-indicator samples were delivered to a mobile field laboratory or to a USGS research laboratory in Denver for analysis. The monitoring wells that were sampled for redoxindicator constituents also were sampled for field properties, major ions, nutrients, and trace elements at the same time. The samples for these inorganic constituents were collected and processed using the same methods that were used for routine sampling during 1996 through 1998 (Horowitz and others, 1994; Koterba and others, 1995; U.S. Geological Survey, variously dated) but were delivered to the NWQL in Denver for analysis.

\section{Analytical Methods}

Various laboratories provided chemical data for samples from the study area. Both USGS laboratories and the Metro District laboratory analyzed samples during 1993-1999.

The Metro District laboratory in Denver provided all inorganic analyses of environmental samples during 1993-1998, including the analyses of groundwater, surfacewater, pore-water, and core samples. The water-quality samples of groundwater, surface water, and pore water were analyzed for selected physical properties, major ions, nutrients, and trace elements according to the methods listed in table 3. Groundwater and surface-water samples also were cultured and analyzed for fecal coliform and fecal streptococcus bacteria according to the methods listed in table 3 . The core samples were dried, sieved, and split for analysis at the Metro District laboratory and were analyzed for inorganic constituents using analytical methods similar to those listed in table 3 (M.L. Castlebury, Metro District laboratory, oral commun., October 1995). After the first 2 years of sampling, it was determined that the Metro District laboratory did not have sufficiently low reporting limits for characterizing most of the trace elements of interest at most of the monitoring wells, so selected groundwater samples collected during 1995-1998 also were analyzed at the NWQL. 
Table 3. Laboratory methods used by the Metro Wastewater Reclamation District Laboratory for analyses of water samples.

[All information provided by the Metro Wastewater Reclamation District (M.L. Castlebury, Metro Wastewater Reclamation District, written commun., June 1998); additional information about the analytical methods used is available from the Metro Wastewater Reclamation District; MRL, minimum reporting level, although dilutions for samples having high specific conductance resulted in higher MRL's for some samples; $\mathrm{CaCO}_{3}$, calcium carbonate; mg/L, milligrams per liter; --, no information; ICP, inductively coupled plasma; ${ }^{\circ} \mathrm{C}$, degrees Celsius; $\mathrm{N}$, nitrogen; ASF, automated segmented-flow spectrophotometry; P, phosphorus; $\mu \mathrm{g} / \mathrm{L}$, micrograms per liter; GFAA, graphite-furnace atomic absorption spectrometry; MS, mass spectroscopy; mL, milliliters]

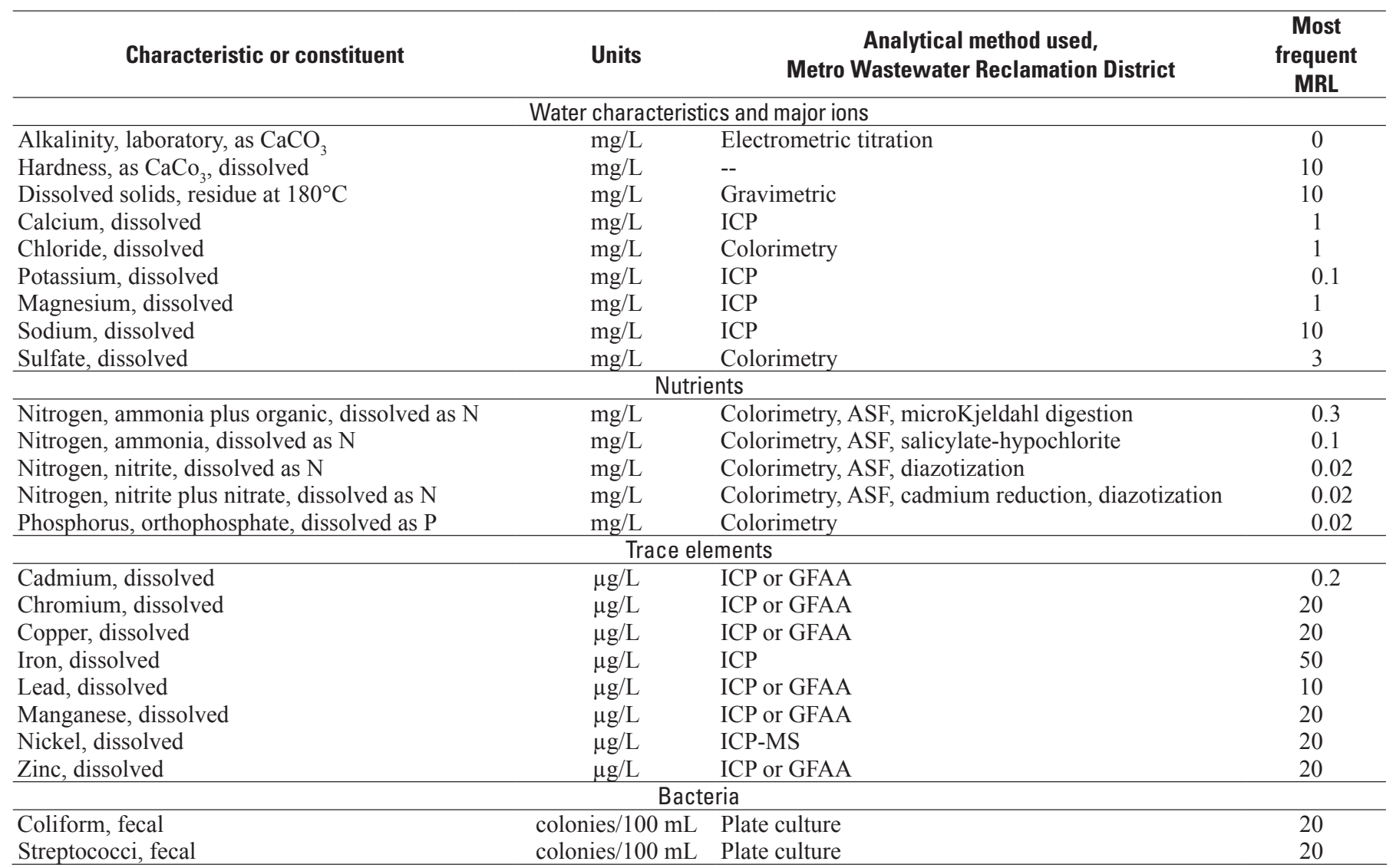

The NWQL analyzed selected groundwater-sample replicates and blanks during 1995-1998, as well as all groundwater samples collected during 1999. The analytical methods used by the NWQL are listed in table 4 . The analyses at the NWQL (table 4) included some lower minimum reporting levels (MRLs) and additional analytes (physical properties, major ions, nutrients, and trace elements) relative to the analyses at the Metro District laboratory (table 3).

The DG-CFC samples were analyzed by the USGS Chlorofluorocarbon Laboratory in Reston, Va., using analytical methods described by Busenberg and others (1999), Plummer and Friedman (1999), Stute and others (1992), and Wilson and McNeill (1997). The analytical methods also are described on the Internet at http://water.usgs.gov/lab/cfc, and http://water.usgs.gov/lab/dissolved-gas (accessed October 3, 2012).

The redox-indicator samples were analyzed in the field or analyzed by a USGS research laboratory in Denver. Methods for analyzing the redox-indicator samples are described by Chapelle and others (1995) and Lovley and others (1994).

\section{Quality-Assurance Methods}

Quality-assurance methods routinely were used by the USGS in the collection of data from the study area. These methods included replicate field measurements, checking and calibration of equipment, participation in performanceevaluation programs, USGS project reviews, data verification, preparation and analysis of various quality-control samples (blanks and replicates), and laboratory quality-assurance programs. Water-level equipment, sampling equipment, and water-quality meters were checked regularly and calibrated in the field or office. All equipment used to measure field properties also was checked for accuracy through the USGS National Field Quality Assurance (NFQA) Program (Stanley and others, 1998) during 1996 through 1999.

All equipment used to collect study-area data was kept in locked USGS facilities. Sharing of this equipment with other sites was minimal to decrease the chance of crosscontamination from other sites. All 1993-1999 water-quality samples were kept in locked USGS facilities until the samples 
Table 4. Laboratory methods used by the U.S. Geological Survey National Water Quality Laboratory, 1994 through 1999.

[Method references provided in Stevens and others (2003); MRL, minimum reporting level, although dilutions for samples having high specific conductance resulted in higher MRL's for some samples; $\mu \mathrm{S} / \mathrm{cm}$, microsiemens per centimeter at 25 degrees Celsius; --, not applicable; $\mathrm{CaCO}_{3}$, calcium carbonate; mg/L, milligrams per liter; ${ }^{\circ} \mathrm{C}$, degrees Celsius; ASF, automated segmented-flow spectrophotometry; ICP, inductively coupled plasma; IC, ion chromatography; AA, atomic absorption spectrometry; $\mathrm{SiO}_{2}$, silicon dioxide; $\mathrm{N}$, nitrogen; $\mathrm{P}$, phosphorus; $\mu \mathrm{g} / \mathrm{L}$, micrograms per liter; $\mathrm{MS}$, mass spectroscopy; *, various]

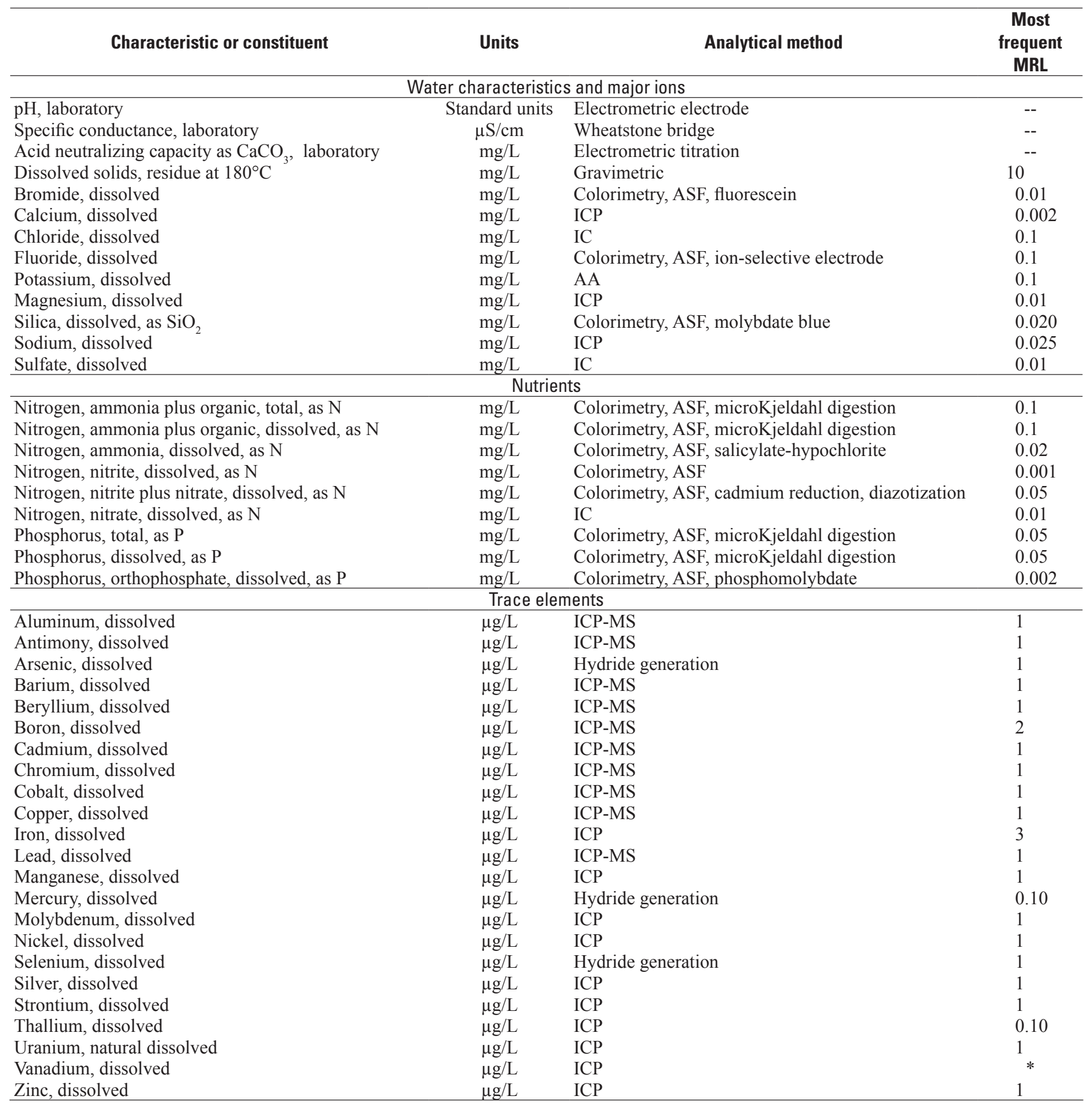


were delivered personally to the analyzing laboratory, except for DG-CFC samples collected in 1998, which were shipped to a USGS research laboratory in Virginia. Sample handling and custody (including sample delivery at the laboratory) was documented in project files.

All samples were submitted to the Metro District laboratory as double-blind samples. Sample bottles delivered to the laboratory were labeled with arbitrary sample numbers, not well numbers, so the laboratory staff did not know which sample had come from which well. In addition, the qualitycontrol samples (blanks and replicates, including splits) could not be distinguished visually from well samples. The actual sample identification (well number or quality-control sample type) was eventually provided to the Metro District, but only after all water-quality data for that sample were received by the USGS.

Replicate samples were collected in the field by the USGS to quantify variability in the groundwater data contributed by the groundwater, sampling and processing, field conditions, and laboratory conditions and analysis. The USGS usually collected two field replicates each sampling trip. Two types of replicate samples were collected: duplicates and splits. Duplicate samples indicate variability contributed by the environment (groundwater and site conditions), field processing, and laboratory processing and analysis; these samples were prepared by using all the same equipment to concurrently collect an extra sample during collection of the environmental sample. Bottles of a particular type were filled in sequence. For example, after the sample trace-elements bottle was filled, then the duplicate traceelements bottle was filled, then the sample nutrients bottle was filled, followed by the duplicate nutrients bottle, and so forth. Split samples indicate variability contributed by the laboratory only, and were prepared by compositing sample water for each bottle type, shaking the composite sample to mix, and then apportioning the composite between two bottle sets. The data from analysis of duplicate samples collected during 1993-1995 indicated that variability contributed by either the environment, sampling methods, field processing, or laboratory processing and analysis in the data was substantial, especially in the lowconcentration range.

When the groundwater-sampling method was changed in late 1995, the number and types of quality-control samples were expanded to provide more information about the bias and variability in the water-quality data that was contributed by the laboratory and to provide more quantitative information about low-concentration trace elements. From December 1995 through 1998, a special type of replicate sampling was done for at least one well each quarter to provide detailed information about natural groundwater variability (aquifer variation), and variability contributed by sampling, field processing, laboratory processing, and laboratory analysis. This sampling was called a "triplicate-split" sampling; four bottle sets were filled from the same well by making two replicate composites, each of which produced a pair of split samples. The first composite sample produced the environmental sample and a split of the environmental sample (sample sub-type $\mathrm{C} 1$ ), both of which were analyzed by the Metro District laboratory so the differences in reported values for the first split pair indicated variability contributed only by the processing and analysis at the Metro District laboratory. The sample pair from the second composite sampling (sample sub-type C2) was prepared immediately after the first composite sampling was bottled; the first of this split pair was analyzed by the Metro District laboratory, and the second sample of this split pair was analyzed by the NWQL. Thus, the differences in reported values for the second split pair represented only bias or variability between the two laboratories. Further performance-evaluation data are needed to determine which laboratory value was most accurate (least biased). The difference in reported values between composite 1 (the first split pair) and composite 2 (the second split pair) indicated variability contributed by the environment (groundwater and site conditions), field processing, and laboratory processing and analysis, just like other types of duplicate samples. This sampling was called a "triplicate-split" because three of the four bottle sets were sent to the Metro District laboratory for analysis, and a split of one of the bottle sets was sent to the NWQL for analysis. The data from the triplicate-split sampling indicated that variability in the groundwater data contributed by the laboratory processing and analysis at the Metro District generally was greater than the variability contributed by the environment or field processing (appendixes 3-5).

Blank samples were collected by the USGS and analyzed to quantify contamination, a type of high bias, contributed by specific field conditions, sampling equipment, and laboratory analysis. Note that not all the blank-sample data represent contamination that would affect the water-quality data; some data represent unusual field conditions or equipment combinations that affected few or none of the water-quality samples but provided helpful method information. The USGS usually collected one to three field-blank samples each quarter. Field blanks usually were prepared by passing deionized water (1993 through October 1995) or certified inorganic blank water (December 1995 through 1999) through all the sampling equipment, then processing and preserving like was done for a regular sample at a well site. In addition, equipment blanks occasionally were prepared at the USGS preparatory laboratory in Denver using selected equipment and analyzed as regular samples. Equipment blanks do not indicate bias in the sample data contributed by field conditions near Deer Trail because these samples were prepared at a USGS laboratory near Denver. Blank samples either were submitted to the Metro District laboratory or to the NWQL and usually were analyzed with the same analytical equipment as regular samples. However, a special low-level trace-element sample was analyzed by the NWQL on "blanks only" analytical equipment at least once a year from December 1995 through 1999 to quantify even very low levels of bias. The data from analysis of blank samples indicated that high bias in the low-concentration range generally was less than environmental concentrations (appendix 7).

The continuous-recorder sensors for specific conductance and groundwater temperature that were installed at well D6 during 1997 and 1998 were quality assured. The conductance sensor was calibrated annually and checked periodically for accuracy with standard solutions. The temperature sensor was 
checked at least annually for accuracy by comparing the output from the sensor with that from a certified thermometer in various water baths.

Little information was provided to the USGS about the analytical quality-assurance practices and procedures of the Metro District laboratory during 1993-1998. Ion balances were checked by the Metro District and used as rerun criteria before any data were released to the USGS (M.L. Castlebury, Metro District laboratory, oral commun., October 1995). The USGS was not provided the opportunity to request verification of analytical values or reanalysis of samples when data were anomalous, so the chemical data in this report may contain some laboratory errors, particularly for anomalously small or large concentration values. The Metro District laboratory participated in a laboratory review conducted by the USGS in late 1991; this laboratory was found to be "performing at an adequate level of operation" at that time (D.W. Erdmann, USGS, written commun., March 10, 1992).

The analytical quality-assurance practices and procedures for the NWQL during 1993-1999 are documented by various reports. The analytical quality-assurance practices and procedures of the NWQL are described by Friedman and Erdmann (1982). Analytical bias contributed by the NWQL also was evaluated for non-blank concentrations through USGS Blind Sample programs and performance-evaluation studies (Pirkey and Glodt, 1998). Variability in the water-quality data contributed by the NWQL also was evaluated through NWQL methodperformance programs (Pirkey and Glodt, 1998). Quality assurance of the NWQL was done at many levels. Field qualitycontrol samples (blanks and replicates analyzed by the NWQL) indicate bias and variability of the NWQL, as well as of field methods. The NWQL also had a three-tier quality-control process consisting of (1) method-performance evaluations (laboratory blanks, laboratory spikes, laboratory replicates, calibration standards, and calibration-check samples or standard reference materials), (2) data review and blind-sample programs, and (3) internal and external performance-evaluation studies (Pirkey and Glodt, 1998).

Both the Metro District laboratory and the NWQL participated in the USGS Standard Reference performance evaluation program during 1993-1999. This program was described by Farrar and Long (1996), and a similar report was published by the USGS for each set of performance-evaluation samples that were distributed. For this program, spiked natural-water solutions were distributed about twice each year to the participating laboratories for analysis, then the results were statistically analyzed and used to calculate the most probable value for the spiked solution. These performance-evaluation data (appendix 8) can indicate possible laboratory bias for constituents analyzed in the samples collected near Deer Trail during 1993-1999. However, laboratory methods and quality-assurance practices used for the performance-evaluation samples may have differed from those used for the samples collected near Deer Trail because these performance-evaluation samples were not submitted to the laboratories as Deer Trail project samples.

\section{Water Quality}

Water quality is determined by many processes. Chemical constituents can be added to the hydrologic system from transport in recharging water, dissolution of gases or rock minerals in the water, and chemical reactions that change one constituent into another. Chemical constituents can be subtracted from the hydrologic system by transport in discharging water, precipitation of constituents into solids, degassing of constituents into vapors, and chemical reactions. The occurrences and rates of these processes vary spatially and temporally. The result of all constituent additions and subtractions at a particular place for a particular time is described as the amount (mass) of that constituent in a given volume of water, or chemical concentration of the constituent. If more water is added to the hydrologic system but the mass of a constituent remains the same, the constituent is diluted and concentration decreases. Conversely, if water is removed from the hydrologic system (as in evaporation) but the mass of a constituent remains the same, the constituent is concentrated and concentration increases.

Because the mass of each constituent can vary in space, concentrations of a constituent can differ throughout a water body such as a pond or aquifer at any given time; constituents generally are not well mixed and uniformly distributed. Therefore, the selection of volume integrated to produce the discrete sample that was analyzed for chemistry affects the sample concentration. Well screens integrate groundwater over the length of the well screen, so samples from wells represent averaged concentrations; a specific geologic layer or aquifer subsample within the screened interval may actually have concentrations much higher or much lower than that of the sample. Some geologic layers (especially shale) within the screened area of a well may have high constituent concentrations but yield little water, whereas other geologic layers (especially sandstone or coarse alluvium) may have some zones of high concentrations and some zones of low concentrations but yield much water, which could result in a relatively dilute groundwater sample, depending on the contribution of each zone. Well D24 had a 30-ft screen because drilling of this borehole did not initially yield sufficient water volume for sampling, and no specific water-bearing zone could be isolated from this $30-\mathrm{ft}$ interval. Chemical-concentration data from this well may represent averaged concentrations from the entire $30-\mathrm{ft}$ screened interval, or may represent the chemistry of a few discrete water-yielding layers. In any case, the geologic (and therefore chemical and hydrologic) composition is not likely uniform over the entire $30 \mathrm{ft}$ screened for well D24 or even the entire $10 \mathrm{ft}$ screened for the other monitoring wells. Similar such considerations affect the representativeness of the water-quality samples collected from ponds, livestockwell troughs, and groundwater samples collected from well casings. Information for each sampled site is included in table 2 . 
Water quality can be characterized in terms of major ions, nutrients, trace elements, and bacteria. Major ions include calcium, chloride, magnesium, potassium, sodium, and sulfate and usually are contributed to water from dissolution of rock and mineral matter (Drever, 1988). Some major ions also can be contributed by leachate from sewage sludge (Gaggiani, 1991). Major ions can be removed from the water of the study area through precipitation of mineral phases or reaction. Nutrients include nitrogen and phosphorous constituents and can be contributed to the water of the study area from the atmosphere, decaying plant residue, rocks (such as shale), commercial fertilizers, animal feces, untreated human septage (perhaps associated with the homesteads), or biosolids (Yager and McMahon, 2012). Nutrients can be removed from the water of the study area through plant uptake, microbial reactions, and water transport. Trace elements are inorganic elements (including metals) that usually are present in natural waters at concentrations less than about $1 \mathrm{mg} / \mathrm{L}$. Many trace elements can be beneficial or detrimental to plant, animal, or human health depending on the form and concentration (Hem, 1992, p. 129). Trace elements dissolved in study-area water can be contributed from rocks, commercial fertilizers, and biosolids. Dissolved concentrations of trace elements can decrease from plant uptake, precipitation, water transport, or chemical reaction. Bacteria are microorganisms that are present in most waters and on many surfaces. Some bacteria are beneficial, some are harmful, and some are neither. Some bacteria are present only in specific environments. Fecal coliform and fecal streptococci bacteria in water originate from animal feces and human sewage (including biosolids) and indicate that harmful bacteria can be present (Hem, 1992).

The water-quality data for the study area that were collected during 1993-1999 are listed in tables 5-7. Data for inorganic water-quality samples collected during 1993-1998 and analyzed by the Metro District laboratory are listed in table 5. Note that the unusually small, censored (less than) values or unusually large values that were reported for the same site by the Metro District laboratory likely are laboratory errors. Data for bacteria samples (including associated quality-control data) are listed in table 6. The baseline data are included in tables 5 and 6 (all samples collected in 1993; see table 2 for sampling purpose). The reconnaissance data also are included in tables 5 and 6 (all groundwater and surface-water samples collected in 1993 and 1994; see table 2 for sampling purpose). The data resulting from routine sampling are included in tables 5 and 6 (all groundwater samples collected during 1995 through 1998). The data resulting from specialty sampling for pore water in the unsaturated zone during 1994 are included in table 5; no pore-water samples could be collected in 1995. The data resulting from specialty sampling for low-concentration trace elements in 1999 (including associated quality-control data) are listed in table 7; these data are for the groundwater samples that were collected during redox-indicator sampling. All inorganic water-quality data from this study were for dissolved constituents (from analysis of filtered samples) unless noted otherwise.
To determine if biosolids effects were present, an understanding of the uncertainty in the water-quality data is important. Water-quality data generally are presented in the form of tables of numbers, where a specific number in the table corresponds with the concentration value of a single constituent in a water sample collected from a specific site at a specific time. The numbers in these orderly tables give the impression that the concentration of the constituent is known precisely. However, the numbers presented as water-quality data are actually estimates of that constituent that would be more realistically represented as a range of numbers in a table, or a vertical bar instead of a point on a time-series graph. In fact, the actual or true concentration of any constituent is not known precisely because uncertainty is contributed to each number by laboratory methods, sampling methods, sampling equipment, field conditions, shipping or storage methods, and even by the variability in concentration of that constituent within the water being sampled. The amount of uncertainty contributed by each of these factors varies, which contributes to a unique total uncertainty (sometimes called "error") for each number presented in the water-quality data tables. Because the amount of uncertainty contributed by each factor varies, total uncertainty is difficult to quantify and even more difficult to present in a table or graph. Uncertainty can be described in terms of variability and bias in the data. Analyses of quality-control samples (such as replicates and blanks) provide information about the uncertainty (variability and bias) in the water-quality data. Differences between replicate samples indicate variability, and detections in blank samples plus participation in performance-evaluation programs indicate bias. Types of quality-control samples collected for the study area in 1993-1999 are discussed in the Approach section. The much stricter sampling protocols and the many more qualitycontrol samples implemented during 1995-1999 indicated that most of the variability and bias in the chemical data originated in the laboratory and was not from field processing or aquifer variation (inherently present in the aquifers). The qualitycontrol data indicated that variability in nitrate concentration, however, likely resulted from aquifer variation.

Nitrate can be analyzed directly in water samples, but for this study usually was analyzed as nitrite plus nitrate as nitrogen, where a separate analysis of nitrite indicated that most of the nitrogen was in the nitrate form. The 1993-1998 samples were analyzed for nitrite concentrations, as well as for nitrite plus nitrate concentrations. The laboratory data indicate that nitrite usually represented less than 10 percent of the nitrite plus nitrate concentration in groundwater samples from the study area, except where concentrations were very small (near the MRL where uncertainty is large) such as in samples from wells D15, D16, D21, D22, and D23. Even in samples from these groundwater sites, nitrite concentration usually was less than 50 percent of the nitrite-plus-nitrate concentration. Therefore, nitrite plus nitrate as nitrogen is referred to as nitrate in the remainder of this report. 
Table 5. Water-quality data for samples collected near Deer Trail, Colorado, 1993 through 1998.

[All data except field properties are for filtered samples analyzed at the Metro Wastewater Reclamation District laboratory; detailed well information is listed in appendix 1; associated quality-control data are included in appendixes 2-8; Temp., temperature; ${ }^{\circ} \mathrm{C}$, degrees Celsius; W.L. $\mathrm{ft}$ bmp, depth to groundwater in feet below measuring point; $\mathrm{mg} / \mathrm{L}$, milligrams per liter; $\mu \mathrm{S} / \mathrm{cm}$, microsiemens per centimeter at 25 degrees Celsius; $\mathrm{CaCO}_{3}$, calcium carbonate; $\mathrm{N}$, nitrogen; $\mathrm{P}$, phosphorus; $\mu \mathrm{g} / \mathrm{L}$, micrograms per liter; GW, groundwater; --, no data; <, less than; C1, first composite; c, calculated from calibration data; PW, pore water; ft, feet]

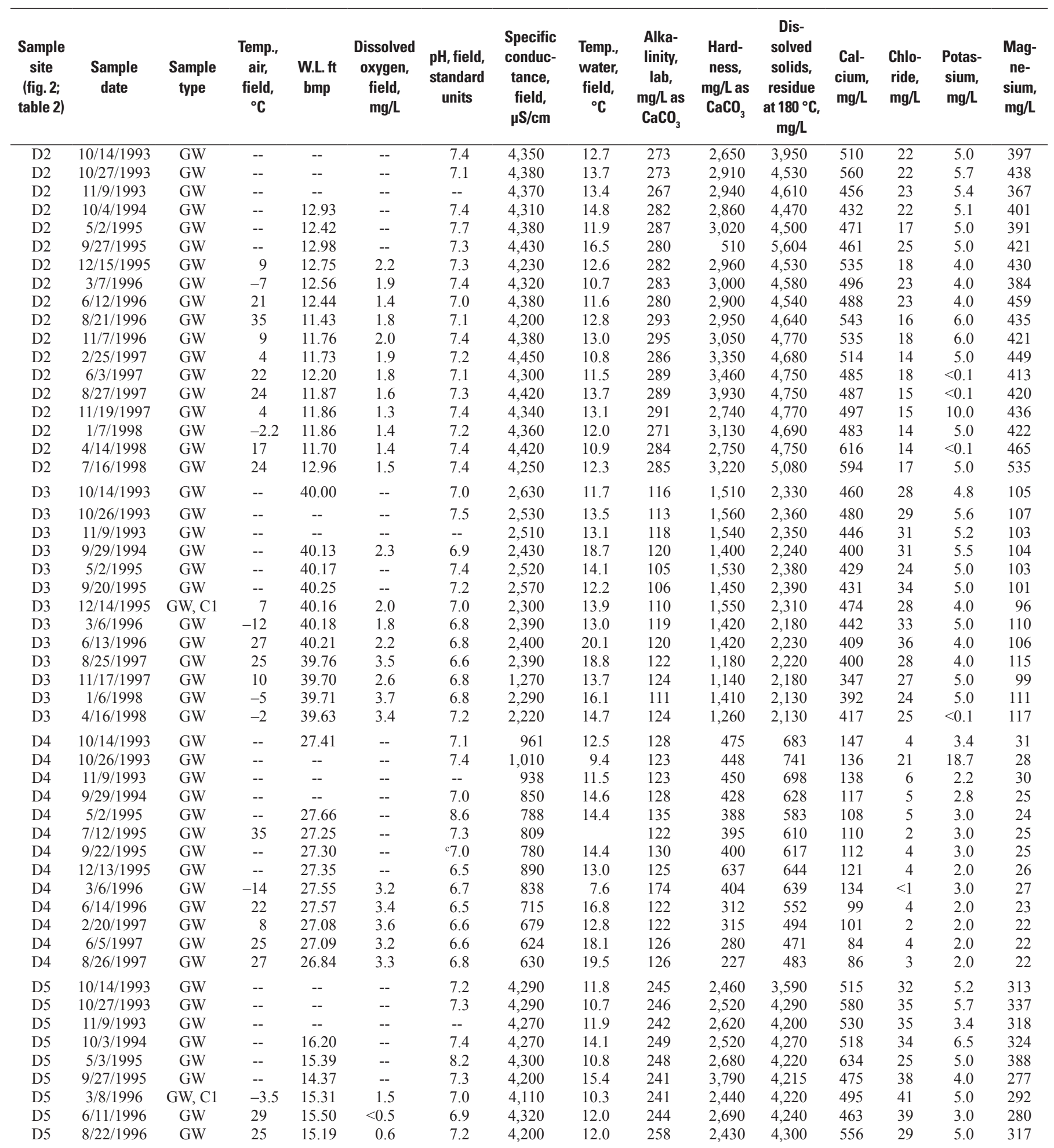


Table 5. Water-quality data for samples collected near Deer Trail, Colorado, 1993 through 1998.-Continued

[All data except field properties are for filtered samples analyzed at the Metro Wastewater Reclamation District laboratory; detailed well information is listed in appendix 1; associated quality-control data are included in appendixes 2-8; Temp., temperature; ${ }^{\circ} \mathrm{C}$, degrees Celsius; W.L. $\mathrm{ft}$ bmp, depth to groundwater in feet below measuring point; $\mathrm{mg} / \mathrm{L}$, milligrams per liter; $\mu \mathrm{S} / \mathrm{cm}$, microsiemens per centimeter at 25 degrees Celsius; $\mathrm{CaCO}_{3}$, calcium carbonate; $\mathrm{N}$, nitrogen; $\mathrm{P}$, phosphorus; $\mu \mathrm{g} / \mathrm{L}$, micrograms per liter; GW, groundwater; --, no data; <, less than; C1, first composite; c, calculated from calibration data; PW, pore water; ft, feet]

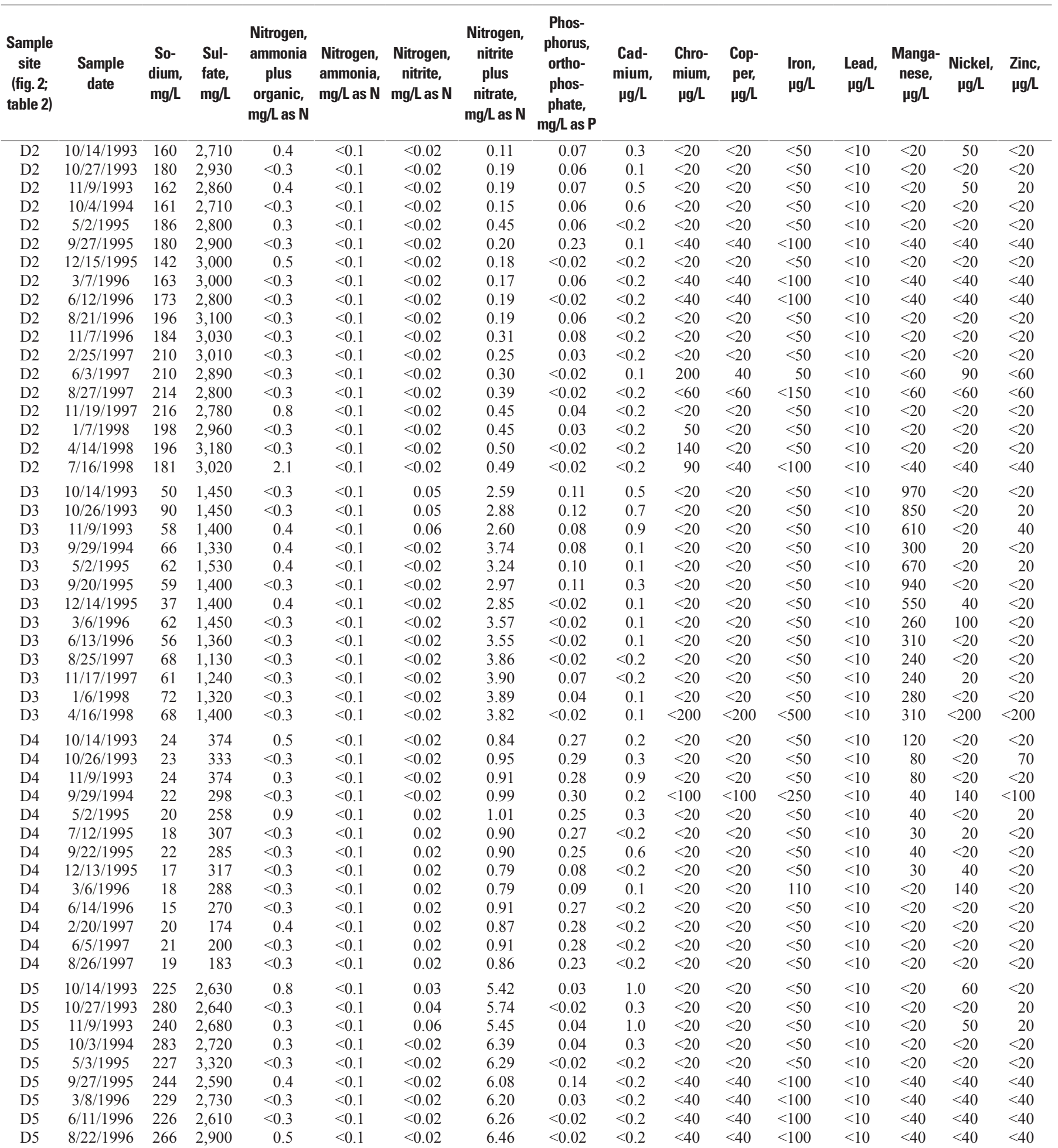


Table 5. Water-quality data for samples collected near Deer Trail, Colorado, 1993 through 1998.—Continued

[All data except field properties are for filtered samples analyzed at the Metro Wastewater Reclamation District laboratory; detailed well information is listed in appendix 1; associated quality-control data are included in appendixes 2-8; Temp., temperature; ${ }^{\circ} \mathrm{C}$, degrees Celsius; W.L. $\mathrm{ft}$ bmp, depth to groundwater in feet below measuring point; $\mathrm{mg} / \mathrm{L}$, milligrams per liter; $\mu \mathrm{S} / \mathrm{cm}$, microsiemens per centimeter at 25 degrees Celsius; $\mathrm{CaCO}_{3}$, calcium carbonate; $\mathrm{N}$, nitrogen; $\mathrm{P}$, phosphorus; $\mu \mathrm{g} / \mathrm{L}$, micrograms per liter; GW, groundwater; --, no data; <, less than; C1, first composite; c, calculated from calibration data; PW, pore water; ft, feet]

\begin{tabular}{|c|c|c|c|c|c|c|c|c|c|c|c|c|c|c|c|}
\hline $\begin{array}{c}\text { Sample } \\
\text { site } \\
\text { (fig. 2; } \\
\text { table 2) }\end{array}$ & $\begin{array}{l}\text { Sample } \\
\text { date }\end{array}$ & $\begin{array}{c}\text { Sample } \\
\text { type }\end{array}$ & $\begin{array}{l}\text { Temp., } \\
\text { air, } \\
\text { field, } \\
{ }^{\circ} \mathrm{C}\end{array}$ & $\begin{array}{l}\text { W.L. ft } \\
\text { bmp }\end{array}$ & $\begin{array}{c}\text { Dissolved } \\
\text { oxygen, } \\
\text { field, } \\
\text { mg/L }\end{array}$ & $\begin{array}{l}\text { pH, field, } \\
\text { standard } \\
\text { units }\end{array}$ & $\begin{array}{c}\text { Specific } \\
\text { conduc- } \\
\text { tance, } \\
\text { field, } \\
\mu \mathrm{S} / \mathrm{cm}\end{array}$ & $\begin{array}{l}\text { Temp., } \\
\text { water, } \\
\text { field, } \\
{ }^{\circ} \mathrm{C}\end{array}$ & $\begin{array}{c}\text { Alka- } \\
\text { linity, } \\
\text { lab, } \\
\mathrm{mg} / \mathrm{L} \text { as } \\
\mathrm{CaCO}_{3}\end{array}$ & $\begin{array}{c}\text { Hard- } \\
\text { ness, } \\
\mathrm{mg} / \mathrm{L} \text { as } \\
\mathrm{CaCO}_{3}\end{array}$ & $\begin{array}{c}\text { Dis- } \\
\text { solved } \\
\text { solids, } \\
\text { residue } \\
\text { at } 180^{\circ} \mathrm{C} \text {, } \\
\mathrm{mg} / \mathrm{L}\end{array}$ & $\begin{array}{l}\text { Cal- } \\
\text { cium, } \\
\text { mg/L }\end{array}$ & $\begin{array}{l}\text { Chlo- } \\
\text { ride, } \\
\mathrm{mg} / \mathrm{L}\end{array}$ & $\begin{array}{c}\text { Potas- } \\
\text { sium, } \\
\text { mg/L }\end{array}$ & $\begin{array}{c}\text { Mag- } \\
\text { ne- } \\
\text { sium, } \\
\text { mg/L }\end{array}$ \\
\hline D5 & $11 / 8 / 1996$ & GW & 10 & 15.02 & 1.1 & 7.2 & 4,100 & 12.3 & 249 & 2,960 & 4,290 & 576 & 30 & 5.0 & 336 \\
\hline D5 & $2 / 26 / 1997$ & GW & -2 & 15.36 & 0.7 & 7.0 & 4,220 & 10.9 & 242 & 2,630 & 4,300 & 550 & 31 & 5.0 & 253 \\
\hline D5 & $11 / 18 / 1997$ & GW & 7 & 15.35 & 0.6 & 7.3 & 4,230 & 12.4 & 244 & 2,200 & 4,290 & 504 & 32 & 6.0 & 325 \\
\hline D5 & $1 / 8 / 1998$ & GW & 9 & 15.42 & 1.0 & 7.4 & 4,150 & 11.6 & 236 & 2,630 & 4,330 & 518 & 29 & $<0.1$ & 349 \\
\hline D5 & $4 / 15 / 1998$ & GW & 7 & 15.72 & 1.1 & 7.3 & 4,290 & 10.2 & 236 & 2,650 & 4,310 & 482 & 29 & $<0.1$ & 269 \\
\hline D5 & $7 / 15 / 1998$ & GW & 34 & 15.99 & $<0.5$ & 7.1 & 4,280 & 13.3 & 241 & 2,480 & 4,400 & 600 & 31 & 4.0 & 297 \\
\hline D6 & $10 / 14 / 1993$ & GW & -- & -- & -- & 7.3 & 15,000 & 12.8 & 573 & 8,060 & 15,800 & 400 & 370 & 11.0 & 1,940 \\
\hline D6 & $10 / 26 / 1993$ & GW & -- & -- & -- & 7.4 & -- & 10.4 & 579 & 8,900 & 17,900 & 400 & 373 & 16.0 & 2,130 \\
\hline D6 & $3 / 7 / 1996$ & GW & -7 & 9.60 & 1.0 & 7.1 & 15,000 & 10.4 & 634 & 10,100 & 19,200 & 400 & 515 & 12.0 & 2,000 \\
\hline D6 & $6 / 11 / 1996$ & $\mathrm{GW}$ & 27 & 8.24 & $<0.5$ & 6.8 & 15,800 & 11.9 & 611 & 9,790 & 18,600 & 410 & 380 & 11.0 & 2,160 \\
\hline D6 & $8 / 22 / 1996$ & GW & 25 & 7.78 & 1.2 & 7.0 & -- & 11.5 & 614 & 9,680 & 19,100 & 418 & 187 & 12.0 & 2,150 \\
\hline D6 & $11 / 7 / 1996$ & GW & 10 & 7.90 & 0.5 & 7.2 & 15,200 & 11.4 & 624 & 10,000 & 19,400 & 447 & 210 & 12.0 & 2,290 \\
\hline D6 & 2/26/1997 & GW & 3 & 8.35 & 0.7 & 6.9 & 15,400 & 10.5 & 626 & 10,100 & 19,200 & 469 & 414 & 10.0 & 2,070 \\
\hline D6 & 6/4/1997 & GW & 24 & 8.68 & 0.5 & 7.0 & 15,600 & 11.3 & 632 & 13,100 & 19,300 & 413 & 402 & $<0.1$ & 1,990 \\
\hline D6 & 8/27/1997 & GW & 29 & 6.39 & 1.8 & 7.1 & 15,600 & 12.1 & 655 & 11,000 & 19,400 & 429 & 412 & $<0.1$ & 2,030 \\
\hline D6 & $11 / 19 / 1997$ & GW & -- & 6.77 & 0.8 & 7.3 & 15,300 & 11.8 & 634 & 10,200 & 19,700 & 417 & 443 & $<0.1$ & 1,980 \\
\hline D6 & $1 / 7 / 1998$ & GW & 3.3 & 6.84 & $<0.5$ & 7.0 & 15,300 & 11.5 & 593 & 10,200 & 19,500 & 383 & 410 & 9.0 & 2,160 \\
\hline D6 & $4 / 15 / 1998$ & GW & 7 & 5.86 & 0.7 & 7.2 & 15,300 & 10.2 & 606 & 10,400 & 19,800 & 538 & 370 & 10.0 & 2,210 \\
\hline D6 & $7 / 16 / 1998$ & GW & 26 & 8.04 & $<0.5$ & 7.2 & 15,100 & 12.3 & 649 & 10,286 & 21,000 & 491 & 497 & $<0.1$ & 2,250 \\
\hline D7 & $10 / 14 / 1993$ & GW & -- & -- & -- & 7.4 & 2,450 & 13.7 & 209 & 1,380 & 2,170 & 390 & 15 & 3.6 & 122 \\
\hline D7 & $10 / 27 / 1993$ & GW & -- & -- & -- & 7.3 & 2,410 & 13.2 & 202 & 1,415 & 2,220 & 400 & 16 & 11.2 & 123 \\
\hline D8 & $10 / 26 / 1993$ & GW & -- & -- & -- & 7.2 & 4,250 & 12.8 & 308 & 2,380 & 4,130 & 530 & 66 & 4.8 & 241 \\
\hline D8 & $11 / 9 / 1993$ & GW & -- & -- & -- & -- & 4,310 & 12.9 & 306 & 2,200 & 4,130 & 520 & 68 & 5.2 & 260 \\
\hline D8 & $9 / 29 / 1994$ & GW & -- & 10.96 & -- & 7.6 & 4,540 & 19.2 & 335 & 2,630 & 4,770 & 530 & 72 & 5.5 & 260 \\
\hline D8 & $5 / 2 / 1995$ & GW & -- & 10.66 & -- & 7.6 & 4,580 & 11.3 & 326 & 2,530 & 4,570 & 589 & 45 & 5.0 & 290 \\
\hline D8 & $7 / 12 / 1995$ & GW & 35 & 10.47 & -- & 7.2 & 4,530 & 13.3 & 326 & 2,490 & 4,490 & 538 & 68 & 5.0 & 264 \\
\hline D8 & $9 / 22 / 1995$ & GW & -- & 10.74 & -- & 7.1 & 4,320 & 10.8 & 325 & 2,520 & 4,489 & 555 & 66 & 5.0 & 265 \\
\hline D8 & $12 / 13 / 1995$ & GW & -- & 10.59 & -- & 7.0 & 4,280 & 12.0 & 331 & 2,450 & 4,490 & 596 & 61 & 5.0 & 264 \\
\hline D8 & $3 / 5 / 1996$ & GW & -- & 10.58 & 0.5 & 6.5 & 4,310 & 9.9 & 328 & 2,280 & 4,400 & 566 & 70 & 5.0 & 249 \\
\hline D8 & $6 / 11 / 1996$ & GW & 27 & 10.63 & $<0.5$ & 6.5 & 4,500 & 11.7 & 331 & 2,400 & 4,460 & 556 & 65 & 4.0 & 246 \\
\hline D8 & 8/27/1997 & GW & 38 & 9.31 & 0.5 & 7.1 & 4,040 & 13.0 & 319 & 2,410 & 4,210 & 527 & 47 & $<0.1$ & 255 \\
\hline D8 & $11 / 19 / 1997$ & GW & 4 & 8.66 & 0.9 & 6.9 & 4,100 & -- & 320 & 1,900 & 4,190 & 517 & 48 & 11.0 & 253 \\
\hline D8 & $1 / 7 / 1998$ & GW & 5 & 8.53 & $<0.5$ & 7.2 & 4,070 & 10.8 & 301 & 2,320 & 4,000 & 504 & 45 & 5.0 & 212 \\
\hline D8 & 4/14/1998 & $\mathrm{GW}$ & 17 & 8.34 & 0.6 & 7.1 & 4,040 & 9.3 & 303 & 2,300 & 3,970 & 566 & 42 & $<0.1$ & 218 \\
\hline D8 & $7 / 15 / 1998$ & GW & 35 & 9.91 & $<0.5$ & 6.9 & 3,960 & 12.6 & 300 & 2,320 & 3,980 & 584 & 42 & 4.0 & 214 \\
\hline
\end{tabular}


Table 5. Water-quality data for samples collected near Deer Trail, Colorado, 1993 through 1998.-Continued

[All data except field properties are for filtered samples analyzed at the Metro Wastewater Reclamation District laboratory; detailed well information is listed in appendix 1; associated quality-control data are included in appendixes 2-8; Temp., temperature; ${ }^{\circ} \mathrm{C}$, degrees Celsius; W.L. $\mathrm{ft}$ bmp, depth to groundwater in feet below measuring point; $\mathrm{mg} / \mathrm{L}$, milligrams per liter; $\mu \mathrm{S} / \mathrm{cm}$, microsiemens per centimeter at 25 degrees Celsius; $\mathrm{CaCO}_{3}$, calcium carbonate; $\mathrm{N}$, nitrogen; $\mathrm{P}$, phosphorus; $\mu \mathrm{g} / \mathrm{L}$, micrograms per liter; GW, groundwater; --, no data; <, less than; C1, first composite; c, calculated from calibration data; PW, pore water; ft, feet]

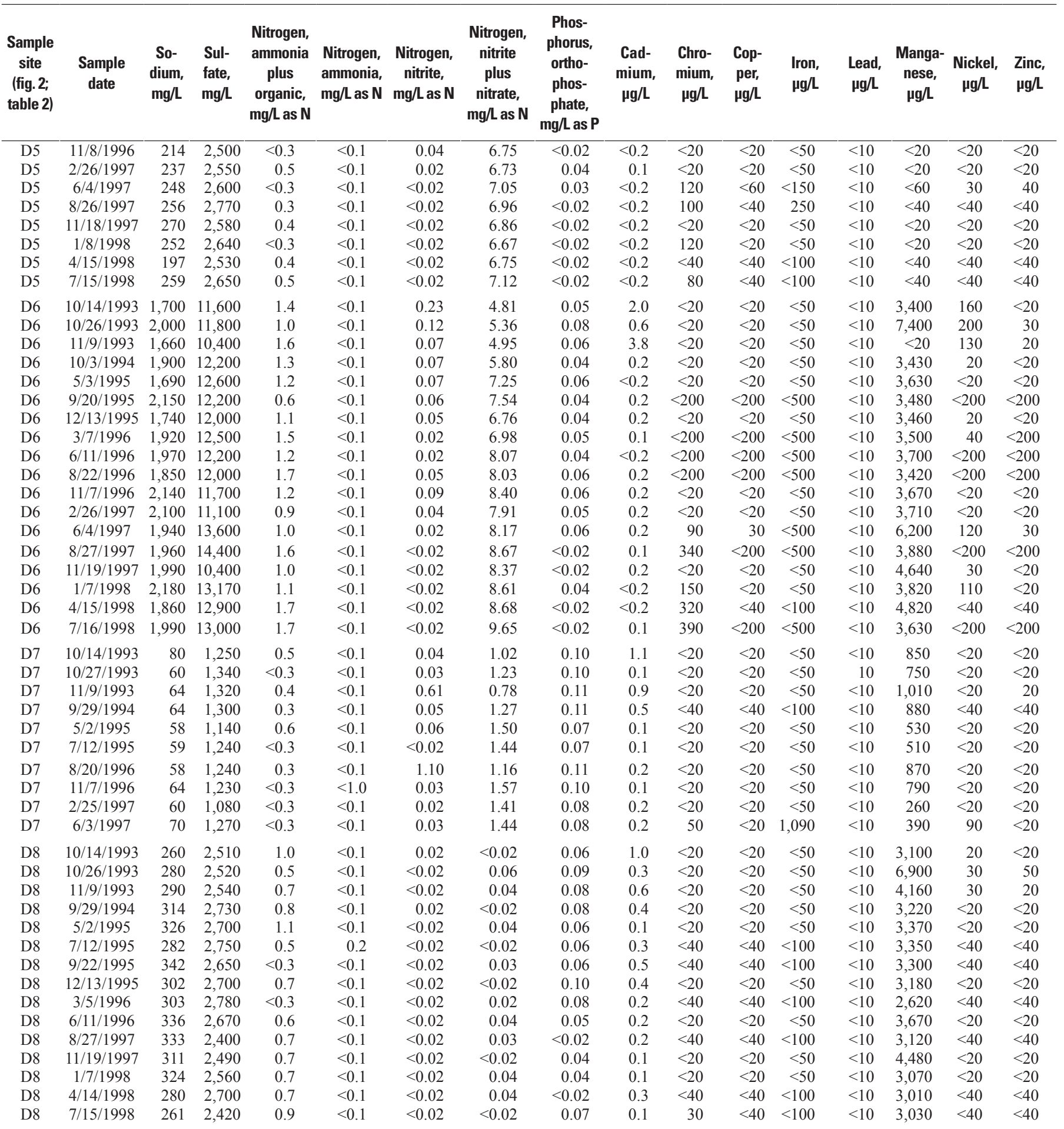


Table 5. Water-quality data for samples collected near Deer Trail, Colorado, 1993 through 1998.—Continued

[All data except field properties are for filtered samples analyzed at the Metro Wastewater Reclamation District laboratory; detailed well information is listed in appendix 1; associated quality-control data are included in appendixes 2-8; Temp., temperature; ${ }^{\circ} \mathrm{C}$, degrees Celsius; W.L. ft bmp, depth to groundwater in feet below measuring point; $\mathrm{mg} / \mathrm{L}$, milligrams per liter; $\mu \mathrm{S} / \mathrm{cm}$, microsiemens per centimeter at 25 degrees Celsius; $\mathrm{CaCO}_{3}$, calcium carbonate; $\mathrm{N}$, nitrogen; $\mathrm{P}$, phosphorus; $\mu \mathrm{g} / \mathrm{L}$, micrograms per liter; GW, groundwater; --, no data; <, less than; C1, first composite; c, calculated from calibration data; PW, pore water; ft, feet]

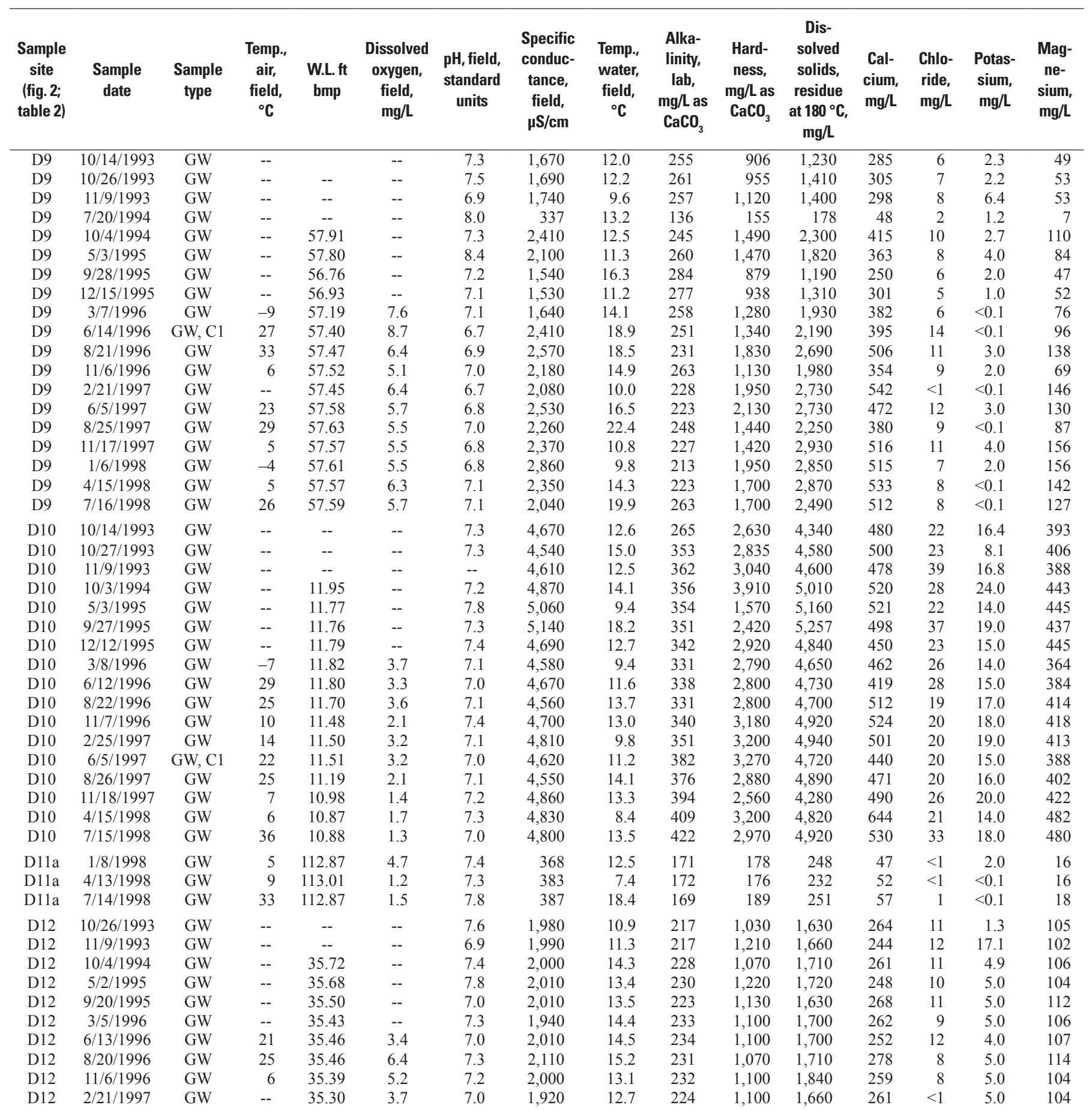


Table 5. Water-quality data for samples collected near Deer Trail, Colorado, 1993 through 1998.-Continued

[All data except field properties are for filtered samples analyzed at the Metro Wastewater Reclamation District laboratory; detailed well information is listed in appendix 1; associated quality-control data are included in appendixes 2-8; Temp., temperature; ${ }^{\circ} \mathrm{C}$, degrees Celsius; W.L. $\mathrm{ft}$ bmp, depth to groundwater in feet below measuring point; $\mathrm{mg} / \mathrm{L}$, milligrams per liter; $\mu \mathrm{S} / \mathrm{cm}$, microsiemens per centimeter at 25 degrees Celsius; $\mathrm{CaCO}_{3}$, calcium carbonate; $\mathrm{N}$, nitrogen; $\mathrm{P}$, phosphorus; $\mu \mathrm{g} / \mathrm{L}$, micrograms per liter; GW, groundwater; --, no data; <, less than; C1, first composite; c, calculated from calibration data; PW, pore water; ft, feet]

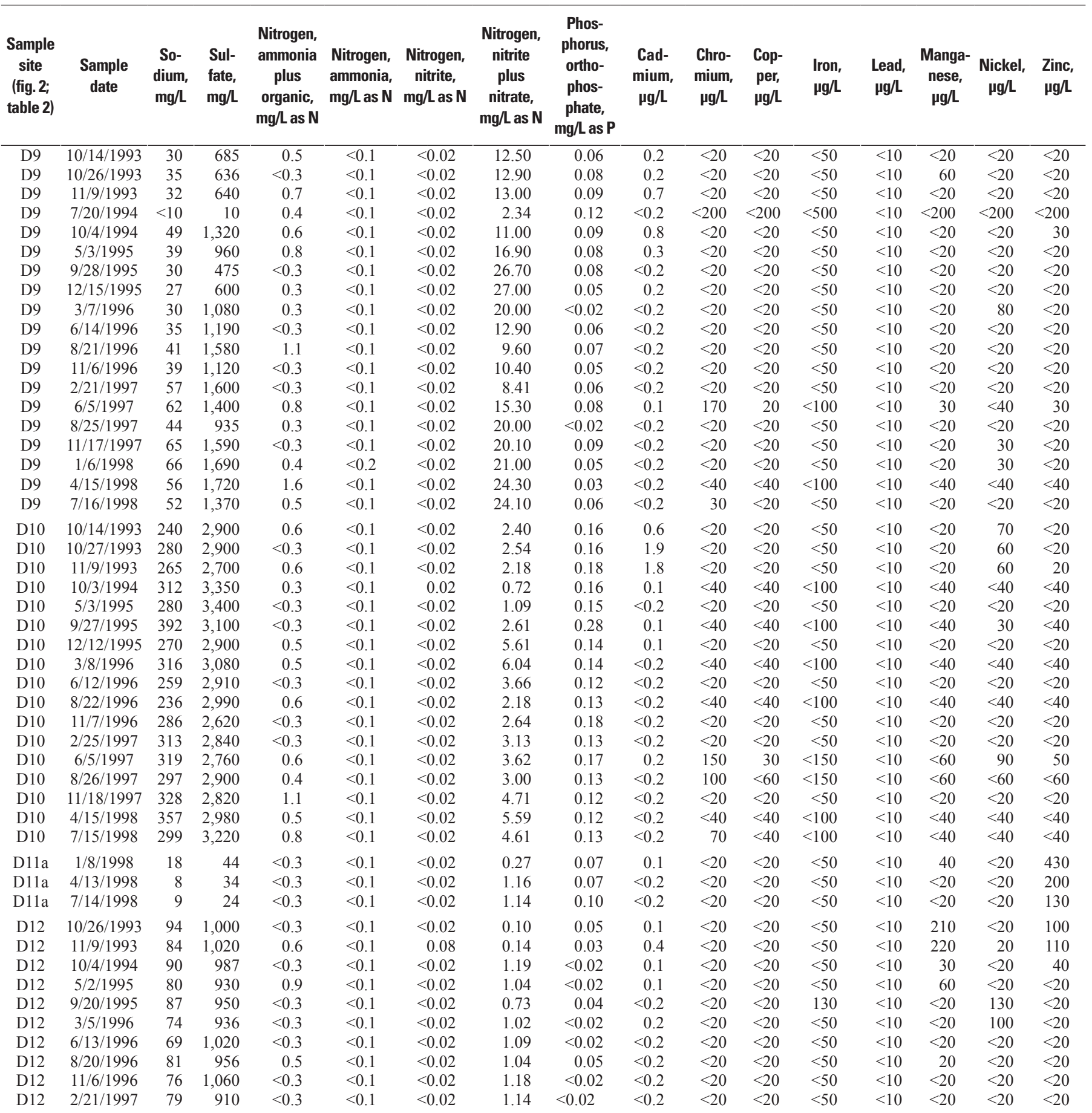


Table 5. Water-quality data for samples collected near Deer Trail, Colorado, 1993 through 1998.—Continued

[All data except field properties are for filtered samples analyzed at the Metro Wastewater Reclamation District laboratory; detailed well information is listed in appendix 1; associated quality-control data are included in appendixes 2-8; Temp., temperature; ${ }^{\circ} \mathrm{C}$, degrees Celsius; W.L. ft bmp, depth to groundwater in feet below measuring point; $\mathrm{mg} / \mathrm{L}$, milligrams per liter; $\mu \mathrm{S} / \mathrm{cm}$, microsiemens per centimeter at 25 degrees Celsius; $\mathrm{CaCO}_{3}$, calcium carbonate; $\mathrm{N}$, nitrogen; $\mathrm{P}$, phosphorus; $\mu \mathrm{g} / \mathrm{L}$, micrograms per liter; GW, groundwater; --, no data; <, less than; $\mathrm{Cl}$, first composite; c, calculated from calibration data; PW, pore water; ft, feet]

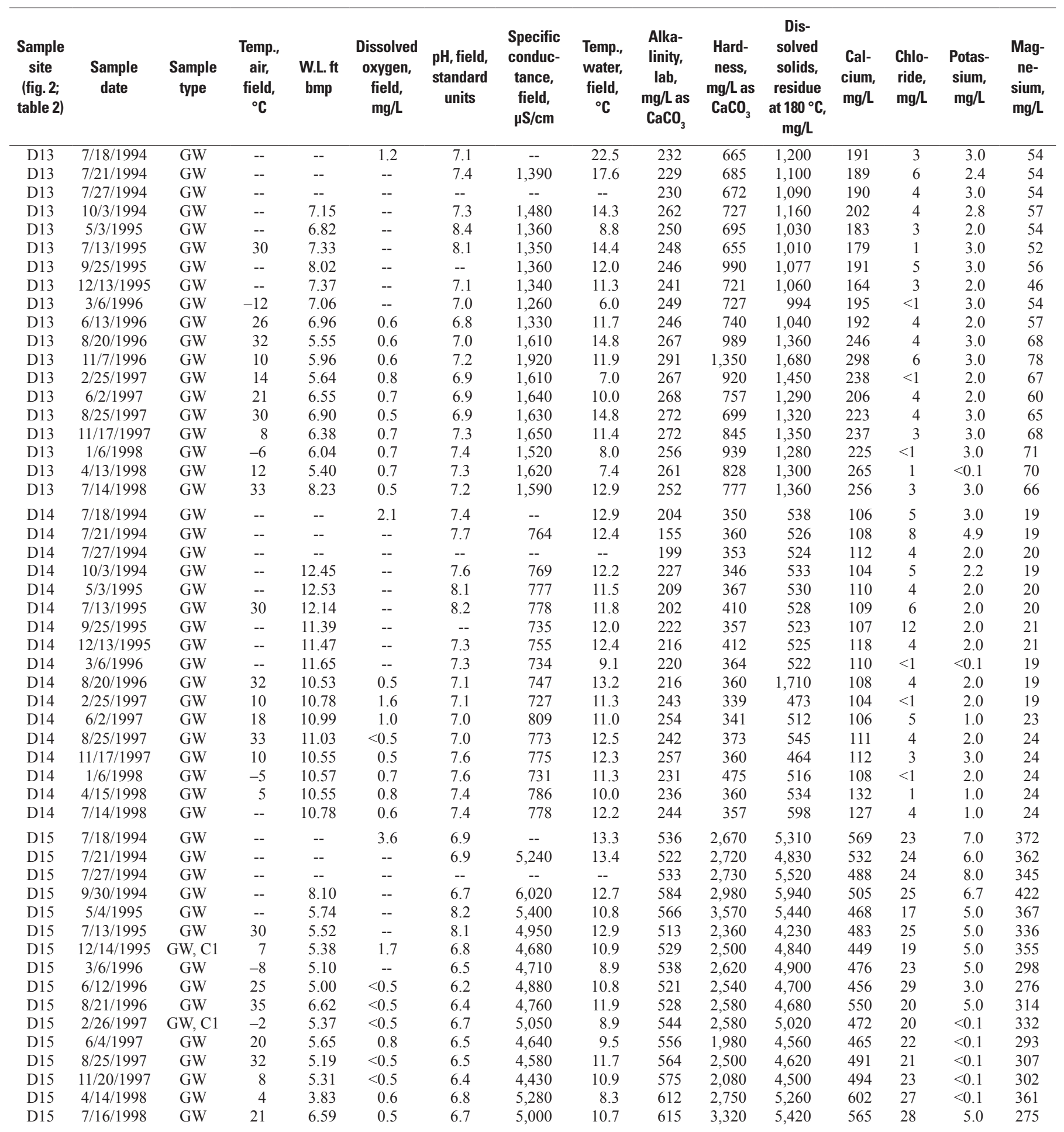


Table 5. Water-quality data for samples collected near Deer Trail, Colorado, 1993 through 1998.-Continued

[All data except field properties are for filtered samples analyzed at the Metro Wastewater Reclamation District laboratory; detailed well information is listed in appendix 1; associated quality-control data are included in appendixes 2-8; Temp., temperature; ${ }^{\circ} \mathrm{C}$, degrees Celsius; W.L. $\mathrm{ft}$ bmp, depth to groundwater in feet below measuring point; $\mathrm{mg} / \mathrm{L}$, milligrams per liter; $\mu \mathrm{S} / \mathrm{cm}$, microsiemens per centimeter at 25 degrees Celsius; $\mathrm{CaCO}_{3}$, calcium carbonate; $\mathrm{N}$, nitrogen; $\mathrm{P}$, phosphorus; $\mu \mathrm{g} / \mathrm{L}$, micrograms per liter; GW, groundwater; --, no data; <, less than; C1, first composite; c, calculated from calibration data; PW, pore water; ft, feet]

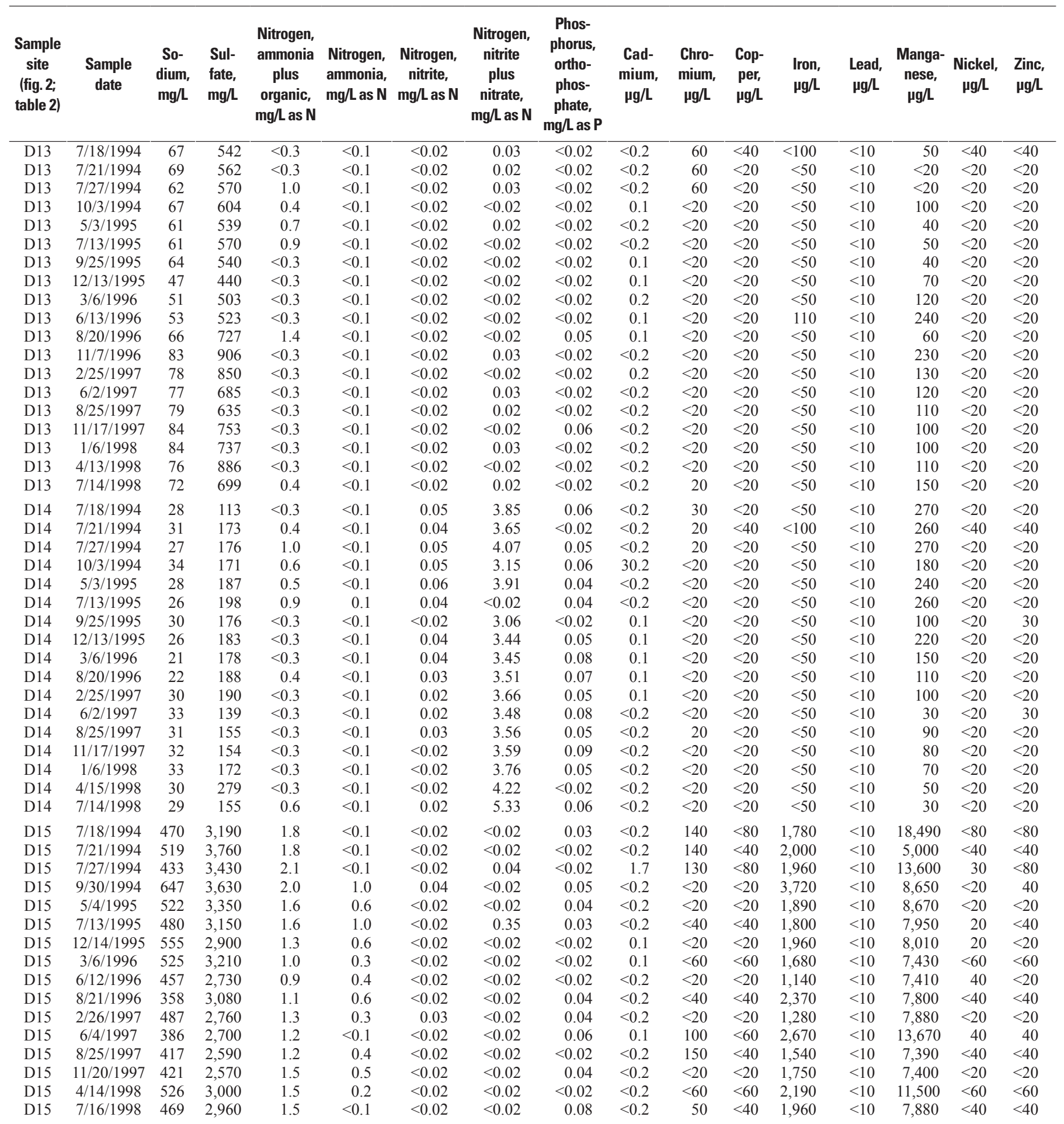


Table 5. Water-quality data for samples collected near Deer Trail, Colorado, 1993 through 1998.—Continued

[All data except field properties are for filtered samples analyzed at the Metro Wastewater Reclamation District laboratory; detailed well information is listed in appendix 1; associated quality-control data are included in appendixes 2-8; Temp., temperature; ${ }^{\circ} \mathrm{C}$, degrees Celsius; W.L. $\mathrm{ft}$ bmp, depth to groundwater in feet below measuring point; $\mathrm{mg} / \mathrm{L}$, milligrams per liter; $\mu \mathrm{S} / \mathrm{cm}$, microsiemens per centimeter at 25 degrees Celsius; $\mathrm{CaCO}_{3}$, calcium carbonate; $\mathrm{N}$, nitrogen; $\mathrm{P}$, phosphorus; $\mu \mathrm{g} / \mathrm{L}$, micrograms per liter; GW, groundwater; --, no data; <, less than; C1, first composite; c, calculated from calibration data; PW, pore water; ft, feet]

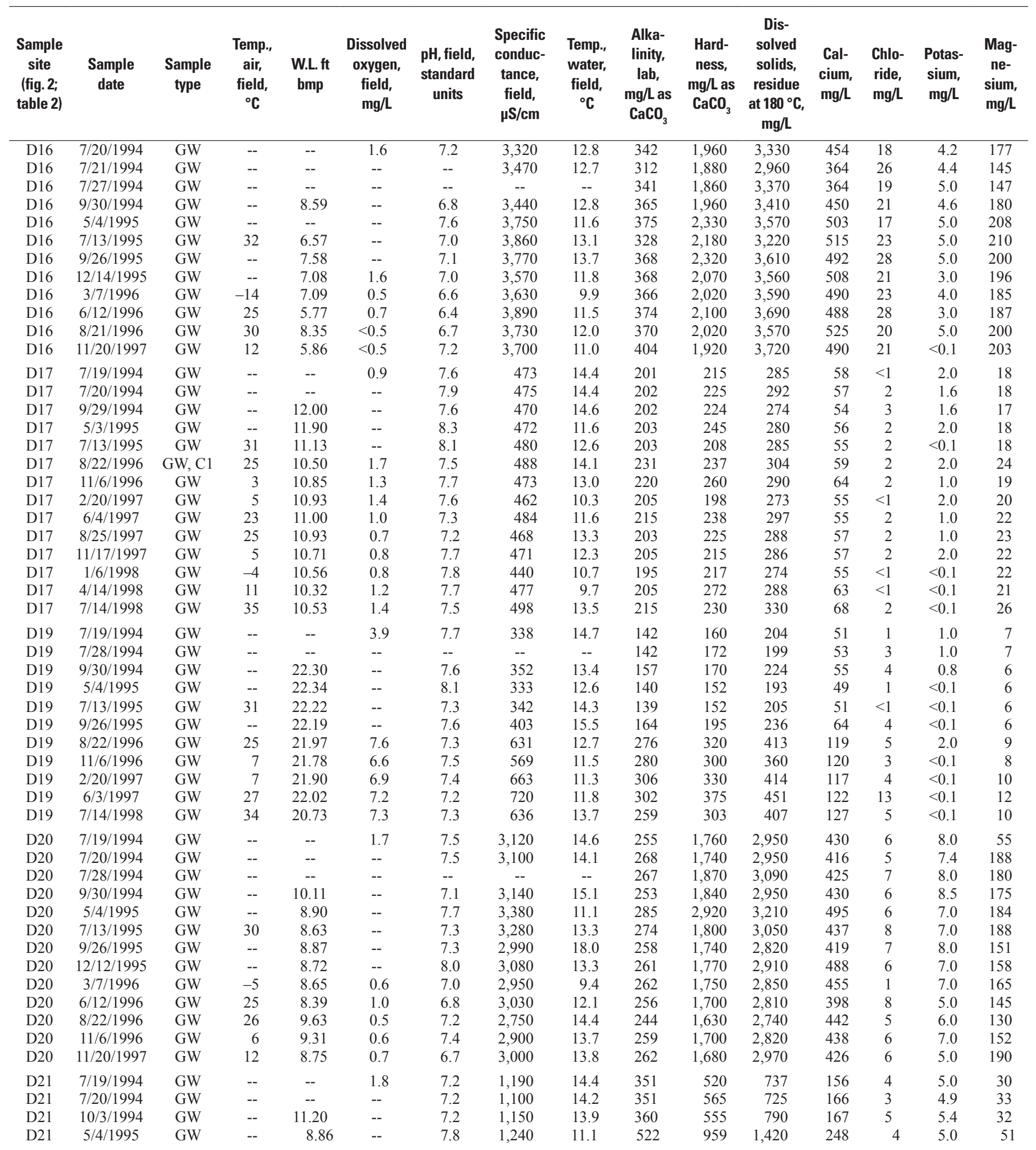


Table 5. Water-quality data for samples collected near Deer Trail, Colorado, 1993 through 1998._Continued

[All data except field properties are for filtered samples analyzed at the Metro Wastewater Reclamation District laboratory; detailed well information is listed in appendix 1; associated quality-control data are included in appendixes 2-8; Temp., temperature; ${ }^{\circ} \mathrm{C}$, degrees Celsius; W.L. $\mathrm{ft}$ bmp, depth to groundwater in feet below measuring point; $\mathrm{mg} / \mathrm{L}$, milligrams per liter; $\mu \mathrm{S} / \mathrm{cm}$, microsiemens per centimeter at 25 degrees Celsius; $\mathrm{CaCO}_{3}$, calcium carbonate; $\mathrm{N}$, nitrogen; $\mathrm{P}$, phosphorus; $\mu \mathrm{g} / \mathrm{L}$, micrograms per liter; GW, groundwater; --, no data; <, less than; C1, first composite; c, calculated from calibration data; PW, pore water; ft, feet]

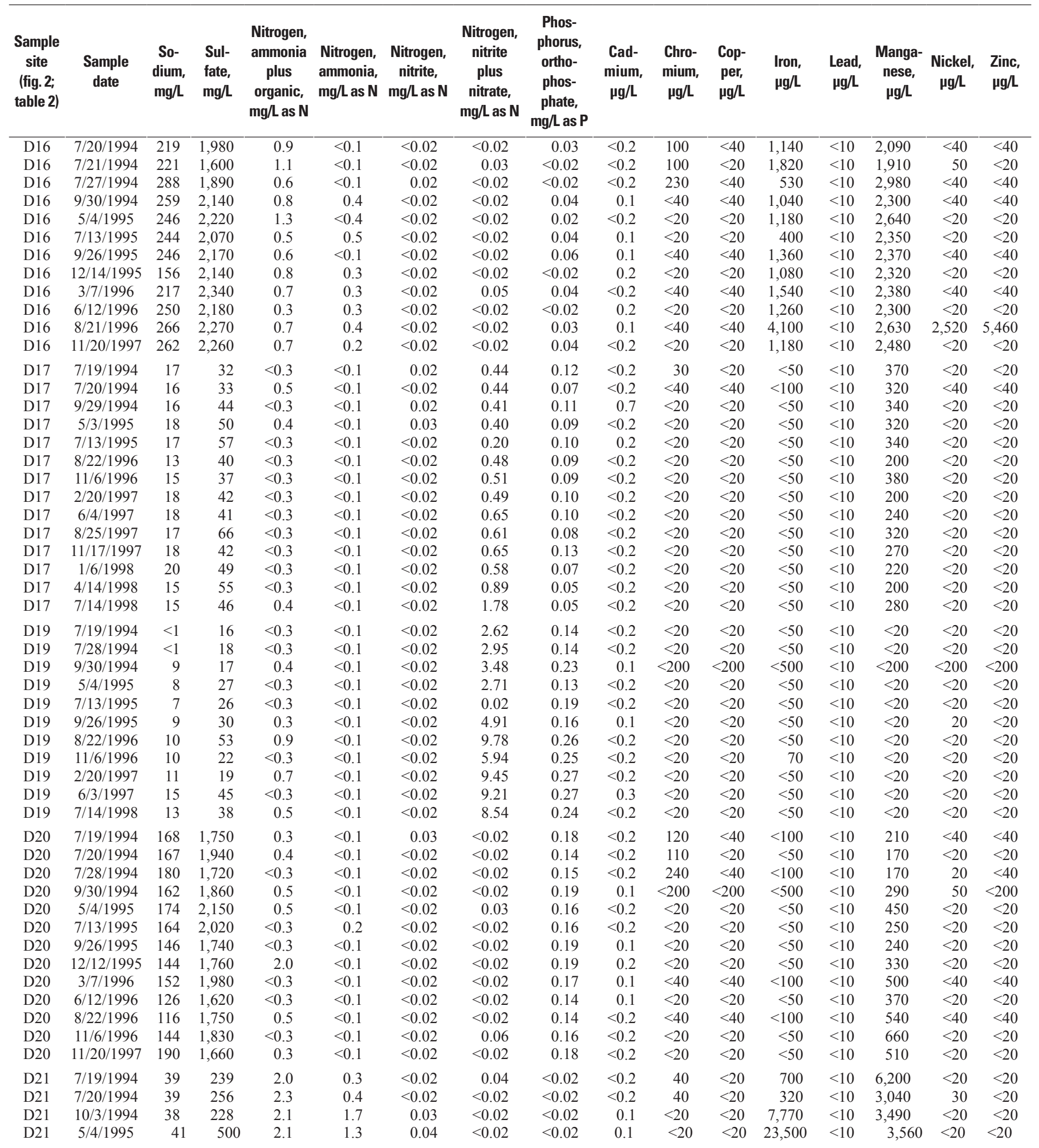


Table 5. Water-quality data for samples collected near Deer Trail, Colorado, 1993 through 1998.—Continued

[All data except field properties are for filtered samples analyzed at the Metro Wastewater Reclamation District laboratory; detailed well information is listed in appendix 1; associated quality-control data are included in appendixes 2-8; Temp., temperature; ${ }^{\circ} \mathrm{C}$, degrees Celsius; W.L. $\mathrm{ft}$ bmp, depth to groundwater in feet below measuring point; $\mathrm{mg} / \mathrm{L}$, milligrams per liter; $\mu \mathrm{S} / \mathrm{cm}$, microsiemens per centimeter at 25 degrees Celsius; $\mathrm{CaCO}_{3}$, calcium carbonate; $\mathrm{N}$, nitrogen; $\mathrm{P}$, phosphorus; $\mu \mathrm{g} / \mathrm{L}$, micrograms per liter; GW, groundwater; --, no data; <, less than; C1, first composite; c, calculated from calibration data; PW, pore water; ft, feet]

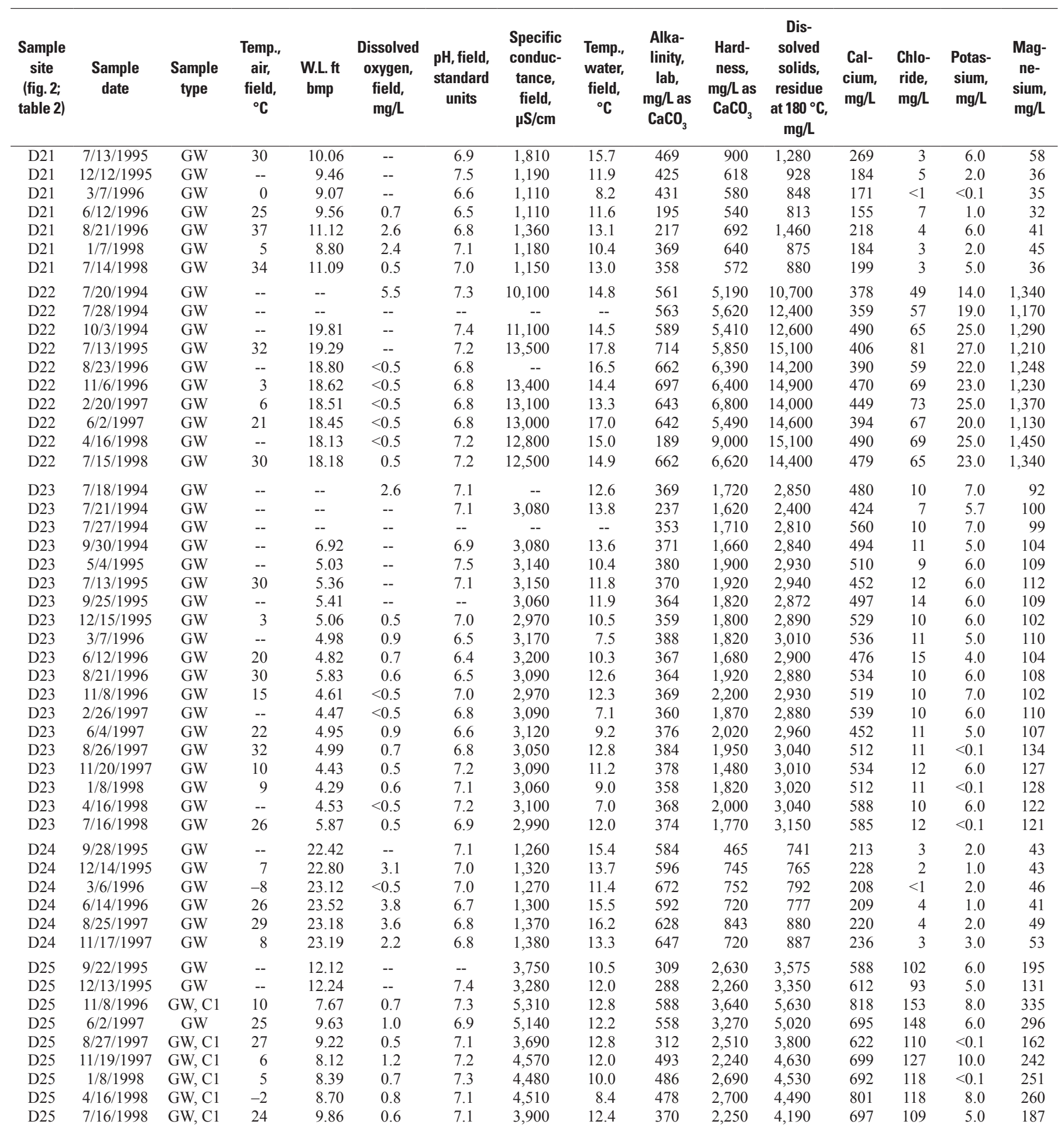


Table 5. Water-quality data for samples collected near Deer Trail, Colorado, 1993 through 1998.-Continued

[All data except field properties are for filtered samples analyzed at the Metro Wastewater Reclamation District laboratory; detailed well information is listed in appendix 1; associated quality-control data are included in appendixes 2-8; Temp., temperature; ${ }^{\circ} \mathrm{C}$, degrees Celsius; W.L. $\mathrm{ft}$ bmp, depth to groundwater in feet below measuring point; $\mathrm{mg} / \mathrm{L}$, milligrams per liter; $\mu \mathrm{S} / \mathrm{cm}$, microsiemens per centimeter at 25 degrees Celsius; $\mathrm{CaCO}_{3}$, calcium carbonate; $\mathrm{N}$, nitrogen; $\mathrm{P}$, phosphorus; $\mu \mathrm{g} / \mathrm{L}$, micrograms per liter; GW, groundwater; --, no data; <, less than; C1, first composite; c, calculated from calibration data; PW, pore water; ft, feet]

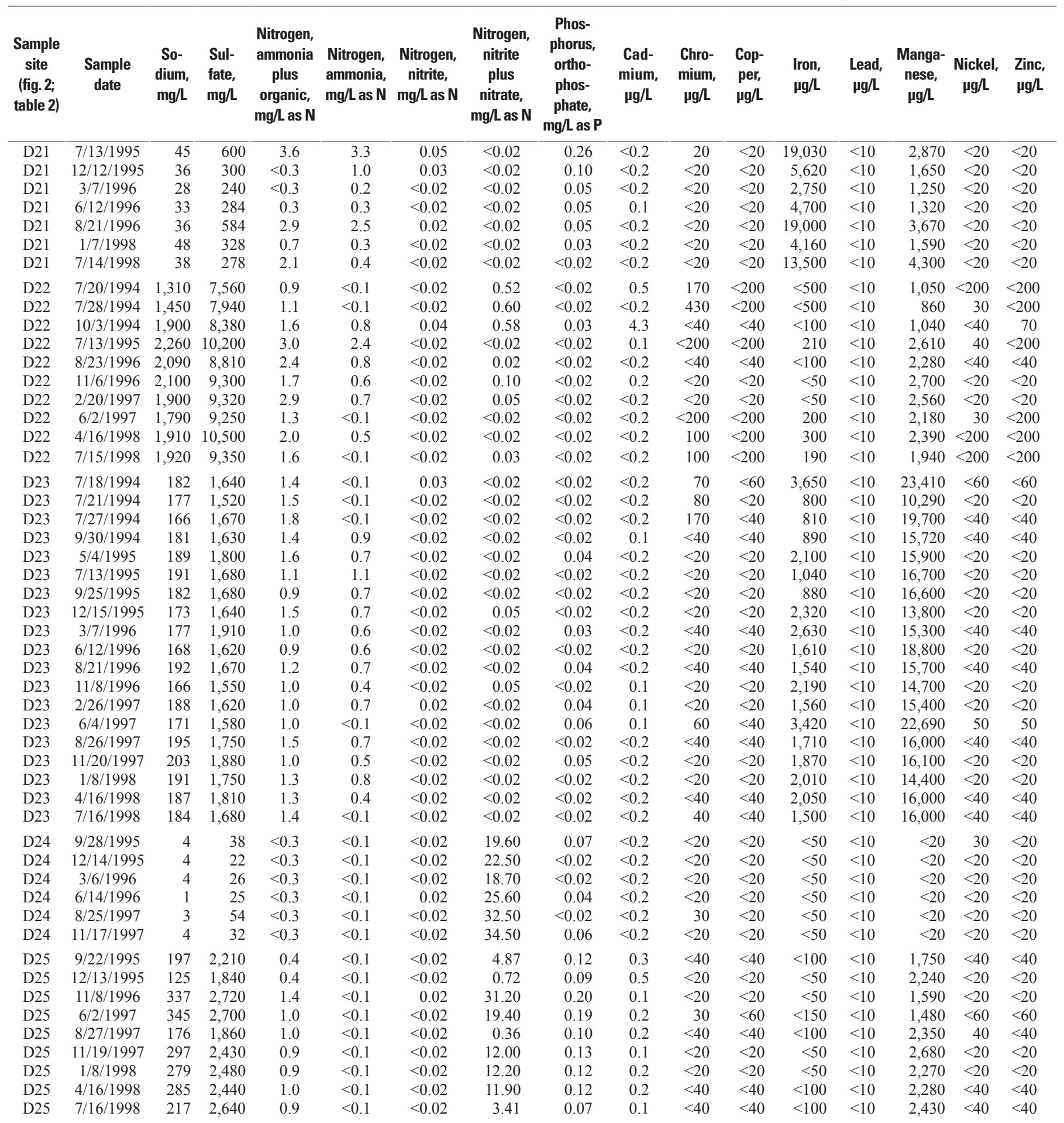


Table 5. Water-quality data for samples collected near Deer Trail, Colorado, 1993 through 1998.—Continued

[All data except field properties are for filtered samples analyzed at the Metro Wastewater Reclamation District laboratory; detailed well information is listed in appendix 1; associated quality-control data are included in appendixes 2-8; Temp., temperature; ${ }^{\circ} \mathrm{C}$, degrees Celsius; W.L. $\mathrm{ft}$ bmp, depth to groundwater in feet below measuring point; $\mathrm{mg} / \mathrm{L}$, milligrams per liter; $\mu \mathrm{S} / \mathrm{cm}$, microsiemens per centimeter at 25 degrees Celsius; $\mathrm{CaCO}_{3}$, calcium carbonate; $\mathrm{N}$, nitrogen; $\mathrm{P}$, phosphorus; $\mu \mathrm{g} / \mathrm{L}$, micrograms per liter; GW, groundwater; --, no data; <, less than; C1, first composite; c, calculated from calibration data; PW, pore water; ft, feet]

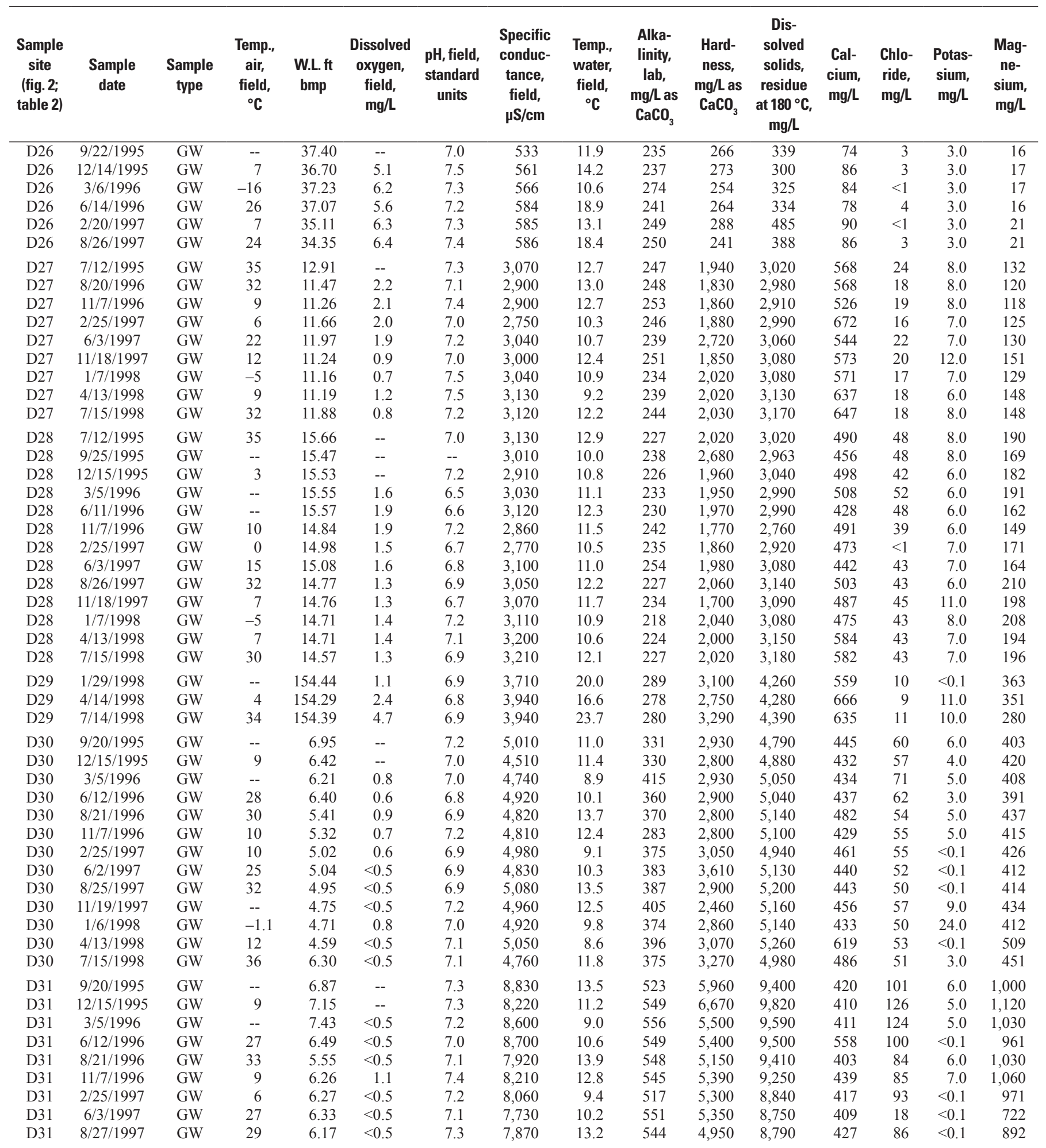


Table 5. Water-quality data for samples collected near Deer Trail, Colorado, 1993 through 1998.-Continued

[All data except field properties are for filtered samples analyzed at the Metro Wastewater Reclamation District laboratory; detailed well information is listed in appendix 1; associated quality-control data are included in appendixes 2-8; Temp., temperature; ${ }^{\circ} \mathrm{C}$, degrees Celsius; W.L. $\mathrm{ft}$ bmp, depth to groundwater in feet below measuring point; $\mathrm{mg} / \mathrm{L}$, milligrams per liter; $\mu \mathrm{S} / \mathrm{cm}$, microsiemens per centimeter at 25 degrees Celsius; $\mathrm{CaCO}_{3}$, calcium carbonate; $\mathrm{N}$, nitrogen; $\mathrm{P}$, phosphorus; $\mu \mathrm{g} / \mathrm{L}$, micrograms per liter; GW, groundwater; --, no data; <, less than; C1, first composite; c, calculated from calibration data; PW, pore water; ft, feet]

\begin{tabular}{|c|c|c|c|c|c|c|c|c|c|c|c|c|c|c|c|c|}
\hline $\begin{array}{l}\text { Sample } \\
\text { site } \\
\text { (fig. 2; } \\
\text { table 2) }\end{array}$ & $\begin{array}{c}\text { Sample } \\
\text { date }\end{array}$ & $\begin{array}{l}\text { So- } \\
\text { dium, } \\
\text { mg/L }\end{array}$ & $\begin{array}{l}\text { Sul- } \\
\text { fate, } \\
\mathrm{mg} / \mathrm{L}\end{array}$ & $\begin{array}{c}\text { Nitrogen, } \\
\text { ammonia } \\
\text { plus } \\
\text { organic, } \\
\mathrm{mg} / \mathrm{L} \text { as } \mathrm{N}\end{array}$ & $\begin{array}{l}\text { Nitrogen, } \\
\text { ammonia, } \\
\text { mg/L as N }\end{array}$ & $\begin{array}{c}\text { Nitrogen, } \\
\text { nitrite, } \\
\text { mg/L as } N\end{array}$ & $\begin{array}{c}\text { Nitrogen, } \\
\text { nitrite } \\
\text { plus } \\
\text { nitrate, } \\
\text { mg/L as N }\end{array}$ & $\begin{array}{l}\text { Phos- } \\
\text { phorus, } \\
\text { ortho- } \\
\text { phos- } \\
\text { phate, } \\
\text { mg/L as P }\end{array}$ & $\begin{array}{c}\text { Cad- } \\
\text { mium, } \\
\mu \mathrm{g} / \mathrm{L}\end{array}$ & $\begin{array}{c}\text { Chro- } \\
\text { mium, } \\
\mu \mathrm{g} / \mathrm{L}\end{array}$ & $\begin{array}{l}\text { Cop- } \\
\text { per, } \\
\mu g / L\end{array}$ & $\begin{array}{l}\text { Iron, } \\
\mu \mathrm{g} / \mathrm{L}\end{array}$ & $\begin{array}{l}\text { Lead, } \\
\mu \mathrm{g} / \mathrm{L}\end{array}$ & $\begin{array}{c}\text { Manga- } \\
\text { nese, } \\
\mu \mathrm{g} / \mathrm{L}\end{array}$ & $\begin{array}{c}\text { Nickel, } \\
\mu \mathrm{g} / \mathrm{L}\end{array}$ & $\begin{array}{l}\text { Zinc, } \\
\mu \mathrm{g} / \mathrm{L}\end{array}$ \\
\hline D26 & $9 / 22 / 1995$ & 13 & 37 & $<0.3$ & $<0.1$ & $<0.02$ & 4.98 & 0.12 & 0.1 & $<20$ & $<20$ & $<50$ & $<10$ & $<20$ & $<20$ & $<20$ \\
\hline D26 & $12 / 14 / 1995$ & 10 & 38 & $<0.3$ & $<0.1$ & $<0.02$ & 4.95 & 0.07 & $<0.2$ & $<20$ & $<20$ & $<50$ & $<10$ & $<20$ & 50 & $<20$ \\
\hline D26 & $2 / 20 / 1997$ & 14 & 47 & $<0.3$ & $<0.1$ & $<0.02$ & 4.57 & 0.09 & $<0.2$ & $<20$ & $<20$ & $<50$ & $<10$ & $<20$ & $<20$ & $<20$ \\
\hline D26 & $8 / 26 / 1997$ & 13 & 57 & 0.5 & $<0.1$ & $<0.02$ & 5.39 & 0.06 & $<0.2$ & $<20$ & $<20$ & $<50$ & $<10$ & $<20$ & $<20$ & $<20$ \\
\hline D27 & $7 / 12 / 1995$ & 77 & 1,790 & 0.8 & 0.1 & $<0.02$ & 0.35 & 0.57 & $<0.2$ & $<20$ & $<20$ & $<50$ & $<10$ & 310 & $<20$ & $<20$ \\
\hline $\mathrm{D} 27$ & $8 / 20 / 1996$ & 71 & 1,740 & 1.3 & $<0.1$ & $<0.02$ & 0.31 & 0.38 & 0.1 & $<20$ & $<20$ & $<50$ & $<10$ & $<20$ & $<20$ & $<20$ \\
\hline D27 & $11 / 7 / 1996$ & 73 & 1,610 & $<0.3$ & $<0.1$ & $<0.02$ & 0.55 & 0.38 & 0.3 & $<20$ & $<20$ & $<50$ & $<10$ & $<20$ & $<20$ & $<20$ \\
\hline D27 & $2 / 25 / 1997$ & 81 & 2,120 & $<0.3$ & $<0.1$ & $<0.02$ & 0.52 & 0.29 & 0.2 & $<20$ & $<20$ & $<50$ & $<10$ & $<20$ & $<20$ & $<20$ \\
\hline D27 & 6/3/1997 & 86 & 1,670 & $<0.3$ & $<0.1$ & $<0.02$ & 0.66 & 0.28 & $<0.2$ & 40 & 40 & 1,010 & $<10$ & 30 & 30 & $<40$ \\
\hline D28 & $7 / 12 / 1995$ & 86 & 1,810 & $<0.3$ & 0.2 & $<0.02$ & 4.05 & 0.04 & $<0.2$ & $<20$ & $<20$ & $<50$ & $<10$ & 220 & $<20$ & $<20$ \\
\hline D28 & 9/25/1995 & 78 & 1,780 & $<0.3$ & $<0.1$ & $<0.02$ & 5.66 & $<0.02$ & 0.1 & $<20$ & $<20$ & $<50$ & $<10$ & 70 & $<20$ & $<20$ \\
\hline $\mathrm{D} 28$ & $12 / 15 / 1995$ & 72 & 1,780 & $<0.3$ & $<0.1$ & $<0.02$ & 4.87 & $<0.02$ & 0.1 & $<20$ & $<20$ & $<50$ & $<10$ & 30 & $<20$ & $<20$ \\
\hline D28 & $3 / 5 / 1996$ & 90 & 1,890 & $<0.3$ & $<0.1$ & $<0.02$ & 5.25 & 0.04 & 0.1 & $<40$ & $<40$ & $<100$ & $<10$ & $<40$ & $<40$ & $<40$ \\
\hline D28 & $6 / 11 / 1996$ & 74 & 1,580 & $<0.3$ & $<0.1$ & $<0.02$ & 5.81 & $<0.02$ & $<0.2$ & $<20$ & $<20$ & $<50$ & $<10$ & $<20$ & $<20$ & $<20$ \\
\hline D28 & $11 / 7 / 1996$ & 81 & 1,700 & $<0.3$ & $<0.1$ & $<0.02$ & 7.55 & 0.08 & $<0.2$ & $<20$ & $<20$ & $<50$ & $<10$ & 20 & $<20$ & $<20$ \\
\hline D28 & $2 / 25 / 1997$ & 89 & 1,670 & $<0.3$ & $<0.1$ & $<0.02$ & 9.53 & 0.03 & 0.1 & $<20$ & $<20$ & $<50$ & $<10$ & $<20$ & $<20$ & $<20$ \\
\hline D28 & 6/3/1997 & 94 & 1,760 & $<0.3$ & $<0.1$ & $<0.02$ & 6.13 & 0.04 & 0.1 & 120 & $<40$ & 1,290 & $<10$ & 50 & 40 & 20 \\
\hline D28 & $8 / 26 / 1997$ & 101 & 1,800 & 0.2 & $<0.1$ & $<0.02$ & 6.06 & $<0.02$ & $<0.2$ & $<40$ & $<40$ & $<100$ & $<10$ & $<40$ & $<40$ & $<40$ \\
\hline D28 & $11 / 18 / 1997$ & 103 & 1,800 & 1.8 & $<0.1$ & $<0.02$ & 5.81 & $<0.02$ & $<0.2$ & $<20$ & $<20$ & $<50$ & $<10$ & $<20$ & $<20$ & $<20$ \\
\hline D28 & $1 / 7 / 1998$ & 88 & 1,890 & 0.3 & $<0.1$ & $<0.02$ & 5.14 & $<0.02$ & $<0.2$ & $<20$ & $<20$ & $<50$ & $<10$ & 60 & 70 & 60 \\
\hline $\mathrm{D} 28$ & $4 / 13 / 1998$ & 90 & 1,990 & $<0.3$ & $<0.1$ & $<0.02$ & 4.99 & $<0.02$ & $<0.2$ & $<40$ & $<40$ & $<100$ & $<10$ & $<40$ & $<40$ & $<40$ \\
\hline D28 & $7 / 15 / 1998$ & 97 & 1,880 & 0.5 & $<0.1$ & $<0.02$ & 6.74 & $<0.02$ & $<0.2$ & 40 & $<40$ & $<100$ & $<10$ & $<40$ & $<40$ & $<40$ \\
\hline D30 & 2/25/1997 & 383 & 3,060 & $<0.3$ & $<0.1$ & $<0.02$ & $<0.02$ & 0.10 & 0.1 & $<20$ & $<20$ & $<50$ & $<10$ & 340 & $<20$ & $<20$ \\
\hline D30 & $6 / 2 / 1997$ & 400 & 3,230 & $<0.3$ & $<0.1$ & $<0.02$ & $<0.02$ & 0.09 & $<0.2$ & $<60$ & $<60$ & 80 & $<10$ & 270 & $<60$ & $<60$ \\
\hline D30 & $8 / 25 / 1997$ & 410 & 3,370 & $<0.3$ & $<0.1$ & $<0.02$ & 0.06 & 0.0 & $<0.2$ & 130 & $<60$ & 1,790 & $<10$ & 330 & $<60$ & $<60$ \\
\hline D30 & $11 / 19 / 1997$ & 428 & 3,040 & 0.6 & $<0.1$ & $<0.02$ & $<0.02$ & 0.09 & $<0.2$ & $<20$ & $<20$ & 290 & $<10$ & 280 & $<20$ & $<20$ \\
\hline D30 & $1 / 6 / 1998$ & 408 & 3,410 & $<0.3$ & $<0.1$ & $<0.02$ & $<0.02$ & 0.06 & 0.1 & $<20$ & $<20$ & $<50$ & $<10$ & 280 & 20 & $<20$ \\
\hline D30 & 4/13/1998 & 456 & 3,340 & 0.4 & $<0.1$ & $<0.02$ & 0.03 & 0.04 & $<0.2$ & 140 & $<40$ & $<100$ & $<10$ & 350 & $<40$ & $<40$ \\
\hline D30 & $7 / 15 / 1998$ & 370 & 3,030 & 0.6 & $<0.1$ & $<0.02$ & $<0.02$ & 0.05 & $<0.2$ & 80 & $<40$ & 120 & $<10$ & 240 & $<40$ & $<40$ \\
\hline D31 & 9/20/1995 & 734 & 5,900 & $<0.3$ & $<0.1$ & 0.03 & 1.54 & 0.05 & $<0.2$ & $<100$ & $<100$ & $<250$ & $<10$ & 460 & $<100$ & $<100$ \\
\hline $\mathrm{D} 31$ & $12 / 15 / 1995$ & 808 & 6,300 & 2.3 & $<0.1$ & $<0.02$ & 3.59 & $<0.02$ & $<0.2$ & $<20$ & $<20$ & $<50$ & $<10$ & 200 & 30 & $<20$ \\
\hline $\mathrm{D} 31$ & $3 / 5 / 1996$ & 796 & 5,990 & 0.5 & $<0.1$ & 0.03 & 3.17 & 0.03 & $<0.2$ & $<100$ & $<100$ & $<250$ & $<10$ & 210 & $<100$ & $<100$ \\
\hline D31 & $6 / 12 / 1996$ & 1,230 & 6,480 & $<0.3$ & $<0.1$ & $<0.02$ & 1.28 & $<0.02$ & $<0.2$ & $<100$ & $<100$ & $<250$ & $<10$ & 190 & $<100$ & $<100$ \\
\hline D31 & $8 / 21 / 1996$ & 765 & 5,380 & 14.1 & $<0.1$ & $<0.02$ & 0.35 & 0.06 & 0.1 & $<40$ & $<40$ & $<100$ & $<10$ & 710 & 20 & $<40$ \\
\hline D31 & $11 / 7 / 1996$ & 841 & 5,830 & 0.7 & $<0.1$ & $<0.02$ & 0.40 & 0.05 & $<0.2$ & $<20$ & $<20$ & $<50$ & $<10$ & 1,250 & $<20$ & $<20$ \\
\hline $\mathrm{D} 31$ & $2 / 25 / 1997$ & 777 & 5,740 & 0.5 & $<0.1$ & $<0.02$ & 0.44 & $<0.02$ & $<0.2$ & $<20$ & $<20$ & $<50$ & $<10$ & $<20$ & $<20$ & $<20$ \\
\hline D31 & $6 / 3 / 1997$ & 687 & 5,460 & $<0.3$ & $<0.1$ & $<0.02$ & 0.57 & $<0.02$ & $<0.2$ & 220 & $<60$ & $<150$ & $<10$ & 320 & 30 & 40 \\
\hline D31 & $8 / 27 / 1997$ & 767 & 5,190 & 0.7 & $<0.1$ & $<0.02$ & 0.07 & $<0.02$ & $<0.2$ & 110 & $<100$ & $<250$ & $<10$ & 210 & $<100$ & $<100$ \\
\hline
\end{tabular}


Table 5. Water-quality data for samples collected near Deer Trail, Colorado, 1993 through 1998.—Continued

[All data except field properties are for filtered samples analyzed at the Metro Wastewater Reclamation District laboratory; detailed well information is listed in appendix 1; associated quality-control data are included in appendixes 2-8; Temp., temperature; ${ }^{\circ} \mathrm{C}$, degrees Celsius; W.L. ft bmp, depth to groundwater in feet below measuring point; $\mathrm{mg} / \mathrm{L}$, milligrams per liter; $\mu \mathrm{S} / \mathrm{cm}$, microsiemens per centimeter at 25 degrees Celsius; $\mathrm{CaCO}_{3}$, calcium carbonate; $\mathrm{N}$, nitrogen; $\mathrm{P}$, phosphorus; $\mu \mathrm{g} / \mathrm{L}$, micrograms per liter; GW, groundwater; --, no data; <, less than; C1, first composite; c, calculated from calibration data; PW, pore water; ft, feet]

\begin{tabular}{|c|c|c|c|c|c|c|c|c|c|c|c|c|c|c|c|}
\hline $\begin{array}{l}\text { Sample } \\
\text { site } \\
\text { (fig. 2; } \\
\text { table 2) }\end{array}$ & $\begin{array}{c}\text { Sample } \\
\text { date }\end{array}$ & $\begin{array}{l}\text { Sample } \\
\text { type }\end{array}$ & $\begin{array}{l}\text { Temp., } \\
\text { air, } \\
\text { field, } \\
{ }^{\circ} \mathrm{C}\end{array}$ & $\begin{array}{l}\text { W.L. ft } \\
\text { bmp }\end{array}$ & $\begin{array}{c}\text { Dissolved } \\
\text { oxygen, } \\
\text { field, } \\
\text { mg/L }\end{array}$ & $\begin{array}{c}\text { pH, field, } \\
\text { standard } \\
\text { units }\end{array}$ & $\begin{array}{c}\text { Specific } \\
\text { conduc- } \\
\text { tance, } \\
\text { field, } \\
\mu S / c m\end{array}$ & $\begin{array}{l}\text { Temp., } \\
\text { water, } \\
\text { field, } \\
{ }^{\circ} \mathrm{C}\end{array}$ & $\begin{array}{c}\text { Alka- } \\
\text { linity, } \\
\text { lab, } \\
\mathrm{mg} / \mathrm{L} \text { as } \\
\mathrm{CaCO}_{3}\end{array}$ & $\begin{array}{c}\text { Hard- } \\
\text { ness, } \\
\mathrm{mg} / \mathrm{L} \text { as } \\
\mathrm{CaCO}_{3}\end{array}$ & $\begin{array}{c}\text { Dis- } \\
\text { solved } \\
\text { solids, } \\
\text { residue } \\
\text { at } 180^{\circ} \mathrm{C} \text {, } \\
\mathrm{mg} / \mathrm{L}\end{array}$ & $\begin{array}{l}\text { Cal- } \\
\text { cium, } \\
\text { mg/L }\end{array}$ & $\begin{array}{l}\text { Chlo- } \\
\text { ride, } \\
\text { mg/L }\end{array}$ & $\begin{array}{c}\text { Potas- } \\
\text { sium, } \\
\text { mg/L }\end{array}$ & $\begin{array}{c}\text { Mag- } \\
\text { ne- } \\
\text { sium, } \\
\text { mg/L }\end{array}$ \\
\hline D32 & $9 / 28 / 1995$ & GW & -- & 27.87 & -- & 7.2 & 3,660 & 16.0 & 278 & 2,580 & 3,720 & 476 & 9 & 2.0 & 236 \\
\hline D32 & $12 / 13 / 1995$ & GW & 10.0 & 27.80 & -- & 7.0 & 3,600 & 14.0 & 276 & 2,310 & 3,680 & 522 & 7 & 2.0 & 244 \\
\hline D32 & 8/20/1996 & GW & 32 & 28.06 & 0.8 & 7.1 & 3,440 & 23.2 & 285 & 2,330 & 3,640 & 530 & 6 & 2.0 & 259 \\
\hline D32 & 8/26/1997 & GW & 29 & 28.28 & 1.2 & 7.2 & 3,470 & 18.9 & 295 & 2,360 & 3,740 & 516 & 6 & $<0.1$ & 270 \\
\hline D32 & $11 / 18 / 1997$ & GW & 12 & 28.01 & 1.4 & 7.1 & 3,680 & 13.6 & 310 & 2,160 & 3,630 & 518 & 6 & $<0.1$ & 260 \\
\hline D32 & $1 / 7 / 1998$ & GW & 4.4 & 27.89 & 1.5 & 7.1 & 3,480 & 14.4 & 290 & 2,360 & 3,670 & 496 & 5 & $<0.1$ & 227 \\
\hline D32 & $7 / 15 / 1998$ & GW & 35 & 27.79 & 1.6 & 7.3 & 3,540 & 17.8 & 317 & 2,370 & 3,730 & 614 & 8 & $<0.1$ & 240 \\
\hline D33 & 9/27/1995 & GW & -- & 14.94 & -- & 7.4 & 3,880 & 14.5 & 232 & 2,420 & 3,940 & 502 & 22 & 14.0 & 331 \\
\hline D33 & $2 / 25 / 1997$ & GW & 14 & 14.80 & 4.8 & 7.3 & 3,840 & 11.3 & 232 & 2,650 & 3,910 & 511 & 15 & 12.0 & 308 \\
\hline D33 & $6 / 5 / 1997$ & GW & 25 & 14.68 & 5.0 & 7.2 & 3,770 & 11.9 & 230 & 2,270 & 4,760 & 443 & 16 & 11.0 & 292 \\
\hline D33 & 8/26/1997 & GW & 29 & 14.25 & 4.5 & 7.4 & 3,770 & 13.4 & 237 & 2,520 & 3,970 & 496 & 14 & 10.0 & 317 \\
\hline D33 & 11/18/1997 & GW & 7 & 14.34 & 4.2 & 7.4 & 3,790 & 12.9 & 243 & 2,030 & 3,940 & 549 & 14 & 16.0 & 335 \\
\hline G1 & $7 / 23 / 1993$ & GW & -- & -- & -- & -- & 1,620 & 14.5 & -- & 912 & -- & 302 & 4 & 3.3 & 47 \\
\hline G1 & $8 / 26 / 1993$ & GW & -- & -- & -- & 7.4 & 1,720 & 14.5 & 150 & 961 & 1,490 & 320 & $<1$ & 3.4 & 27 \\
\hline G2 & 7/23/1993 & GW & -- & -- & -- & -- & 574 & 16.3 & & 231 & -- & 65 & 18 & 1.7 & 19 \\
\hline G2 & $8 / 26 / 1993$ & GW & -- & -- & -- & 7.8 & 830 & 14.6 & 170 & 225 & 535 & 69 & 19 & 2.6 & 20 \\
\hline G3 & 7/23/1993 & GW & -- & -- & -- & -- & 2,170 & 14.5 & & 1,150 & & 303 & 12 & 5.0 & 131 \\
\hline G3 & $8 / 26 / 1993$ & GW & -- & -- & -- & 7.5 & 1,650 & 14.4 & 198 & 888 & 1,350 & 243 & 2 & 4.0 & 76 \\
\hline S1 & $7 / 23 / 1993$ & Stream & -- & -- & -- & -- & 235 & 18.6 & -- & 51 & -- & 16 & -- & 7.4 & 3 \\
\hline S2 & $7 / 23 / 1993$ & Pond & -- & -- & -- & -- & 175 & 23.7 & -- & 77 & -- & 15 & 4 & 3.4 & 7 \\
\hline S3 & $7 / 23 / 1993$ & Pond & -- & -- & -- & -- & 3,710 & 22.7 & -- & 1,630 & -- & 380 & 26 & 17.7 & 220 \\
\hline $\mathrm{V} 2 \mathrm{~L} 7^{1}$ & $4 / 22 / 1994$ & $\begin{array}{c}\text { PW depth } \\
=3 \mathrm{ft}\end{array}$ & -- & -- & -- & -- & -- & -- & -- & -- & -- & 105 & 140 & 9 & $<5$ \\
\hline V2L8 ${ }^{1}$ & $4 / 22 / 1994$ & $\begin{array}{c}\text { PW depth } \\
=1.5 \mathrm{ft}\end{array}$ & -- & -- & -- & -- & -- & -- & -- & -- & -- & 67 & 33 & 12 & $<11$ \\
\hline V3L $3^{1}$ & $4 / 21 / 1994$ & $\begin{array}{c}\text { PW depth } \\
=1.5 \mathrm{ft}\end{array}$ & -- & -- & -- & -- & -- & -- & -- & -- & -- & 200 & 1,200 & 60 & $<200$ \\
\hline V3L8 ${ }^{1}$ & $4 / 22 / 1994$ & $\begin{array}{c}\text { PW depth } \\
=1.5 \mathrm{ft}\end{array}$ & -- & -- & -- & -- & -- & -- & -- & -- & -- & 90 & 110 & 7 & $<10$ \\
\hline
\end{tabular}


Table 5. Water-quality data for samples collected near Deer Trail, Colorado, 1993 through 1998.—Continued

[All data except field properties are for filtered samples analyzed at the Metro Wastewater Reclamation District laboratory; detailed well information is listed in appendix 1; associated quality-control data are included in appendixes 2-8; Temp., temperature; ${ }^{\circ} \mathrm{C}$, degrees Celsius; W.L. $\mathrm{ft}$ bmp, depth to groundwater in feet below measuring point; $\mathrm{mg} / \mathrm{L}$, milligrams per liter; $\mu \mathrm{S} / \mathrm{cm}$, microsiemens per centimeter at 25 degrees Celsius; $\mathrm{CaCO}_{3}$, calcium carbonate; $\mathrm{N}$, nitrogen; $\mathrm{P}$, phosphorus; $\mu \mathrm{g} / \mathrm{L}$, micrograms per liter; GW, groundwater; --, no data; <, less than; C1, first composite; c, calculated from calibration data; PW, pore water; ft, feet]

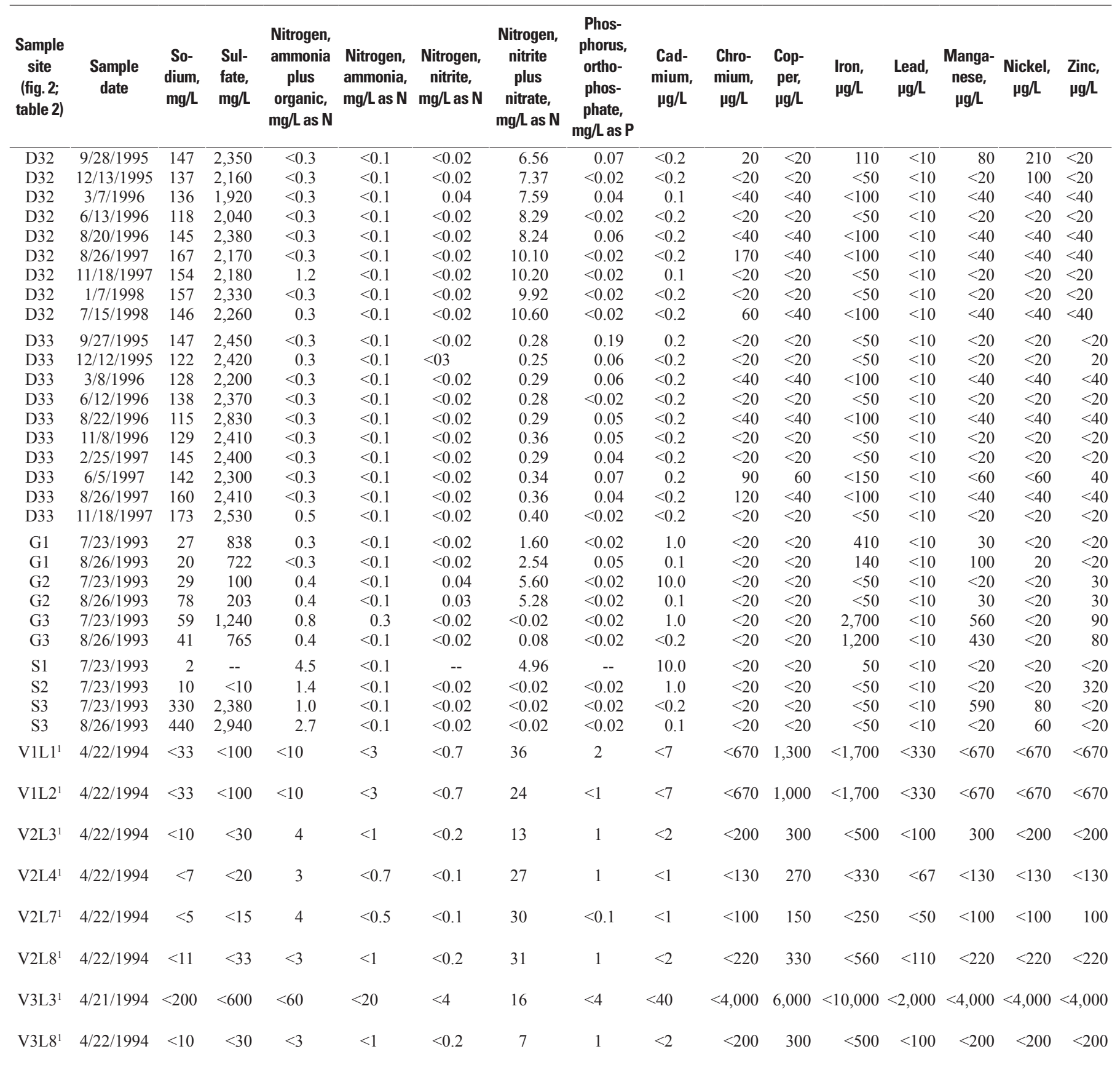

${ }^{1} \mathrm{VX}$ refers to unsaturated-zone plot number (fig. 2); LX refers to lysimeter number. 
Table 6. Bacteria data for water-quality samples collected near Deer Trail, Colorado, 1993 through 1998.

[All samples cultured by the Metro Wastewater Reclamation District Laboratory on the same day sampled; pond samples were a single grab sample from near the bank; GW, groundwater; SW, pond; QC, quality control; $\mathrm{mL}$, milliliters; <, less than; >, greater than]

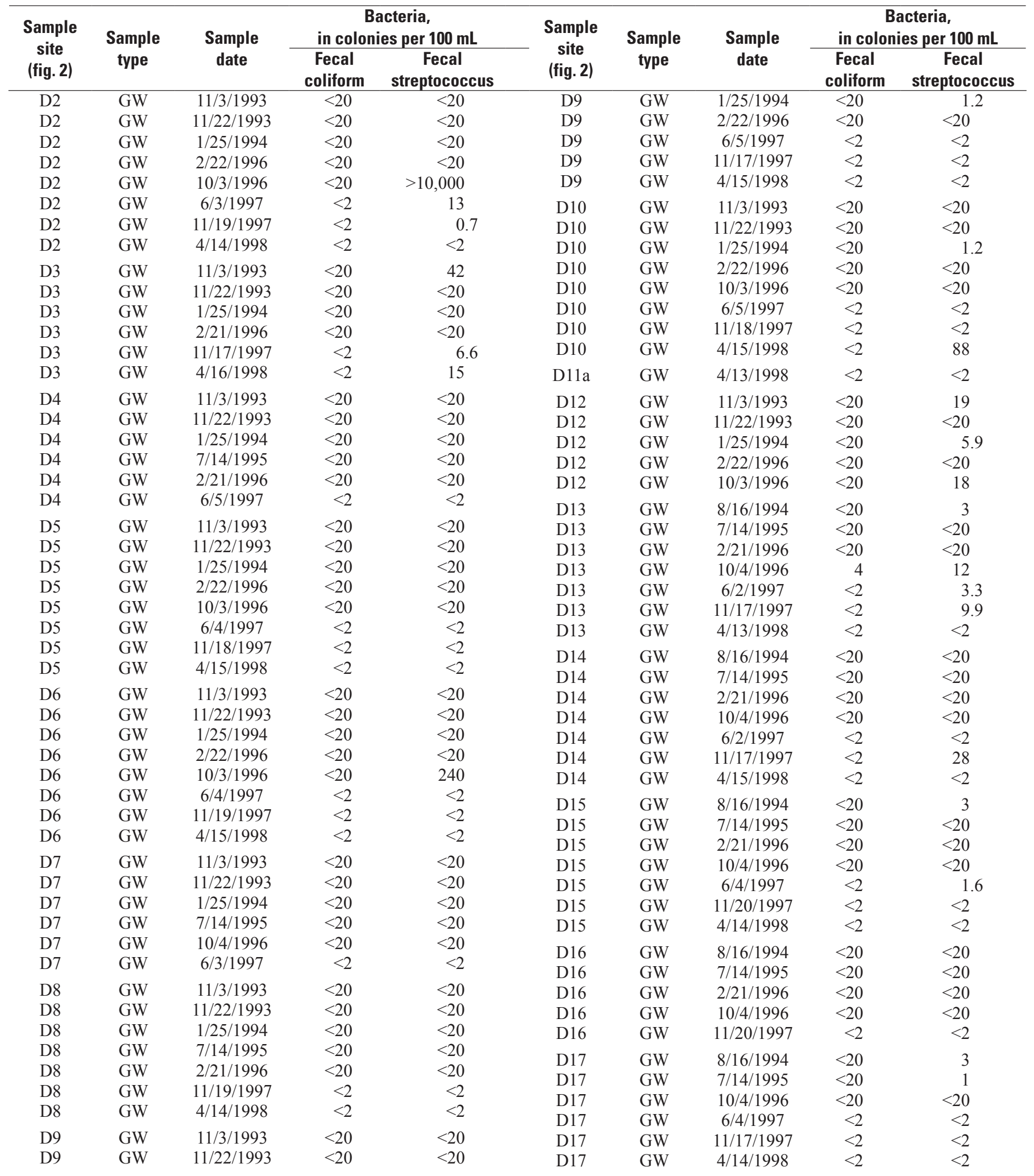


Table 6. Bacteria data for water-quality samples collected near Deer Trail, Colorado, 1993 through 1998.—Continued

[All samples cultured by the Metro Wastewater Reclamation District Laboratory on the same day sampled; pond samples were a single grab sample from near the bank; GW, groundwater; SW, pond; QC, quality control; $\mathrm{mL}$, milliliters; <, less than; >, greater than]

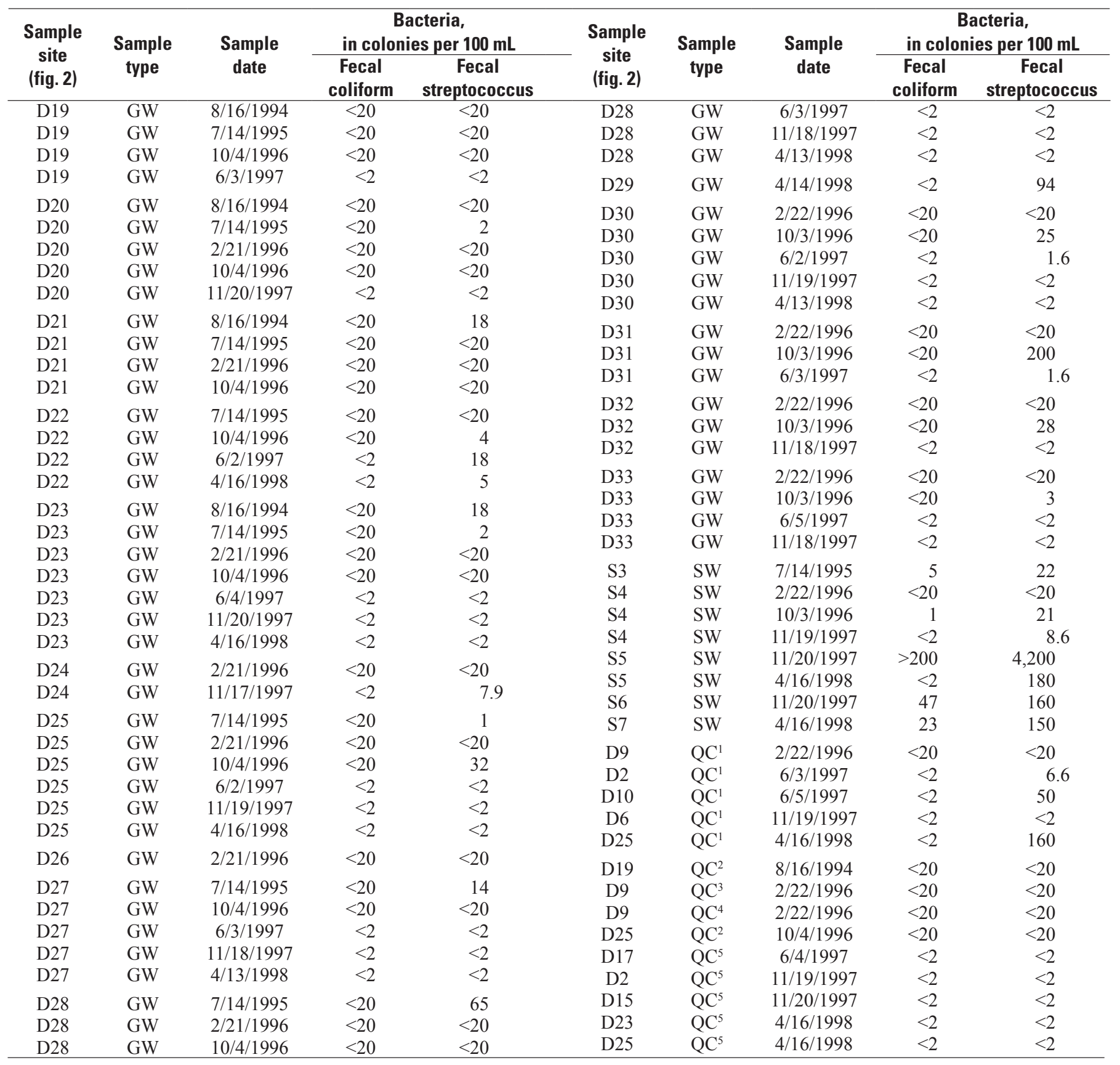

${ }^{1}$ Replicate groundwater sample.

${ }^{2}$ Field blank at a groundwater site; deionized water through sampling bailer.

${ }^{3}$ Field blank at a groundwater site; municipal tap water, no sampling equipment.

${ }^{4}$ Field blank at a groundwater site; municipal tap water through sampling bailer.

${ }^{5}$ Field blank at a groundwater site; sterile, buffered water through sampling bailer. 
Table 7. Water-quality data for redox-indicator sampling near Deer Trail, Colorado, July 1999.

[All data except field properties from filtered samples (unless otherwise noted) and from U.S. Geological Survey laboratory; temp., temperature; ${ }^{\circ} \mathrm{C}$, degrees Celcius; DO, dissolved oxygen; $\mathrm{mg} / \mathrm{L}$, milligrams per liter; lab, laboratory; $\mu \mathrm{S} / \mathrm{cm}$, microsiemens per centimeter at $25^{\circ} \mathrm{C}$; ANC, acid neutralizing capacity; $\mathrm{CaCO}_{3}$, calcium carbonate; $\%$, percent; $\mu \mathrm{g} / \mathrm{L}$, micrograms per liter; --, no data; $<$, less than; E, estimated by laboratory]

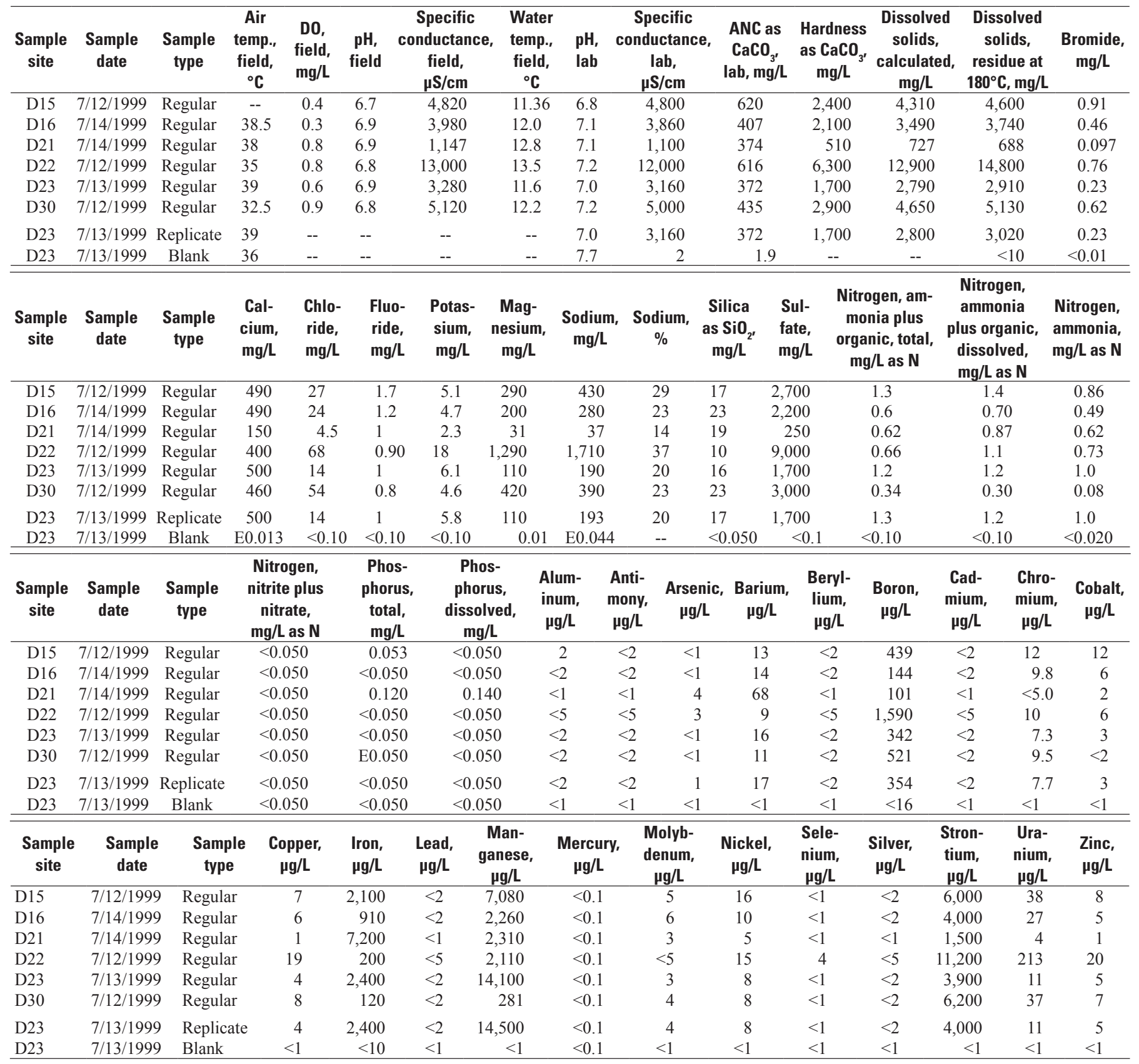




\section{Baseline Water Quality}

For this study, baseline data were collected from the study area after the area had been farmed and inhabited but before biosolids were applied. The baseline data do not represent pristine natural conditions but likely are affected by anthropogenic activities such as agriculture, roads, and habitation. All baseline data from the study area are included in tables 5 and 6 (the 1993 data for wells D2 through D12, G1 through G3, and for surface-water sites S1, S2, and S3). These baseline data were collected during a brief time period and so do not account for variability due to seasonal natural processes. There was not sufficient time to collect seasonally representative samples from each well or pond before biosolids were applied. Baseline concentrations could have been smaller or larger at other times of the year at these sites. These baseline data also were not collected using the strict sampling methods that were used for collecting water-quality data after 1995 and so should not be statistically compared to later data.

Baseline data (considered "background data" by the Colorado Department of Public Health and Environment [1997]) indicate water-quality information for the study area in the approximately 6 months before biosolids applications to the study area began. Baseline water quality in the study area was marginal at some sites and better at other sites. Large concentrations of chloride, sulfate, nitrate, iron, and manganese were detected in some baseline samples (table 5). A summary of selected baseline data is shown in figure 8. Sulfate concentrations in baseline samples generally were greater than $1,000 \mathrm{mg} / \mathrm{L}$ (fig. 8). The largest concentration of sulfate in baseline samples was at well D6 where sulfate concentration was greater than $10,000 \mathrm{mg} / \mathrm{L}$. Nitrogen concentrations in the baseline samples generally were in the oxidized form (nitrate). About one-half of the sites sampled for baseline water quality had nitrate concentrations of 1 milligram per liter as nitrogen (mg-N/L) or greater. Five sites (wells D5, D6, D9, and G2, and stream-site $\mathrm{S} 1$ ) had nitrate concentrations greater than $3 \mathrm{mg}-\mathrm{N} / \mathrm{L}$ in baseline samples. Three sites (wells D5 and G2 and stream-site S1) had nitrate concentrations in the range of about 5-10 mg-N/L in baseline samples (fig. 8). One site (well D9) had nitrate concentrations greater than $10 \mathrm{mg}-\mathrm{N} / \mathrm{L}$ in baseline samples before biosolids applications began (fig. 8). Iron concentration in baseline samples was less than $50 \mu \mathrm{g} / \mathrm{L}$ at most sites but was in the range of $50-500 \mu \mathrm{g} / \mathrm{L}$ at two sites (G1 and $\mathrm{S} 1)$ and greater than $1,000 \mu \mathrm{g} / \mathrm{L}$ at one site (G3) (fig. 8). Manganese concentration in baseline samples was in the range of $101-500 \mu \mathrm{g} / \mathrm{L}$ at one site (well D12) and greater than $500 \mu \mathrm{g} / \mathrm{L}$ at six sites (wells D3, D6, D7, D8, and G3, and pond-site S3) (fig. 8). The largest manganese concentrations measured in baseline samples were at wells D6 $(7,400 \mu \mathrm{g} / \mathrm{L})$ and D8 $(6,900 \mu \mathrm{g} / \mathrm{L})$.

In addition to the baseline data shown in figure 8, many other water-quality constituents were detected in baseline samples from the study area (table 5). Chloride was detected in nearly all baseline samples; the maximum chloride concentration in baseline samples was $373 \mathrm{mg} / \mathrm{L}$ from well D6. Small concentrations of phosphorus (orthophosphate as phosphorus) were detected in baseline samples from all the monitoring wells ("D" numbered wells) but not in all the other baseline water samples ("G" and " $\mathrm{S}$ " sites); the largest concentration of orthophosphate as phosphorus in a baseline sample was from well D4 (0.29 mg/L as phosphorus). Cadmium was detected in most baseline samples; the maximum concentration in samples from the monitoring wells was $3.8 \mu \mathrm{g} / \mathrm{L}$ from well D6, but the maximum concentration detected in any baseline sample was $10 \mu \mathrm{g} / \mathrm{L}$ from sites G2 and S1. Chromium and copper were not detected in any baseline samples, but nickel and zinc were detected in baseline samples. The maximum nickel concentration in baseline samples was from well D6 $(200 \mu \mathrm{g} / \mathrm{L})$. The maximum zinc concentration in baseline samples was from well D12 $(110 \mu \mathrm{g} / \mathrm{L})$. Bacteria (fecal streptococcus) were found in baseline samples from wells D3 and D12, and counts in the D3 sample were about twice the MRL. The baseline data indicate that major-ion, nutrient, trace-element, and bacteria sources other than biosolids were present in the study area and that water in the study area was of variable quality before biosolids ever were applied.

\section{Variability in Water Quality}

Variability in water quality of the study area was evident from the baseline data as well as from the data collected after biosolids applications began. Therefore, this discussion of variability in water quality includes all data collected during 1993-1998; effects from biosolids applications will be discussed in a later section, Biosolids Effects. Water quality varied spatially, both across the study area and with depth of water sampled. Water quality also varied temporally during 1993-1998.

The hydrology of the study area was described by Yager and Arnold (2003) and likely accounts for some of the variability in water quality. Precipitation caused runoff from farmed fields, caused intermittent and ephemeral streams to flow, and fed natural and anthropogenic ponds. During dry periods, pond water evaporated and desiccation cracks formed. Some ponds were fed by groundwater discharge and some ponds recharged groundwater. Groundwater also recharged preferentially through desiccation cracks and coarse-grained deposits and outcrops, not uniformly through the unsaturated zone beneath all fields. Ponds were present in various locations of the study area at different times, but a few ponds were consistently present such as near wells D2, D4, D8, D20, D22, D23, and D30. Such ponds likely were maintained by groundwater discharge but also could provide recharge to groundwater when the water table dropped. Detention structures across stream valleys that were created to decrease erosion resulted in new (anthropogenic) ponds (such as those in 1996 or 1997 near wells D12, D24, D25, D26, and D32) or increased the duration and size of natural ponds. Natural ponds 


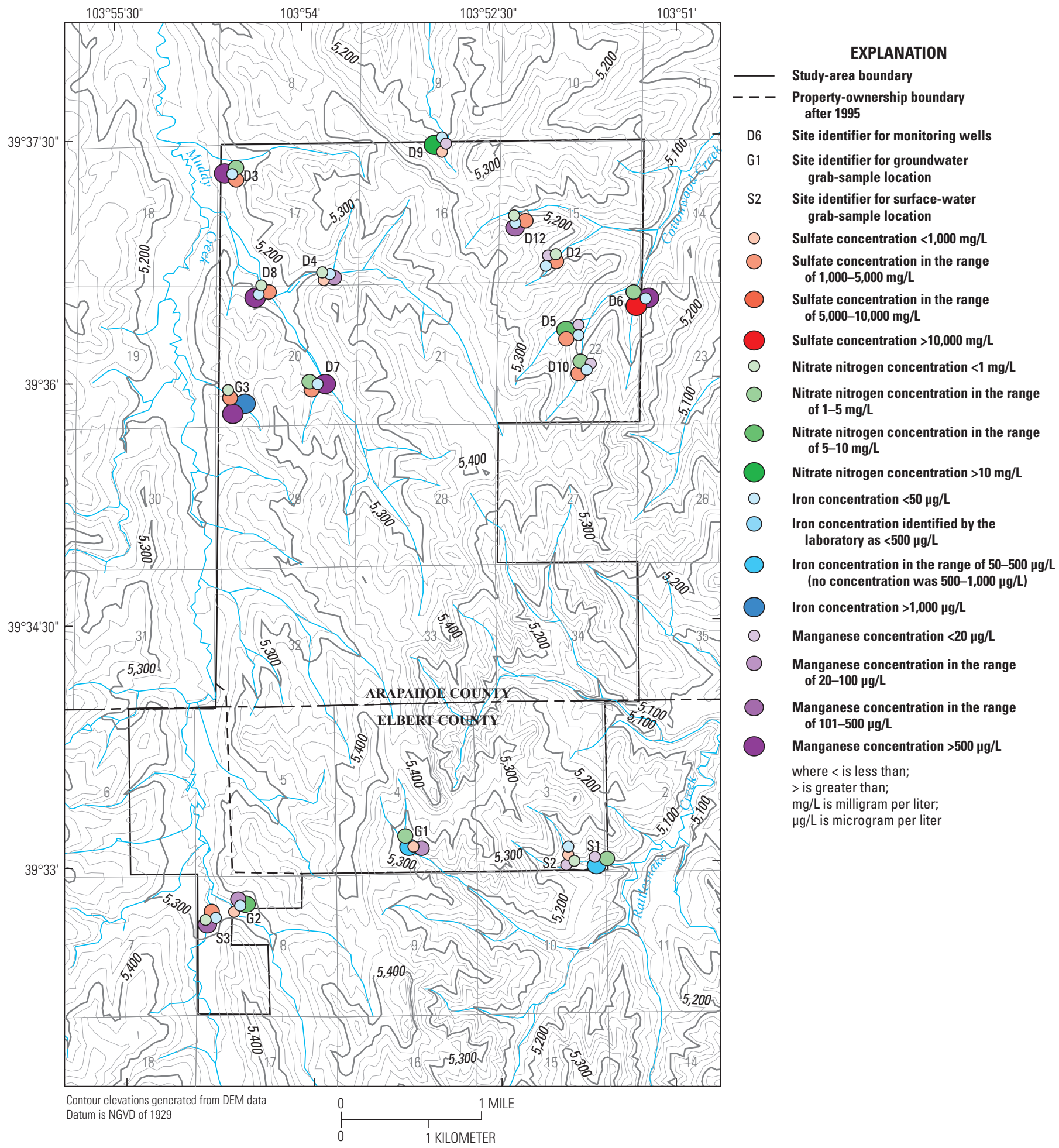

Figure 8. Baseline water quality for selected constituents in groundwater and surface-water samples collected in 1993 at the Metro Wastewater Reclamation District property. [Biosolids applications began in late 1993 to early 1994 after these samples were collected] 
occasionally formed near wells D6, D9, D14, D15, D16, D19, D28, and D31 but were transient. Such ponds likely provided recharge to groundwater until they dried out. Ponds captured runoff from nearby fields that could be affected by farming, biosolids applications, or animal waste. Subsequent evaporation concentrated many constituents in pond water as can be seen by the differences in the July and August 1993 concentrations in water samples from pond $\mathrm{S} 3$ (table 5). These processes naturally increased water-quality variability in the study area.

\section{Spatial Variability}

Water quality varied from site to site throughout the study area during 1993-1998 (tables 5-7). Spatial variability in the baseline water quality of the study area is evident from the different distributions of concentration data at the different sites shown in figure 8 . Spatial variability in the water-quality data collected after 1993 also is evident (tables 5-7). Concentrations of major ions, nutrients, trace elements, and bacteria varied from site to site in the study area. Concentrations of sulfate, nitrate, iron, and manganese varied the most. Drainage basins can receive similar rain and snow amounts, have a similar topographic and hydraulic gradient, receive similar runoff amounts and quality, and have other similar characteristics that can affect water quality, but water quality generally was not similar within the four drainage basins of the study area. Muddy Creek generally had similar water quality along the Muddy Creek main stem drainage valley (including wells D15 and D21), but had dissimilar water quality in tributary drainage valleys (for example, wells D13, D17, D20, and D25). Cottonwood Creek drainage basin had similar concentration patterns for some constituents in some locations (nitrate concentrations generally increased at wells D5, D6, and D32), but generally dissimilar water quality (compare constituent concentrations at well D6 with other wells in this drainage basin). Although well D9 was the only monitoring well in the Badger Creek drainage basin, the pattern of nitrate concentrations at well D9 was most similar to that of well D10 (Cottonwood Creek drainage basin). Although well D22 was the only monitoring well in Rattlesnake Creek drainage basin, sulfate concentrations (and some other constituents) were more similar to well D6 (Cottonwood Creek drainage basin) concentrations than to those of other wells. Therefore, water quality did not appear to vary according to drainage basin in the study area.

Major-ion concentrations generally were higher in groundwater than in surface water but varied considerably. Median chloride concentration ranged from less than $1 \mathrm{mg} / \mathrm{L}$ in samples from well D11a to $394.5 \mathrm{mg} / \mathrm{L}$ in samples from well D6. Median sulfate concentration ranged from $34 \mathrm{mg} / \mathrm{L}$ in samples from well D11a to $12,200 \mathrm{mg} / \mathrm{L}$ in samples from well D6. Major ion data can be used to describe water composition. In general, groundwater of the study area was calciummagnesium-sulfate type or calcium-bicarbonate type (fig. 9). Sometimes a diagram of major-ion concentrations (like the trilinear diagrams shown in figure 9) indicates that water composition is similar by geographic area, by aquifer type (bedrock or alluvial), by geology (sandstone or shale), or by treatment (biosolids or no biosolids). Trilinear diagrams of major-ion data for the study area did not indicate much difference in water composition by drainage basin, treatment, site type, or well depth (fig. 9). However, the trilinear diagram indicates that water-composition groupings (D in fig. 9) align somewhat with the geologic map of the Limon Quadrangle (Sharps, 1980) as demonstrated in figure 10. Discrepancies between data groupings and the formation delineations from Sharps (1980) could be a result of minor inaccuracies in geologic mapping (such as from scale changes when the large geologic map was magnified to fit the small study area, from interpolation during mapping, or from later erosion of the landscape), or lithologic differences within formations that result in localized mineralogical or geochemical variations. Little detailed lithological, mineralogical, or core-chemical information is available for the wells in the study area to verify that water composition in the study area largely is controlled by geology. However, information obtained from coring the monitoring wells for the USGS expanded monitoring program (the "DTX" numbered wells in fig. 3; Stevens and others, 2003) supports the hypothesis that major-ion concentrations in groundwater varied more by lithology or geologic formation than by drainage basin, biosolids treatment, site type, or well depth.

Nitrate concentration varied considerably across the study area (table 5; fig. 8). At individual monitoring wells, median nitrate concentration ranged from less than $0.02 \mathrm{mg}-\mathrm{N} / \mathrm{L}$ in samples from many wells to $24.05 \mathrm{mg}-\mathrm{N} / \mathrm{L}$ in samples from well D24. Nitrate concentrations were less than $2 \mathrm{mg}-\mathrm{N} / \mathrm{L}$ in samples from both deep bedrock-aquifer wells (D11a and D29), but concentrations varied greatly in samples from shallow wells. Nitrate concentrations generally were largest in samples from shallow bedrock-aquifer wells in the north part of the study area (wells D9 and D24). Nitrate concentrations generally were small in samples from wells near wetland areas such as those along Muddy Creek (wells D15, D16, D20, D21, and D23; table 5) and in samples from wells along lower Cottonwood Creek (wells D2, D12, D30, and D31; table 5). Nitrate or ammonia plus organic nitrogen (sometimes called Kjeldahl nitrogen) was the predominant nitrogen compound in samples from these sites. Nitrate concentrations in samples from upper Cottonwood Creek wells (D5, D6, D10, D32, and D33; table 5) generally were higher than in samples from lower Cottonwood Creek wells (wells D2, D12, D30, and D31).

Trace-element concentrations varied considerably across the study area for a few constituents (table 5; fig. 8). At individual monitoring wells, median iron concentration ranged from less than $50 \mu \mathrm{g} / \mathrm{L}$ in samples from many wells to $6,850 \mu \mathrm{g} / \mathrm{L}$ in the samples from well D29. Median manganese concentration ranged from less than $20 \mu \mathrm{g} / \mathrm{L}$ in samples from many wells to $16,000 \mu \mathrm{g} / \mathrm{L}$ in the samples from well D23. Concentrations of dissolved iron and manganese generally were higher in wetland-area wells, such as along Muddy Creek. Median concentrations of cadmium, chromium, copper, nickel, lead, and zinc varied little from site to site and generally were less than the MRL in samples from all sites. 

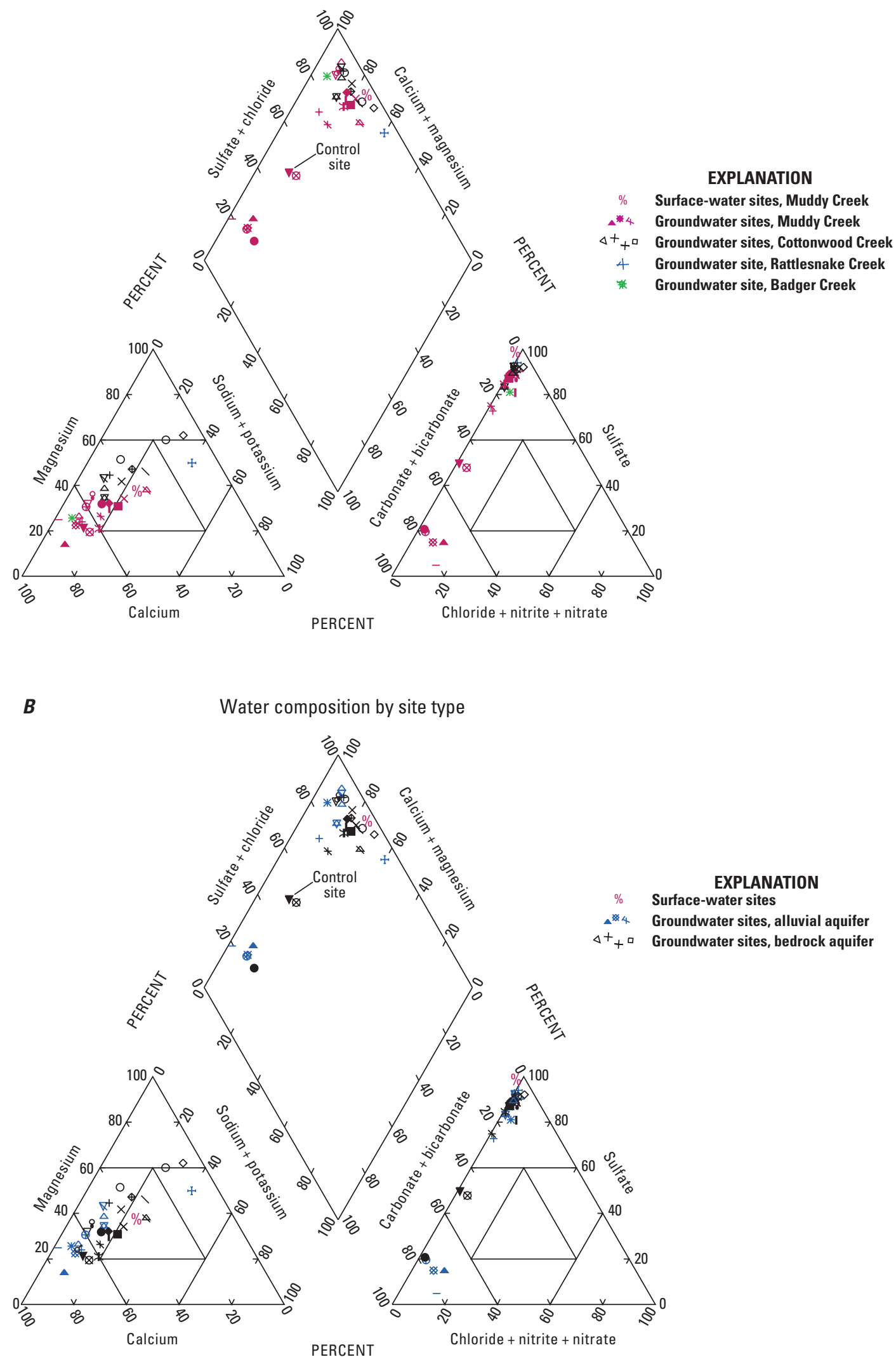

Figure 9. Trilinear diagrams showing water type for $(A)$ drainage basin, $(B)$ site type, $(C)$ well depth, and (D) water-composition groupings near Deer Trail, Colorado, based on median data for 1993-1998. 


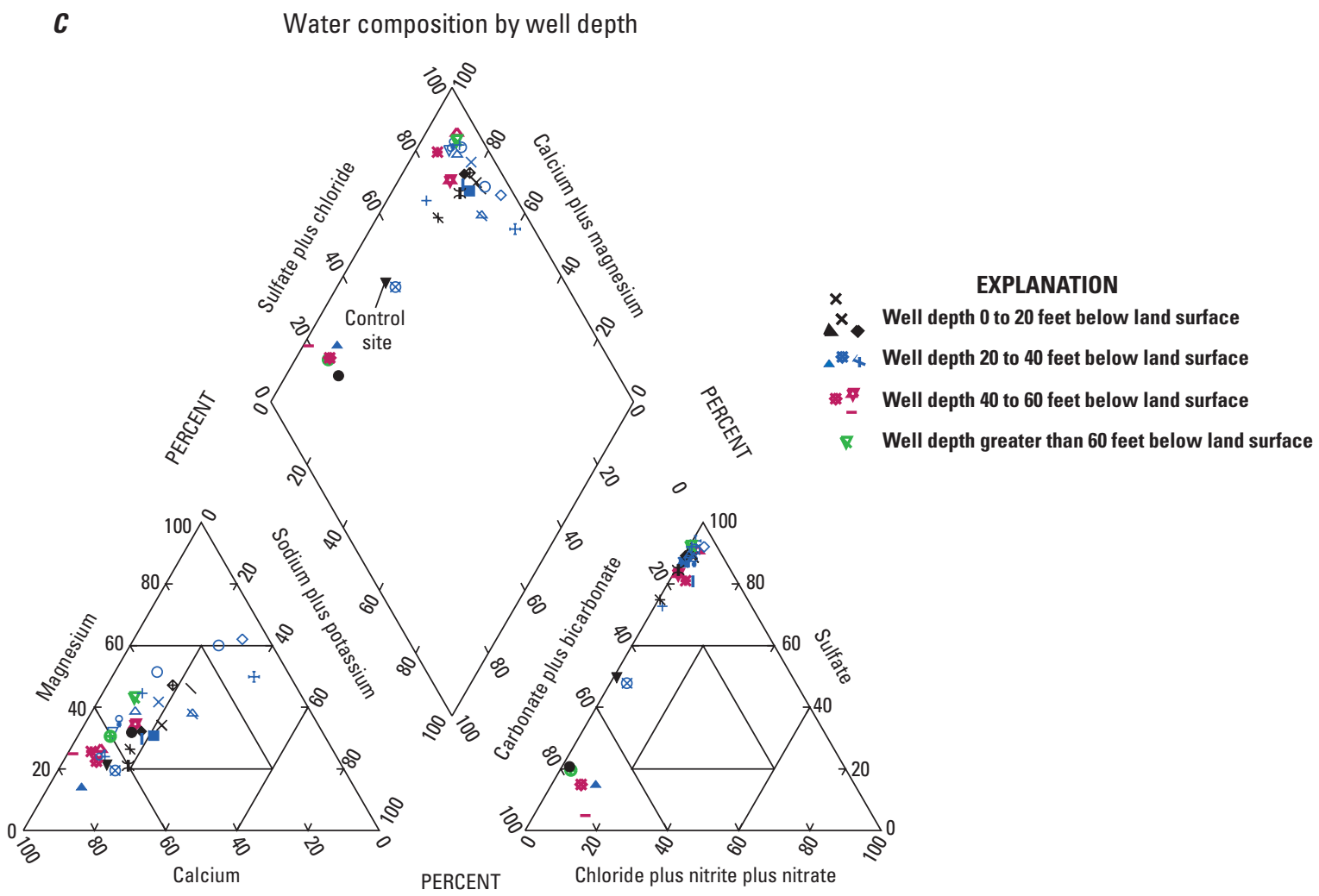

D

Water composition groupings

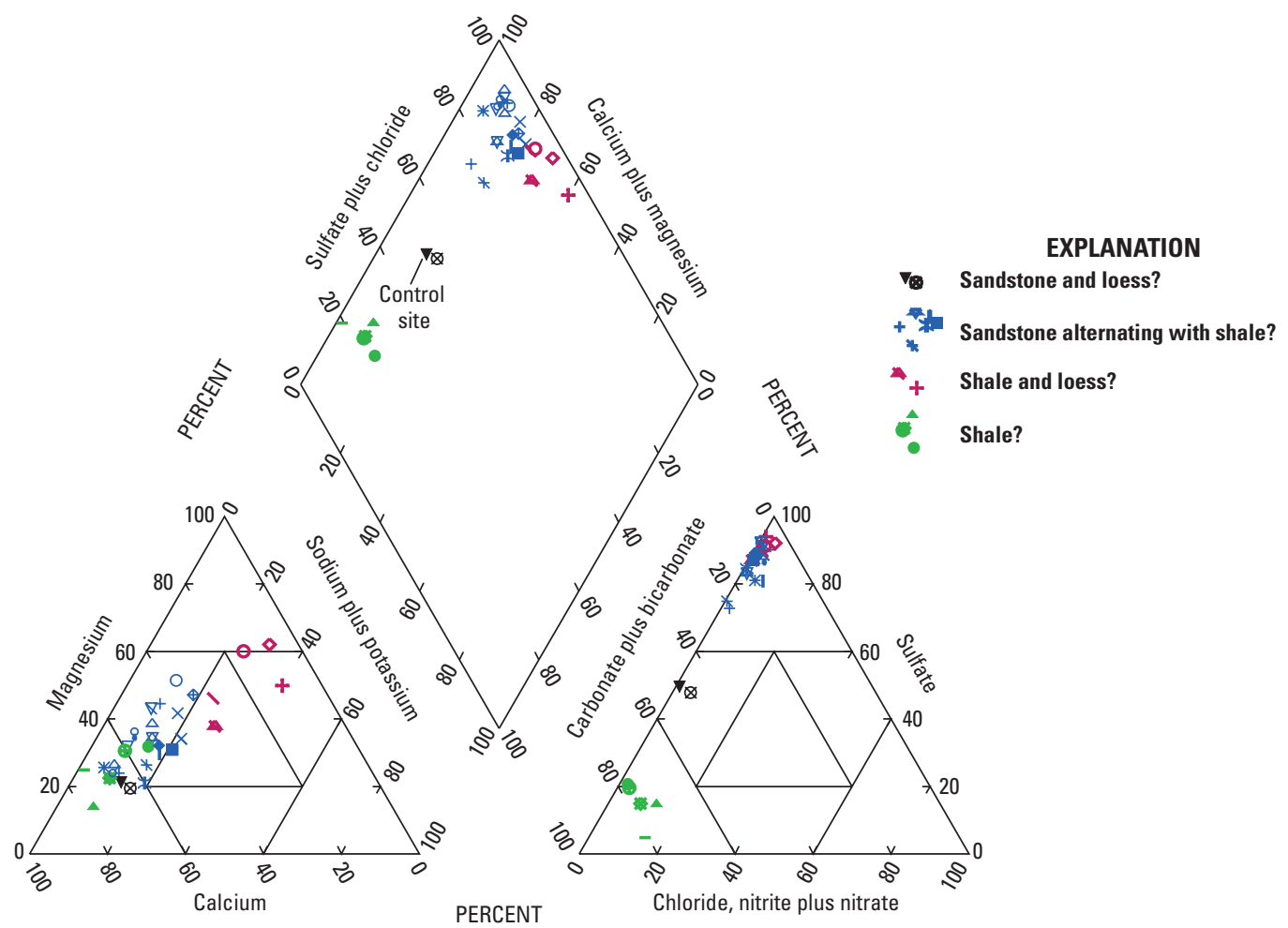

Figure 9. Trilinear diagrams showing water type for $(A)$ drainage basin, $(B)$ site type, $(C)$ well depth, and (D) water-composition groupings near Deer Trail, Colorado, based on median data for 1993-1998.-Continued 


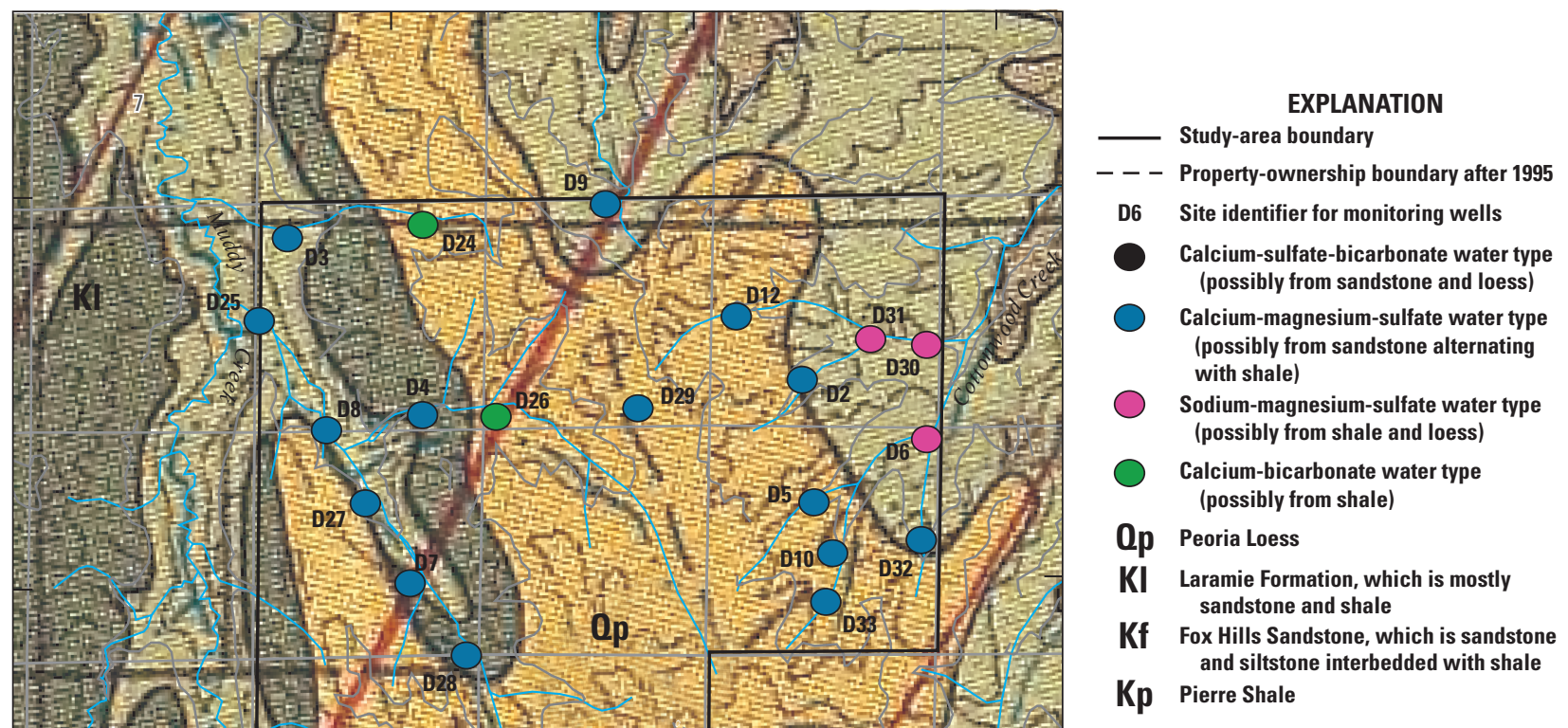

Contour elevations generated from DEM data Datum is NGVD of 1929

Geologic map base from Sharps (1980)

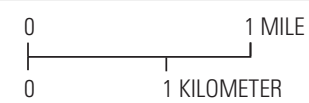

Figure 10. Groundwater major-ion water-composition groups (figure 9D) overlaying the geologic map presented by Sharps (1980). 
Bacteria concentrations varied across the study area; however, no spatial patterns in fecal coliform and fecal streptococcus bacteria counts were observed for 1993-1998 in the study area (table 6). Although these bacteria were detected in samples collected from every drainage basin, fecal coliform and fecal streptococcus bacteria were not detected in most of the samples from the study area. Fecal streptococcus bacteria were detected more frequently than fecal coliform bacteria. In general, fecal coliform and fecal streptococcus bacteria were not routinely detected in samples from the same site. No fecal coliform or fecal streptococcus bacteria were detected in any samples from wells D4, D5, D7, D8, D16, D19, or D26. Of the eight surface-water samples collected, seven had detections of these bacteria. Pond samples always had larger bacteria counts than samples collected at the same time from nearby wells, except at well D30 and the nearby pond (S4) where bacteria counts were similar (table 6). The erratic and wide-ranging bacteria counts in the samples from the study area caused the USGS to substantially revise the field sampling method in early 1997 to minimize any possible effect from field procedures or equipment. Therefore, the USGS has more confidence in the bacteria data from 1997 and 1998 because field equipment and field methods were not contributing bias or variability. Laboratory processes likely contributed some bias and variability in the 1993-1998 bacteria data; note the variation in data for concurrent replicate pairs in 1997 and 1998.

Water-quality concentration did not correlate well with depth of sample although some generalizations can be made for some constituents. Water-quality data for chloride, sulfate, nitrate, iron, and manganese are plotted with sample depth and (for groundwater samples) depth to groundwater in figure 11. Groundwater was sampled from about $10 \mathrm{ft}$ to about $150 \mathrm{ft}$ below land surface, pore water was sampled from 1.5 - and $3-\mathrm{ft}$ depths, and surface water always was sampled at land surface (0-ft depth). Depth to groundwater below land surface ranged from a few feet at well D23 to about $150 \mathrm{ft}$ at well D29. Most of the variations in concentration shown in figure 11 result from concentration variability at the same site. For example, chloride, sulfate, and manganese concentrations in samples from well D6 were large and fluctuated more than those from most other wells; iron concentrations in samples from well D21 were large and fluctuated more than those from other wells. In general, smaller chloride, nitrate, and manganese concentrations were characteristic of deeper wells; however, not all shallower samples or wells had higher concentrations, and few deeper wells were included in this study. The largest nitrate concentration detected in the study-area water was in the pore water at 1.5 -ft depth (table 5). The unsaturated-zone data (April 1994) indicate that nitrate concentrations in the upper $3 \mathrm{ft}$ of soil pore water can be as high or higher than groundwater concentrations (table 5).

Depth affected pore-water availability for sampling, as well as water quality. Samples of pore water collected from the unsaturated zone in upland areas of the study area were used to indicate whether a plume of contaminants was migrating from near the land surface downwards through the unsaturated zone and eventually into the shallow groundwater. Of the
36 lysimeters installed (four different plots), only 8 samples could be obtained from 3 plots in April 1994. Only some (not all) of the shallowest lysimeters (installed 1.5-3 ft below land surface) yielded pore-water samples. Although the spring of 1994 was wet, no samples could be obtained from the control plot because all the lysimeters were dry. No samples could be obtained from the same lysimeters from any depth the following spring (1995) despite the unusually wet weather that preceded the sampling effort. These pore-water data (and lack of samples) indicate preferential recharge patterns, not diffuse recharge through all the agricultural fields in the study area. More pore water was available for sampling in the $1.5-\mathrm{ft}$ depth (yielded 7 samples) than in the 3 -ft depth (yielded 1 sample) or the 5 -ft depth (yielded 0 samples). When constituents were detected, the single pore-water sample obtained from the 3-ft depth (V3L7) was not the maximum or the minimum concentration, so little effect of depth on pore-water concentration can be surmised. The data indicate that nitrogen in the pore water was primarily in the form of nitrate (table 5). Chloride, nitrate, and copper reservoirs in the upper $3 \mathrm{ft}$ of the unsaturated zone could eventually migrate to the saturated zone (groundwater).

\section{Temporal Variability}

Water quality in the study area varied over time. Temporal variability in water quality generally was larger at some sites than other sites in the study area and larger for some water-quality constituents (like nitrate, iron, manganese, and fecal streptococcus bacteria) than other constituents. Field contamination (samples collected for inorganic analyses before 1996 or collected for bacteria analyses before 1997) or laboratory error likely account for the few, unusually small or large concentrations of major ions and trace elements reported for some samples from the study area (tables 5 and 6). In general, few sites in the study area had increasing water-quality constituents over time, although increasing concentrations of some constituents could be obscured by the relatively large MRLs used by the laboratory.

Concentrations of nitrate in the groundwater samples fluctuated substantially during 1993-1998. Temporal fluctuations in nitrogen can result from fluctuations in nitrogen sources and nitrogen removals (sinks). Nitrogen sources include atmospheric deposition, decaying plant residue, rocks (such as shale), commercial fertilizers, animal feces, human septage (perhaps associated with the homesteads), or biosolids. Nitrogen sinks include plant uptake, microbial reactions, and water transport. Nitrate concentrations were relatively large and fluctuated considerably during 1993-1998 in samples from three wells: D9, D24, and D25 (fig. 12), all located in the north part of the study area. Nitrate concentration in samples from well D9 was about $13 \mathrm{mg}-\mathrm{N} / \mathrm{L}$ in 1993, decreased to about $2 \mathrm{mg}-\mathrm{N} / \mathrm{L}$ in July 1994, increased to a high of 27 $\mathrm{mg}-\mathrm{N} / \mathrm{L}$ in December 1995, decreased again to $8.4 \mathrm{mg}-\mathrm{N} / \mathrm{L}$ in February 1997, then increased again to about $24 \mathrm{mg}-\mathrm{N} / \mathrm{L}$ in 1998. Nitrate concentration in samples from well D10, a 

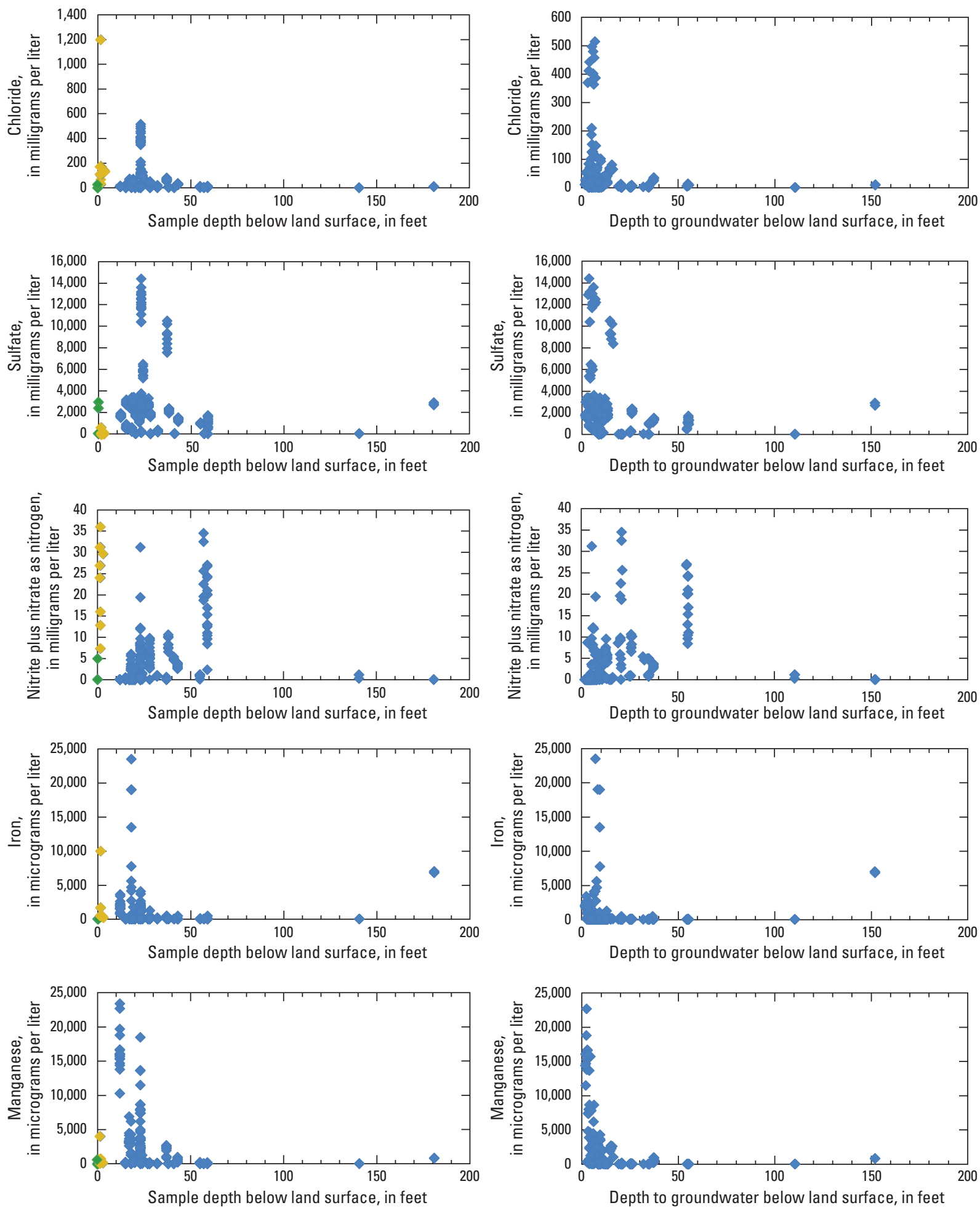

\section{EXPLANATION}

Surface-water sample

Groundwater sample

Figure 11. Water-quality data for selected dissolved constituents compared with sample depth and (for groundwater samples) depth to groundwater near Deer Trail, Colorado, 1993-1998. [Vertical columns of data points in the sample-depth graphs indicate variability in concentration data at the same site (for wells) or site type (if non-well sites)] 

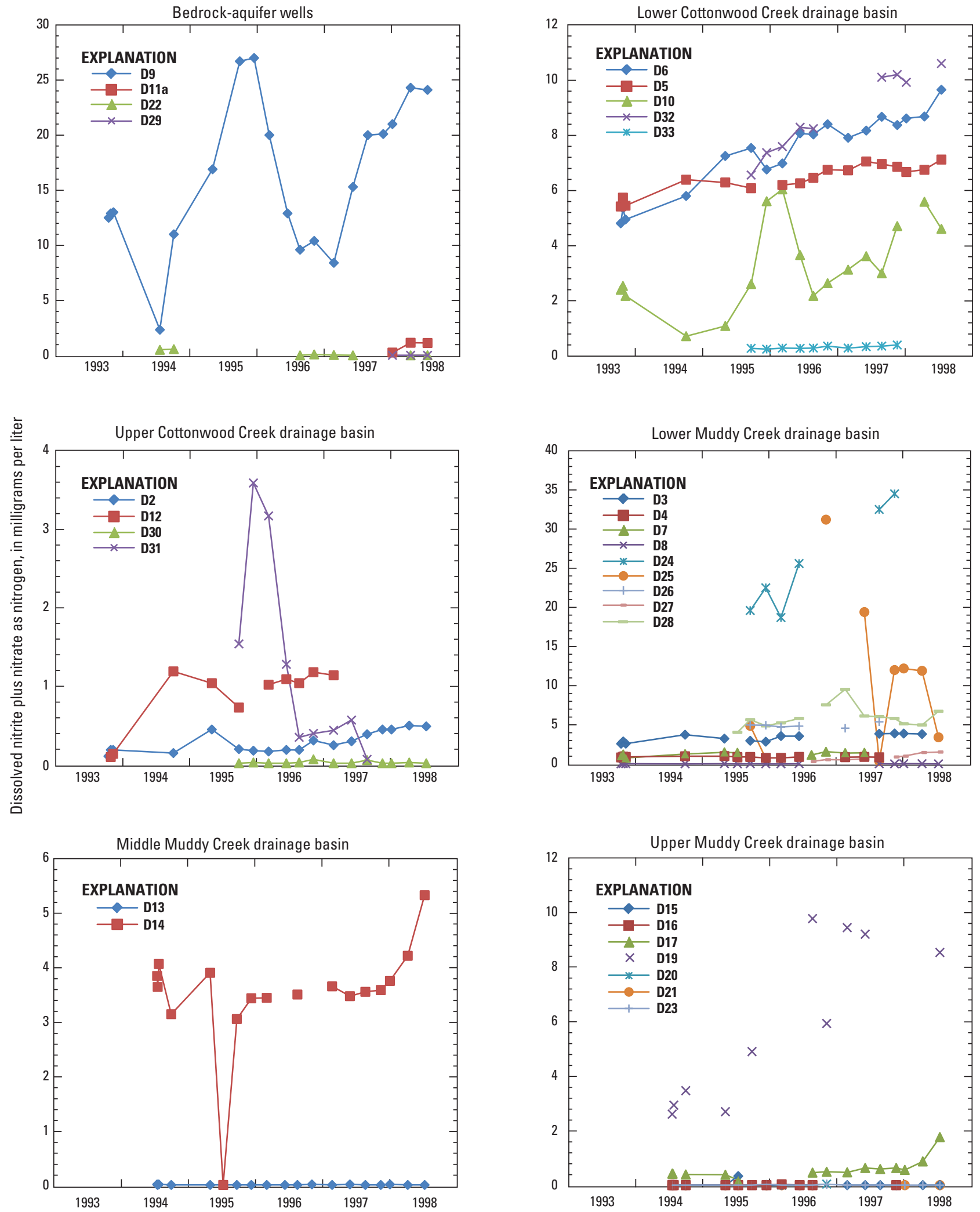

Figure 12. Groundwater concentrations of nitrite plus nitrate as nitrogen over time at monitoring wells near Deer Trail, Colorado, 1993 through 1998. 
shallower well in a different drainage basin, were smaller but had a similar fluctuation pattern (fig. 12). In contrast, nitrate concentration in samples from well D24 generally increased from about $20 \mathrm{mg}-\mathrm{N} / \mathrm{L}$ in September 1995 to $34.5 \mathrm{mg}-\mathrm{N} / \mathrm{L}$ in November 1997. Nitrate concentration in samples from well D25 fluctuated considerably, ranging from about $0.4 \mathrm{mg}-\mathrm{N} / \mathrm{L}$ in August 1997 to about $31 \mathrm{mg}-\mathrm{N} / \mathrm{L}$ in November 1996 (fig. 12; table 5). Patterns in nitrate concentration in groundwater of the study area usually showed that high-concentration periods were followed by low-concentration periods. Nitrate concentrations in samples from wells D6, D24, and D32 increased more markedly and more consistently than in those from other wells in the study area (fig. 12). In general, nitrate concentration increased in samples from most wells along lower Cottonwood Creek (fig. 12) but was more variable in samples from wells D9, D14, D19, D25, D28, and D31.

Concentrations of selected trace elements at some studyarea sites fluctuated over time. Study-area samples were routinely analyzed for dissolved forms of selected trace elements; at most sites, concentrations usually were near or less than laboratory MRLs. Trace elements are difficult to quantify at low concentrations and are present in dust and other airborne constituents, so some trace-element data may be affected by field contamination (if before 1996), laboratory contamination, or analytical interference. The November 1993 concentration of manganese in the sample from well D6, August 1996 concentrations of nickel and zinc in the sample from well D16, the June 1997 concentrations of iron in the samples from wells D7, D27, and D28, and the larger cadmium and chromium concentrations reported for various wells likely resulted from field or laboratory bias. Large concentrations of iron and manganese in samples from wells D15, D16, D21, D23, and D29 likely are representative of the groundwater and are confirmed by later samples. Iron concentration in samples from well D21 ranged from $320 \mu \mathrm{g} / \mathrm{L}$ in 1994 to $23,500 \mu \mathrm{g} / \mathrm{L}$ in 1995 . Manganese concentration in samples from well D21 ranged from $1,250 \mu \mathrm{g} / \mathrm{L}$ in 1996 to $6,200 \mu \mathrm{g} / \mathrm{L}$ in 1994. Groundwater samples from wells D15, D16, and D21 generally had larger dissolved iron concentrations in summer following rain, and groundwater samples from wells D6, D15, and D23 generally had larger dissolved manganese concentrations in early summer before rain (table 5; precipitation data were reported by Yager and Arnold, 2003).

In general, fecal coliform and fecal streptococcus bacteria were not consistently present or absent in groundwater samples, despite multiple sources of these bacteria. Fecal streptococcus bacteria counts ranged from less than 2 colonies per $100 \mathrm{~mL}$ in 1998 to greater than 10,000 colonies per $100 \mathrm{~mL}$ in 1996 in samples from well D2. Fecal streptococcus bacteria counts ranged from 180 colonies per $100 \mathrm{~mL}$ in 1998 to 4,200 colonies per $100 \mathrm{~mL}$ in 1997 in samples from S5, a shallow temporary pond near well D15. The revised sampling methods implemented in 1997 mean that the bacteria data for 1997-1998 likely are not biased by sample-collection methods and, therefore, may be more representative of bacteria concentration than earlier samples. In some cases, these later data seem to confirm earlier bacteria counts at some sites such as in samples from wells D2, D13, and D22. In general, the temporal pattern in the bacteria data is isolated large counts followed by smaller counts or non-detects (table 6). It is not known if laboratory conditions or procedures contributed to the temporal variability present in the bacteria data, although differences in bacteria counts are evident for some replicate (split) samples (the environmental sample and the replicate) (table 6).

Of the limited analytes considered for this study, nitrate increased in concentration the most consistently over time; however, the water-quality data from this study were not suitable for statistical evaluation of temporal trend. Much of the data are derived from analyses that were optimized for higher concentration data, so the data for this study often are censored below relatively large MRLs. In addition, the sampling methods changed substantially over time. Initial sampling methods for baseline and reconnaissance sampling used a less-strict sampling protocol than was used for later samples. This change in sampling preparation and sample-collection methods in late 1995 means that the later water-quality data better represented water quality of the study area, but that water-quality data collected after December 1995 were not strictly comparable to previously collected data. Finally, the power of a trends test is low when sampling frequency was less than about 5 years of quarterly sampling, as in this case (D.R. Helsel, U.S. Geological Survey, oral commun., 2000).

\section{Comparison with Regulatory Standards}

A comparison of water-quality data to regulatory standards provides a context for the concentration values. Values that are larger than the appropriate regulatory standard indicate the water from that site may not be appropriate for a particular use such as drinking water or livestock watering. Regulatory water-quality standards for surface water are not included in this report because few surface-water samples could be obtained. Regulatory water-quality standards for groundwater are included with a summary of the groundwater-quality data in table 8 and figure 13; however, no groundwater-quality standard actually may be enforceable at the routinely sampled sites in the study area. The human health standard may not be enforceable because that standard is based on consumption of the water, and none of the groundwater from the routinely monitored wells was used for drinking-water supply during 1993-1999. In this study area, shallow groundwater generally is more susceptible to contamination from the land surface than deep groundwater. The agricultural standard for groundwater may not be enforceable because the water from the routinely monitored wells was not used for irrigation or livestock watering during 1993-1999. Sites that were sampled occasionally and that actively were used for livestock watering were G1 and G2. Water-quality standards included in this report are from Colorado Department of Public Health and Environment (1997, p. 9-10). These standards and the subsequent discussion comparing study-area groundwater concentrations to the Colorado standards are provided as a frame of reference for 
Table 8. Summary of groundwater-quality data collected for this study near Deer Trail, Colorado, during 1993-1999, and groundwater regulatory limits.

[Data are from analyses of environmental samples by the Metro Wastewater Reclamation District laboratory or from analyses of replicate or environmental samples by the U.S. Geological Survey National Water Quality Laboratory. Standards are from Colorado Department of Health and the Environment (1997); FAO, Food and Agricultural Organization of the United Nations (Pais and Jones, 1997, p. 31); mg/L, milligrams per liter; $\mu \mathrm{g} / \mathrm{L}$, micrograms per liter; --, not applicable; NWQL, U.S. Geological Survey National Water Quality Laboratory; Metro District, Metro Wastewater Reclamation District laboratory]

\begin{tabular}{|c|c|c|c|c|c|c|c|c|c|c|c|}
\hline \multirow[b]{2}{*}{$\begin{array}{l}\text { Chemical } \\
\text { constituent } \\
\text { or property }\end{array}$} & \multicolumn{5}{|c|}{ Summary of data for all samples, all wells } & \multirow[b]{2}{*}{$\begin{array}{l}\text { Analyzing } \\
\text { laboratory }\end{array}$} & \multicolumn{3}{|c|}{ Colorado regulatory standards } & \multirow{2}{*}{$\begin{array}{c}\text { FAO } \\
\text { recommended } \\
\text { maximum } \\
\text { for irrigation } \\
\text { water }\end{array}$} & \multirow[b]{2}{*}{$\begin{array}{l}\text { Well where standard } \\
\text { or guideline was exceeded }\end{array}$} \\
\hline & $\begin{array}{c}\text { Number } \\
\text { of samples } \\
\text { analyzed }\end{array}$ & Units & $\begin{array}{l}\text { Minimum } \\
\text { for all } \\
\text { samples }\end{array}$ & $\begin{array}{l}\text { Maximum } \\
\text { for all } \\
\text { samples }\end{array}$ & $\begin{array}{l}\text { Median } \\
\text { for all } \\
\text { samples }\end{array}$ & & $\begin{array}{l}\text { Human- } \\
\text { health } \\
\text { standards }\end{array}$ & $\begin{array}{c}\text { Secondary } \\
\text { drinking- } \\
\text { water } \\
\text { standards }\end{array}$ & $\begin{array}{l}\text { Agricultural } \\
\text { standards }\end{array}$ & & \\
\hline $\mathrm{pH}$ & 384 & $\begin{array}{l}\text { standard } \\
\text { units }\end{array}$ & 6.2 & 8.6 & 7.2 & field & -- & 6.5 to 8.5 & 6.5 to 8.5 & -- & ${ }^{1} \mathrm{D} 4, \mathrm{D} 15, \mathrm{D} 16, \mathrm{D} 23$ \\
\hline Chloride & 429 & $\mathrm{mg} / \mathrm{L}$ & $<1$ & 515 & 17 & $\begin{array}{l}\text { Metro } \\
\text { District }\end{array}$ & -- & 250 & -- & -- & D6 \\
\hline Fluoride & 14 & $\mathrm{mg} / \mathrm{L}$ & 0.5 & 2 & 1.08 & NWQL & 4.0 & -- & 2.0 & 1.0 & No data exceeded a standard. \\
\hline Sulfate & 429 & $\mathrm{mg} / \mathrm{L}$ & 10 & 14,400 & 1,880 & $\begin{array}{l}\text { Metro } \\
\text { District }\end{array}$ & -- & 250 & -- & -- & $\begin{array}{l}\text { D2, D3, D4, D5, D6, D7, D8 D9, D10, D12, D13, } \\
\text { D14, D15, D16, D20, D21, D22, D23, D25, D27, } \\
\text { D28, D29, D30, D31, D32, D33, G1, G3 }\end{array}$ \\
\hline $\begin{array}{l}\text { Nitrogen, nitrite } \\
\text { as N }\end{array}$ & 429 & $\mathrm{mg} / \mathrm{L}$ & $<0.02$ & 1.1 & $<0.02$ & $\begin{array}{l}\text { Metro } \\
\text { District }\end{array}$ & 1.0 & -- & 10 & -- & D7 \\
\hline $\begin{array}{l}\text { Nitrogen, nitrite } \\
\text { plus nitrate as } \mathrm{N}\end{array}$ & 429 & $\mathrm{mg} / \mathrm{L}$ & $<0.02$ & 34.5 & 0.87 & $\begin{array}{l}\text { Metro } \\
\text { District }\end{array}$ & 10.0 & -- & 100 & -- & D9, D24, D25, D32 \\
\hline Aluminum & 6 & $\mu \mathrm{g} / \mathrm{L}$ & $<1$ & $<5$ & $<2$ & NWQL & -- & -- & 5,000 & 5,000 & No data exceeded a standard. \\
\hline Antimony & 6 & $\mu \mathrm{g} / \mathrm{L}$ & $<1$ & $<5$ & $<2$ & NWQL & 6 & -- & & -- & No data exceeded a standard. \\
\hline Arsenic & 6 & $\mu \mathrm{g} / \mathrm{L}$ & $<1$ & 4 & $<1$ & NWQL & 50 & -- & 100 & 100 & No data exceeded a standard. \\
\hline Barium & 14 & $\mu \mathrm{g} / \mathrm{L}$ & 9 & 57 & 14.71 & NWQL & 2,000 & -- & -- & -- & No data exceeded a standard. \\
\hline Beryllium & 14 & $\mu \mathrm{g} / \mathrm{L}$ & $<0.5$ & $<3$ & $<2$ & NWQL & 4 & -- & 100 & 100 & No data exceeded a standard. \\
\hline Boron & 6 & $\mu \mathrm{g} / \mathrm{L}$ & 101 & 1,590 & 390 & NWQL & & & 750 & & D22 \\
\hline Cadmium & 443 & $\mu \mathrm{g} / \mathrm{L}$ & $<0.02$ & 6 & $<0.2$ & both & 5 & -- & 10 & 10 & D3, D15 (replicates) \\
\hline Chromium & 429 & $\mu \mathrm{g} / \mathrm{L}$ & $<20$ & 430 & $<20$ & $\begin{array}{l}\text { Metro } \\
\text { District }\end{array}$ & 100 & -- & 100 & 100 & $\begin{array}{l}\text { D2, D5, D6, D9, D10, D15, D16, D20, D22, D23, } \\
\text { D28, D29, D30, D31, D32, D33 }\end{array}$ \\
\hline Cobalt & 14 & $\mu \mathrm{g} / \mathrm{L}$ & $<2$ & $<36$ & $<12$ & NWQL & -- & -- & 50 & 50 & No data exceeded a standard. \\
\hline Copper & 429 & $\mu \mathrm{g} / \mathrm{L}$ & $<20$ & $<200$ & $<20$ & $\begin{array}{l}\text { Metro } \\
\text { District }\end{array}$ & -- & 1,000 & 200 & 200 & No data exceeded a standard. \\
\hline Iron & 429 & $\mu \mathrm{g} / \mathrm{L}$ & $<50$ & 23,500 & $<50$ & $\begin{array}{l}\text { Metro } \\
\text { District }\end{array}$ & -- & 300 & 5,000 & 5,000 & D7, D15, D16, D21, D23, D27, D28, D29, D30, G3 \\
\hline Lead & 429 & $\mu \mathrm{g} / \mathrm{L}$ & $<10$ & $<10$ & $<10$ & $\begin{array}{l}\text { Metro } \\
\text { District }\end{array}$ & 50 & -- & 100 & 5,000 & No data exceeded a standard. \\
\hline Lithium & 14 & $\mu \mathrm{g} / \mathrm{L}$ & 29 & 220 & 110 & NWQL & -- & -- & 2,500 & 2,500 & No data exceeded a standard. \\
\hline Manganese & 429 & $\mu \mathrm{g} / \mathrm{L}$ & $<20$ & 23,410 & 220 & $\begin{array}{l}\text { Metro } \\
\text { District }\end{array}$ & -- & 50 & 200 & 200 & $\begin{array}{l}\mathrm{D} 3, \mathrm{D} 4, \mathrm{D} 6, \mathrm{D} 7, \mathrm{D} 8, \mathrm{D} 9, \mathrm{D} 12, \mathrm{D} 13, \mathrm{D} 14, \mathrm{D} 15, \\
\text { D16, D17, D20, D21, D22, D23, D25, D27, D28, } \\
\text { D29, D30, D31, D32, G1, G3 }\end{array}$ \\
\hline Mercury & 6 & $\mu \mathrm{g} / \mathrm{L}$ & $<0.1$ & $<0.1$ & $<0.1$ & NWQL & 2 & -- & 10 & -- & No data exceeded a standard. \\
\hline Nickel & 429 & $\mu \mathrm{g} / \mathrm{L}$ & $<20$ & 2,520 & $<20$ & $\begin{array}{l}\text { Metro } \\
\text { District }\end{array}$ & 100 & -- & 200 & 200 & D4, D6, D12, D16, D32 \\
\hline Selenium & 6 & $\mu \mathrm{g} / \mathrm{L}$ & $<1$ & 4 & $<1$ & NWQL & 50 & -- & 20 & 20 & No data exceeded a standard. \\
\hline Silver & 14 & $\mu \mathrm{g} / \mathrm{L}$ & $<3$ & $<12$ & $<4$ & NWQL & 50 & -- & -- & -- & No data exceeded a standard. \\
\hline Vanadium & 14 & $\mu \mathrm{g} / \mathrm{L}$ & $<6$ & $<30$ & $<24$ & NWQL & -- & -- & 100 & -- & No data exceeded a standard. \\
\hline Zinc & 429 & $\mu \mathrm{g} / \mathrm{L}$ & $<20$ & 5,460 & $<20$ & $\begin{array}{l}\text { Metro } \\
\text { District }\end{array}$ & -- & 5,000 & 2,000 & 2,000 & D16 \\
\hline
\end{tabular}



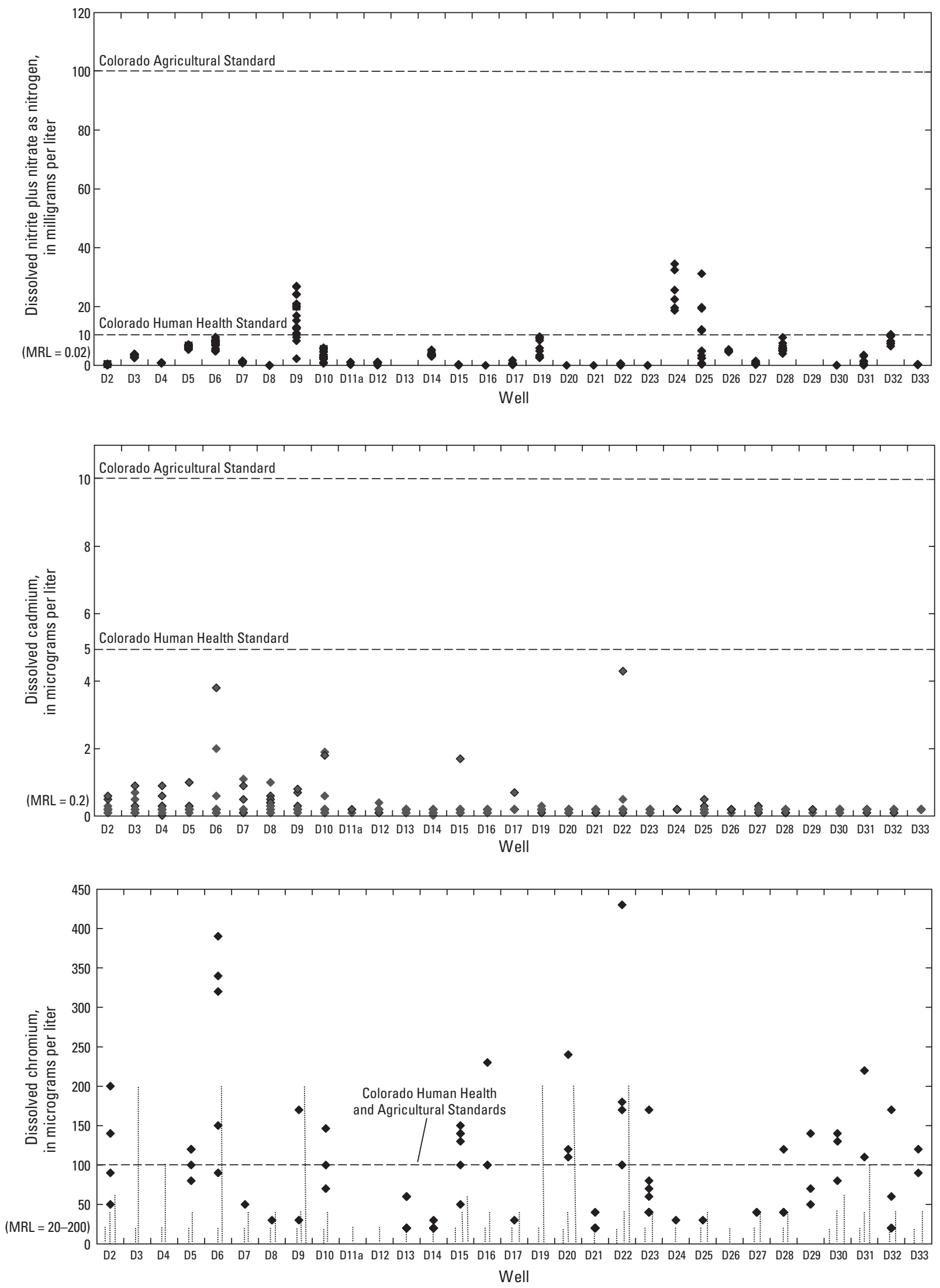

Figure 13. Distribution of groundwater-concentration data for samples collected near Deer Trail, Colorado, compared to regulatory standards for selected constituents, 1993 through 1999. [Where the minimum reporting level (MRL) is distinguishable above the axis, dotted vertical lines represent data that were reported by the laboratory as less than the MRL] 

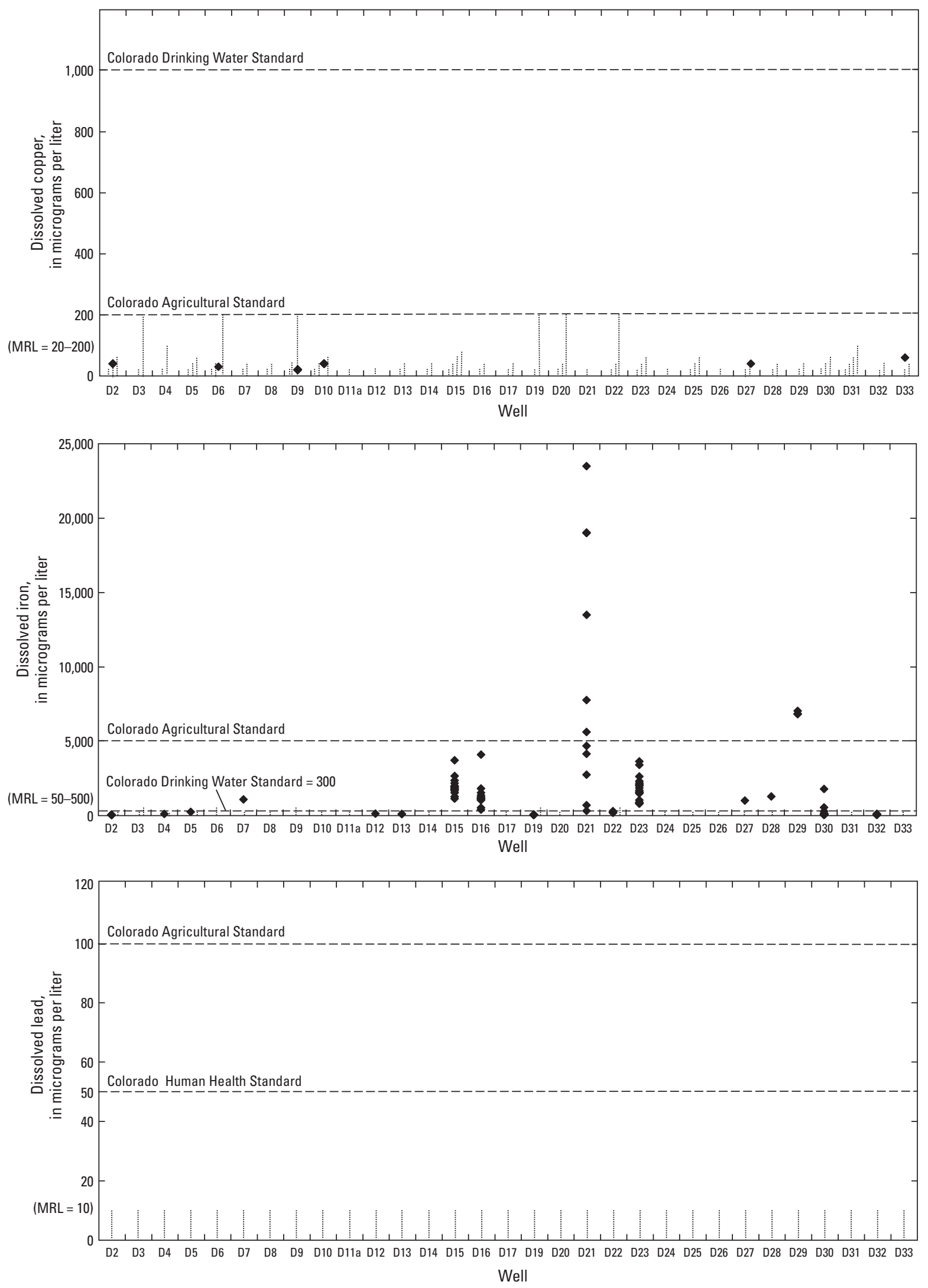

Figure 13. Distribution of groundwater-concentration data for samples collected near Deer Trail, Colorado, compared to regulatory standards for selected constituents, 1993 through 1999. [Where the minimum reporting level (MRL) is distinguishable above the axis, dotted vertical lines represent data that were reported by the laboratory as less than the MRL] —Continued 

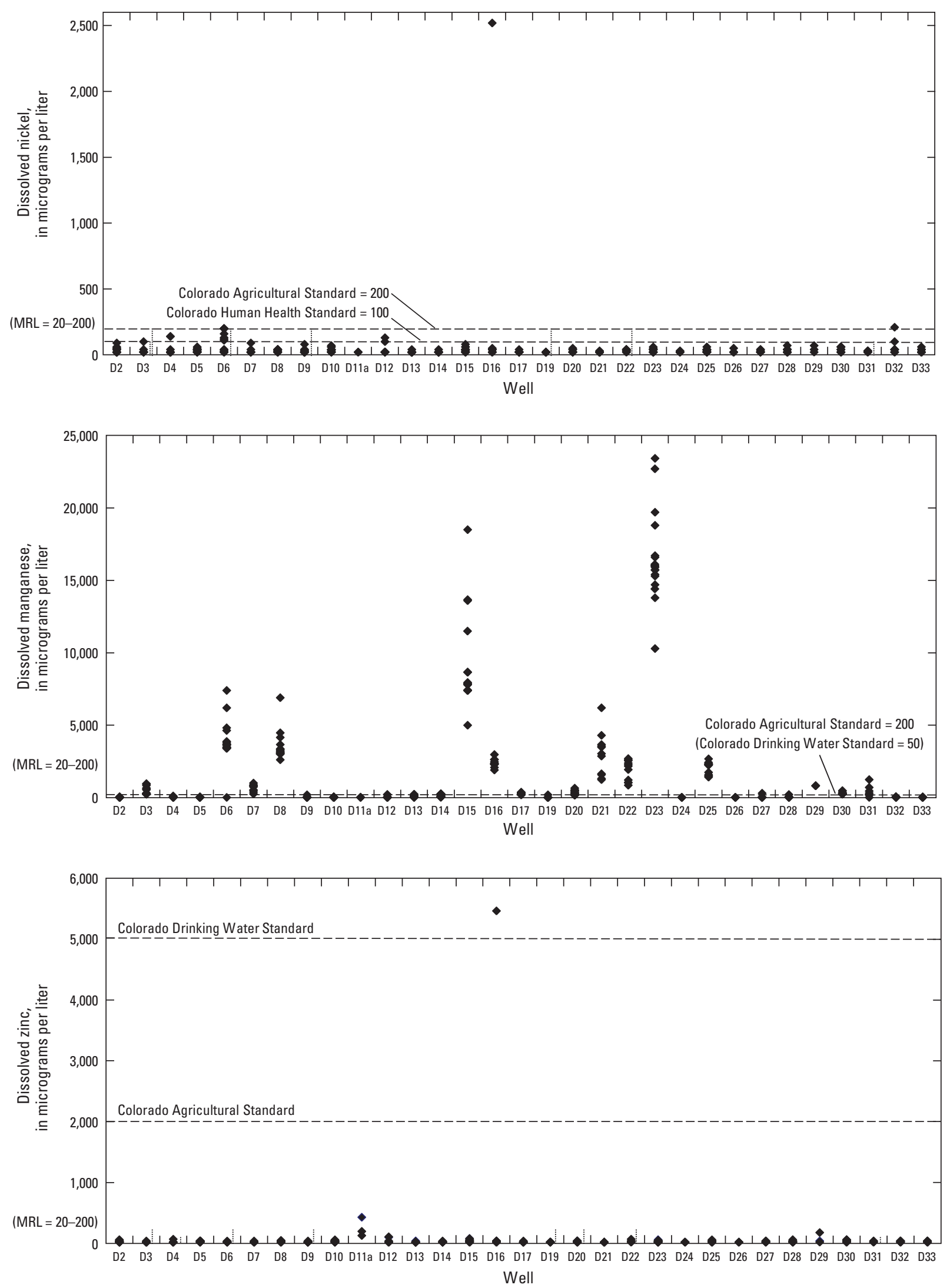

Figure 13. Distribution of groundwater-concentration data for samples collected near Deer Trail, Colorado, compared to regulatory standards for selected constituents, 1993 through 1999. [Where the minimum reporting level (MRL) is distinguishable above the axis, dotted vertical lines represent data that were reported by the laboratory as less than the MRL]—Continued 
the concentration data but do not indicate biosolids effects on water quality. Therefore, all the water-quality data are included in this comparison of regulatory standards. The groundwater samples were not routinely analyzed for all regulated constituents, but at least some of the samples were analyzed for all regulated characteristics and inorganic constituents. During 1993-1999, groundwater samples from the study area exceeded various Colorado regulatory standards.

Colorado has stipulated regulatory limits for some major ions in groundwater (Colorado Department of Public Health and Environment, 1997). Groundwater samples from the study area met the drinking-water standard for chloride of $250 \mathrm{mg} / \mathrm{L}$, except for most samples from well D6 (table 8). The maximum chloride concentration reported for the study-area samples during 1993-1999 was $515 \mathrm{mg} / \mathrm{L}$ at well D6 in March 1996. All groundwater samples from the study area that were analyzed for fluoride met the human-health and agricultural standards (table 8). However, groundwater samples from most routinely monitored wells in the study area including well D6 exceeded the drinking-water standard for sulfate of $250 \mathrm{mg} / \mathrm{L}$ (table 8). The maximum sulfate concentration reported for 1993-1999 was $14,400 \mathrm{mg} / \mathrm{L}$ at well D6 in August 1997. In addition, concentrations of dissolved solids were greater than $10,000 \mathrm{mg} / \mathrm{L}$ in all samples from wells D6 and D22 (table 5), which can limit use of water at these sites.

Most concentrations of nitrate were less than the $10 \mathrm{mg} / \mathrm{L}$ Colorado human-health standard (fig. 13). Concentrations of nitrate exceeded the standard in samples from wells D9, D24, D25, and D32 (fig. 13). Nitrate concentration exceeded this standard for all samples from well D24 and most samples from well D9 (fig. 13). Concentrations of nitrate in baseline samples from well D9 also exceeded the Colorado human health standard. All groundwater data for nitrate from study-area wells were less than the agricultural standard of $100 \mathrm{mg} / \mathrm{L}$ (fig. 13). The maximum nitrate concentration reported for $1993-1999$ was $34.50 \mathrm{mg} / \mathrm{L}$ as $\mathrm{N}$ at well D24 in November 1997 (tables 5 and 8).

Samples were not routinely analyzed for all the regulated trace elements, and sometimes the laboratory reported data as censored at MRLs greater than the standard, in which cases it is not known if the concentration in the sample exceeded the standard. Of the trace elements that were routinely analyzed, concentrations in the groundwater usually were less than Colorado drinking-water standards (table 8). Exceptions include nickel in samples from wells D4, D6, D12, D16, and D32 and chromium, iron, and manganese concentrations in samples from many wells (fig. 13). Concentrations of trace elements in the groundwater were less than Colorado agricultural standards (table 8), except for iron concentrations in samples from wells D21 and D29; nickel concentrations in samples from wells D6, D16, and D32; and manganese concentrations in samples from wells D3, D6, D7, D8, D12, D13, D14, D15, D16, D17, D20, D21, D22, D23, D25, D27, D28, D29, D30, D31, and G3. Concentrations of aluminum, antimony, arsenic, barium, beryllium, cobalt, copper, lead, lithium, mercury, selenium, silver, and vanadium in environmental samples collected in 1999 (table 8) and in replicate samples collected during 1994-1999 from study-area wells all were less than Colorado standards.

Concentrations in baseline groundwater samples exceeded some Colorado regulatory standards; note that these samples were collected before biosolids applications to the study area began. Concentrations of chloride in baseline samples from well D6 and concentrations of sulfate in all baseline samples (except those from well G2) exceeded Colorado drinking-water standards. Concentrations of nitrate in baseline samples from well D9 exceeded the Colorado drinking-water standard. Concentrations of iron and manganese in baseline samples from various monitoring wells in the study area also exceeded Colorado regulatory standards, sometimes by a large margin.

\section{Effects on Water Quality}

Natural factors such as geology, meteorology, and microbial activity can affect water quality. Anthropogenic factors such as biosolids applications also can affect water quality. Many other factors could affect water quality but were not considered in this limited study. Statistical comparisons generally are not applicable for this limited data set. In particular, many more water-quality samples would have had to be collected in order to apply multivariate statistical techniques to the 1993-1999 data set (D.R. Helsel, U.S. Geological Survey, oral commun., 2000). However, specialty sampling and preliminary geochemical modeling can provide information about how various factors are affecting water quality. For this analysis of effects on water quality, all the water-quality data collected for the study during 1993-1999 were included because geological, meteorological, microbiological, and flow-path effects on water quality do not stop when biosolids are applied. These natural processes can still dominate water quality in a biosolids-application area if the biosolids effects are small.

\section{Geological Effects}

Near-surface geological materials in the study area include alluvium, loess, sandstone, siltstone, and shale (fig. 10; Robson and Banta, 1995; Sharps, 1980; Yager and Arnold, 2003). Aquifer-bearing geologic materials in the study area that are within a few hundred feet of the land surface include loess, current or past alluvial deposits, sandstone, and siltstone. Lithologic information for the study area was reported by Yager and Arnold (2003) and Yager and others (2004a, table 7). Some lithologic information for wells D31 and D33 is included in table 9, but detailed lithologic information for the screened interval is not available for most of the monitoring wells. Much more detailed lithologic information for the vicinity of the study area was obtained when the monitoring wells for the USGS Expanded Monitoring Program (fig. 3) were installed. 
Table 9. Chemical data for core samples collected from D31 and D33 boreholes during drilling near Deer Trail, Colorado, May 1995.

[Chemical analyses from Metro Wastewater Reclamation District laboratory; all core was loess obtained from split-spoon auger during drilling of the borehole for the monitoring well; $\mu \mathrm{S} / \mathrm{cm}$, microsiemens per centimeter at 25 degrees Celsius; $\mu \mathrm{g} / \mathrm{g}$, micrograms per gram; (A), available metals from ammonium bicarbonate extraction; (T), total metals; (E), extractable by using sodium bicarbonate; <, less than]

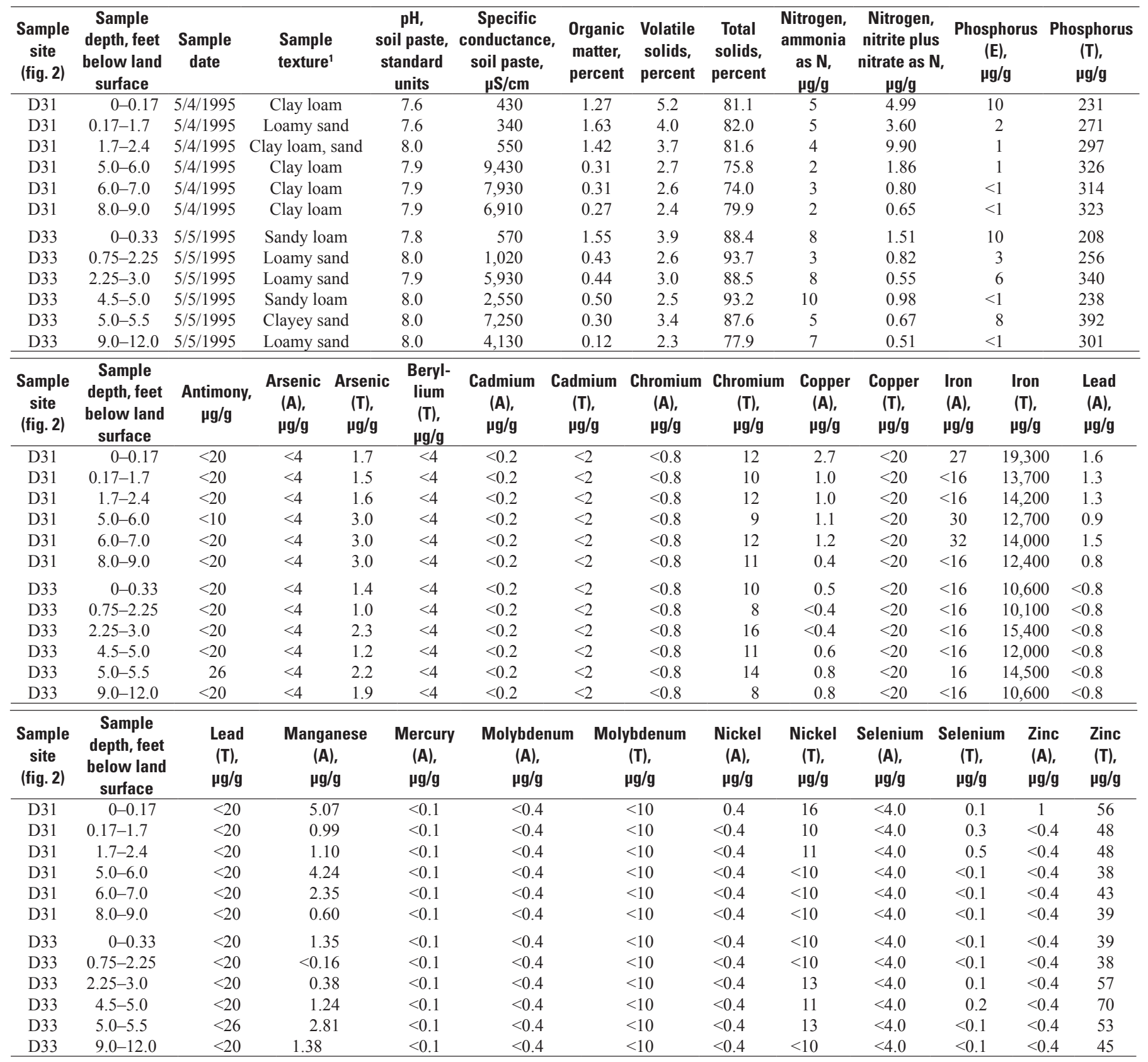

${ }^{1}$ From geologist's description of core. 
Geology affected water quality in the study area. Majorion and trace-element chemistry of water in the study area appears to be controlled primarily by local geology. From core obtained during well installations in 1999-2002, it was observed that geologic units mapped as sandstone in figure 8 are interbedded with thin layers of shale in the vicinity of the study area, contain iron-rich concretions, and weather chemically to produce secondary crystalline minerals and amorphous iron oxides. Chemical analysis of core samples (table 9) confirmed the presence of many of the same major and trace elements in the near-surface geologic materials of the study area that were in the groundwater. Iron concentrations in the loess samples were large (as much as 19,300 $\mu \mathrm{g} / \mathrm{g}$ ) (table 9). In some places, loess was observed to overly the alluvium from buried paleo channels; in other places, the loess occurs as terraces or as flood plains with incised alluvial systems. The alluvium was igneous (granitic sands and gravels derived from Rocky Mountain glacial outwash) or sedimentary (reworked pebbles and sands from local sandstones, siltstones, and shales). Contact with groundwater and infiltrating surface water results in dissolution, precipitation, and weathering reactions of the minerals in these rocks that change the chemical composition of the groundwater (Drever, 1988). Also, iron and manganese oxides that form naturally in the dry, oxygenated soils of the study area can sorb and later release various elements that leach from surface sources like biosolids (T.P. Wilson, U.S. Geological Survey, written commun., May 29, 2012). Localized variations in lithology, weathering, mineralogy, and residence time in the aquifers can cause the variations in groundwater major-ion and trace-element concentrations observed in the 1993-1999 data from this study area. These localized variations also explain why major-ion groundwater concentrations were not uniform within the geologic-formation boundaries shown in figure 10, but likely account for the major-ion and trace-element composition of the water in the study area.

Calcium and sulfate were major ions in the water collected from the study area (fig. 9), and the source of these ions likely was the near-surface geologic materials in the study area. Calcium content in loess samples was about 1-2 percent but as high as about 10.5 percent in loess that had gypsum crystals in contact with groundwater near well D6 (Yager and others, 2004a, table 7). Sulfur content was less than 0.05-1.5 percent in loess samples from the study area; smaller sulfur content was observed in loess samples that contained visible coal fragments (Yager and others, 2004a, table 7). Sulfur content was slightly larger (1.8 percent) in weathered shale in the vicinity of well D9. In general, sulfur content in shale is about twice that in sandstone, but calcium content is similar in sandstone and shale (Hem, 1992, p. 5). Sulfur in water of the study area during 1993-1999 was primarily in the form of sulfate (tables 5 and 10). Precipitation of calcium sulfate or calcium carbonate minerals will decrease the concentrations of calcium and sulfate that are dissolved in the water, which can account for some of the spatial variability of calcium and sulfate in groundwater of the study area. The dissolution of gypsum $\left(\mathrm{CaSO}_{4} \cdot 2 \mathrm{H}_{2} \mathrm{O}\right)$ could account for much of the calcium and sulfate ions, and dissolution of calcite $\left(\mathrm{CaCO}_{3}\right)$ could account for calcium and bicarbonate ions in the groundwater. Calcium concentrations ranged from about $50 \mathrm{mg} / \mathrm{L}$ in samples from wells D11a and D17 to about $600 \mathrm{mg} / \mathrm{L}$ in samples from well D29, and calcium concentrations fluctuated the most at well D9 (table 5). Sulfate concentrations ranged from less than $100 \mathrm{mg} / \mathrm{L}$ in samples from wells D11a, D17, and D19 to as much as $14,400 \mathrm{mg} / \mathrm{L}$ in samples from well D6, but fluctuated little over time in samples from most wells (table 5). Sulfate also is important in redox reactions as an energy source to groundwater microorganisms after oxygen and nitrate are depleted (see Microbiological Effects section later in this report).

Chloride and sodium were major ions in the water of the study area (fig. 9); at least one source of these ions likely was the near-surface geologic materials in the study area. Chloride content of geologic materials from the study area was not measured, but sedimentary rocks (such as those near the surface in the study area) can be a source of chloride in water (Hem, 1992). Sodium content of geologic materials from the study area was measured at $0.45-0.75$ percent (Yager and others, 2004a). In general, chloride content is about 10 times larger in shales than sandstones, but sodium content is less than 2 times larger in shales than sandstones (Hem, 1992, p. 5). Chloride can be contributed by the weathering of chloride minerals such as halite $(\mathrm{NaCl})$. Sodium can be contributed by the weathering of various silicate minerals. Chloride and sodium also can be contributed by residual salt in marine sediments such as those of the bedrock aquifer, especially in shale (Hem, 1992, p. 118). In general, concentrations of chloride and sodium were least in samples from wells D9 and D11a and most in samples from well D6.

Iron and manganese are trace elements that occur in large concentrations in water samples from some sites in the study area (figs. 8 and 13). The source of these elements likely was the near-surface geologic materials in the study area. Iron content in loess samples from the study area was $0.7-8.3$ percent; the largest iron content measured in geologic materials from the study area was in samples of granitic alluvium and weathered shale (Yager and others, 2004a). The (total) iron content in loess samples from D31 and D33 ranged from $10,100-19,300 \mu \mathrm{g} / \mathrm{g}$; the largest of these concentrations was measured in the top soil layer at D31 (table 9). Drever (1988) reported iron as a major constituent in both typical sandstones and typical shales, but Hem (1992, p. 5) reported that iron content of shales generally is about 2 times that of sandstones. Dissolution of pyrite $\left(\mathrm{FeS}_{2}\right)$ likely is a source of iron and a source of sulfur that becomes sulfate in water of the study area. Manganese content in loess samples from the study area was $102-2,360 \mathrm{PPM}(\mathrm{mg} / \mathrm{kg}$ or $\mu \mathrm{g} / \mathrm{g})$; the largest manganese content was in loess and granitic alluvium samples collected near well D6 (Yager and others, 2004a). The (available) manganese content in loess from the geologic materials from D31 and D33 ranged from 0.38 to $5.07 \mu \mathrm{g} / \mathrm{g}$ (table 9); the highest of these concentrations again was measured in the top soil layer at D31. Drever (1988) reported the manganese of typical shale to be about 17 times that of typical sandstone and almost twice that of typical granite, but Hem (1992, p. 5) reported 
Table 10. Concentrations of redox-sensitive constituents and terminal electron-acceptor processes for groundwater from selected wells near Deer Trail, Colorado, July 1999.

[Samples collected July 12 through July 14, 1999; other water-quality data associated with these samples are listed in table 7; mm Hg, millimeters of mercury; mg/L, milligrams per liter; $\mathrm{H}_{2}$, hydrogen; nmol/L, nanomoles per liter; $\mathrm{NO}_{3}$, nitrate as nitrogen; dissolved, from filtered samples; $\mathrm{SO}_{4}$, sulfate; $\mu \mathrm{g} / \mathrm{L}$, micrograms per liter; TEAP, terminal electron-accepting process; <, less than; Fe ${ }^{3+}$, ferric iron; $\mathrm{O}_{2}$, oxygen; USGS, U.S. Geological Survey]

\begin{tabular}{|c|c|c|c|c|c|c|c|c|c|c|c|c|}
\hline \multirow[b]{2}{*}{$\begin{array}{l}\text { Sample } \\
\text { site } \\
\text { (fig. 2) }\end{array}$} & \multirow{2}{*}{$\begin{array}{c}\text { Air } \\
\text { temperature, } \\
\text { degrees } \\
\text { Celsius }\end{array}$} & \multirow[b]{2}{*}{$\begin{array}{c}\text { Barometric } \\
\text { pressure, } \\
\mathrm{mm} \mathrm{Hg}\end{array}$} & \multicolumn{4}{|c|}{ Electron acceptors } & \multirow[b]{2}{*}{$\begin{array}{c}\mathrm{H}_{2^{\prime}} \\
\mathrm{nmol} / \mathrm{L}\end{array}$} & \multicolumn{2}{|c|}{ Final products } & \multirow[b]{2}{*}{$\begin{array}{l}\text { Predominant } \\
\text { TEAP }^{2}\end{array}$} & \multirow[b]{2}{*}{$\begin{array}{c}\text { Evidence for } \\
\text { TEAP determination }\end{array}$} & \multirow{2}{*}{$\begin{array}{c}\text { Additional } \\
\mathrm{NO}_{3} \text { likely to } \\
\text { be reduced at } \\
\text { this site }\end{array}$} \\
\hline & & & $\begin{array}{c}\text { Dissolved } \\
\text { oxygen }{ }^{1} \text {, } \\
\text { mg/L }\end{array}$ & $\begin{array}{c}\mathrm{NO}_{3^{\prime}} \\
\text { dissolved, } \\
\mathrm{mg} / \mathrm{L}\end{array}$ & $\begin{array}{c}\text { Iron, } \\
\text { dissolved, } \\
\mu \mathrm{g} / \mathrm{L}\end{array}$ & $\begin{array}{c}\mathrm{SO}_{4^{\prime}} \\
\text { dissolved, } \\
\mathrm{mg} / \mathrm{L}\end{array}$ & & $\begin{array}{c}\text { Hydrogen } \\
\text { sulfide, } \\
\text { mg/L }\end{array}$ & $\begin{array}{c}\text { Methane, } \\
\text { mg/L }\end{array}$ & & & \\
\hline D15 & 36 & 632 & $<0.1$ & $<0.05$ & 2,100 & 2,700 & 0.2 & $<0.01$ & $<0.1$ & $\mathrm{Fe}^{3+}$ Reduction & $\mathrm{H}_{2}$ Concentration & Yes \\
\hline D16 & 38 & 628 & 0.1 & $<0.05$ & 910 & 2,200 & 0.5 & $<0.01$ & $<0.1$ & $\mathrm{Fe}^{3+}$ Reduction & $\mathrm{H}_{2}$ Concentration & Yes \\
\hline $\mathrm{D} 21$ & 38 & 627 & 0.2 & $<0.05$ & 7,200 & 250 & 0.1 & $<0.01$ & $<0.1$ & $\mathrm{Fe}^{3+}$ Reduction & $\mathrm{H}_{2}$ and iron concentrations & Yes \\
\hline D22 & 34 & 634 & 0.4 & $<0.05$ & 200 & 9,000 & 1.1 & $<0.01$ & $<0.1$ & $\mathrm{SO}_{4}$ Reduction & $\mathrm{H}_{2}$ Concentration & Yes \\
\hline D23 & 32 & 627 & 0.4 & $<0.05$ & 2,400 & 1,700 & 0.3 & $<0.01$ & $<0.1$ & $\mathrm{Fe}^{3+}$ Reduction & $\begin{array}{l}\mathrm{H}_{2} \text { Concentration and electron- } \\
\text { acceptor consumption }\end{array}$ & Yes \\
\hline D30 & 32 & 635 & 0.3 & $<0.05$ & 120 & 3,000 & 1.8 & $<0.01$ & $<0.1$ & $\mathrm{SO}_{4}$ Reduction & $\begin{array}{l}\mathrm{H}_{2} \text { Concentration and electron- } \\
\text { acceptor consumption }\end{array}$ & Yes \\
\hline DTX2 $2^{3}$ & 30 & 635 & 0.8 & $<0.05$ & 270 & 2,400 & 0.5 & $<0.01$ & $<0.1$ & $\mathrm{O}_{2}$ and $\mathrm{Fe}^{3+}$ Reduction & $\mathrm{H}_{2}$ Concentration & Possibly \\
\hline DTX10 $0^{4}$ & 36 & 634 & 0.7 & $<0.05$ & 3,700 & 1,900 & Offscale $^{5}$ & 0.011 & $<0.1$ & $\mathrm{O}_{2}$ and $\mathrm{SO}_{4}$ Reduction & $\begin{array}{l}\mathrm{H}_{2} \text { Concentration and final- } \\
\text { product production }\end{array}$ & Possibly \\
\hline
\end{tabular}

${ }^{1}$ Iodometric method using the Alsterberg-Azide modification to the Winkler titration procedure as described by U.S. Geological Survey (variously dated).

${ }^{2}$ From Chapelle and others (1995), Lovely and others (1994), and McMahon and Chapelle (2008).

${ }^{3}$ Alluvial-aquifer monitoring well from subsequent USGS monitoring program. This well is shown in figure 3 and is located about 5 miles north of well D30 in the Badger Creek drainage basin.

${ }^{4}$ Zone A (upper) of bedrock-aquifer monitoring well from subsequent USGS monitoring program. This well is shown in figure 3 and is located about 6 miles north of well D15 in the Muddy Creek drainage basin.

${ }^{5} \mathrm{H}_{2}$ in this sample was above analytical quantification, but the $\mathrm{H}_{2}$ concentration could be affected by residual contamination from the submersible pump used to sample this well the previous day. 
that manganese content of shales generally is less than 2 times larger than that of sandstones. By far, the largest iron concentrations in groundwater at the study area were measured in samples from well D21, whereas the largest manganese concentrations were measured in samples from wells D15 and D23 (table 5; fig. 13).

Other trace elements that are present in study-area water also can be contributed by geologic materials. Trace elements can be contributed to groundwater from a variety of silicate, carbonate, and sulfide minerals. Core samples collected at D31 and D33 contained detectable concentrations of arsenic, chromium, copper, iron, manganese, nickel, selenium, and zinc (table 9). Core samples collected at D31 also contained detectable concentrations of lead (table 9). A core sample collected at D33 also contained a detectable concentration of antimony (table 9). These elements typically are present in sedimentary rocks (Drever, 1988, p. 328-329; Hem, 1992, p. 5-6). Drever (1988) and Hem (1992) reported the antimony, arsenic, chromium, copper, lead, nickel, and zinc content of typical shale to be at least 2.5 times that of typical sandstone. The largest chromium concentrations in groundwater were measured in samples from wells D6 and D22 (table 5). Arsenic, copper, lead, and zinc were not routinely detected above the MRLs in groundwater samples from the study area, but arsenic, copper, and zinc were detected in the groundwater samples collected in 1999 and analyzed by using lower MRLs (table 7).

Milliequivalent ratios can indicate whether dissolution of some simple minerals likely accounts for the concentrations of those constituents detected in the groundwater (D.K. Nordstrom, U.S. Geological Survey, oral commun., March 22, 2000). Concentrations of mineral-forming ions can be graphed in units of milliequivalents per liter (meq/L) to evaluate concentration ratios. Gypsum, calcite, dolomite, and halite likely were present in the rocks of the study area. These minerals mostly are composed of three or less major ionic compounds, so the minerals can be represented simply in graphical form. Gypsum and anhydrite are composed of calcium sulfate, so a 1:1 ratio of calcium to sulfate in the groundwater indicates that gypsum (or anhydrite) dissolution likely accounts for those concentrations. Calcite and aragonite are composed of calcium carbonate, so a 1:1 ratio of calcium to bicarbonate in the groundwater indicates that calcite (or aragonite) dissolution likely accounts for those concentrations. Dolomite is composed of calcium, magnesium, and carbonate, so a 1:1 ratio of calcium plus magnesium to bicarbonate in the groundwater indicates that dolomite dissolution likely accounts for those concentrations. Halite is composed of sodium chloride, so a 1:1 ratio of sodium to chloride in the groundwater indicates that halite dissolution likely accounts for those concentrations. If concentrations of one of these major constituents in is greater than that of the other mineral-forming constituent in the groundwater, than dissolution of other minerals or another source of that constituent is indicated.

The distribution of major constituents for selected wells is shown in figure 14 by mineral. Dissolution of gypsum (or anhydrite) likely accounts for most of the calcium and sulfate concentrations in samples from wells D3, D4, D7, D9, D13, D20, D21, D23, D25, D27, D28, and D32 (fig. 14). Gypsum was present at well D6, and gypsum dissolution may account for much of the calcium in samples from well D6 but accounts for little of the sulfate. Dissolution of calcite and dolomite likely account for most of the calcium, magnesium, and bicarbonate concentrations in samples from wells D11a, D14, D17, D19, D21, D24, and D26. Dissolution of halite could account for some of the sodium and chloride in samples from wells D4, D14, and D19; dissolution of halite may account for all the chloride concentration in the study area, but additional sources of sodium (such as from dissolution of feldspar and clay minerals) are indicated from the ion plots. In samples from wells D7, D9, D23, D25, and D29, sulfate or magnesium have been contributed from an additional source, and calcium-magnesium ion exchange may be affecting water quality. The ion plots in figure 14 also indicate that dissolution of a magnesium-sulfate mineral such as epsomite or kieserite could account for most of the magnesium and sulfate concentrations in samples from wells D2, D6, D10, D17, D19, D22, D26, D29, D30, D31, and D33. In samples from wells D6 and D22, dissolution of gypsum and calcite likely were processes contributing calcium, sulfate, and bicarbonate to groundwater, but sulfate was contributed from an additional source such as the oxidation of pyrite (an iron-sulfide mineral) or coal, and magnesium was contributed from an additional source besides dolomite (such as from epsomite, biotite, or clay minerals). In samples from well D22, an additional source of calcium such as feldspars or clay minerals also is indicated. In samples from wells D5, D8, D15, and D16, the geochemistry is more complex and cannot be produced from simple dissolution of just gypsum, calcite, dolomite, halite, or epsomite. In general, the ion plots indicate predominantly gypsum dissolution on the west side of the study area and predominantly epsomite (or another magnesium-sulfate mineral) dissolution on the east side of the study area. Thus, ion plots of water-quality data for 1993-1998 indicate that mineral dissolution could account for much of the major-ion and trace-element chemistry of water (fig. 14).

Major-ion groupings and the chemical response of groundwater to precipitation are other indicators that geology affected water quality. As mentioned in the Spatial Variability section, well groupings indicated by major-ion chemistry (fig. 9) are fairly consistent with the geologic units mapped for the study area by Sharps (1980) (fig. 10). Major-ion concentrations at most wells temporarily decreased after prolonged, intense rainfall (as at wells D2, D6, D9, and D23; Yager and Arnold, 2003), but then concentrations increased again to previous levels as groundwater concentrations re-equilibrated with local geochemistry. This apparent short-term dilution effect on groundwater quality is consistent with geology providing most of the solutes to groundwater. The longer the residence time of a parcel of groundwater in contact with geologic materials, the more the dissolution, precipitation, and weathering reactions in the aquifer are likely to affect groundwater quality (Drever, 1988). 
A
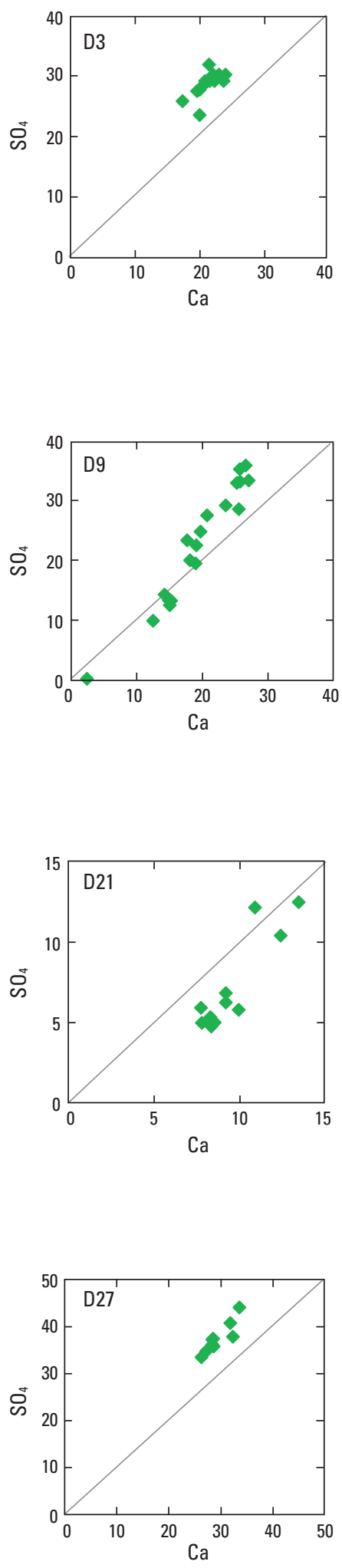

Gypsum $\left(\mathrm{CaSO}_{4} \cdot 2 \mathrm{H}_{2} \mathrm{O}\right)$ or anhydrite $\left(\mathrm{CaSO}_{4}\right)$
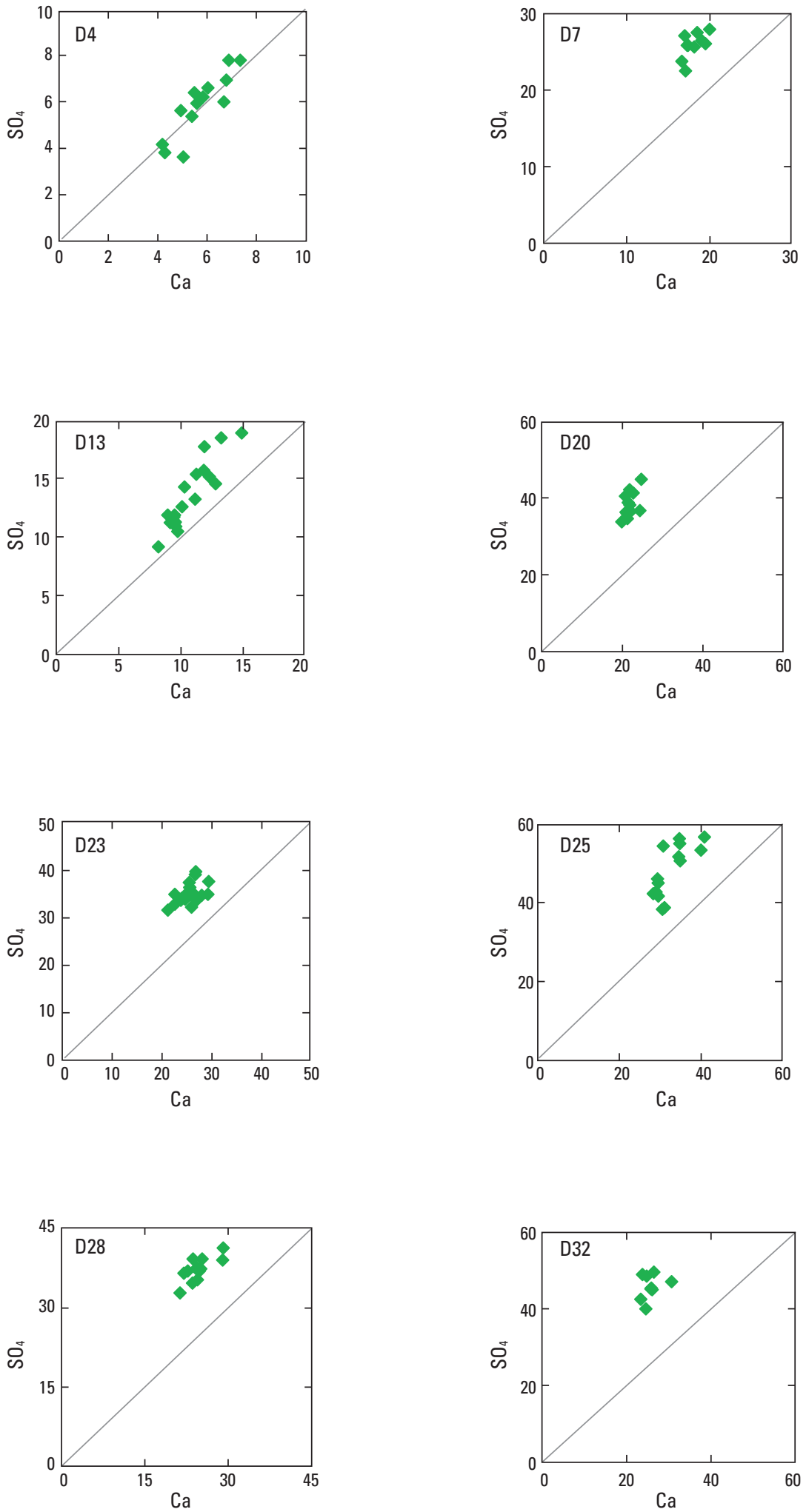

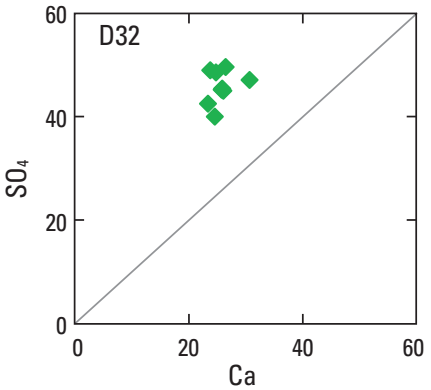

Figure 14. Ion plots showing milliequivalent ratios for $(A)$ gypsum, $(B)$ calcite, $(C)$ dolomite, $(D)$ halite, and $(E)$ epsomite (or another magnesium-sulfate mineral) from samples collected from selected monitoring wells near Deer Trail, Colorado, 1993 through 1998. [All concentrations are shown in milliequivalents per liter; diagonal line represents 1:1 concentration ratio] 
B
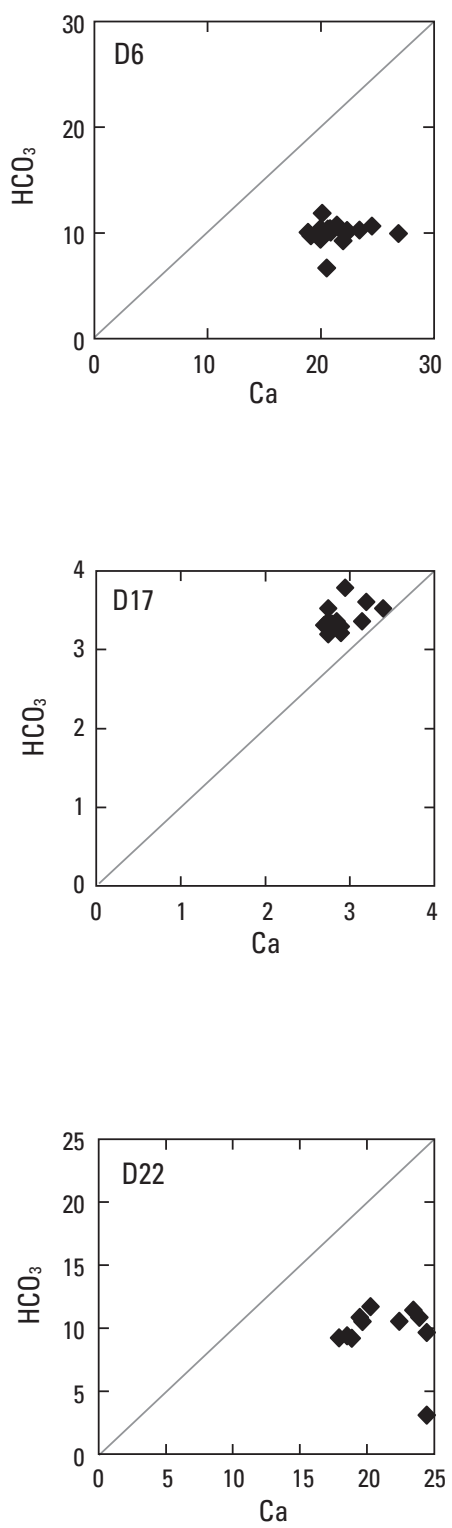

Calcite or aragonite $\left(\mathrm{CaCO}_{3}\right)$
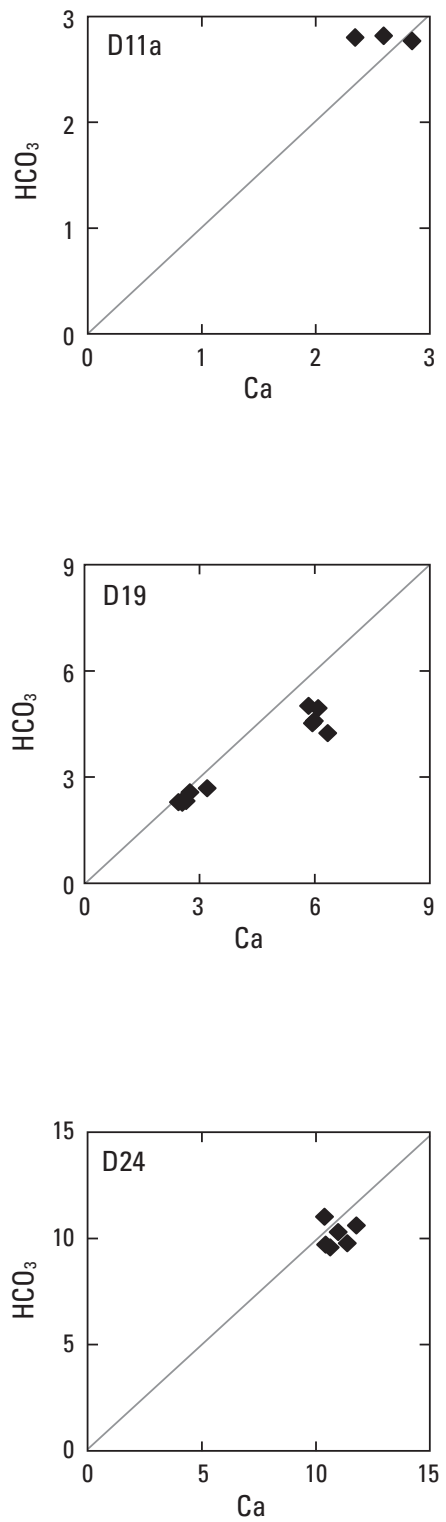
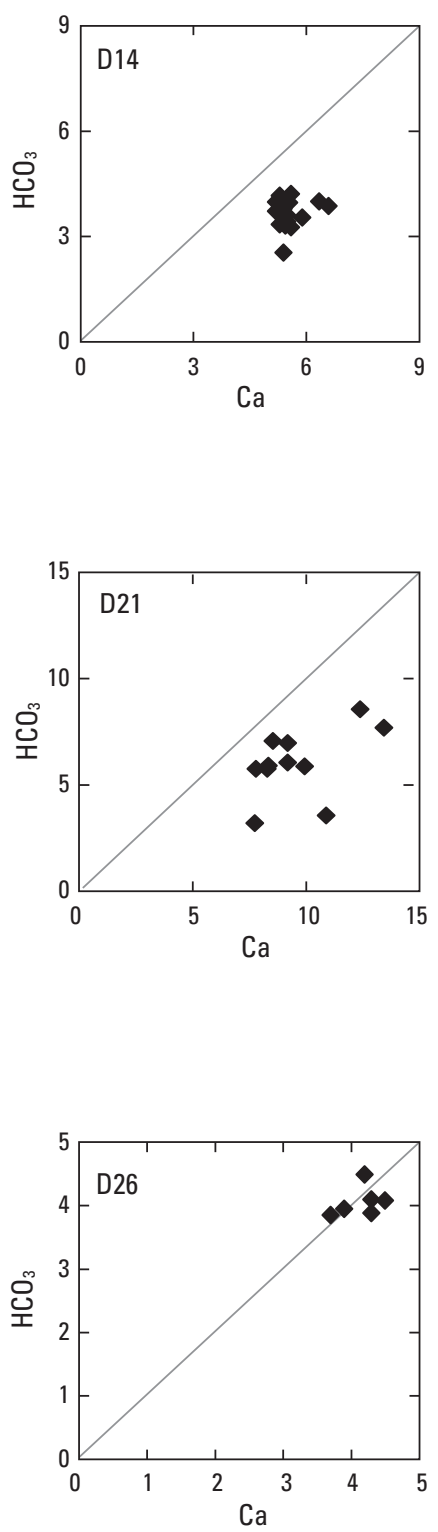

Figure 14. Ion plots showing milliequivalent ratios for $(A)$ gypsum, $(B)$ calcite, $(C)$ dolomite, $(D)$ halite, and $(E)$ epsomite (or another magnesium-sulfate mineral) from samples collected from selected monitoring wells near Deer Trail, Colorado, 1993 through 1998.

[All concentrations are shown in milliequivalents per liter; diagonal line represents 1:1 concentration ratio]—Continued 
$\boldsymbol{C}$
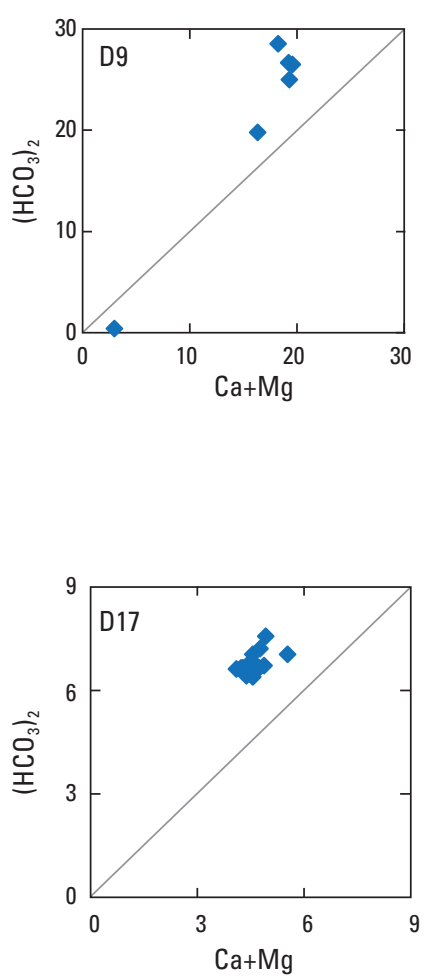

Dolomite $\left(\mathrm{CaMg}\left(\mathrm{CO}_{3}\right)_{2}\right)$
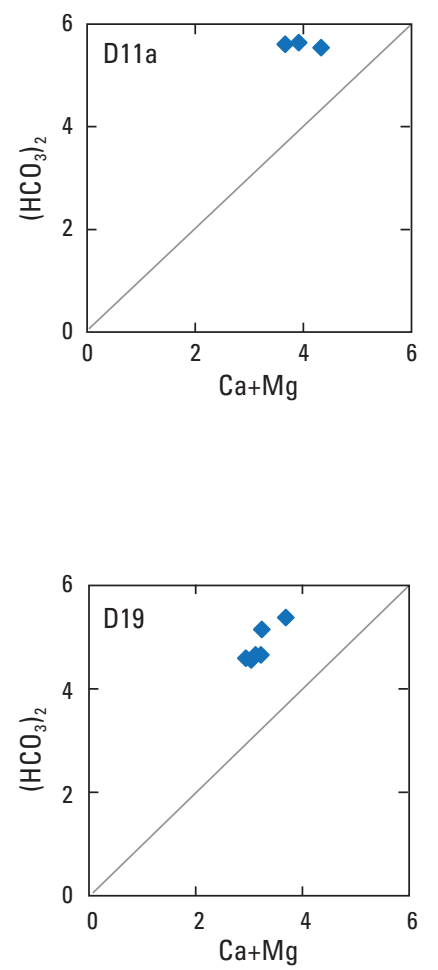
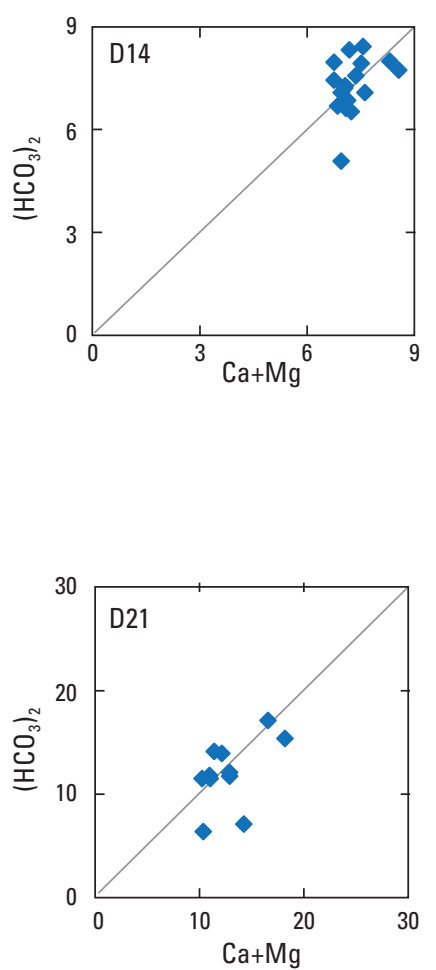
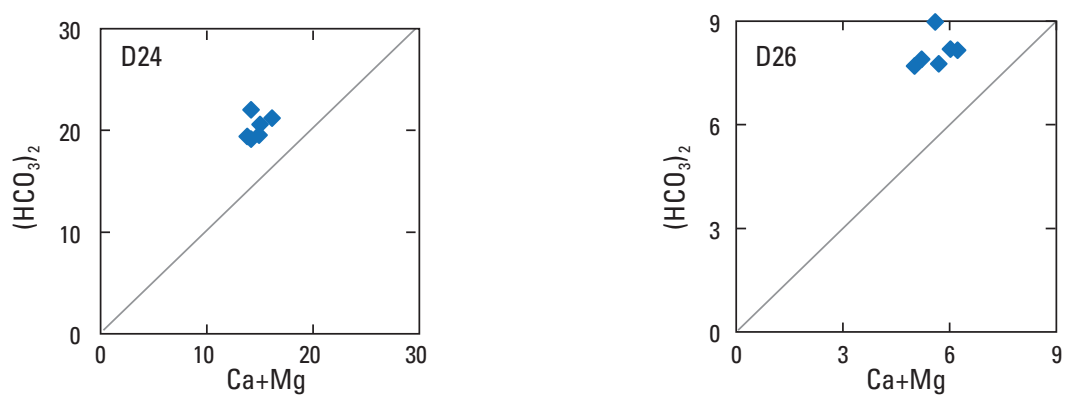

$\boldsymbol{D}$

Halite (NaCl)
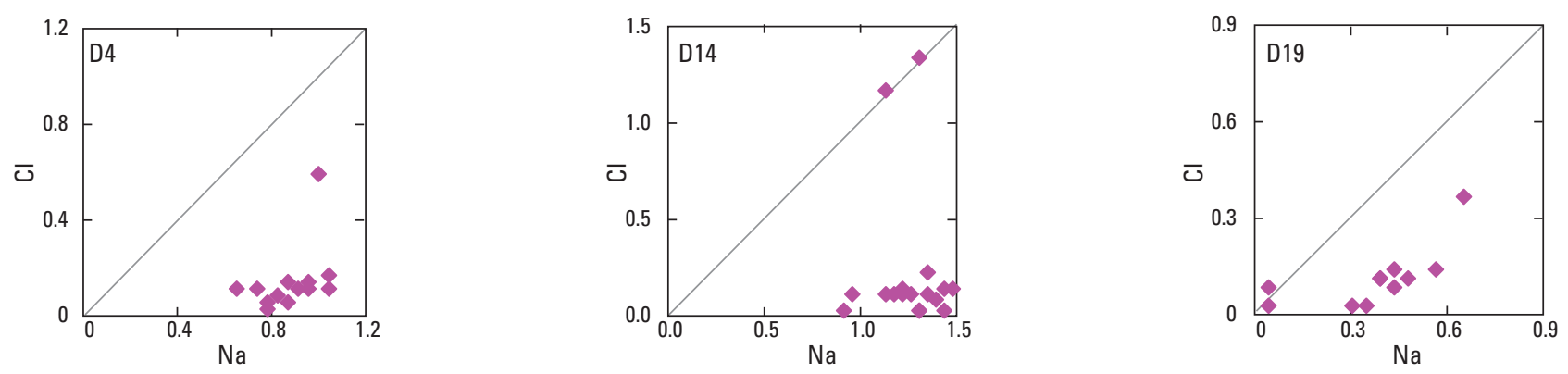

Figure 14. Ion plots showing milliequivalent ratios for $(A)$ gypsum, $(B)$ calcite, $(C)$ dolomite, $(D)$ halite, and $(E)$ epsomite (or another magnesium-sulfate mineral) from samples collected from selected monitoring wells near Deer Trail, Colorado, 1993 through 1998.

[All concentrations are shown in milliequivalents per liter; diagonal line represents 1:1 concentration ratio]—Continued 
$E$
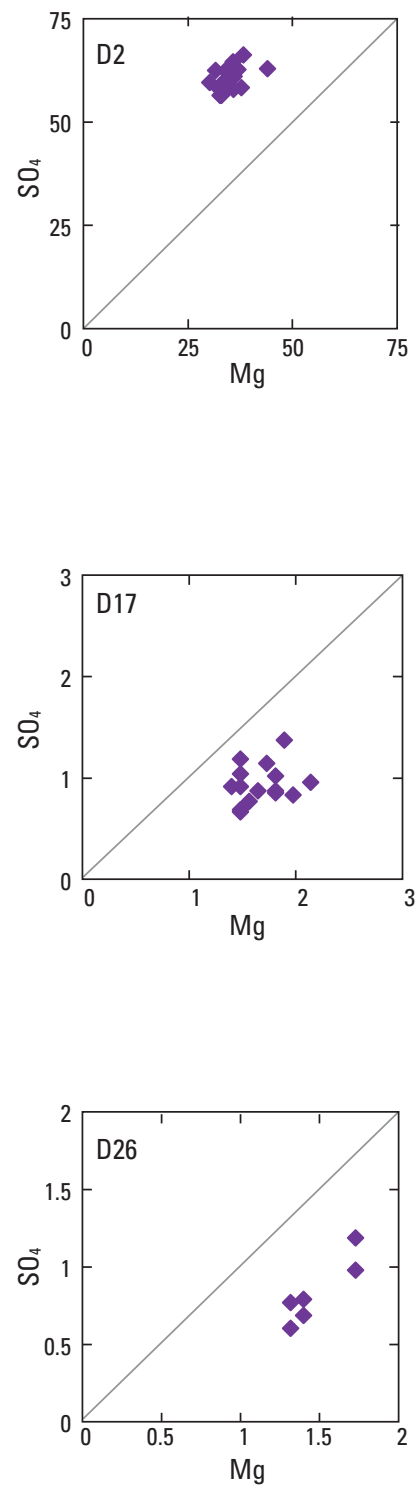

Epsomite $\left(\mathrm{MgSO}_{4} \cdot 7 \mathrm{H}_{2} \mathrm{O}\right)$ or other magnesium-sulfate mineral
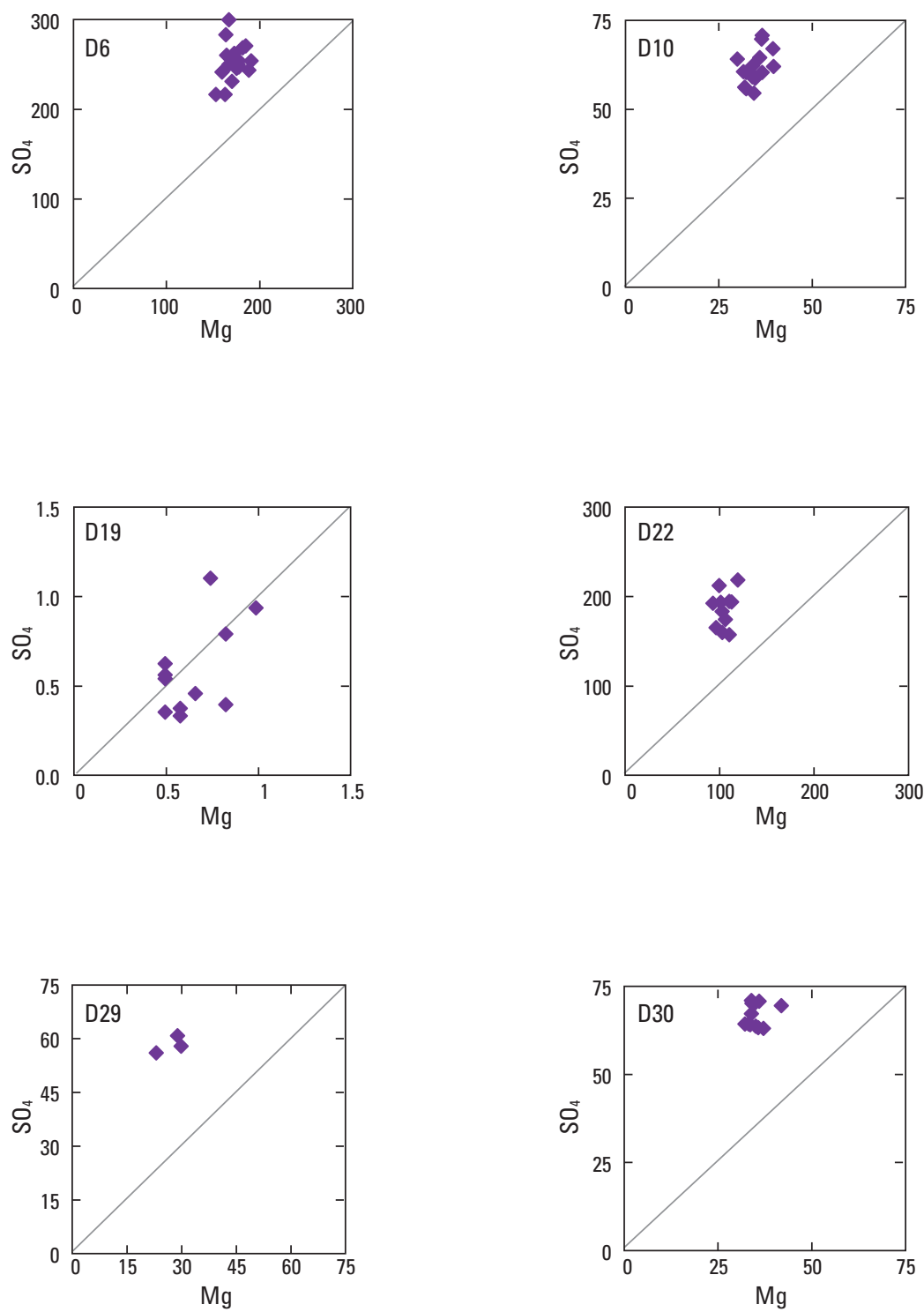
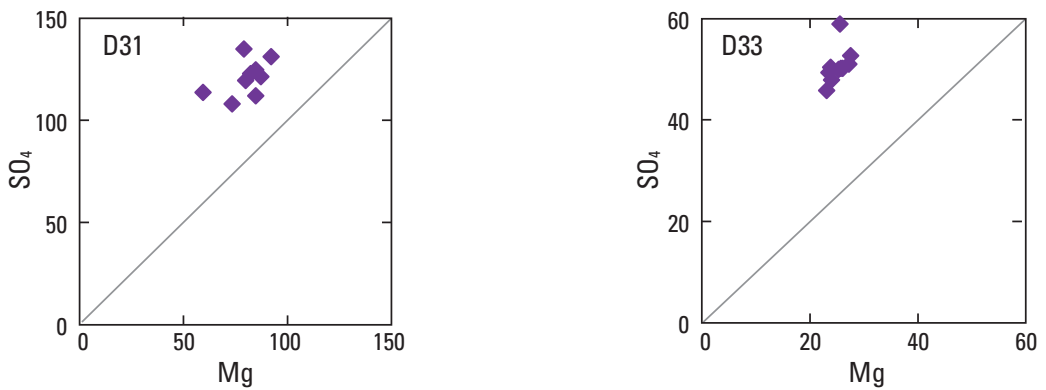

Figure 14. Ion plots showing milliequivalent ratios for $(A)$ gypsum, $(B)$ calcite, $(C)$ dolomite, $(D)$ halite, and $(E)$ epsomite (or another magnesium-sulfate mineral) from samples collected from selected monitoring wells near Deer Trail, Colorado, 1993 through 1998. [All concentrations are shown in milliequivalents per liter; diagonal line represents 1:1 concentration ratio]—Continued 
Geology also controls groundwater flow paths. Characteristics of geologic materials (bedrock and soil) such as cementation, porosity, and permeability likely determined flow paths and affected flow rates and chemistry. Characteristics of geologic materials also affect aquifer recharge and discharge locations and aquifer-mixing scenarios. The effects of flow path on water quality are discussed further in the section, Flow-Path Effects.

\section{Meteorological Effects}

Meteorology affected water quality in the study area through precipitation on surface water (wet deposition) that delivered atmospheric constituents to the water and through runoff and infiltration that transported constituents from the land surface into surface water or groundwater. The precipitation improved water quality when the chemical content of the precipitation was less concentrated than the receiving waters (dilution). The precipitation degraded water quality when the chemical content of the precipitation, runoff water, or infiltrating water was more concentrated than the receiving waters. A further effect of meteorology on water quality of the study area was the degradation of water quality by evaporation that concentrated constituents in the water during warm, dry weather.

Precipitation can affect water quality directly through wet deposition of various solutes from the atmosphere. The precipitation itself likely contained sulfate, ammonium, sodium, nitrate, chloride, and trace quantities of calcium and potassium (Nagamoto and others, 1983). Precipitation also can contain other constituents from local or regional sources. Other sources of these ions in the study area likely contributed a greater mass of these constituents to the surface and subsurface water in the study area than the direct deposition of precipitation.

Precipitation can affect water quality indirectly by mobilizing chemical constituents from the soil during runoff and infiltration. Yager and others $(2004 \mathrm{~b}$, table 10$)$ reported that aluminum, barium, calcium, iron, strontium, and sulfate would be the ions likely to be present in the largest concentration in the water leached from farmland soils in the vicinity of the study area that did not receive any biosolids applications (although some common anions such as bromide, chloride, fluoride, and nitrate were not analyzed). Few samples collected during 1993-1999 were analyzed for aluminum, barium, and strontium, but all waterquality samples were analyzed for calcium, iron, and sulfate. Calcium and sulfate were detected in all samples collected from the study area during 1993-1999, often in large concentrations (table 5). Iron was detected in all samples from some wells but was not detected in samples from most wells, likely because of the large MRL used by the laboratory for the 1993-1998 analyses (table 5). Barium, calcium, iron, strontium, and sulfate were detected in all samples that were collected in 1999 (table 7); aluminum was detected in only one sample collected in 1999 (table 7). These data indicate that meteorology could have affected water quality in the study area by mobilizing chemical constituents from the soil during runoff and infiltration. No information was available for the study area with regards to chemicals that could be mobilized from precipitation on roads, buildings, vehicles, or other objects. Chemicals that could be mobilized from precipitation on biosolids are discussed in the section, Biosolids Effects.

Meteorology can affect the water quality of the study area in other ways besides precipitation providing solutes directly or transporting solutes from other sources. In some cases, warm surface temperatures coupled with low humidity and relatively small precipitation rates can result in evapotranspiration rates that exceed precipitation-infiltration rates. Pond water evaporates, and the unsaturated zone can remain too dry for wetting fronts to migrate all the way to the saturated zone (the water table). Solutes can become concentrated in ponds, soil, and pore water. For example, pore-water samples were collected from the study-area unsaturated zone in 1994 after a relatively rainy spring period. Chloride concentration in the pore water ranged from about 27 to $1,200 \mathrm{mg} / \mathrm{L}$ in the 1.5 -ft depth and was about $140 \mathrm{mg} / \mathrm{L}$ in the single sample collected from the 3 -ft depth (table 5). Nitrate concentration in the pore water ranged from 7 to $36 \mathrm{mg} / \mathrm{L}$ in the 1.5 -ft depth and was about $30 \mathrm{mg} / \mathrm{L}$ in the single sample collected from the 3 -ft depth (table 5). According to calculations using the laboratory data, copper concentration in the pore water ranged from $150-6,000 \mu \mathrm{g} / \mathrm{L}$ in the $1.5-\mathrm{ft}$ depth and was $150 \mu \mathrm{g} / \mathrm{L}$ in the single sample collected from the 3 -ft depth (table 5); however the variation in concentration resulted mostly from the different dilution factors applied, and a similar copper concentration was reported for the blank sample. Although few unsaturatedzone data could be collected, these data indicate that surficial recharge fronts generally might have been $3-\mathrm{ft}$ deep or less in the upland farmed terraces of the study area during the spring of 1994. Rainfall was not sufficient to push these chemical recharge fronts beneath the farmed fields all the way to the water table in the locations monitored. Instead, the pore-water data indicate that reservoirs of some solutes such as chloride, nitrate, and possibly copper were present in the soil near the surface and could be transported to the aquifers through a larger wetting front or through preferential flow paths. Thus, during drier periods, much of the recharge to groundwater likely happened at focused recharge areas in stream valleys, outcrop areas, or areas that had desiccation cracks.

The degree that meteorology affected the water quality of the study area was evaluated by using a variety of information. The continuously recorded precipitation data from wells D2 and D23 during 1996 through 1998 indicate that most of the precipitation was received in the study area as rainfall in summer during July-August and sometimes in spring (Yager and Arnold, 2003). The USGS observed that some precipitation events caused runoff of water to valleys that recharged streams and ponds, which may have resulted in focused recharge of groundwater at selected locations but may not have caused a noticeable groundwater-level increase at the monitoring wells. Some precipitation events caused distributed infiltration of water to the water table and thereby recharged aquifers more directly. A comparison of precipitation data with depth to water indicates that response time of water level to precipitation varied throughout the study area; sometimes 
response was immediate and sometimes response was delayed (Yager and Arnold, 2003). A comparison of precipitation data with continuously recorded water-temperature and specificconductance data from well D6 indicates a short response time of groundwater to precipitation at that site. Groundwater recharge information, therefore, can be inferred from the monitoring of precipitation, depth to groundwater, water temperature, and specific conductance (Yager and Arnold, 2003), but also can be inferred from DG-CFC data and calculations (tables 11 and 12). Still, recharge information is available for only selected wells in the study area.

The USGS recharge information indicates that groundwater likely was recharged directly by precipitation at some (but not all) locations. The DG-CFC data from 1998 indicated sites where groundwater was recharged by rainfall, such as at well D6 (table 11). Groundwater likely was recharged by precipitation at sites where water levels increased rapidly after precipitation (for example, at least some of the water from wells D2 and D23; Yager and Arnold, 2003). Water-quality data also indicated sites where groundwater was recharged by precipitation (for example, at least some of the water from wells D6, D9, and D22). At well D6 where rapid response to precipitation was discernible from the groundwater-temperature and specific-conductance data and dissolved ions were present in groundwater at large concentrations, precipitation resulted in the dilution of major ions in groundwater (Yager and Arnold, 2003). Sulfate concentrations and specific-conductance values in water samples from wells D6 and D22 generally decreased temporarily (indicating dilution) when the aquifers recharged (Yager and Arnold, 2003). Similarly, concentrations of many constituents and specific-conductance values decreased temporarily in well D9 following rainfall. This apparent short-term dilution effect on groundwater quality is consistent with minerals in the geologic materials providing most of the solutes to groundwater rather than rainfall transporting major-ion solutes from the land surface into the aquifers during recharge. Thus, geology appears to have affected water quality in the study area more than meteorology during 1993-1998.

\section{Microbiological Effects}

Microbiological effects on water quality of the study area predominantly were the microbially mediated cycling of redox-sensitive constituents. Microorganisms are common in natural environments and include bacteria and algae present in the groundwater, surface water, and the unsaturated zone. Microbial processes such as fixation, photosynthesis, respiration, and uptake affect the cycling of constituents from one form to another and can catalyze chemical reactions such as oxidation and reduction (these reactions occur simultaneously, and the combination is called redox). In oxidation reactions, chemical compounds transfer electrons, often by gaining oxygen molecules; the transformation of ammonia to nitrite to nitrate is called nitrification and is an example of oxidation. In reduction reactions, chemical compounds gain electrons, often by losing oxygen molecules. The transformation of nitrate
Table 11. Groundwater information indicated by dissolved-gas sampling at selected wells near Deer Trail, Colorado, 1998.

[The dissolved-gas data and supporting information were reported by Yager and Arnold (2003, table II.5). Sample-site locations are shown in figure 2]

\begin{tabular}{|c|c|c|c|c|}
\hline $\begin{array}{l}\text { Sample } \\
\text { site }\end{array}$ & $\begin{array}{c}\text { Sample } \\
\text { date }\end{array}$ & $\begin{array}{l}\text { Suggested } \\
\text { recharge } \\
\text { source }\end{array}$ & $\begin{array}{c}\text { Apparent } \\
\text { denitrification } \\
\text { of groundwater }\end{array}$ & $\begin{array}{l}\text { Very reducing } \\
\text { conditions along } \\
\text { groundwater } \\
\text { flow path }\end{array}$ \\
\hline D5 & $11 / 19 / 98$ & $\begin{array}{l}\text { Pond or other } \\
\text { groundwater }\end{array}$ & Slight & No \\
\hline D5 & $11 / 19 / 98$ & $\begin{array}{l}\text { Pond or other } \\
\text { groundwater }\end{array}$ & Slight & No \\
\hline D6 & $11 / 24 / 98$ & Rain & Slight & No \\
\hline D6 & $11 / 24 / 98$ & Rain & Slight & No \\
\hline D9 & $11 / 17 / 98$ & Pond & None & No \\
\hline D10 & $11 / 19 / 98$ & Pond & Slight & No \\
\hline D13 & $11 / 18 / 98$ & $\begin{array}{l}\text { Pond or other } \\
\text { groundwater }\end{array}$ & Considerable & No \\
\hline D14 & $11 / 24 / 98$ & $\begin{array}{l}\text { Other } \\
\text { groundwater }\end{array}$ & Slight & No \\
\hline D17 & $11 / 18 / 98$ & Pond & Slight & No \\
\hline D17 & $11 / 18 / 98$ & Pond & Slight & No \\
\hline D24 & $11 / 17 / 98$ & $\begin{array}{c}\text { Other } \\
\text { groundwater }\end{array}$ & None & No \\
\hline D24 & $11 / 17 / 98$ & $\begin{array}{l}\text { Other } \\
\text { groundwater }\end{array}$ & None & No \\
\hline D25 & $11 / 18 / 98$ & Pond & Considerable & Yes \\
\hline
\end{tabular}

to nitrite to nitrogen gas is called denitrification and is an example of reduction. Decomposition of microorganisms also transforms constituents; amino acids from the dead microorganisms result in ammonia. These transformations of nitrogen are part of the nitrogen cycle. Microbial processes also affect the cycling of phosphorous, sulfur, iron, manganese, and organic matter (Stevenson, 1994; Hem, 1992; McMahon and Chapelle, 2008).

Oxygen dissolved in the water affects redox reactions. Groundwater at most sites in the study area usually was oxic (contained dissolved oxygen at concentrations equal to or greater than $0.5 \mathrm{mg} / \mathrm{L}$; McMahon and Chapelle, 2008). Dissolved-oxygen measurements for groundwater in the study area ranged from less than $0.5 \mathrm{mg} / \mathrm{L}$ to $9 \mathrm{mg} / \mathrm{L}$ (table 5); the median dissolved-oxygen value of all groundwater samples collected during 1993-1998 was $1.3 \mathrm{mg} / \mathrm{L}$. The largest dissolvedoxygen values were measured in samples from well D9, which was a low-producing well that had oxygenated groundwater and usually pumped dry or almost dry (likely introducing additional oxygen into the sampling tubing) during sampling. Accurate dissolved-oxygen measurements of groundwater are difficult to obtain because a water sample in equilibrium in the subsurface can increase in dissolved oxygen when brought up to the land surface, and certain pumping equipment used to sample groundwater also can artificially increase dissolved oxygen in the water sample even if the well is not pumped dry. Where dissolved-oxygen values were greater than about $5 \mathrm{mg} / \mathrm{L}$, the well-pumping mechanism might have been introducing atmospheric air into the sample (large air bubbles generally were 
Table 12. Groundwater information indicated by chlorofluorocarbon sampling at selected wells near Deer Trail, Colorado, 1998.

[Apparent recharge date is an estimate for the groundwater sampled in 1998. Temp., temperature; Elev., elevation; ${ }^{\circ} \mathrm{C}$, degrees Celsius; chlorofluorocarbonsampling and age-dating methods and model from Busenberg and Plummer, 1992]

\begin{tabular}{|c|c|c|c|c|c|c|c|c|c|}
\hline \multirow{2}{*}{$\begin{array}{l}\text { Sample } \\
\text { site }\end{array}$} & \multirow{2}{*}{$\begin{array}{l}\text { Sample } \\
\text { date }\end{array}$} & \multicolumn{2}{|c|}{$\begin{array}{l}\text { Estimated } \\
\text { recharge }\end{array}$} & \multirow{2}{*}{$\begin{array}{c}\text { Apparent } \\
\text { groundwater } \\
\text { recharge date, } \\
\text { as calculated } \\
\text { by the model }\end{array}$} & \multirow{2}{*}{$\begin{array}{l}\text { Certainty } \\
\text { of age }\end{array}$} & \multirow{2}{*}{$\begin{array}{c}\text { Apparent } \\
\text { mixing of old } \\
\text { and young } \\
\text { ground water }\end{array}$} & \multirow{2}{*}{$\begin{array}{c}\text { Apparent } \\
\text { denitrification }\end{array}$} & \multirow{2}{*}{$\begin{array}{c}\text { Apparent } \\
\text { effects from } \\
\text { septic or } \\
\text { wastewater }\end{array}$} & \multirow{2}{*}{$\begin{array}{c}\text { Well samples } \\
\text { represent } \\
\text { water-quality } \\
\text { effects from } \\
\text { biosolids }\end{array}$} \\
\hline & & $\begin{array}{l}\text { Temp. } \\
\left({ }^{\circ} \mathrm{C}\right)\end{array}$ & $\begin{array}{l}\text { Elev. } \\
\text { (feet) }\end{array}$ & & & & & & \\
\hline D3 & $11 / 17 / 98$ & 11.0 & 5,217 & $1970-1974$ & High & Possibly & No & Possibly & Possibly \\
\hline D5 & $11 / 19 / 98$ & 11.1 & 5,217 & 1975-1980 & High & No & No & No & No \\
\hline D6 & $11 / 24 / 98$ & 15.6 & 5,217 & $1961-1969^{1}$ & Moderate $^{1}$ & Yes $^{4}$ & Yes & No & Possibly \\
\hline D13 & $11 / 18 / 98$ & 11.0 & 5,264 & 1970-1974 & High & Slight & Yes & No & No \\
\hline D14 & $11 / 24 / 98$ & 12.3 & 5,264 & $1970-1975$ & High & No & No & No & No \\
\hline D17 & $11 / 18 / 98$ & 11.0 & 5,264 & 1964-1968 & High & Slight & No & No & No \\
\hline D24 & $11 / 17 / 98$ & 12.6 & 5,200 & 1989-1995 & Low $^{2}$ & Possibly & Yes & Possibly & Possibly \\
\hline $\mathrm{D} 25$ & $11 / 18 / 98$ & 9.9 & 5,160 & $1955-1960$ & Moderate $^{3}$ & No & Yes & No & Possibly \\
\hline
\end{tabular}

${ }^{1}$ Results indicate mixing of old groundwater, such as bedrock groundwater, with younger water. The age of the older fraction was likely older than that indicated by the model, and the age of the younger fraction was likely younger than that indicated by the model.

${ }^{2}$ Sample likely was exposed to air during pumping, which would have introduced modern levels of chlorofluorocarbons into the sample and made the sample appear more recently recharged than actual.

${ }^{3}$ Sample contained methane, which means the chlorofluorocarbon amounts likely were microbially decreased since recharge, so actual age was more recent than calculated by the model.

${ }^{4}$ Ratios of chlorofluorocarbons indicate that at least 50 percent of this water was young (post 1940).

visible in the pump tubing for these samples). Measurements of dissolved oxygen listed in table 10 (iodometric method) are more accurate than those listed in table 5 (amperometric method). Where groundwater was oxic, microorganisms will preferentially utilize dissolved oxygen in the groundwater (McMahon and Chapelle, 2008). Where groundwater was post oxic (suboxic or anoxic: dissolved-oxygen values were less than $0.5 \mathrm{mg} / \mathrm{L}$ ), microorganisms will utilize other available electron acceptors (first nitrate, then manganese, then iron, then sulfate, then carbon dioxide from organic compounds) (McMahon and Chapelle, 2008).

Microbiological processes affecting water quality of the study area are better understood from dissolved-gas, chlorofluorocarbon, and redox sampling that were incorporated into the study in 1998-1999. Dissolved gases and chlorofluorocarbons are trace constituents that are present in air, then trapped in water when it infiltrates through the subsurface. Some dissolved gases and chlorofluorocarbons can stay unchanged in the groundwater after recharge and sometimes can be used as indicators of groundwater recharge history, flow path, and microbial conditions. Dissolved-gas and chlorofluorocarbon (DG-CFC) data collected in November 1998 for the study area were reported by Yager and Arnold (2003). The dissolvedgas and chlorofluorocarbon data indicate that denitrification was common in the study area, especially at wells D13 and D25 (tables 11 and 12). Chlorofluorocarbon data indicated that denitrification had occurred in groundwater at wells D6, D9, and D24 (table 12), yet denitrification was not sufficient to prevent nitrate concentrations from increasing during 1993-1999 at these sites because the groundwater usually was oxic. Dissolved-gas data indicated only slight denitrification in groundwater from well D6 and no denitrification in groundwater from wells D9 and D24. Where denitrification is actively occurring, nitrate is reduced to nitrite and even to nitrogen gas; therefore, nitrate produced from additional nitrogen inputs to that site (such as from biosolids or other fertilizers) will be consumed in post-oxic groundwater.

The results from redox sampling can indicate which redox processes were active in the groundwater in 1999 and the stability of the intermediate-reaction product (hydrogen) and final products (such as sulfide and methane) in the groundwater (McMahon and Chapelle, 2008). The redox-indicator data and results are reported in table 10 . Only monitoring wells that had shallow, post-oxic groundwater with larger iron concentrations in historic samples could provide useful redox information and were sampled. The theory, sampling methods, and interpretations are based on the work of Chapelle and others (1995), Lovley and others (1994), and McMahon and Chapelle (2008), who used electron-acceptor (nitrate, manganese, iron, and sulfate) consumption, intermediate-product (hydrogen) concentrations, and final-product (ferrous iron, sulfide, and methane) production to determine the predominant terminal electronacceptor process (TEAP) in post-oxic groundwater. Listed in order of most efficient to least efficient metabolic process (and therefore in order of reaction), the TEAPs consist of oxygen reduction, nitrate reduction (denitrification), ferric-iron reduction, sulfate reduction, and carbon-dioxide reduction (methanogenesis) (McMahon and Chapelle, 2008). The predominant TEAPs were determined for the groundwater sites in the vicinity of the study area that were sampled for redox-sensitive constituents in July 1999 (table 10).

In general, the redox information indicates that oxygen was depleted (reducing groundwater conditions) at the redoxsampled wells in the study area in 1999 (table 10). The lack of 
hydrogen sulfide in these groundwater samples is consistent with pyrite precipitation, which can remove sulfide from the water (Chapelle and others, 1995, p. 360); pyrite was observed in the cores of most boreholes in the vicinity of the study area. Monitoring wells in the study area that were not sampled for redox-sensitive constituents had oxic-type groundwater, which means that groundwater had sufficient dissolvedoxygen concentrations to indicate that oxygen reduction was likely the predominant TEAP. Monitoring wells in the study area that were sampled for redox-sensitive constituents had post-oxic type groundwater. Groundwater from well D21 was slightly reduced compared with that of wells having oxictype water; any oxygen had been reduced so iron reduction was the predominant TEAP at this site. Groundwater from wells near wetland areas (wells D15, D16, D23, and D30) was further reduced compared with that of wells having oxic-type water; any oxygen and nitrate in this groundwater had been reduced, so iron reduction or sulfate reduction was the predominant TEAP at these sites (table 10). Groundwater data from well D22, which likely was completed in shale near the bottom of the Laramie-Fox Hills hydrostratigraphic unit (Yager and Arnold, 2003), indicated sulfate-reduction was the predominant TEAP at that site (oxygen and nitrate had been reduced). The two nonsequential TEAPs indicated by data from DTX2 and DTX10A (monitoring wells installed in 1999 for the USGS Expanded Monitoring Program near Deer Trail, shown in fig. 3) mean the groundwater may have been stratified, having a distinct zone of very reduced water and a zone of oxygenated water in the vicinity of those wells, so additional nitrate may not be reduced by the microorganisms. The redox information indicates that additional nitrate likely will be microbially reduced in groundwater at wells D15, D16, D21, D22, D23, and D30. In general, microbial processes in oxygen-depleted shallow groundwater (such as in much of the Muddy Creek area) have kept nitrate concentrations small and could reduce additional nitrogen inputs to the groundwater.

Pond formation can change redox conditions of the groundwater and, therefore, concentrations of redox-sensitive constituents (such as dissolved oxygen, nitrate, manganese, iron, and sulfate). Pond presence often resulted in microbial effects on water quality in the study area, such as increased nitrate and decreased iron concentrations that coincided with pond formation near wells D24, D25, D26, and D32, and substantially decreased nitrate and increased iron concentrations that coincided with (anthropogenic) pond removal at well D25. Ponds provided a source of oxygenated recharge water, and associated wetlands created reducing zones (as was mentioned regarding wells D15, D16, D23, and D30). In contrast, an anthropogenic pond near well D5 that was present in 1993 dried out gradually and was gone by 1997 with little discernible effect on water quality.

\section{Flow-Path Effects}

Some flow-path effects were evident from the waterquality data collected during 1993-1999. Flow paths determine where and when the water in the aquifer originates, what processes affect the water, and what properties and chemistry are characteristic of the water. Flow paths primarily are determined by geology and meteorology.

A little flow-path information was available from the water-quality data listed in table 5 . The variation in these data indicates that various distinct flow paths in the alluvial and bedrock groundwater were likely. Trilinear graphs of these data indicate that deep bedrock-aquifer groundwater generally was not chemically distinct from shallow bedrock-aquifer groundwater or alluvial-aquifer groundwater (fig. 9), so the deep bedrock groundwater likely shared a flow path with some of the shallow bedrock-aquifer and alluvial-aquifer groundwater. However, the water-quality data listed in table 5 do not provide much information about specific flow paths.

Flow path information was provided by hydrologic data from 1993-1999 and geohydrologic information from 1999, which were reported by Yager and Arnold (2003). Hydrologic data combined with regional geohydrologic information (such as from Robson, 1981) indicate that general groundwater flow in the study area likely was from the deeper parts of the Denver Basin outward toward the Denver Basin margins, from the bedrock aquifer toward the alluvial aquifer, from the alluvial aquifer toward the bedrock aquifer, or from the land surface downward through the unsaturated zone into the bedrock or alluvial aquifers, depending on the specific location in the study area. Water-level data from the study area indicate that a significant bedrock-aquifer flow path was from the deeper, confined part of the Denver Basin in the west toward the east, at least in the vicinity of well D11 a near Muddy Creek, and that Muddy Creek alluvium may have received recharge from the Laramie-Fox Hills aquifer.

The DG-CFC sampling in the study area in 1998 provided additional flow-path information. The DG-CFC information enabled apparent groundwater recharge dates and the degree of mixing of old (pre-1940) and young (post-1940) water in the aquifer to be estimated (table 12); however, the long screens (at least $10 \mathrm{ft}$ on most of the monitoring wells) can integrate water from multiple flow paths in the sample collected from each monitoring well, which increases the uncertainty of the DG-CFC results. Of the groundwater sampled in 1998 for DG-CFC, only D9 water and possibly D24 water appear to have been recharged during the 1990's (table 12). The D9 sample may have been affected by modern air if the well was pumped dry during sampling (yielding a recharge date more recent than actual), but the apparent dilution of water-quality constituents by precipitation evident from the D9 samples also indicated a recent recharge date for at least some of the D9 groundwater. Groundwater from the other wells that were sampled in 1998 appear to have been recharged during the 1950's through 1980's (table 12), which was before biosolids were applied to the study area. Ratios of the chlorofluorocarbon isotopes can indicate whether old water (pre-1940) mixed with young water at each site, as long as the isotope concentrations were in the dateable range. For the wells where DG-CFC sampling was done in 1998 (table 12), only chlorofluorocarbon data from well D6 indicated definite mixing. The ratios indicated the young fraction made up at least 50 percent 
of the groundwater mixture (table 12) in the 1998 sample; the response of groundwater temperature and specific conductance at well D6 to precipitation also indicated a recent recharge date for at least some of the D6 groundwater. DG-CFC data also indicated mixed old and young groundwater at wells D3, D9, D13, D17, and D24 in 1998 (table 12). Old groundwater was likely bedrock groundwater, or at least water from deeper parts of the bedrock aquifer. Young groundwater likely was water recharged from surficial precipitation or surface water (such as ponds), but could also have been recharge from shallow parts of the bedrock aquifer. The dissolved-gas data also enabled recharge temperatures and, therefore, recharge sources (such as pond) to be estimated (table 11). More detailed information about these estimates was reported by Yager and Arnold (2003). In short, the DG-CFC data indicated that at least some of the groundwater followed a slow flow path after recharge before traveling to wells D3, D5, D6, D13, D14, D17, and D25, but at least some of the groundwater followed a rapid flow path after recharge to travel to wells D6, D9, and D24 (table 12). More wells in the study area, particularly those with short well screens, need to be sampled for DG-CFCs to further define flow paths in the study area. Resampling of some of these same wells in the future would enable confirmation of these groundwater apparent ages and flow paths, which can change over time.

Redox information from DG-CFC sampling and redoxindicator sampling also provide groundwater flow-path information for the study area. November 1998 DG-CFC data indicated that denitrification affected groundwater at numerous places in the study area, and that groundwater in the vicinity of well D25 had very reducing conditions somewhere along the groundwater flow path (possibly when water recharged through a wetland or flowed beneath a pond) (tables 11 and 12). July 1999 data for dissolved-oxygen, sulfide, methane, hydrogen, nitrate, and sulfate concentrations were compared for wells along Muddy Creek (table 10). These data indicated that groundwater likely flowed from D21 to D16 to D23 to D15 and generally became progressively reduced. Between well D21 and D16 (possibly from the tributary alluvial aquifer near well D20) and between well D23 and D15 (possibly from the tributary alluvial aquifer near well D17), more oxygenated, sulfate-rich water entered the aquifer. These data also indicated that this flow path did not necessarily include the bedrock aquifer in the vicinity of well DTX10A in 1999. The redox data did not indicate that water in the Muddy Creek alluvial aquifer (as represented by the data from well D15) recharged the bedrock aquifer in the vicinity of well DTX10A, but redox conditions in the Muddy Creek alluvial aquifer could change over the 6 mi between well D15 and well DTX10A, the bedrock well that was sampled.

During flow through an aquifer, groundwater chemically interacts with the geologic materials and likely is affected by hydrologic and microbial processes. Therefore, groundwater chemically evolves as it follows a flow path into and through an aquifer. Geochemical modeling can be a useful tool to evaluate this chemical evolution by helping to evaluate the effects of geology, land use, fertilizer treatments, and the various aquifer processes on groundwater. Forward modeling can be used to predict water chemistry or to evaluate likely effects of chemical inputs to the geochemical aquifer system, such as from biosolids treatments (D.L. Parkhurst, U.S. Geological Survey, oral commun., March 22, 2000). Inverse modeling can be used to evaluate how groundwater at a well may have chemically evolved, including whether the groundwater evolved from rain water dissolving rock minerals, mixing between aquifers, anthropogenic chemical inputs, or from chemical reactions along the flow path (D.L. Parkhurst, U.S. Geological Survey, oral commun., March 22, 2000). Inverse modeling, in particular, could be useful in considering some geochemical questions about groundwater in the study area. For example, this modeling could provide insight into what caused the unusual water quality at well D6 and whether the bedrock aquifer was receiving water from the alluvial aquifers (flow-path information).

To use geochemical models, the appropriate data must be available. Full geochemical modeling requires detailed mineralogy, chemical analysis of the minerals, detailed chemical information for anthropogenic chemical compounds related to land use or fertilizer treatments, and accurate, detailed waterquality data for geochemical constituents (major-ion plus comprehensive trace-element analyses). However, this study was not planned to include geochemical modeling as a primary tool, so much of the data needed for detailed geochemical modeling of the study area were not available. Data for mineralogy, mineral chemistry, and anthropogenic-compound chemistry were not collected during 1993-1999, and lithologic data for specific wells were limited. Water-quality data collected for the study area did not routinely include silica, aluminum, arsenic, boron, bromine, cobalt, fluorine, lithium, selenium, and strontium. Also, the trace-element data obtained from routine analyses were not all appropriate for geochemical modeling because all or most of the data were censored below the MRL for some of these constituents.

Despite these data deficiencies, the water-quality data obtained through the NWQL analyses of selected replicate samples, data from the USGS Expanded Monitoring Program near Deer Trail (Stevens and others, 2003), and knowledge of the geology of the area enabled some limited geochemical modeling of the study area that provided useful information. Lignite, a type of coal, was mined from the Laramie Formation (Soister, 1974) and was known to be present in the vicinity of the study area as thin, discontinuous layers in the bedrock or as weathered alluvial material that may or may not be present at any specific well. Alluvium in the study area consisted of glacial loess and outwash (minerals likely included montmorillonite, quartz, feldspar, biotite, pyroxene) and weathered conglomerates, sandstones, siltstones, and shale (minerals likely included montmorillonite, quartz, feldspar, gypsum, and calcite) (B.F. Jones, U.S. Geological Survey, oral commun., March 22, 2000). Bedrock in the study area consisted of marine sandstone, siltstone, and shale (minerals likely included montmorillonite, pyrite, quartz, carbonates, and possibly aragonite) (B.F. Jones, U.S. Geological Survey, oral commun., March 22, 2000). Montmorillonite is a common 
clay mineral, and quartz is a common mineral comprising sand grains. Using this assumed mineralogy with water-quality data from wells D5, D6, D10, D29, and D32, geochemical modeling was done with NETPATH (Plummer and others, 1994) to determine if groundwater from D6 could be chemically evolved from that of well D29 (bedrock groundwater), from upgradient shallow groundwater at D5, D10 or D32, from coal beds (if they were present at D6, which was not known), or from biosolids applied near D6 (modeled as organic matter, $\mathrm{CH}_{2} \mathrm{O}$ ). Results from this geochemical modeling indicated that groundwater in samples from well D6 likely was not evolved from a combination of groundwater from wells D5, D10, D29, or D32, but could have been evolved from rain water reacting with rocks and either the presence of coal or organic matter (such as biosolids). Thus, this preliminary geochemical modeling indicated that the groundwater flow path for well D6 likely did not include the bedrock aquifer water (as represented by well D29) or groundwater from the upgradient shallow wells, but could include bedrock groundwater from a different location or simply rain water that interacted with rocks and either coal or organic matter (representing biosolids) in the vicinity of the well. Collection of additional data for lithology, mineralogy, mineral chemistry, fertilizer chemistry, and water quality (including silica, strontium, boron, and uranium and using analytical methods that had lower MRLs) could be useful to further define study-area flow paths, especially for wells D2, D6, D9, D24, and D25.

\section{Biosolids Effects}

Biosolids were applied to the study area intermittently and at various application rates during 1993-1999 (table 1). Application records were not detailed with respect to the exact date of application to a specific area (such as a drainage basin or the area around a well) within each half-section (table 1; fig. 5). Applications listed in table 1 did not always encompass all the land within the listed half-section. Therefore, effects from biosolids on water quality were challenging to identify. To evaluate whether biosolids applications affected the water-quality of the study area during 1994 through 1998, post-biosolids-application data were compared with baseline data, water-quality data from the biosolids-treated area were compared with a control site, temporal patterns in selected water-quality constituents were examined for response to nearby biosolids applications, and other indicators of biosolids effects were considered.

\section{Comparison with Baseline Data}

If baseline data are collected from a study area before a treatment begins, as well as after the treatment, the data often are used to indicate treatment effects. However, the difference between groundwater baseline data and later data for the same site may not represent effects from a particular treatment during that time (such as farming with biosolids applied as a fertilizer) if water infiltrating through biosolids-treated soils has not yet recharged into the aquifer. Groundwater recharge can take as little as hours to as long as hundreds of years, depending on geologic and hydrologic conditions at a particular site. Yager and Arnold (2003) reported groundwater recharge times ranging from a few hours to more than 50 years in the study area. Apparent recharge dates determined from DG-CFC data for groundwater sampled from selected wells in 1998 are listed in table 12.

Regardless of whether groundwater had recharged through biosolids-treated fields when it was sampled during 1994 through 1999, the baseline data collected from the study area in 1993 were adequate to indicate that sources of major ions, nutrients, trace elements, and bacteria affected groundwater and surface-water concentrations before biosolids were applied in the study area. For example, chloride, magnesium, sodium, and sulfate concentrations were relatively large in samples from well D6 before biosolids applications began (table 5). Zinc concentration was relatively large in samples from well D12 before biosolids were applied, although the zinc concentration could be related to the pump, cables, and grease that had been in this livestock well before it was used only for monitoring. In addition, fecal streptococcus bacteria were detected in baseline samples from wells D3 and D12. Fecal coliform and fecal streptococci bacteria in water originate from animal feces, as well as from human sewage (Murray and others, 2001).

Chloride, sulfate, nitrate, and manganese concentration in some baseline groundwater samples exceeded Colorado regulatory standards. Chloride in baseline samples from well D6 exceeded the Colorado drinking-water standard. Sulfate in baseline samples from all wells except G2 exceeded the Colorado drinking-water standard (tables 5 and 8). Nitrate concentrations were relatively large and exceeded the Colorado Drinking Water standard in samples from well D9, and many wells had detectable nitrate concentrations before biosolids applications began (tables 5 and 8). Manganese concentration was relatively large and exceeded the Colorado drinking-water and agricultural standards in samples from wells D3, D6, D7, D8, and D12 before biosolids applications began (tables 5 and 8 ).

Most chemical-constituent concentrations were similar in the baseline samples as in later post-biosolids-application samples for 1994-1998 (fig. 15). Some concentration values of chloride, sulfate, nitrate, phosphate, cadmium, iron, and manganese were larger in the post-biosolids-application groundwater samples than in the baseline samples, but the median chloride, nitrate, and orthophosphate concentrations in groundwater were less in the post-biosolids-applications samples than the median of the baseline samples when data from all the monitoring wells were pooled. The smaller median concentrations in post-biosolids-application samples do not indicate that biosolids applications improved water quality in the study area. Rather, the differences between baseline and post-biosolids-application samples likely were because not all sample sites included in the post-biosolids-application sampling were available for baseline sampling, so the baseline data did not represent the full range of water-quality concentrations found in the study area. Most of the large range 

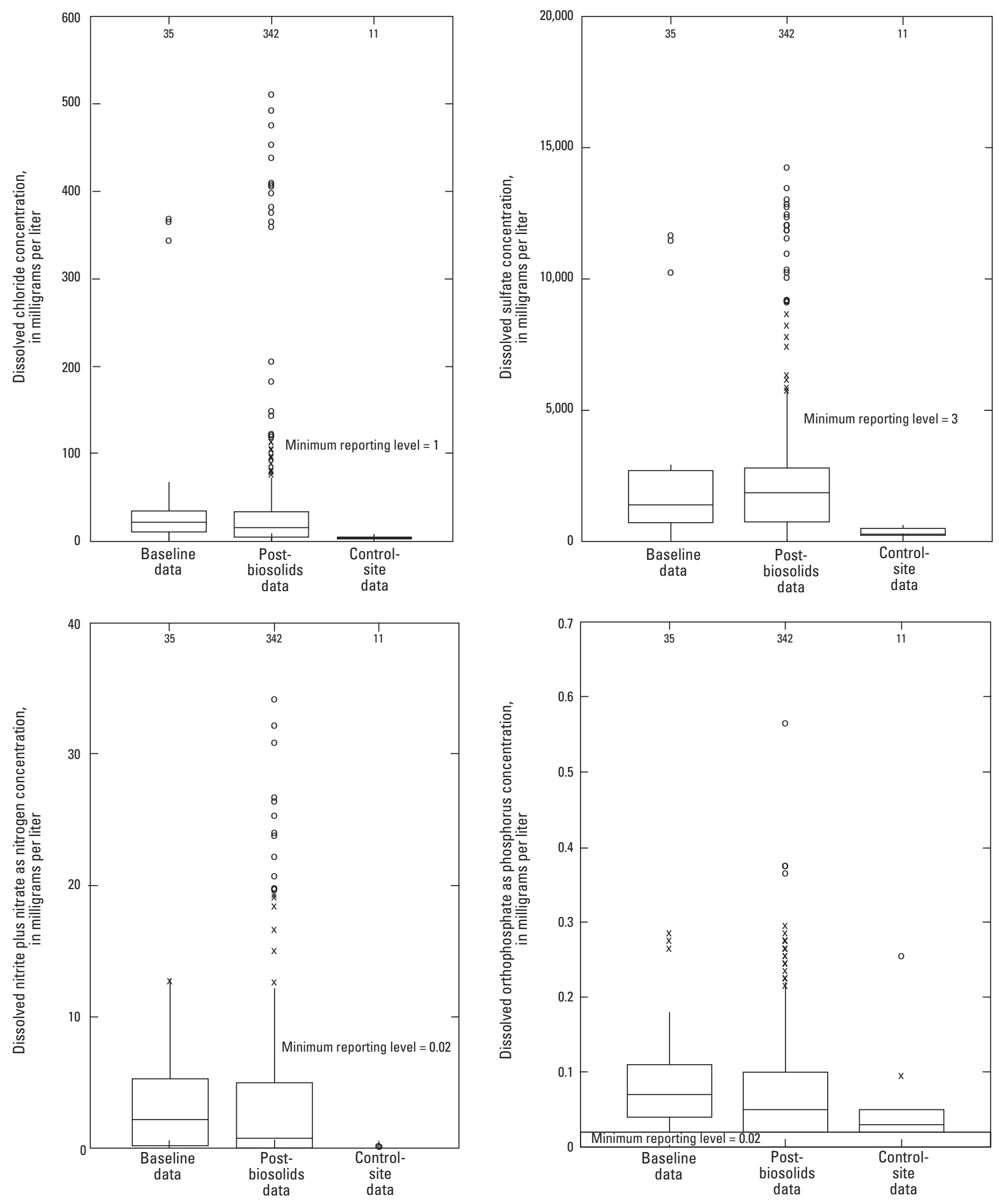

Figure 15. Comparison of baseline, post-biosolids, and control-site groundwater-quality data for selected constituents in samples from all monitoring wells near Deer Trail, Colorado, 1993 through 1998. 

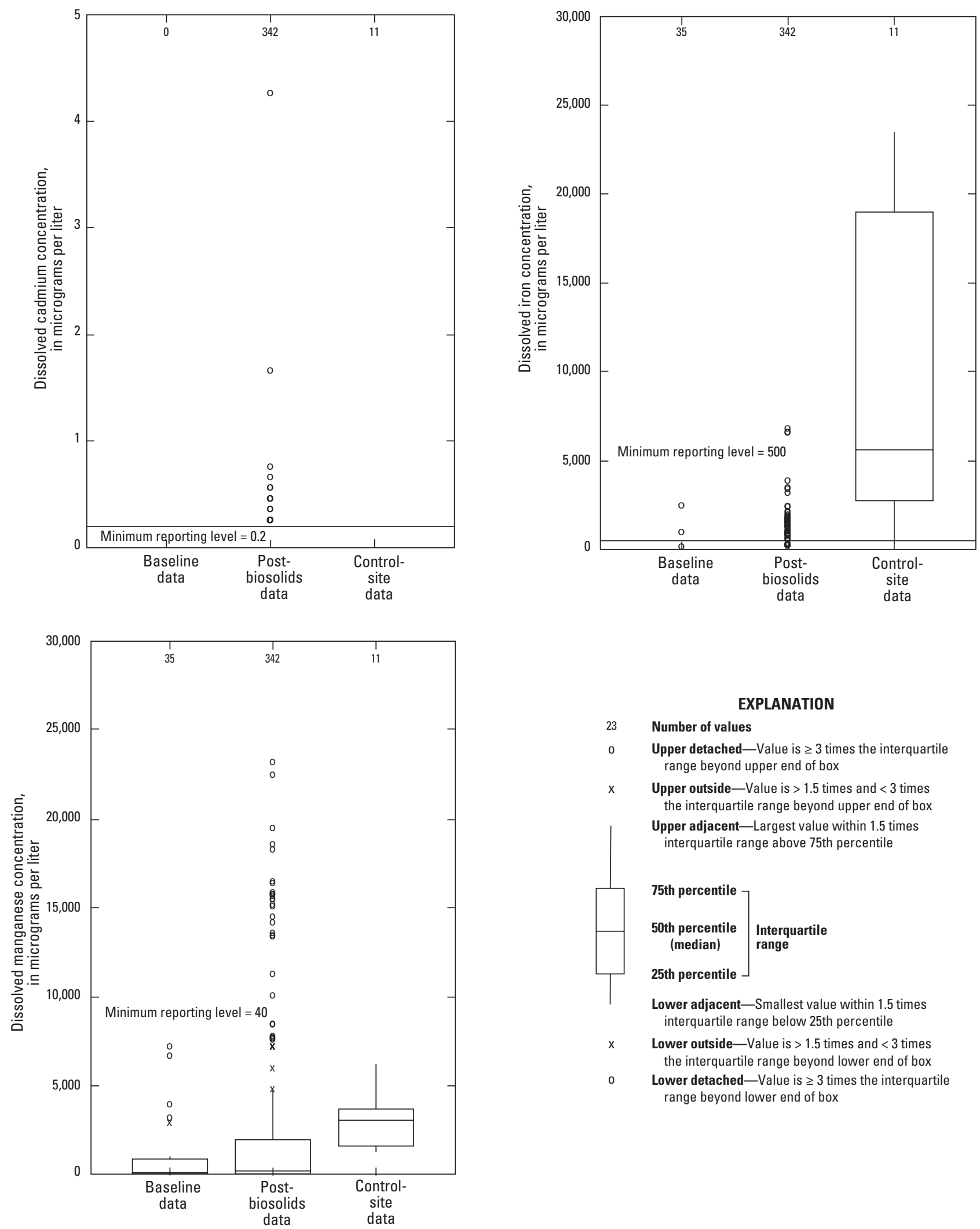

EXPLANATION

$23 \quad$ Number of values

o Upper detached-Value is $\geq 3$ times the interquartile range beyond upper end of box

$x \quad$ Upper outside-Value is $>1.5$ times and $<3$ times the interquartile range beyond upper end of box

Upper adjacent-Largest value within 1.5 times interquartile range above 75th percentile

50th percentile Interquartile

(median) range

25th percentile

Lower adjacent-Smallest value within 1.5 times interquartile range below 25 th percentile

$x \quad$ Lower outside-Value is $>1.5$ times and $<3$ times the interquartile range beyond lower end of box

o Lower detached-Value is $\geq 3$ times the interquartile range beyond lower end of box

Figure 15. Comparison of baseline, post-biosolids, and control-site groundwater-quality data for selected constituents in samples from all monitoring wells near Deer Trail, Colorado, 1993 through 1998._Continued 
in concentration shown in figure 15 for both baseline and post-biosolids-application data resulted from spatial variability (compare fig. 15 with table 5, and note that water-quality variations across the study area were discussed in the Spatial Variability section). Because of the large spatial and temporal variability in water quality for both the baseline and the postapplication data and the similarity in concentrations before and after biosolids application, effects from biosolids applications were not discernible from a simple comparison of baseline data with post-application data.

Yager and others (2004b) and Yager and McMahon (2012) determined that biosolids samples from the Metro District leached substantial concentrations of chloride, potassium, sodium, sulfate, ammonia, organic nitrogen, and phosphorus in addition to lesser amounts of many trace elements (especially iron, aluminum, molybdenum, and boron). However, soil from the vicinity of the study area that did not receive biosolids applications also leached major ions, nutrients, and trace elements (Yager and others, 2004b). Ions that had concentrations at least one order of magnitude (a factor of 10) larger in the biosolids leachate than in the soil leachate were considered biosolids-signature constituents to indicate collectively that aqueous media like groundwater and surface water had been affected by biosolids applications. Inorganic biosolids-signature constituents for aqueous media as refined from the various leachate experiments included primarily nitrogen, phosphorus, molybdenum, and tungsten, and to a lesser extent, antimony, cadmium, cobalt, copper, nickel, selenium, vanadium, and zinc (Yager and others, 2004b, table 10; Yager and McMahon, 2012). Of these, only nitrogen, phosphorus (orthophosphate as phosphorus), cadmium, copper, nickel, and zinc were analyzed routinely in both the baseline and post-biosolids-application water-quality samples. For the sites where baseline samples were collected (fig. 8), post-biosolidsapplication concentrations generally were not larger than baseline concentrations of ammonia plus organic nitrogen, phosphorus (orthophosphate as phosphorus), cadmium, copper, nickel, or zinc (table 5). Most post-biosolids-application concentrations were larger than baseline concentrations of nitrate in samples from wells D2, D3, D5, D6, D7, D9, D10 and D12. Nitrification can transform ammonia and organic nitrogen into nitrate. Therefore, the baseline data indicated that the slight increase in nitrate in shallow Muddy Creek drainage-basin groundwater and the more marked increase in nitrate in the Cottonwood Creek drainage-basin groundwater (fig. 12) could relate to biosolids applications to the study area.

In general, the comparison of baseline water-quality data with post-biosolids-applications data indicated that biosolids applications during 1993-1998 did not have a large effect on study-area water quality during 1994 through 1999; however, the baseline sampling sites were not fully representative of water quality in all parts of the study area. The baseline data indicated that biosolids effects on study-area water quality during 1994-1999 seemed to be limited to increases in nitrate concentrations. Because water affected by biosolids applications is unlikely to have recharged groundwater at all sampled locations by 1998-1999, the collection of additional water-quality data for future comparison with baseline data would be helpful in evaluating effects of biosolids on water quality of this study area.

\section{Comparison with Data from a Control Site}

Comparison of water quality at control sites (which receive no treatment) with water quality at sites affected by a treatment can indicate effects of that treatment if the control sites are representative of the study area and sufficient time is allowed for the treatment to have an effect. To evaluate the effects on water quality of a treatment such as farming with biosolids applied as a fertilizer, control sites can be useful. Because of topographic controls (fig. 2) and the lack of shallow groundwater or any flowing surface water throughout most of the study area during 1993-1998, only one control site was available: well D21 in the southwest part of the study area. This location was both topographically and hydraulically upgradient of nearly all biosolids-application areas. Well D21 also was upgradient from most of the nearby farmed areas but downgradient from grazing land. Unfortunately, access to well D21 turned out to be intermittent so samples could not be obtained from the control site every trip.

The water quality at this control site (well D21) was not better, and in fact was in some ways worse, than the water quality at the sites in the biosolids-treatment area (table 5) during 1994-1999. Concentrations of ammonia plus organic nitrogen in samples from the control site were the largest measured in the study area (maximum was $3.6 \mathrm{mg} / \mathrm{L}$ as $\mathrm{N}$ in July 1995). The laboratory reported detections of nitrite in 5 of the 11 control-site samples, although nitrate was not detected in these same samples. Most of the nitrogen in the groundwater samples from the control site was in the form of ammonia, except in July of 1994 and 1998 when most of the nitrogen was organic (table 5). Nitrogen at other sites in the study area usually was in the form of nitrate, even in baseline samples (table 5). Sulfate concentrations in control-site samples were relatively small, but not as small as those from some wells in the biosolids-treatment area (D11a, D17, D19, D24, and D26). Concentrations of other major ions and some trace elements in control-site samples were similar to those in samples from wells in the biosolids-treatment area. However, concentrations of iron and manganese were much higher in the control-site samples than in samples from most of the other monitoring wells in the biosolids-treatment area (fig. 15 and table 5). Concentrations of iron in samples from well D21 were the highest measured in the study area and fluctuated more than in samples from most of the other monitoring wells (fig. 15 and table 5). Fecal coliform bacteria were not detected in samples from the control site, but fecal streptococcus bacteria were detected in one of four samples from the control site and were detected at a similar level found in many samples from the biosolids-treated area (table 6).

More information about water quality at this control site (well D21) was obtained when the well was sampled for redox-sensitive constituents during July 1999. The groundwater sample from the control site was analyzed for additional 
water-quality constituents, including most of the inorganic biosolids-signature constituents (table 7). Phosphorus, cobalt, copper, molybdenum, nickel, and zinc were detected in the sample from the control site in 1999 at small concentrations (table 7). This sample also had smaller concentrations of ammonia and organic nitrogen than in 1994-1998 but still no detectable nitrate (table 7). The predominant TEAP at the control site that was indicated by the July 1999 sample was $\mathrm{Fe}^{3+}$ reduction; any additional nitrate in groundwater at this site was likely to be reduced (table 10). Where nitrate, $\mathrm{Fe}^{3+}$, or sulfate reduction was the predominant TEAP, nitrate concentrations in the groundwater likely would remain small, even if the groundwater was located in the biosolids-treatment area.

The single control site available for this study area was of marginal use in evaluating biosolids effects on water quality for 1993-1999. The water-quality data obtained for the study area for 1993-1999 indicate that the control site was not representative of all the other groundwater locations in the study area before biosolids applications began (compare baseline data to control-site data in fig. 15), particularly with respect to physical properties and concentrations of major ions, nitrogen, iron, and manganese. Therefore, differences in groundwater physical properties and concentrations of major ions, nitrogen, iron, and manganese between the control site and the biosolids-treatment area should not be attributed to biosolids but to geological and microbiological effects on water quality. It is interesting to note that the limited data from the July 1999 groundwater sampling indicated smaller phosphorus concentrations and larger concentrations of molybdenum, cobalt, chromium, copper, and nickel in the five samples from the biosolids-treatment area compared to concentrations in samples from the control site (table 7). Larger concentrations of biosolids-signature elements like phosphorus, molybdenum, and tungsten (as well as nitrogen, antimony, cadmium, cobalt, copper, nickel, selenium, vanadium, and zinc) in groundwater from the biosolids-treatment area relative to concentrations in samples from the control site were more likely to represent effects from biosolids applications, particularly once enough time had elapsed that the groundwater had recharged after biosolids applications began. Additional samples would need to be collected and analyzed for the biosolids-signature elements to determine if concentrations were significantly larger in the biosolids-treatment area relative to concentration variability. However, the control-site data were useful to indicate yet another variation in water quality in the study area, and may be useful (especially the 1999 data) for future data comparisons to establish biosolids effects on water quality.

\section{Comparison of Temporal Patterns}

Temporal change in water quality often is considered an indicator of treatment effects if concentrations of constituents increase during or after the time of treatment; however, temporal patterns in water quality can have other causes than the treatment. The difference in groundwater time-series data for the study area may not represent effects from the treatment (biosolids applied as a fertilizer) if water infiltrating through biosolids-treated soils had not yet recharged into the aquifer at the time samples were collected. Despite the uncertainty contributed by mixing along the long well screens, the DG-CFC results indicated that groundwater in at least some parts of the study area likely had not yet recharged through biosolids-treated areas when sampled during 1994-1998. Data from the study area also indicated that at least some of the groundwater in other parts of the study area had recharged through biosolidstreated areas; however, temporal patterns in water quality that are caused by biosolids applications could be obscured by larger effects from other processes or sources, such as natural geochemical or microbiological processes or historic land uses of the study area (prior to 1993). Also, differences in constituent concentration over time can result from sample collection, processing, or analysis and be unrelated to the study area or the treatment. Therefore, caution should be used in interpreting time-series data with respect to treatment effects. More evidence than an increase in a constituent over time is needed to indicate that biosolids applications have affected water quality.

Biosolids applications could increase bacteria colonies and concentrations of nutrients, major ions, and trace elements in study-area water. In particular, an increase in concentrations of the biosolids-signature elements that followed biosolids applications might be attributed to biosolids effects on water quality. A time lag between application dates and groundwaterconcentration increases would be expected because of the time it takes to recharge groundwater, and this time lag could be unique and vary for each site that was sampled. Increases in concentrations of biosolids-signature constituents in studyarea water samples that were caused by biosolids applications likely would be widespread and occur at some relatively consistent interval after biosolids applications. To further evaluate the effect of biosolids on water quality of the study area, concentrations of selected biosolids-signature constituents (included in table 5) and other constituents that could come from biosolids (such as fecal streptococcus bacteria; table 6) were compared to biosolids-application loads and dates (table 1). Biosolids-applications and concentrations of selected biosolids-related constituents are compared in figure 16 for selected sites that likely had at least some modern groundwater (wells D2, D6, D9, D23, and D24) and for one site that had at least some old groundwater but also had the occasional large nitrate concentration (well D25). Because copper, nickel, and zinc detections in the samples from these sites were rare, these constituents were not included in figure 16.

Biosolids were applied in the eastern part of the study area (in the vicinity of Cottonwood Creek and wells D2 and D6; DC 301 and DC 310) in December 1993 through June 1994 and in September 1997 through February 1998 (table 1; fig. 16). Nitrate concentration in samples from well D2 spiked slightly in May 1995 and then increased gradually. Nitrate concentration in samples from well D6 did not spike but did increase gradually. Chloride concentration remained fairly constant in samples from well D2, whereas chloride concentration was constant with one decrease in 1996 in samples from well D6. Cadmium concentration was largest in October 1994 but barely 

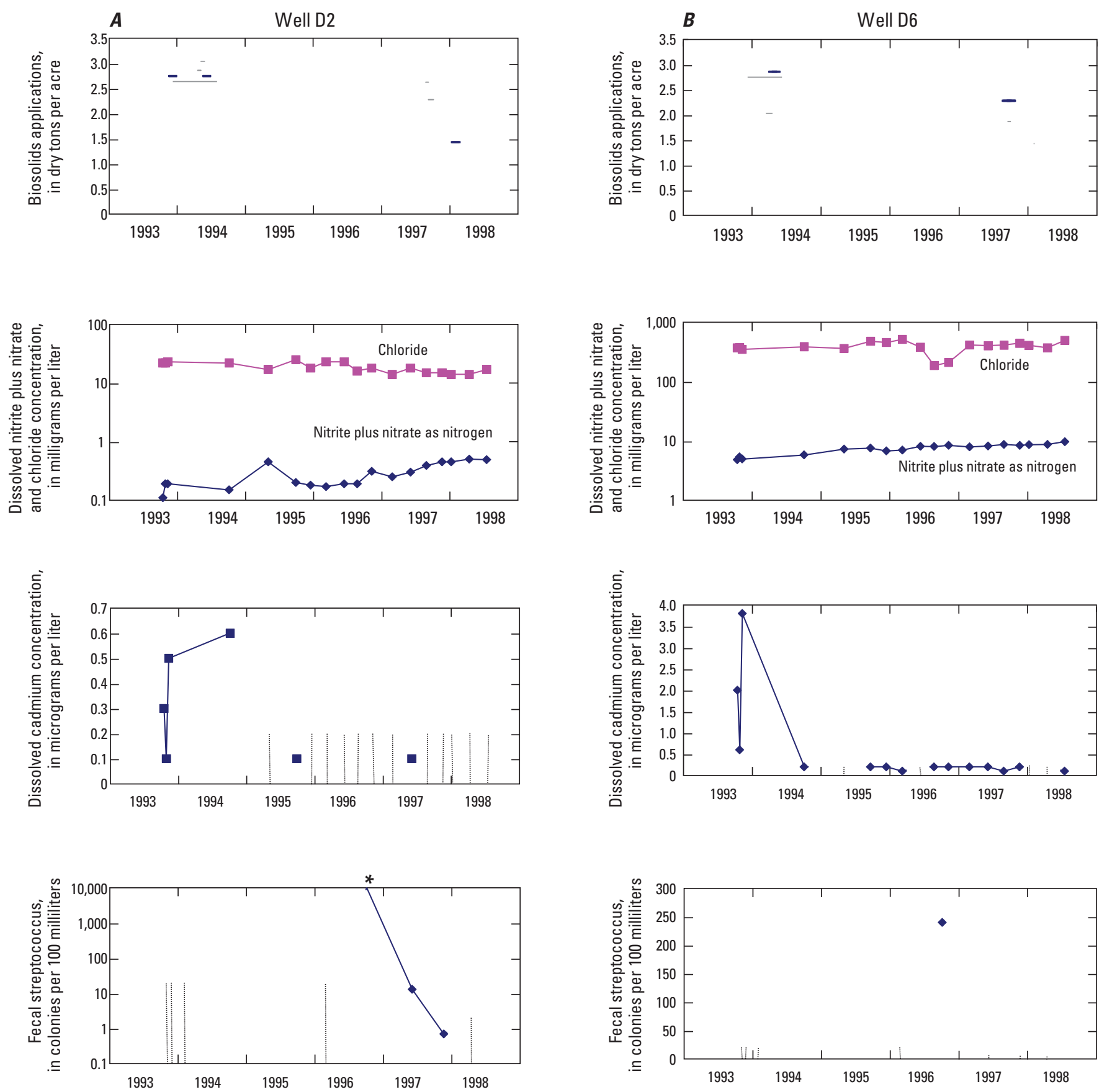

EXPLANATION

- Biosolids application to the half section (destination code) that contains the well or, if well is close to a boundary between two destination codes, biosolids application to either of the two destination codes that surround the well

- Biosolids application to a more distant upgradient destination code in the same drainage basin that contains the well

I Data that were reported by the laboratory as less than the minimum reporting level; height of dotted line corresponds to censored interval

* Reported by laboratory as $>\mathbf{1 0 , 0 0 0}$ but value may be from field or laboratory contamination

Figure 16. Comparison of selected water-quality constituents with biosolids-application data for selected sites near Deer Trail, Colorado, 1993 through 1998. 
C
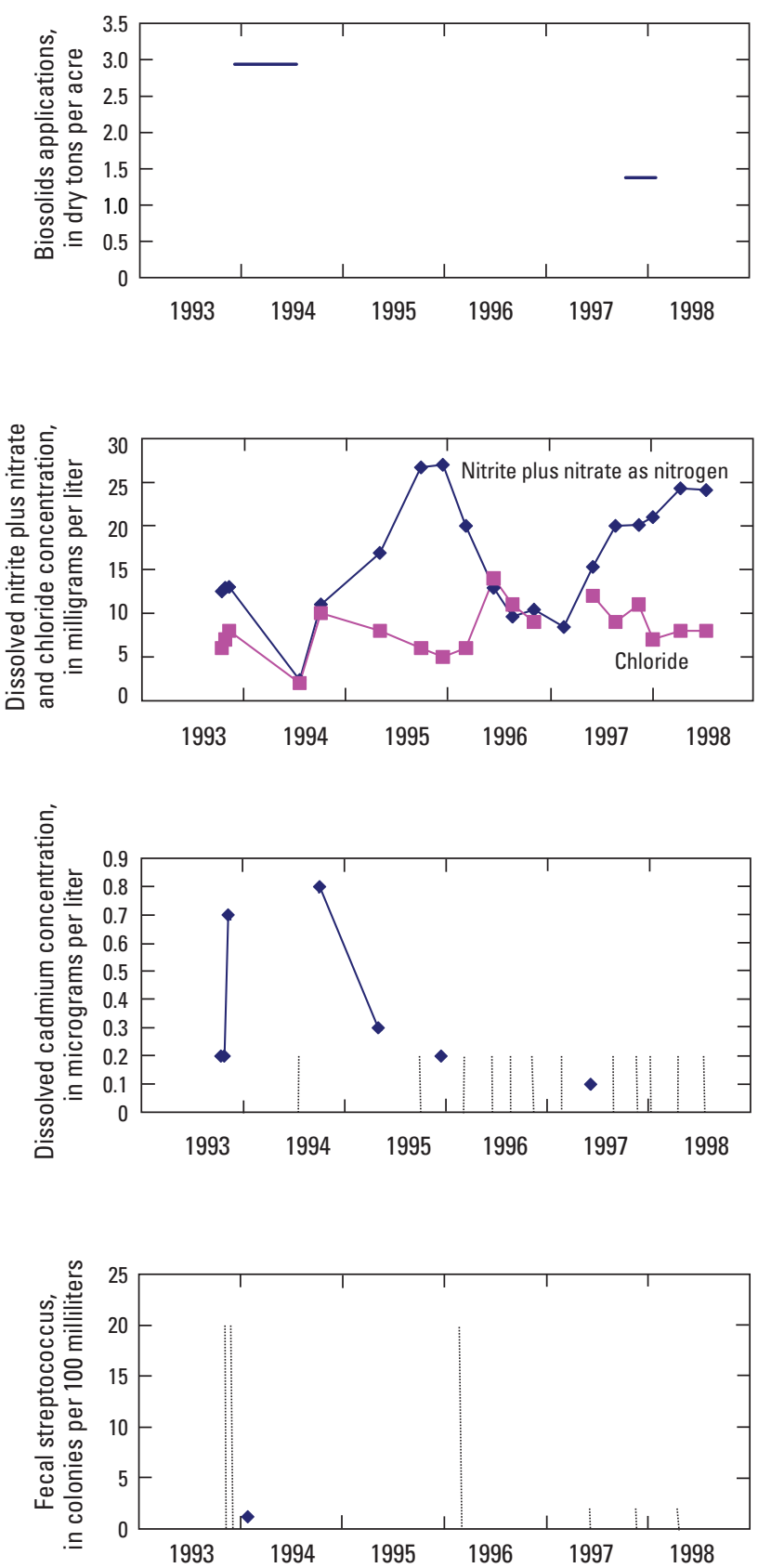

D

Well D23
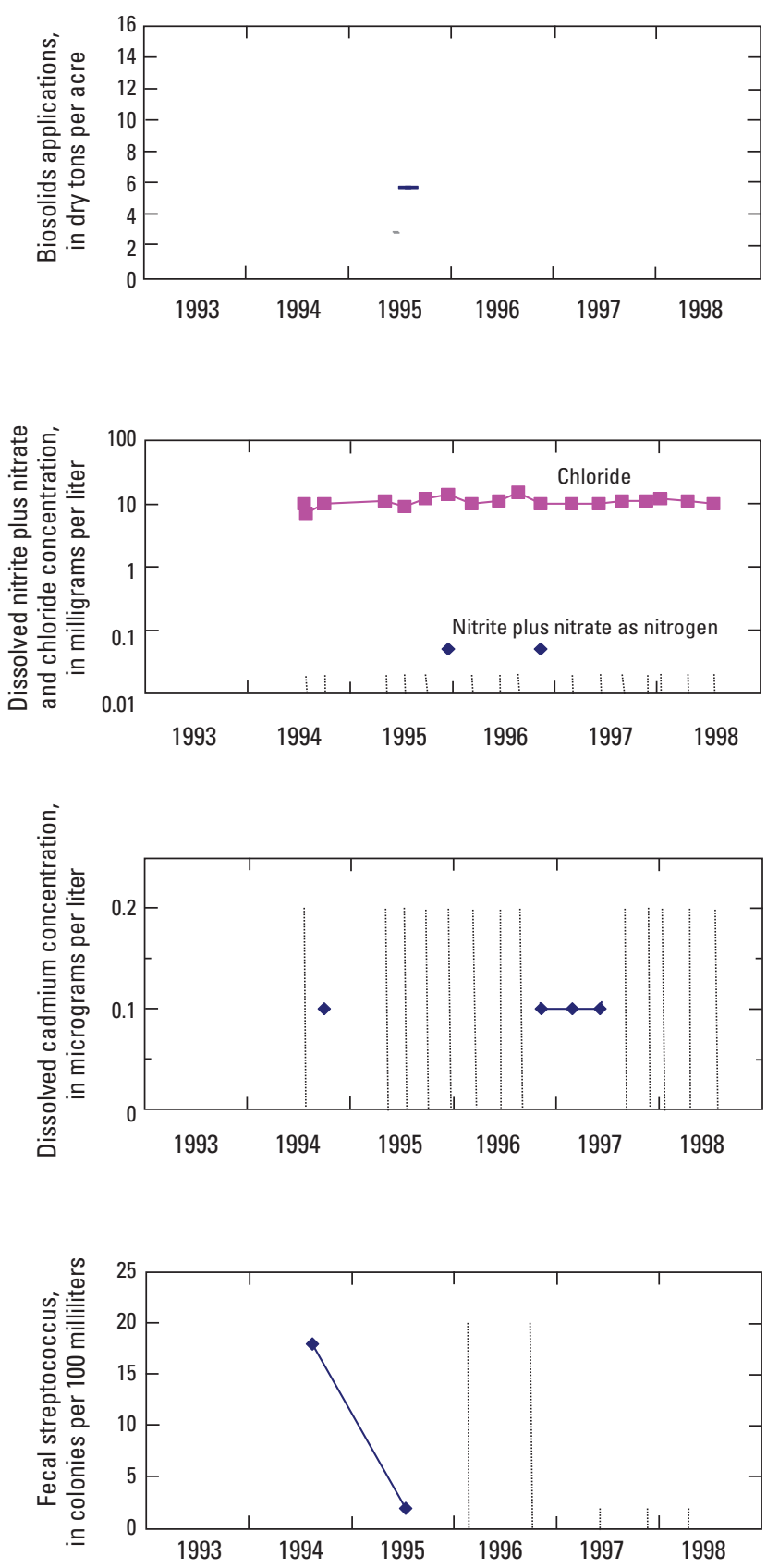

\section{EXPLANATION}

- Biosolids application to the half section (destination code) that contains the well or, if well is close to a boundary between two destination codes, biosolids application to either of the two destination codes that surround the well

Biosolids application to a more distant upgradient destination code in the same drainage basin that contains the well

Data that were reported by the laboratory as less than the minimum reporting level; height of dotted line corresponds to censored interval

Figure 16. Comparison of selected water-quality constituents with biosolids-application data for selected sites near Deer Trail, Colorado, 1993 through 1998.-Continued 
$E$
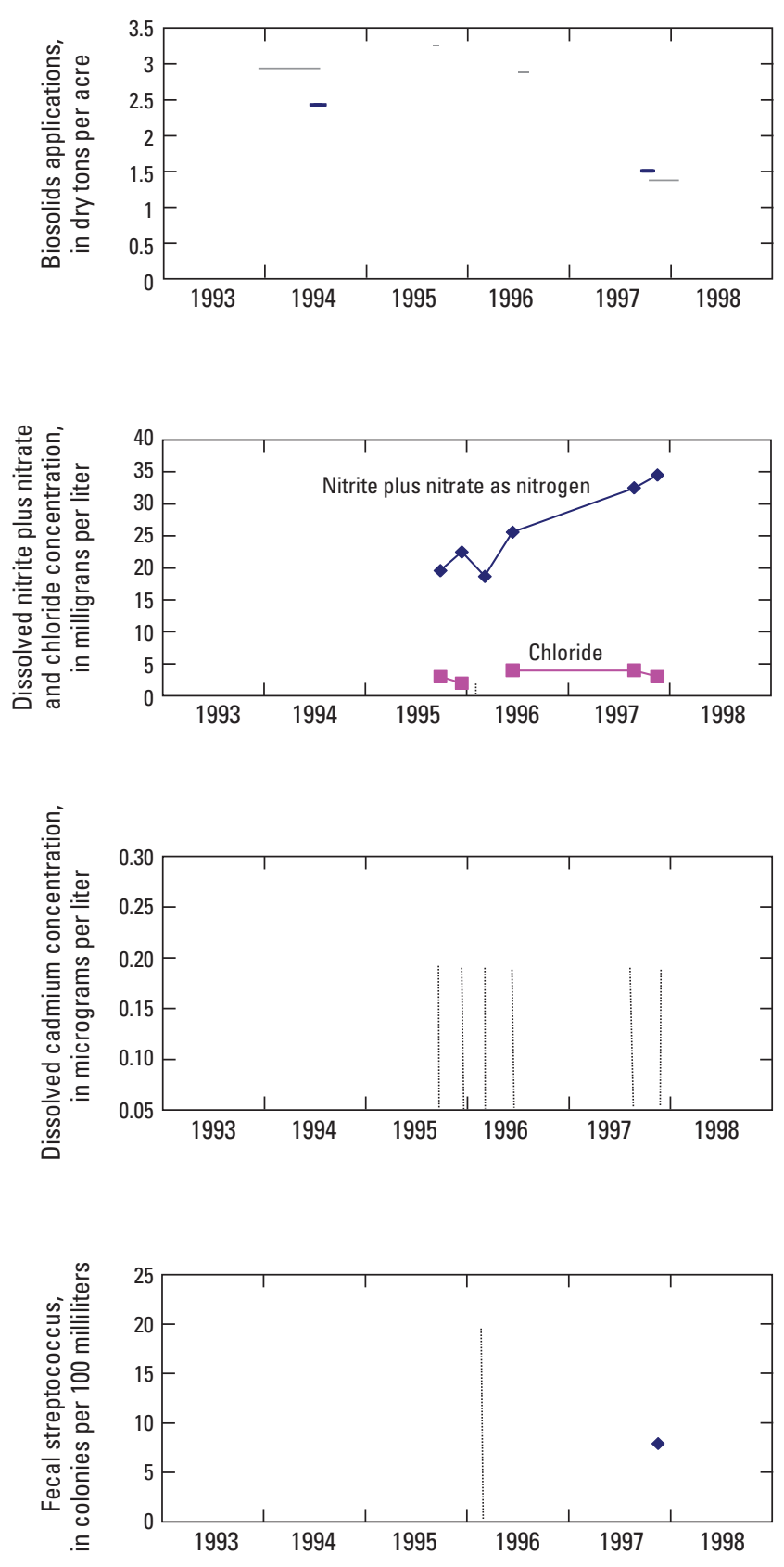

$\boldsymbol{F}$

Well D25
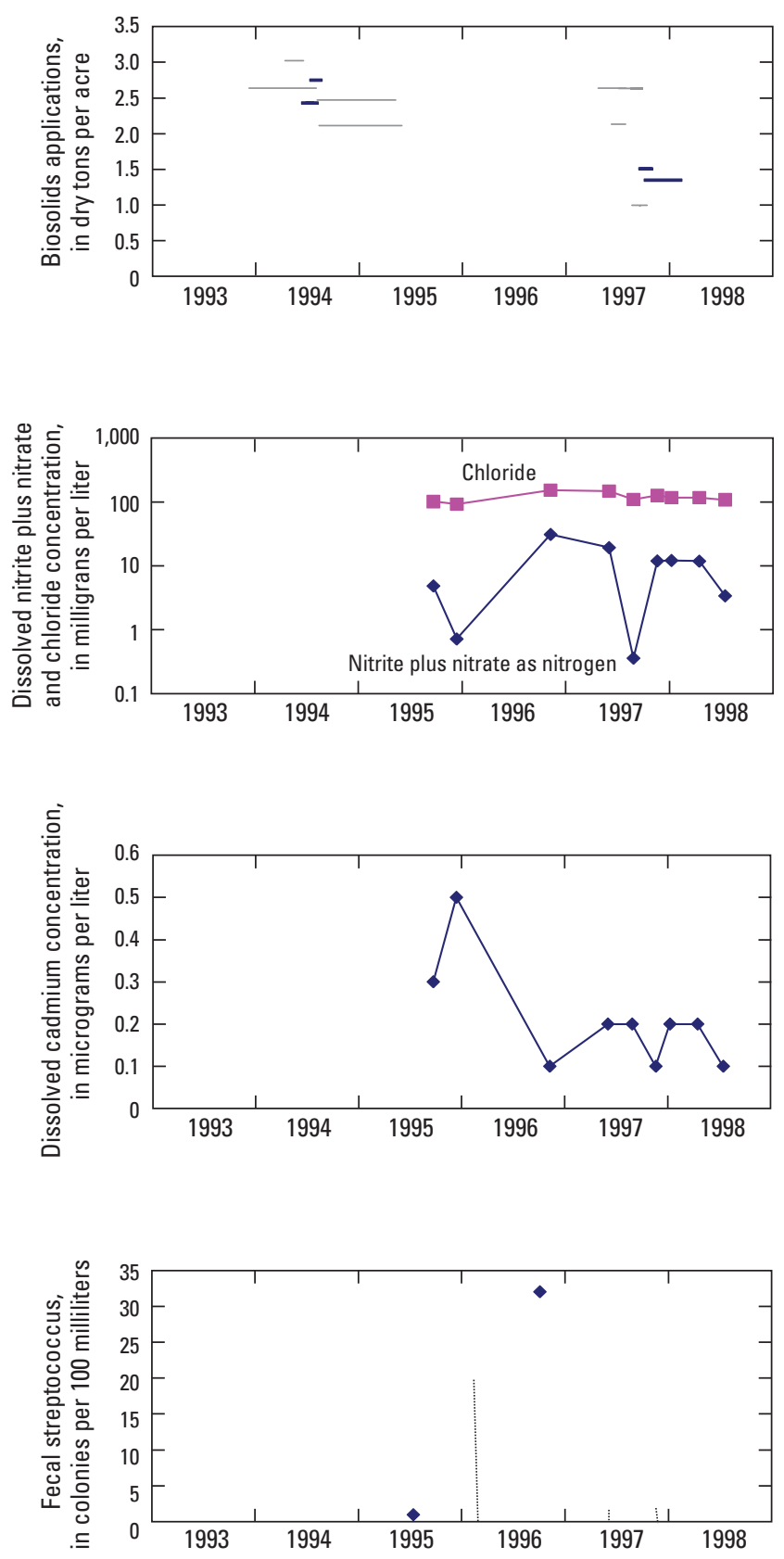

EXPLANATION

- Biosolids application to the half section (destination code) that contains the well or, if well is close to a boundary between two destination codes, biosolids application to either of the two destination codes that surround the well

Biosolids application to a more distant upgradient destination code in the same drainage basin that contains the well

Data that were reported by the laboratory as less than the minimum reporting level; height of dotted line corresponds to censored interval

Figure 16. Comparison of selected water-quality constituents with biosolids-application data for selected sites near Deer Trail, Colorado, 1993 through 1998.-Continued 
larger than baseline concentrations in samples from well D2, whereas cadmium concentration was not detected above the MRL except for baseline concentrations in samples from well D6. Fecal streptococcus bacteria were not detected in samples from well D2 until October 1996 but then were detected in the highest number cultured for study-area samples (greater than 10,000 colonies per $100 \mathrm{~mL}$ ) before decreasing throughout 1997. Fecal streptococcus bacteria also were detected in numbers much higher than the MRL in the sample from well D6 in October 1996. Bacteria colonies were detected in other samples from some of the Cottonwood Creek drainage-basin wells and a pond, but not consistently or in increasing quantities. Phosphorus concentration spiked slightly in the September 1995 sample from well D2 but stayed near baseline concentrations in samples from well D6. Copper and nickel concentrations spiked slightly higher than baseline concentrations in the June 1997 samples from wells D2 and D6. The generally increasing concentrations of nitrate in samples from wells D2 and D6 may have resulted from biosolids applications in the area, but the rather random temporal patterns in the other water-quality data did not indicate much effect of biosolids applications on water quality during 1993-1998.

Biosolids were applied in the vicinity of well D23 (DC 326 and DC 328) only during June-August 1995 (table 1; fig. 16). The July 1995 ammonia concentration in the sample from well D23 increased slightly, but concentrations of chloride, other nutrients, cadmium, copper, nickel, and zinc stayed about the same, were not detected, or were detected at about the same concentration as the MRL. Detections of fecal streptococcus bacteria were about the same as the MRL, and the number of bacteria decreased in the July 1995 sample relative to the August 1994 sample. Therefore, the temporal data pattern for well D23 did not indicate that the biosolids application in the vicinity of well D23 affected water quality during 1994-1998.

Biosolids were applied to the north part of the study area in the vicinity of wells D9 and D24 (DC 302 and DC 304) in December 1993 through July 1994 and October 1997 through January 1998 (table 1; fig. 16). Nitrate concentrations increased in samples from well D9 within one year of biosolids application, decreased, and then began increasing again without additional biosolids application. Chloride concentration varied differently from nitrate concentration but generally increased during 1993-1998 in samples from well D9. Cadmium concentration in samples from well D9 fluctuated most in November 1993 through September 1995 samples then was fairly constant. The single fecal streptococcus bacteria detection in the January 1994 sample from well D9 (during the biosolids application period) was smaller than that of the control site and the MRL. The largest phosphorus concentration reported for samples from well D9 was for the July 1994 sample. Copper concentration in samples from well D9 was not detected above the MRL (table 5). The only concentrations of nickel detected in samples from well D9 were in March 1996, November 1997, and January 1998, which did not correspond to the dates of zinc detections (October 1994 and June 1997). In the same part of the study area, however, nitrate concentration increased markedly in samples from well D24 following biosolids application in the area; it is not known whether nitrate concentration was increasing at this location prior to biosolids application. Chloride and cadmium concentrations in samples from well D24 remained small. Only one of the two bacteria samples from well D24 had detections of fecal streptococcus bacteria, and the count was less than the MRL. Phosphorus detections were small and erratic. Copper, nickel, and zinc generally were not detected in samples from well D24. The generally increasing concentrations of nitrate in samples from wells D9 and D24 may have resulted from biosolids applications in the area; however, the temporal patterns in the water-quality data for other constituent concentrations do not indicate much effect of biosolids applications on water quality during 1993-1998.

Biosolids were applied to the north part of the study area in the vicinity of well D25 (DC 304 and DC 305) in July 1994 and October 1997 through February 1998 (table 1; fig. 16). Biosolids were applied to areas upgradient from well D25 (DCs 303, 306, 307, and 308) in December 1993 through September 1997 (table 1; fig. 5). Nitrate concentrations spiked in samples from well D25 within 2.5 years after the initial biosolids application, decreased, and then spiked again within several months after the next biosolids application. Large chloride concentrations coincided with the largest nitrate concentrations in samples from well D25 (November 1997 and January 1998) (table 5). Cadmium was detected in all samples from well D25, but was reported at a slightly larger concentration in the December 1995 sample. Fecal streptococcus bacteria were reported above the MRL in an October 1996 sample from well D25. Phosphorus (and to a lesser extent organic nitrogen) concentrations were substantially larger in the November 1996 and June 1997 samples, the same samples that had larger concentrations of nitrate and cadmium. However, copper, nickel, and zinc were not detected above the MRL in samples from well D25. In general, the nitrate, chloride, and cadmium data from well D25 show a temporal pattern that could correspond with water-quality effects that lagged biosolids applications by about 1.5-2.5 years, although a longer period of data comparison would be needed to confirm this pattern.

In general, the comparison of temporal patterns of nitrate, chloride, cadmium, and fecal streptococcus bacteria with temporal patterns and magnitude (loading) of biosolids applications for well samples that contained at least some modern groundwater did not indicate biosolids effects on water quality. Only samples from well D25 had temporal concentration patterns in that seemed possibly related to biosolids applications.

\section{Other Indicators}

Levels of indicator bacteria can be used to evaluate sources of water-quality impairments (Gaggiani, 1991, p. 40; Murray and others, 2001). If the ratio of fecal coliform bacteria to fecal streptococcus bacteria in a sample is greater than 4.0, then humans likely are the source of the bacteria (Murray and others, 2001), although this ratio will not indicate the specific type 
of human fecal matter (such as septic, pit toilets, or biosolids) that contributed the bacteria. If the bacteria ratio is below 0.6 (Murray and others, 2001) or 0.7 (Gaggiani, 1991), then other warm-blooded animals such as cattle or wildlife likely are the source of the bacteria. Few samples from the study area had detections of both fecal coliform and fecal streptococcus bacteria (table 6); however, the sample data where ratios could be calculated indicated that humans were not the source of the bacteria in groundwater or surface-water samples.

The DG-CFC data can provide additional information about biosolids effects on water quality. As was mentioned previously, DG-CFC data can indicate apparent recharge dates for groundwater. Chlorofluorocarbon-113 (CFC-113) enrichment in groundwater samples can indicate that groundwater has been affected by some type of human wastewater; however, the chlorofluorocarbon data do not indicate which type of wastewater (biosolids, septic systems, historic outhouse effluent, or other sewage sources) affected the groundwater (E. Busenberg, U.S. Geological Survey, written commun., November 18, 1999). The chlorofluorocarbon data collected from the study area indicated that groundwater at wells D3, D9, and D24 may have been affected by wastewater sources. Historic homesteads are not known in the vicinity of wells D9 and D24, so biosolids are the most likely type of wastewater to have affected the groundwater at these locations. A homestead was present near well D3, so a pit toilet, septic system, or biosolids could have affected groundwater at this location. Even if CFC-113 enrichment indicates biosolids or some other wastewater type affected the groundwater, the presence of tracer constituents, such as DG-CFCs in water samples, does not constitute contamination or even adverse effects. Note that counts of fecal streptococcus bacteria were largest in samples from well D3 in November 1993 (8 months before biosolids were applied), but concentrations of other constituents such as nutrients and trace elements were not particularly large. In contrast, the large nitrate concentrations in samples from wells D9 and D24 in addition to CFC-113 enrichment indicate that biosolids could be the source of the nitrate in samples from wells D9 and D24. Thus, the presence of tracer constituents such as DG-CFCs along with elevated concentrations of other constituents (such as nitrate) can indicate sources of the elevated-concentration constituents.

In general, biosolids applications may have affected water quality slightly in parts of the study area during 1993-1998 through increases in nitrate and possibly a few other constituents, particularly where groundwater was oxic. However, increased nitrate concentrations were not widespread throughout the study area during the 1-5 years after biosolids applications began and were much less than those reported by Gaggiani (1991) for water-quality sites at a sewage-disposal area that was about $40 \mathrm{mi}$ farther west in Colorado. For most water-quality constituents, any effects from biosolids on water quality of the study area during 1993-1998 were obscured by high variation in concentrations, and the effects of biosolids applications on water quality of the study area during 1993-1999 were less than natural geological or microbiological effects.

\section{Summary}

The Metro Wastewater Reclamation District (Metro District) in Denver, Colo., applied biosolids resulting from municipal sewage treatment to farmland in eastern Colorado beginning in December 1993. The biosolids were a treated sewage product that met State and Federal regulatory criteria and were applied at agronomic loading rates as a fertilizer and soil amendment.

In mid-1993, the U.S. Geological Survey in cooperation with the Metro District began monitoring water quality at the biosolids-application area about 10 miles (mi) east of Deer Trail, Colo., to evaluate baseline water quality and the combined effects of natural processes, land uses, and biosolids applications on water quality of the biosolids-application area. The study included limited sampling of surface water and the unsaturated zone, but primarily focused on the sampling of groundwater, which was more abundant. The study area was on the eastern margin of the Denver Basin, a bowl-shaped sequence of sedimentary rocks that was formed in an ocean or near-ocean environment. The surficial geology of the study area consisted of interbedded shale, siltstone, and sandstone, with unconsolidated clay, silt, sand, and gravel. The primary water-supply aquifer in the study area was the Laramie-Fox Hills aquifer. Multiple alluvial aquifers associated with the surficial drainage network were present. Depth to groundwater below land surface at sampling locations ranged from a few feet to about 100 feet (ft). Most streams were ephemeral, so surface water predominantly was in the form of ponds. The climate was semi-arid with most of the precipitation occurring as rainfall in spring and in late summer. Land use was rangeland or cropland. The primary crop was winter wheat; farmland was not irrigated. Herbicides and other chemicals were applied to the study area for farming and weed-control purposes. Biosolids were applied as the only fertilizer and soil amendment on the Metro District properties during the study.

Baseline sampling was done in 1993 to provide information about water quality in the study area before biosolids applications began. Reconnaissance sampling was done during 1993 through 1995 to establish the spatial variability in water quality of the study area. Routine sampling was done during 1995 through 1998 to establish the temporal variation in water quality after biosolids applications began. The water-quality samples were analyzed by the Metro District laboratory for selected major ions, nutrients, and trace elements in filtered samples and bacteria (fecal coliform and fecal streptococcus bacteria) in unfiltered samples. Specialty sampling for unsaturated-zone pore water was initiated by the USGS in 1994 to determine if pore-water chemistry under biosolids-applied farmland differed from pore-water chemistry under farmland that was not applied with biosolids. Specialty sampling for dissolved gases and chlorofluorocarbons (CFCs) was done at selected monitoring wells in 1998 to infer information about water quality, flow paths, and groundwater recharge. Specialty sampling for oxidation-reduction (redox) information was done in 1999 to determine redox states in the groundwater. Other specialty monitoring included the use of automated data 
recorders during 1997 and 1998 to provide detailed information about selected water-quality characteristics. Corechemistry data from well borings in the study area also were obtained for comparison with the water-quality data.

Baseline water quality was marginal at some sites and better at other sites. Large concentrations of chloride, sulfate, nitrate, iron, and manganese were detected in some baseline samples. Sulfate concentrations in baseline samples were largest in samples from well D6. Nitrogen in baseline samples predominantly was in the form of nitrate. One-half of the sites sampled for baseline water quality had nitrate concentrations of 1 milligram per liter as nitrogen $(\mathrm{mg}-\mathrm{N} / \mathrm{L})$ or greater, five sites had baseline nitrate concentrations greater than $3 \mathrm{mg}-\mathrm{N} / \mathrm{L}$, three sites had baseline nitrate concentrations in the range of 5-10 mg-N/L, and one site (well D9) had baseline nitrate concentrations greater than $10 \mathrm{mg}-\mathrm{N} / \mathrm{L}$. Concentrations of iron and manganese were detected above the minimum reporting level (MRL) in baseline samples from various monitoring wells in the study area. Chloride was detected in nearly all baseline samples; some concentrations were large. Phosphorus (orthophosphate as phosphorus) and cadmium were detected at small concentrations in most baseline samples. Chromium and copper were not detected in any baseline samples, but nickel and zinc were detected in baseline samples. Bacteria (fecal streptococcus) were found in baseline samples from two of the 10 sampled wells. The baseline data indicate that major-ion, nutrient, traceelement, and bacteria sources other than biosolids were present in the study area and that water in the study area was of variable quality before biosolids ever were applied.

Variability in water quality of the study area was evident from the baseline data as well as from the data collected after biosolids applications began. The hydrology of the study area likely accounts for some of the variability in water quality. Precipitation caused runoff from farmed fields, caused intermittent and ephemeral streams to flow, and fed natural and anthropogenic ponds. During dry periods, pond water evaporated and desiccation cracks formed. Groundwater recharged preferentially from ponds and through desiccation cracks and coarse-grained deposits and outcrops, not uniformly through the unsaturated zone beneath all fields.

Concentrations of major ions, nutrients, trace elements, and bacteria varied spatially in the study area. Concentrations of sulfate, nitrate, iron, and manganese varied the most. Majorion concentrations in groundwater varied more by lithology or geologic formation than by drainage basin, biosolids treatment, site type, or well depth. Nitrate concentrations generally were largest in shallow bedrock-aquifer wells in the north part of the study area and smallest near wetland areas. Fecal coliform and fecal streptococcus bacteria were not detected in most of the samples from the study area, although fecal streptococcus bacteria were detected more frequently than fecal coliform bacteria, and pond samples generally had larger bacteria counts than nearby wells. Water-quality concentration did not correlate well with depth of sample, but smaller chloride, nitrate, and manganese concentrations were characteristic of deeper wells and the largest nitrate concentrations detected in study-area water was in pore-water samples from the $1.5-\mathrm{ft}$ depth. The pore-water data indicated that chloride, nitrate and copper reservoirs in the upper $3 \mathrm{ft}$ of the unsaturated zone could eventually migrate to the saturated zone.

Temporal variability in water quality generally was larger at some sites than other sites in the study area and larger for some water-quality constituents (like nitrate, iron, manganese, and fecal streptococcus bacteria) than other constituents. Nitrate concentrations in samples from wells D6, D24, and D32 increased more markedly and more consistently than in those from other wells in the study area. In general, nitrate concentration increased in samples from most wells along lower Cottonwood Creek but was more variable in samples from wells D9, D14, D19, D25, D28, and D31. Of the limited analytes considered for this study, nitrate increased in concentration the most consistently over time.

Groundwater samples from the study area exceeded various Colorado regulatory standards. Groundwater concentrations exceeded Colorado human-health or drinking-water standards for chloride in samples from well D6; for sulfate in samples from most wells; for nitrate in samples from wells D9, D24, D25, and D32; for nickel in sample from wells D4, D6, D12, D16, and D32; and for chromium, iron, and manganese in samples from many wells. Concentrations generally were less than Colorado agricultural standards, except for iron concentrations in samples from wells D21 and D29; nickel concentrations in samples from wells D6, D16, and D32; and manganese concentrations in samples from many wells. Baseline groundwater concentrations of chloride, sulfate, nitrate, iron, and manganese in samples from some wells exceeded Colorado regulatory standards before biosolids applications to the study area began.

Geology affected water quality in the study area. Major-ion and trace-element chemistry of water in the study area appears to be controlled primarily by local geology. Sandstone units in the study area contain iron-rich concretions and weather chemically to produce secondary crystalline minerals and amorphous iron oxides. Chemical analysis of core samples confirmed the presence of many of the same major and trace elements in the near-surface geologic materials of the study area that were in the groundwater. The dissolution and precipitation of common minerals such as gypsum, calcite, dolomite, halite, magnesiumsulfate, and pyrite minerals likely controlled the calcium, chloride, magnesium, sodium, and sulfate concentrations in the groundwater from many of the monitoring wells. Major-ion and trace-element chemistry of water in the study area therefore appears to have been controlled by local geology. In addition, characteristics of bedrock and soil such as cementation, porosity, and permeability likely determined groundwater flow paths and affected groundwater flow rates and chemistry.

Meteorology affected water quality in the study area through precipitation on surface water (wet deposition) that delivered atmospheric constituents to the water and through runoff and infiltration that transported constituents from the land surface into surface water or groundwater. Other sources of ions in the study area likely contributed a greater mass of constituents to the surface and subsurface water in the study area than the direct deposition of precipitation. The data 
indicate that meteorology could have affected water quality in the study area by mobilizing chemical constituents from the soil during runoff and infiltration. Warm surface temperatures coupled with low humidity and relatively small precipitation rates can result in evapotranspiration rates that exceeded precipitation-infiltration rates. Solutes can become concentrated in ponds, soil, and pore water. The pore-water data from the study area indicated that reservoirs of some solutes such as chloride, nitrate, and possibly copper were present in the soil near the surface and could be transported to the aquifers through a larger wetting front or through preferential flow paths. During drier periods, much of the recharge to groundwater likely happened at focused recharge areas in stream valleys, outcrop areas, or areas that had desiccation cracks. Geology appears to have affected water quality in the study area more than meteorology during 1993-1998.

Microbiological effects on water quality of the study area predominantly were the microbially mediated cycling of redox-sensitive constituents. Groundwater at most sites in the study area was oxic. Groundwater at some sites in the study area was depleted in oxygen (post oxic). Denitrification was a common process in groundwater of the study area, especially at wells D13 and D25. Chlorofluorocarbon data indicated that denitrification had occurred in groundwater at wells D6, D9, and D24, yet denitrification was not sufficient to prevent nitrate concentrations from increasing during 1993-1999 at these sites because the groundwater usually was oxic. Where denitrification is actively occurring, nitrate is reduced to nitrite and even to nitrogen gas; therefore, nitrate produced from additional nitrogen inputs to that site (such as from biosolids or other fertilizers) will be consumed in post-oxic groundwater. Oxygen reduction likely was the predominant terminal electron-acceptor process at sites where groundwater was oxic. Ferric-iron reduction (wells D15, D16, D21, and D23) or sulfate reduction (wells D22 and D30) was the predominant terminal electronacceptor process at sites where groundwater was post-oxic. In general, microbial processes in oxygen-depleted shallow groundwater (such as in much of the Muddy Creek area) have kept nitrate concentrations small and could reduce additional nitrogen inputs to the groundwater. Pond presence often resulted in microbial effects on water quality in the study area. Ponds provided a source of oxygenated recharge water, and associated wetlands created reducing zones.

Some flow-path effects were evident from the waterquality data collected during 1993-1999. Dissolved-gas and chlorofluorocarbon data indicated mixed old and young groundwater at wells D3, D6, D9, D13, D17, and D24 in 1998. Dissolved-gas and chlorofluorocarbon data indicated that groundwater followed a slow flow path after recharge before traveling to wells D3, D5, D6, D13, D14, D17, and D25, but at least some of the groundwater followed a rapid flow path after recharge to travel to wells D6, D9, and D24. Redox data indicated that a significant flow path for the Muddy Creek alluvial aquifer was from well D21 towards well D15, and that tributary alluvial aquifers in the vicinity of wells D20 and D17 likely flowed toward and joined the Muddy Creek alluvial aquifer, but do not indicate that the Laramie-Fox Hills aquifer
6 mi north of well D15 was part of this flow path in 1999. Preliminary geochemical modeling indicated that the groundwater flow path for well D6 likely did not include the bedrock-aquifer water (as represented by well D29) or groundwater from other upgradient shallow wells, but could include bedrock groundwater from a different location or simply rain water that interacted with rocks and either coal or organic matter (representing biosolids) in the vicinity of the well.

Biosolids effects on water quality were challenging to identify. The baseline data collected from the study area in 1993 were adequate to indicate that sources of major ions, nutrients, trace elements, and bacteria affected water quality before biosolids were applied. Chloride, sulfate, nitrate, and manganese concentrations in some baseline groundwater samples exceeded Colorado regulatory standards. Most chemicalconstituent concentrations were similar in the baseline samples as in later post-biosolids-application samples for 1994-1998. Because of the large spatial and temporal variability in water quality for both the baseline and the post-application data and the similarity in concentrations before and after biosolids application, effects from biosolids applications were not discernible from a simple comparison of baseline data with post-application data. Of the inorganic biosolids-signature constituents for aqueous media that were analyzed routinely in both the baseline and post-biosolids-application waterquality samples, only nitrate concentrations increased after biosolids applications began. The single control site available for this study area was of marginal use in evaluating biosolids effects on water quality for 1993-1999. The control site was not representative of all the other groundwater locations in the study area before biosolids applications began, particularly with respect to physical properties and concentrations of major ions, nitrogen, iron, and manganese. Differences in groundwater physical properties and concentrations of major ions, nitrogen, iron, and manganese between the control site and the biosolids-treatment area should not be attributed to biosolids but to geological and microbiological effects on water quality. Only samples from well D25 had temporal concentration patterns that seemed possibly related to biosolids applications. The sample data where ratios of fecal coliform bacteria to fecal streptococcus bacteria could be calculated indicated that humans were not the source of the bacteria in groundwater or surface-water samples. The chlorofluorocarbon data collected from the study area indicated that groundwater at wells D3, D9, and D24 may have been affected by wastewater sources. In general, biosolids applications may have affected water quality slightly in parts of the study area during 1993-1998 through increases in nitrate and possibly a few other constituents, particularly where groundwater was oxic. However, increased nitrate concentrations were not widespread throughout the study area during the $1-5$ years after biosolids applications began. For most water-quality constituents, any effects from biosolids on water quality of the study area during 1993-1998 were obscured by high variation in concentrations, and the effects of biosolids applications on water quality of the study area during 1993-1999 were less than natural geological or microbiological effects. 


\section{Acknowledgments}

The USGS thanks the land owners for allowing USGS instrument or well installations on their property. The USGS thanks the Metro District for providing water-quality analyses for this monitoring program. The author thanks James A. Tindall for design, installation, and sampling of the unsaturated-zone plots. The author thanks Peter B. McMahon for redox analysis and interpretation.

\section{References Cited}

Berti, W.R., and Jacobs, L.W., 1996, Chemistry and phytotoxicity of soil trace elements from repeated sewage sludge applications: Journal of Environmental Quality, v. 25, p. $1,025-1,032$.

Berti, W.R., and Jacobs, L.W., 1998, Distribution of trace elements in soil from repeated sewage sludge applications: Journal of Environmental Quality, v. 27, p. 1,280-1,286.

Busenberg, Eurybiades, and Plummer, L.N., 1992, Use of chlorofluorocarbons $\left(\mathrm{CCl}_{3} \mathrm{~F}\right.$ and $\left.\mathrm{CCl}_{2} \mathrm{~F}_{2}\right)$ as hydrologic tracers and age-dating tool: Example-The alluvium and terrace system of central Oklahoma: Water Resources Research, v. 28, p. 2,257-2,284.

Busenberg, Eurybiades, Plummer, L.N., Bartholomay, R.C., and Wayland, J.E., 1999, Chorofluorocarbons, sulfur hexafluoride, and dissolved permanent gases in groundwater from selected sites in and near the Idaho National Engineering and Environmental Laboratory, Idaho, 1994-1997: U.S. Geological Survey Open File Report 98-274, 72 p.

Chapelle, F.H., McMahon, P.B., Dubrovsky, N.M., Fujii, R.F., Oaksford, E.T., and Vroblesky, D.A., 1995, Deducing the distribution of terminal electron-accepting processes in hydrologically diverse groundwater systems: Water Resources Research, v. 31, no. 2, p. 359-371.

Colorado Department of Public Health and Environment, 1997, Basic standards for groundwater: 5CCR 1002-41, July 14, 1997, 56 p.

Colorado Department of Public Health and Environment, 1998, Biosolids regulation: 5CCR 1002-64, January 12, 1998, $53 \mathrm{p}$.

Drever, J.I., 1988, The geochemistry of natural waters (2d ed.): New Jersey, Prentice Hall, p. 328-329.

Drew, L.J., Schuenemeyer, J.H., and Bawiec, W.J., 1979, Petroleum exhaustion maps of the cretaceous "D-J" sandstone stratigraphic interval of the Denver Basin: U.S. Geological Survey Miscellaneous Investigations Series Map I-1138, scale 1:200,000, sheet 2.
Eljarrat, Ethel, Caixach, Josep, and Rivera, Josep, 1997, Effects of sewage sludges contaminated with polychlorinated dibenzo-p-dioxins, dibenzofurans, and biphenyls on agricultural soils: Environmental Science and Technology, v. 31 , no. 10 , p. $2,765-2,771$.

Farrar, J.W., and Long, Keith, 1996, Report on the U.S. Geological Survey's evaluation program for standard reference samples distributed in April 1996-T-139 (Trace constituents), T-141 (Trace constituents), M-138 (Major constituents), N-49 (Nutrient constituents), N-50 (Nutrient constituents), P-26 (Low-ionic strength constituents) and Hg-22 (Mercury): U.S. Geological Survey Open File Report 96-436, 143 p.

Freeze, R.A., and Cherry, J.A., 1979, Groundwater: New Jersey, Prentice-Hall Inc., 604 p.

Friedman, L.C., and Erdmann, D.E., 1982, Quality-assurance practices for the chemical and biological analyses of water and fluvial sediments: U.S. Geological Survey Techniques of Water-Resources Investigations, book 5, chap. A6, 181 p.

Gaggiani, N.G., 1991, Effects of land disposal of municipal sewage sludge on soil, streambed sediment, and groundand surface-water quality at a site near Denver, Colorado: U.S. Geological Survey Water-Resources Investigations Report 90-4106, 163 p.

Hem, J.D., 1992, Study and interpretation of the chemical characteristics of natural water (3d ed): U.S. Geological Survey Water-Supply Paper 2254, 263 p.

Horowitz, A.J., Demas, C.R., Fitzgerald, K.K., Miller, T.L., and Rickert, D.A., 1994, U.S. Geological Survey protocol for the collection and processing of surface-water samples for the subsequent determination of inorganic constituents in filtered water: U.S. Geological Survey Open-File Report 94-539, $57 \mathrm{p}$.

Johncox, D.A. and Gaggiani, N.G., 1991, Selected hydrologic data for farmland treated with sewage sludge near Platteville, Colorado, 1985-91: U.S. Geological Survey OpenFile Report 91-529, 120 p.

Koterba, M.T., Wilde, F.D., and Lapham, W.W., 1995, Groundwater data-collection protocols and procedures for the National Water-Quality Assessment programCollection and documentation of water-quality samples and related data: U.S. Geological Survey Open-File Report 95-399, 113 p.

Larsen, L.S., and Baber, T.G., Wesswick, E.L., McCoy, D.E., and Harman, J.B., 1966, Soil survey of Elbert County, Colorado: U.S. Department of Agriculture Soil Conservation Service Soil Survey series, $79 \mathrm{p}$.

Larsen, L.S., and Brown, J.B., 1971, Soil survey of Arapahoe County, Colorado: U.S. Department of Agriculture Soil Conservation Service Soil Survey series, 78 p. 
Lovley, D.K., Chapelle, F.H., and Woodward, J.C., 1994, Use of dissolved $\mathrm{H}_{2}$ concentrations to determine distribution of microbially catalyzed redox reactions in groundwater: Environmental Science and Technology, v. 28, no. 7, p. $1,205-1,210$.

Lull, K.J., and Gaggiani, N.G., 1996, Groundwater-quality data for the land application of sewage sludge at a site near Denver, Colorado, 1988-93: U.S. Geological Survey OpenFile Report 96-410, 164 p.

Lüscher, Martin, 1994, A portable high-quality random number generator for lattice field theory simulations: Computer Physics Communications, v. 79, no. 1, p. 100-110.

Major, T.J., Robson, S.G., Romero, J.C., and Zawistowski, Stanley, 1983, Hydrogeologic data from parts of the Denver Basin, Colorado: U.S. Geological Survey Open-File Report 83-274, 425 p.

McMahon, P.B., and Chapelle, F.H., 2008, Redox processes and water quality of selected principal aquifer systems: Ground Water, v. 46, p. 259-271.

Moolenaar, S.W., and Beltrami, P., 1998, Heavy metal balances of an Italian soil as affected by sewage sludge and Bordeaux mixture applications: Journal of Environmental Quality, v. 27, p. 828-835.

Murray, K.S., Fisher, L.E., Therrien, J., George, B., and Gillespie, J., 2001, Assessment and use of indicator bacteria to determine sources of pollution to an urban river: Journal of Great Lakes Research, v. 27, no. 2, p. 220-229.

Myers, D.N., and Sylvester, M.A., 1997, Fecal indicator bacteria, in Myers, D.N., and Wilde, F.D., eds., Biological indicators: U.S. Geological Survey Techniques of WaterResources Investigations, book 9, chap. A7, p. 1-16.

Nagamoto, C.T., Parungo, F., Reinking, R., Pueschel, R., and Gerish, T., 1983, Acid clouds and precipitation in eastern Colorado: Atmospheric Environment, v. 17, no. 5, p. 1,073-1,983.

Pais, Istvan, and Jones, J.B. Jr., 1997, The handbook of trace elements: Boca Raton, Florida, St. Lucie Press, 223 p.

Pirkey, K.D., and Glodt, S.R., 1998, Quality control at the U.S. Geological Survey National Water Quality Laboratory: U.S. Geological Survey Fact Sheet FS-026-98, 4 p.

Plummer, L.N., and Friedman, L.C., 1999, Tracing and dating young groundwater: U.S. Geological Survey Fact Sheet FS-134-99, 4 p.

Plummer, L.N., Prestemon, E.C., and Parkhurst, D.L., 1994, an interactive code (NETPATH) for modeling NET geochemical reactions along a flow PATH-version 2.0: U.S. Geological Survey Water-Resources Investigations Report 94-4169, $130 \mathrm{p}$.

Robson, S.G., 1977, Groundwater quality near a sewagesludge recycling site and a landfill near Denver, Colorado: U.S. Geological Survey Water-Resources Investigations Report 76-132, 137 p.
Robson, S.G., 1981, Geologic structure, hydrology, and water quality of the Laramie-Fox Hills aquifer in the Denver Basin, Colorado; U.S. Geological Survey Hydrologic Investigations Atlas HA-650, 3 sheets.

Robson, S.G., and Banta, E.R, 1995, Groundwater atlas of the United States, segment 2, Arizona, Colorado, New Mexico, Utah: U.S. Geological Survey Hydrologic Investigations Atlas 730-C, p. C20-C22.

Seaber, P.R., Kapinos, F.P., and Knapp, G.L., 1987, Hydrologic unit maps: U.S. Geological Survey Water-Supply Paper 2294, 63 p.

Sharps, J.A., 1980, Geologic map of the Limon $1 \times 2$ quadrangle, Colorado and Kansas: U.S. Geological Survey Miscellaneous Investigations Series Map I-1250, scale 1:250,000, sheet 1 .

Sloan, J.J., Dowdy, R.H., and Dolan, M.S., 1998, Recovery of biosolids-applied heavy metals sixteen years after application: Journal of Environmental Quality, v. 27, no. 6, p. $1,312-1,317$.

Soister, P.E., 1974, A preliminary report on a zone containing thick lignite beds, Denver Basin, Colorado: U.S. Geological Survey Open-File Report 74-27, 62 p.

Stanley, D.L., Boozer, T.M., and Schroder, L.J., 1998, Summary of the U.S. Geological Survey National Field Quality Assurance Program from 1979 through 1997: U.S. Geological Survey Open-File Report 98-392, 11 p.

Steenhuis, T.S., McBride, M.B., Richards, B.K., and Harrison, Ellen, 1999, Trace metal retention in the incorporation zone of land-applied sludge: Environmental Science and Technology, v. 33, no. 8, p. 1,171-1,174.

Stevens, M.R., Yager, T.J.B., Smith, D.B., and Crock, J.G., 2003, Biosolids, soils, ground-water, and streambedsediment data for a biosolids-application area near Deer Trail, Colorado, 1999: U.S. Geological Survey Open-File Report 02-51, 118 p.

Stevenson, F.J., 1994, Humus Chemistry: Genesis, Composition, Reactions: John Wiley and Sons, Inc, 496 p.

Stute, M., Schlosser, P., Clark, J.F., and Broecker, W.S., 1992, Paleotemperatures in the southwestern United States derived from noble gases in groundwater: Science, v. 256, p. 1,000-1,003.

Sylvester, M.A., Kister, L.R., and Garrett, W.B., eds., 1990, Guidelines for the collection, treatment, and analysis of water samples: U.S. Geological Survey Western Region Field Manual, $144 \mathrm{p}$.

Tindall, J.A., and Kunkel, J.R., 1999, Unsaturated zone hydrology for scientists and engineers: New Jersey, Prentice Hall, 624 p.

Tindall, J.A., Lull, K.J., and Gaggiani, N.G., 1994, Effects of land disposal of municipal sewage sludge on fate of nitrates in soil, streambed sediment, and water quality: Journal of Hydrology, v. 163, p. 147-185. 
Tindall, J.A. and Vencill, W.K., 1995, Transport of atrazine, 2,4-D, and dicamba through preferential flow path in an unsaturated claypan soil near Centralia, Missouri: Journal of Hydrology, v. 166, p. 37-59.

U.S. Environmental Protection Agency, 1993 (revised July 1, 2003), Part 503 - Standards for the use or disposal of sewage sludge: Code of Federal Regulations title 40, v. 27, 40CFR503, p. 820-952 available online at http://www.access.gpo.gov/nara/ cfr/waisidx_02/40cfr503_02.html

U.S. Geological Survey, 1976, Arapahoe County, Colorado: U.S. Geological Survey County Map Series (Topographic), scale 1:50,000, sheet 3 of 3 .

U.S. Geological Survey, 1980a, Elbert County, Colorado: U.S. Geological Survey County Map Series (Topographic), scale 1:50,000, sheet 2 of 3 .

U.S. Geological Survey, 1980b, Land use and land cover, 1975, Limon, Colorado; Kansas: U.S. Geological Survey Land Use Series L-191, scale 1:250,000, 1 sheet.

U.S. Geological Survey, variously dated, National field manual for the collection of water-quality data: U.S. Geological Survey Techniques of Water-Resources Investigations, book 9, chaps. A1-A9. (Also available at http://pubs.water.usgs.gov/twri9A.)
Wilson, G.B., and McNeill, G.W., 1997, Noble gas recharge temperatures and the excess air component: Applied Geochemistry, v. 12, p. 747-762.

Yager, T.J.B., and Arnold, L.R., 2003, Hydrogeology of a biosolids-application site near Deer Trail, Colorado, 1993-99: U.S. Geological Survey Water-Resources Investigations Report 03-4209, 90 p.

Yager, T.J.B., and McMahon, P.B., 2012, Preliminary assessment of sources of nitrogen in groundwater at a biosolidsapplication area near Deer Trail, Colorado, 2005: U.S. Geological Survey Scientific Investigations Report 2012-5056, 30 p. available online at http://pubs.usgs.gov/sir/2012/5056/.

Yager, T.J.B., Smith, D.B., and Crock, J.G., 2004a, Biosolids, soil, crop, ground-water, and streambed-sediment data for a biosolids-application area near Deer Trail, Colorado, 2002-2003: U.S. Geological Survey Open-File Report 2004-1404, 90 p.

Yager, T.J.B., Smith, D.B., and Crock, J.G., 2004b, Effects of surface applications of biosolids on soil, crops, groundwater, and streambed sediment near Deer Trail, Colorado, 1999-2003: U.S. Geological Survey Scientific Investigations Report 2004-5289, 93 p. 

Appendixes 
Appendix 1. Information for U.S. Geological Survey monitoring wells near Deer Trail, Colorado, 1993 through 1998.

[Lithologic descriptions and diagrams of well parts were reported by Yager and Arnold (2003); diagrams of well parts and other information for monitoring wells installed in 1999 as part of the U.S. Geological Survey Expanded Monitoring Program near Deer Trail were reported by Stevens and others (2003). Latitude and longitude are in the format degrees minutes seconds referenced to North American Datum of 1983; stickup, the length of well casing above ground (fluctuates because land surface is uneven and changes height over time), and measuring point is at the top of the stickup; bmp, below measuring point on the stickup; sump, the nonperforated closed casing below the screeen; U, not known; HUC, hydrologic unit code in the format 101900XX from Seaber and others (1987); altitude is in feet above North American Vertical Datum of 1988; km, kilometers; N, North; W, West; <, less than; Metro District, Metro District Wastewater Reclamation District; --, no data]

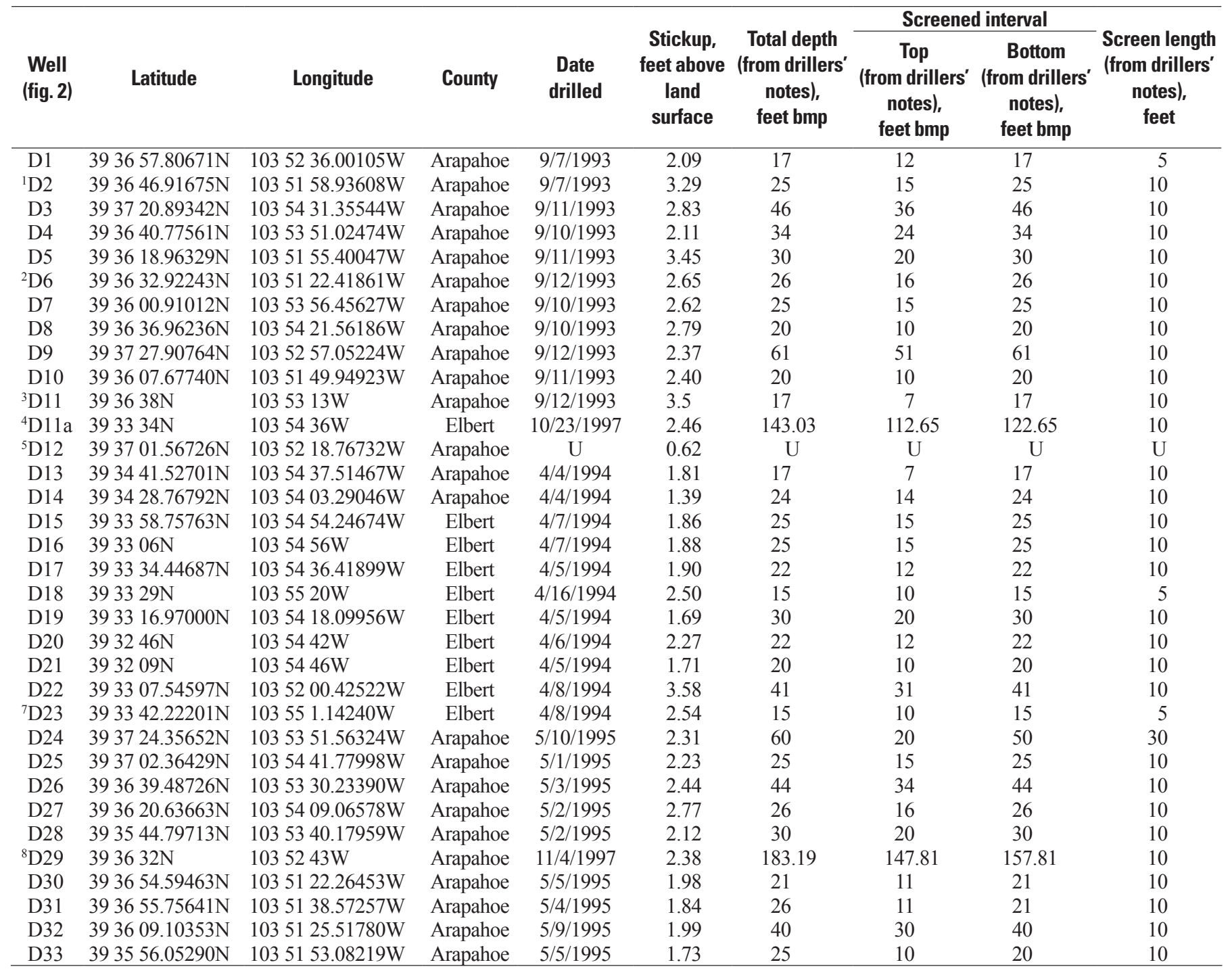


Appendix 1. Information for U.S. Geological Survey monitoring wells near Deer Trail, Colorado, 1993 through 1998.—Continued

[Lithologic descriptions and diagrams of well parts were reported by Yager and Arnold (2003); diagrams of well parts and other information for monitoring wells installed in 1999 as part of the U.S. Geological Survey Expanded Monitoring Program near Deer Trail were reported by Stevens and others (2003). Latitude and longitude are in the format degrees minutes seconds referenced to North American Datum of 1983; stickup, the length of well casing above ground (fluctuates because land surface is uneven and changes height over time), and measuring point is at the top of the stickup; bmp, below measuring point on the stickup; sump, the nonperforated closed casing below the screeen; U, not known; HUC, hydrologic unit code in the format 101900XX from Seaber and others (1987); altitude is in feet above North American Vertical Datum of 1988; km, kilometers; N, North; W, West; <, less than; Metro District, Metro District Wastewater Reclamation District; --, no data]

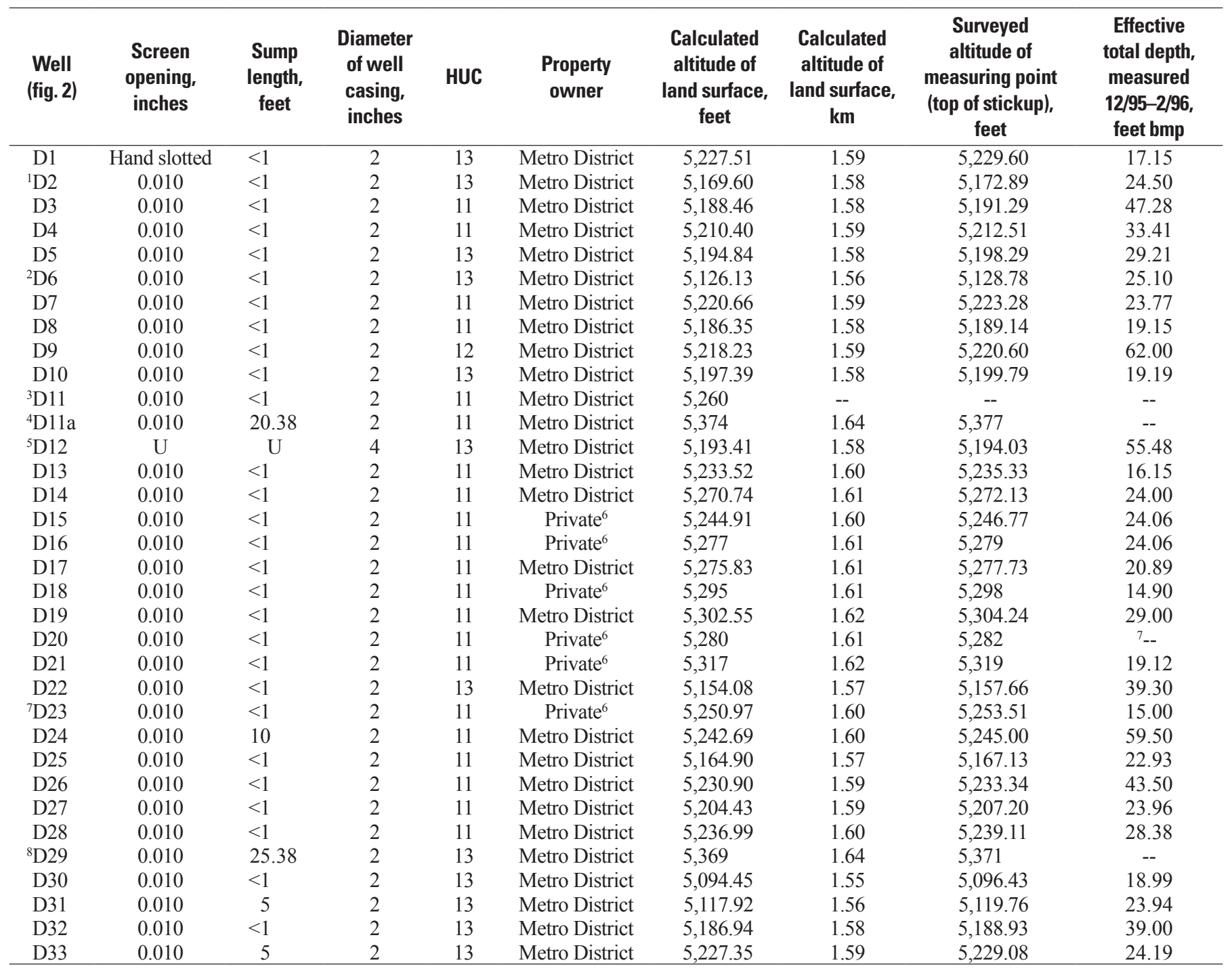

${ }^{1}$ Well had continuous-recorder instrumentation for water-level, water-temperature, and precipitation data from 1995 through 1998.

${ }^{2}$ Well had continuous-recorder instrumentation for specific conductance and water-temperature data from 1996 through 1998.

${ }^{3}$ Well was closed on 9/7/1995.

${ }^{4}$ Well information from geologist's notes.

${ }^{5}$ This well was not installed by the USGS. The well was installed by a previous land owner before 1993 and once contained a pump powered by a windmill for watering livestock.

${ }^{6}$ Property was owned by Metro Wastewater Reclamation District in 1993 and 1994, then transferred to a private land owner in 1995.

${ }^{7}$ Accuracy of depth measurement in question because well seemed to have an object immersed in the well from before October 1995.

${ }^{8}$ Well had continuous-recorder instrumentation for water-level, water-temperature, and precipitation data from 1994 through 1998. 
Appendix 2. Chemical symbols and abbreviations used in reporting chemical data in the appendixes.

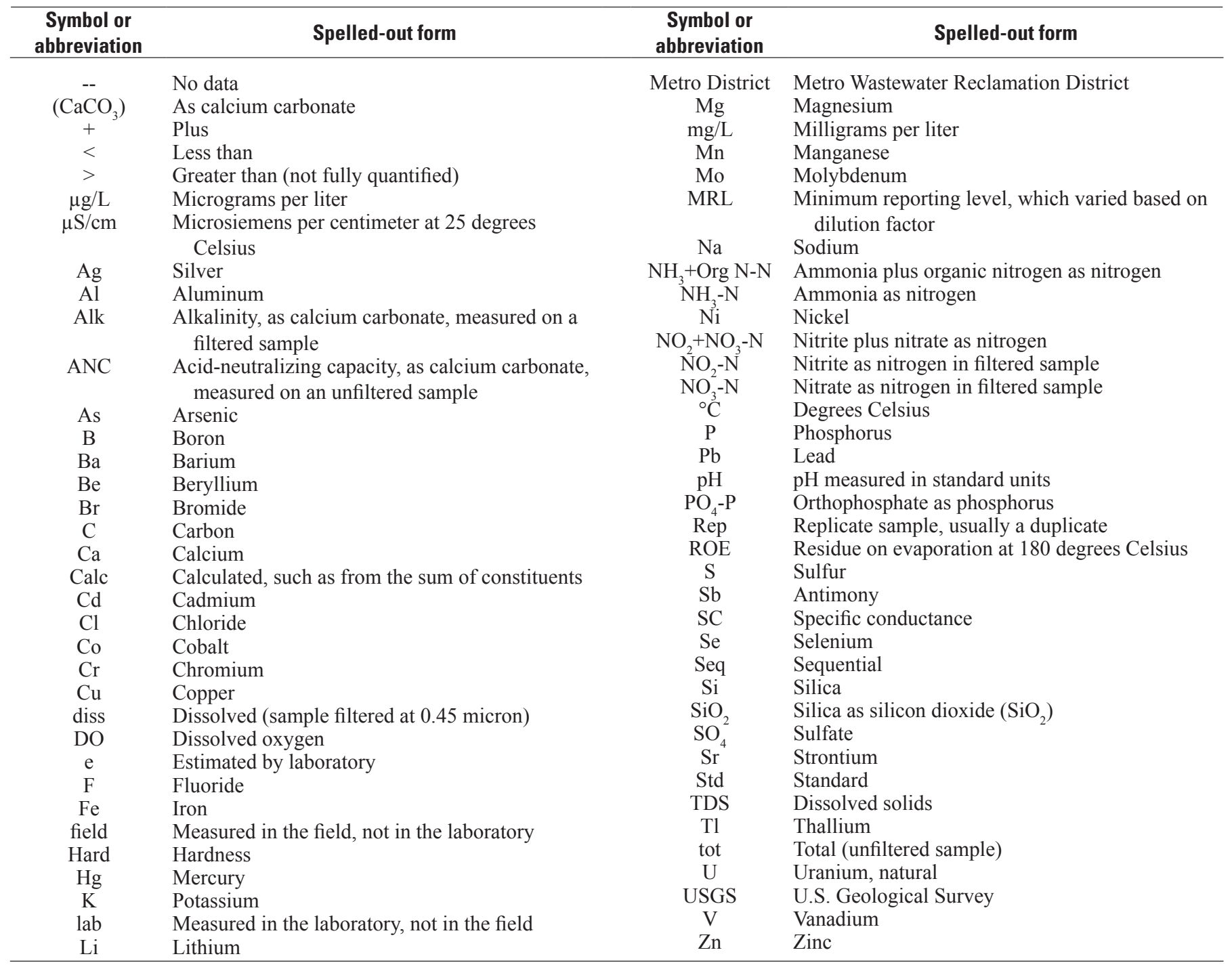


Appendix 3. Physical properties and major-ion data for replicate water-quality samples collected near Deer Trail, Colorado, 1993 through 1998.

[All abbreviations listed in appendix 2; data from filtered samples except ANC, pH, and SC data; $\mu \mathrm{S} / \mathrm{cm}$, microsiemens per centimeter at 25 degrees Celsius; $\mathrm{mg} / \mathrm{L}$, milligrams per liter; Rep, replicate; $\mu \mathrm{g} / \mathrm{L}$, micrograms per liter; Metro District, Metro Wastewater Reclamation District; --, no data; Seq, sequential; C1, first composite; C2, second composite; <, less than]

\begin{tabular}{|c|c|c|c|c|c|c|c|c|c|c|c|c|c|c|c|c|c|c|}
\hline $\begin{array}{l}\text { Sample } \\
\text { date }\end{array}$ & $\begin{array}{l}\text { Sample } \\
\text { site }\end{array}$ & $\begin{array}{l}\text { Sample } \\
\text { type }\end{array}$ & $\begin{array}{c}\text { Sample } \\
\text { sub-type }\end{array}$ & $\begin{array}{l}\text { Analyzing } \\
\text { laboratory }\end{array}$ & $\begin{array}{l}\text { ANC, } \\
\mathrm{lab}, \\
\mathrm{mg} / \mathrm{L}\end{array}$ & $\begin{array}{l}\text { Alk, } \\
\text { lab, } \\
\text { mg/L }\end{array}$ & $\begin{array}{c}\text { Hard } \\
\left(\mathrm{CaCO}_{3}\right), \\
\mathrm{mg} / \mathrm{L}\end{array}$ & $\begin{array}{l}\text { pH, } \\
\text { lab }\end{array}$ & $\begin{array}{c}\text { SC, } \\
\text { lab, } \\
\mu S / c m\end{array}$ & $\begin{array}{c}\text { TDS } \\
\text { (ROE), } \\
\text { mg/L }\end{array}$ & $\begin{array}{l}\mathrm{Ca}, \\
\mathrm{mg} / \mathrm{L}\end{array}$ & $\begin{array}{c}\mathrm{Cl}, \\
\mathrm{mg} / \mathrm{L}\end{array}$ & $\begin{array}{c}F, \\
m g / L\end{array}$ & $\begin{array}{c}\mathrm{K}, \\
\mathrm{mg} / \mathrm{L}\end{array}$ & $\begin{array}{l}\mathrm{Mg}, \\
\mathrm{mg} / \mathrm{L}\end{array}$ & $\begin{array}{l}\mathrm{Na}, \\
\mathrm{mg} / \mathrm{L}\end{array}$ & $\begin{array}{l}\mathrm{SiO}_{2^{\prime}} \\
\mathrm{mg} / \mathrm{L}\end{array}$ & $\begin{array}{l}\mathrm{SO}_{\mathrm{q}^{\prime}} \\
\mathrm{mg} / \mathrm{L}\end{array}$ \\
\hline 7/20/1994 & D22 & Rep & None & Metro District & -- & 573 & 5,120 & -- & -- & 11,400 & 371 & 55 & -- & 17.6 & 1,250 & 1,530 & -- & 7,680 \\
\hline $7 / 21 / 1994$ & D14 & Rep & None & Metro District & -- & 139 & 350 & -- & -- & 457 & 107 & 4 & -- & 1.9 & 19 & 31 & -- & 159 \\
\hline 9/30/1994 & D23 & Rep & None & Metro District & -- & 371 & 1,880 & -- & -- & 2,870 & 512 & 11 & -- & 4.4 & 104 & 184 & -- & 1,680 \\
\hline 10/3/1994 & D10 & Rep & Seq & Metro District & -- & 357 & 2,920 & -- & -- & -- & 518 & 28 & -- & 19.5 & 440 & 313 & -- & 3,330 \\
\hline $10 / 3 / 1994$ & D10 & Rep & Seq & USGS & -- & -- & 2,900 & 7.2 & 4,760 & 4,980 & 490 & 24 & 0.60 & -- & 400 & 270 & 24 & 3,100 \\
\hline $7 / 12 / 1995$ & D7 & Rep & None & Metro District & -- & 184 & 1,320 & -- & -- & 2,030 & 348 & 18 & -- & 3.0 & 116 & 60 & -- & 1,240 \\
\hline $7 / 12 / 1995$ & D25 & Rep & None & Metro District & -- & 298 & 2,160 & -- & -- & 3,460 & 581 & 95 & -- & 6.0 & 165 & 157 & -- & 2,050 \\
\hline 9/20/1995 & D31 & Rep & None & Metro District & -- & 533 & 5,500 & -- & -- & 9,430 & 396 & 100 & -- & 6.0 & 1,020 & 747 & -- & 5,700 \\
\hline 9/26/1995 & D20 & Rep & None & Metro District & -- & 261 & 1,920 & -- & -- & 2,808 & 428 & 7 & -- & 8.0 & 155 & 148 & -- & 1,720 \\
\hline $12 / 14 / 1995$ & D15 & Split & $\mathrm{C} 1$ & Metro District & -- & 527 & 2,810 & -- & -- & 4,860 & 502 & 19 & -- & 5.0 & 318 & 503 & -- & 2,990 \\
\hline $12 / 14 / 1995$ & D15 & Split & $\mathrm{C} 2$ & Metro District & -- & 526 & 2,540 & -- & -- & 4,890 & 425 & 19 & -- & 3.0 & 316 & 487 & -- & 2,760 \\
\hline $12 / 14 / 1995$ & D15 & Split & $\mathrm{C} 2$ & USGS & -- & -- & 2,400 & 6.9 & 4,980 & 5,060 & 470 & 17 & 2.0 & -- & 300 & 450 & 17 & 2,900 \\
\hline $12 / 14 / 1995$ & D3 & Split & $\mathrm{C} 1$ & Metro District & -- & 110 & 1,630 & -- & -- & 2,320 & 460 & 28 & -- & 4.0 & 104 & 50 & -- & 1,360 \\
\hline $12 / 14 / 1995$ & D3 & Split & $\mathrm{C} 2$ & Metro District & -- & 112 & 1,440 & -- & -- & 2,250 & 434 & 28 & -- & 4.0 & 106 & 54 & -- & 1,340 \\
\hline $12 / 14 / 1995$ & D3 & Split & $\mathrm{C} 2$ & USGS & -- & -- & 1,400 & 7.3 & 2,390 & 2,260 & 400 & 28 & 1.3 & -- & 100 & 62 & 30 & 1,400 \\
\hline $3 / 8 / 1996$ & D5 & Split & $\mathrm{C} 1$ & Metro District & -- & 246 & 2,480 & -- & -- & 4,210 & 512 & 39 & -- & 4.0 & 268 & 229 & -- & 2,800 \\
\hline $3 / 8 / 1996$ & D5 & Split & $\mathrm{C} 2$ & Metro District & -- & 218 & 2,500 & -- & -- & 4,220 & 518 & 40 & -- & 4.0 & 300 & 227 & -- & 2,720 \\
\hline $3 / 8 / 1996$ & D5 & Split & $\mathrm{C} 2$ & USGS & -- & -- & 2,400 & 7.3 & 4,150 & 4,090 & 480 & 30 & 0.50 & -- & 300 & 240 & 20 & 2,600 \\
\hline $6 / 14 / 1996$ & D9 & Split & $\mathrm{C} 1$ & Metro District & -- & 252 & 1,500 & -- & -- & 2,190 & 418 & 14 & -- & $<0.1$ & 102 & 37 & -- & 1,260 \\
\hline $6 / 14 / 1996$ & D9 & Split & $\mathrm{C} 2$ & Metro District & -- & 254 & 1,460 & -- & -- & 2,220 & 420 & 14 & -- & $<0.1$ & 102 & 37 & -- & 1,240 \\
\hline $6 / 14 / 1996$ & D9 & Split & $\mathrm{C} 2$ & USGS & -- & -- & 1,600 & 7.3 & 2,340 & 2,240 & 450 & 9.1 & 1.1 & -- & 110 & 45 & 25 & 1,200 \\
\hline $8 / 22 / 1996$ & D17 & Split & $\mathrm{C} 2$ & Metro District & -- & 236 & 264 & -- & -- & 292 & 57 & 3 & -- & 2.0 & 25 & 10 & -- & 40 \\
\hline $8 / 22 / 1996$ & D17 & Split & $\mathrm{C} 2$ & USGS & 235 & -- & 230 & 7.7 & 515 & 295 & 55 & 2.0 & 1.8 & -- & 22 & 15 & 20 & 39 \\
\hline $8 / 22 / 1996$ & D17 & Split & $\mathrm{C} 1$ & Metro District & -- & 232 & 278 & -- & -- & 302 & 57 & 3 & -- & 2.0 & 26 & 10 & -- & 38 \\
\hline 11/8/1996 & D25 & Split & $\mathrm{C} 1$ & Metro District & -- & 598 & 3,880 & -- & -- & 5,660 & 836 & 151 & -- & 7.0 & 340 & 336 & -- & 2,640 \\
\hline $11 / 8 / 1996$ & D25 & Split & $\mathrm{C} 2$ & Metro District & -- & 591 & 4,000 & -- & -- & 5,680 & 884 & 151 & -- & 7.0 & 348 & 330 & -- & 2,960 \\
\hline $11 / 8 / 1996$ & D25 & Split & $\mathrm{C} 2$ & USGS & 596 & -- & 3,300 & 7.2 & 5,480 & 5,580 & 740 & 160 & 1.3 & -- & 340 & 350 & 35 & 3,000 \\
\hline 2/26/1997 & D15 & Split & $\mathrm{C} 1$ & Metro District & -- & 541 & 2,580 & -- & -- & 4,990 & 514 & 20 & -- & 4.0 & 359 & 590 & -- & 3,340 \\
\hline 2/26/1997 & D15 & Split & $\mathrm{C} 2$ & Metro District & -- & 547 & 2,680 & -- & -- & 5,050 & 474 & $<1$ & -- & $<0.1$ & 334 & 550 & -- & 3,150 \\
\hline 2/26/1997 & D15 & Split & $\mathrm{C} 2$ & USGS & 551 & -- & 2,400 & 6.9 & 5,010 & 4,790 & 450 & 46 & 1.8 & -- & 310 & 500 & 15 & 3,100 \\
\hline $2 / 26 / 1997$ & D23 & Rep & None & Metro District & -- & 365 & 1,870 & -- & -- & 2,890 & 538 & 9 & -- & 6.0 & 116 & 185 & -- & 1,950 \\
\hline 6/3/1997 & D2 & Rep & None & Metro District & -- & 270 & 3,420 & -- & -- & 4,740 & 482 & 17 & -- & $<0.1$ & 415 & 206 & -- & 2,880 \\
\hline 6/5/1997 & D10 & Split & $\mathrm{C} 1$ & USGS & 387 & -- & 2,700 & 7.4 & 4,570 & 4,630 & 440 & 19 & 0.6 & -- & 380 & 270 & 21 & 2,900 \\
\hline 6/5/1997 & D10 & Split & $\mathrm{C} 2$ & Metro District & -- & 404 & 4,210 & -- & -- & 4,790 & 448 & 20 & -- & 15.0 & 393 & 294 & -- & 2,680 \\
\hline 6/5/1997 & D10 & Split & $\mathrm{C} 2$ & Metro District & -- & 392 & 3,510 & -- & -- & 4,760 & 447 & 20 & -- & 14.0 & 388 & 288 & -- & 3,060 \\
\hline
\end{tabular}


Appendix 3. Physical properties and major-ion data for replicate water-quality samples collected near Deer Trail, Colorado, 1993 through 1998.-Continued

[All abbreviations listed in appendix 2; data from filtered samples except ANC, pH, and SC data; $\mu \mathrm{S} / \mathrm{cm}$, microsiemens per centimeter at 25 degrees Celsius; $\mathrm{mg} / \mathrm{L}$, milligrams per liter; Rep, replicate; $\mu \mathrm{g} / \mathrm{L}$, micrograms per liter; Metro District, Metro Wastewater Reclamation District; --, no data; Seq, sequential; C1, first composite; C2, second composite; <, less than]

\begin{tabular}{|c|c|c|c|c|c|c|c|c|c|c|c|c|c|c|c|c|c|c|}
\hline $\begin{array}{c}\text { Sample } \\
\text { date }\end{array}$ & $\begin{array}{l}\text { Sample } \\
\text { site }\end{array}$ & $\begin{array}{c}\text { Sample } \\
\text { type }\end{array}$ & $\begin{array}{c}\text { Sample } \\
\text { sub-type }\end{array}$ & $\begin{array}{l}\text { Analyzing } \\
\text { laboratory }\end{array}$ & $\begin{array}{l}\text { ANC, } \\
\text { lab, } \\
\text { mg/L }\end{array}$ & $\begin{array}{l}\text { Alk, } \\
\text { lab, } \\
\text { mg/L }\end{array}$ & $\begin{array}{c}\text { Hard } \\
\left(\mathrm{CaCO}_{3}\right), \\
\mathrm{mg} / \mathrm{L}\end{array}$ & $\begin{array}{l}\mathrm{pH}, \\
\text { lab }\end{array}$ & $\begin{array}{c}\text { SC, } \\
\text { lab, } \\
\mu S / c m\end{array}$ & $\begin{array}{c}\text { TDS } \\
\text { (ROE), } \\
\text { mg/L }\end{array}$ & $\begin{array}{c}\mathrm{Ca}, \\
\mathrm{mg} / \mathrm{L}\end{array}$ & $\begin{array}{c}\mathrm{Cl}, \\
\mathrm{mg} / \mathrm{L}\end{array}$ & $\begin{array}{c}\mathrm{F}, \\
\mathrm{mg} / \mathrm{L}\end{array}$ & $\begin{array}{c}\mathrm{K} \\
\mathrm{mg} / \mathrm{L}\end{array}$ & $\begin{array}{l}\mathrm{Mg}, \\
\mathrm{mg} / \mathrm{L}\end{array}$ & $\begin{array}{c}\mathrm{Na}, \\
\mathrm{mg} / \mathrm{L}\end{array}$ & $\begin{array}{l}\mathrm{SiO}_{2^{\prime}} \\
\mathrm{mg} / \mathrm{L}\end{array}$ & $\begin{array}{l}\mathrm{SO}_{4^{\prime}} \\
\mathrm{mg} / \mathrm{L}\end{array}$ \\
\hline $8 / 27 / 1997$ & D25 & Split & $\mathrm{C} 2$ & Metro District & -- & 310 & 2,290 & -- & -- & 3,810 & 612 & 111 & -- & 3.0 & 159 & 168 & -- & 2,210 \\
\hline 8/27/1997 & D25 & Split & $\mathrm{C} 2$ & USGS & 310 & -- & 2,200 & 7.3 & 3,720 & 3,850 & 610 & 100 & 1 & -- & 160 & 180 & 25 & 2,100 \\
\hline 8/27/1997 & D25 & Split & $\mathrm{C} 1$ & Metro District & -- & 321 & 2,300 & -- & -- & 3,820 & 610 & 110 & -- & $<0.1$ & 164 & 187 & -- & 1,810 \\
\hline $8 / 27 / 1997$ & D31 & Rep & None & Metro District & -- & 559 & 4,960 & -- & -- & 8,780 & 424 & 87 & -- & $<0.1$ & 887 & 768 & -- & 6,030 \\
\hline 11/17/1997 & D9 & Rep & None & Metro District & -- & 227 & 1,660 & -- & -- & 2,900 & 464 & 12 & -- & 4.0 & 143 & 58 & -- & 1,590 \\
\hline 11/19/1997 & D25 & Split & $\mathrm{C} 1$ & Metro District & -- & 493 & 2,480 & -- & -- & 4,620 & 707 & 126 & -- & 11.0 & 245 & 294 & -- & 2,500 \\
\hline $11 / 19 / 1997$ & D25 & Split & $\mathrm{C} 2$ & Metro District & -- & 505 & 2,540 & -- & -- & 4,640 & 798 & 127 & -- & 11.0 & 260 & 318 & -- & 2,730 \\
\hline $11 / 19 / 1997$ & D25 & Split & $\mathrm{C} 2$ & USGS & 502 & -- & 2,700 & 7.3 & 4,590 & 4,710 & 700 & 120 & 1.1 & -- & 230 & 280 & 28 & 2,500 \\
\hline $1 / 6 / 1998$ & D9 & Rep & None & Metro District & -- & 207 & 1,450 & -- & -- & 2,830 & 500 & 7 & -- & 3.0 & 157 & 71 & -- & 1,720 \\
\hline $1 / 8 / 1998$ & D25 & Split & $\mathrm{C} 1$ & Metro District & -- & 478 & 2,760 & -- & -- & 4,550 & 690 & 116 & -- & $<0.1$ & 248 & 280 & -- & 2,490 \\
\hline $1 / 8 / 1998$ & D25 & Split & $\mathrm{C} 2$ & Metro District & -- & 474 & 2,730 & -- & -- & 4,590 & 699 & 119 & -- & $<0.1$ & 259 & 296 & -- & 2,530 \\
\hline $1 / 8 / 1998$ & D25 & Split & $\mathrm{C} 2$ & USGS & 510 & -- & 2,600 & 7.3 & 4,540 & 4,520 & 660 & 120 & 1.2 & -- & 220 & 270 & 29 & 2,500 \\
\hline $4 / 15 / 1998$ & D9 & Rep & None & Metro District & -- & 226 & 1,800 & -- & -- & 2,890 & 539 & 7 & -- & $<0.1$ & 143 & 55 & -- & 1,740 \\
\hline 4/16/1998 & D25 & Split & $\mathrm{C} 1$ & Metro District & -- & 471 & 2,700 & -- & -- & 4,510 & 820 & 120 & -- & 3.0 & 262 & 300 & -- & 2,810 \\
\hline $4 / 16 / 1998$ & D25 & Split & $\mathrm{C} 2$ & Metro District & -- & 472 & 2,900 & -- & -- & 4,570 & 752 & 119 & -- & $<0.1$ & 246 & 288 & -- & 2,500 \\
\hline $4 / 16 / 1998$ & D25 & Split & $\mathrm{C} 2$ & USGS & 494 & -- & 2,500 & 7.3 & 4,550 & 4,530 & 630 & 110 & 1 & -- & 230 & 280 & 27 & 2,400 \\
\hline $7 / 15 / 1998$ & D30 & Rep & None & Metro District & -- & 359 & 2,770 & -- & -- & 4,960 & 487 & 51 & -- & 4.0 & 457 & 372 & -- & 3,030 \\
\hline $7 / 16 / 1998$ & D25 & Split & $\mathrm{C} 1$ & Metro District & -- & 366 & 1,980 & -- & -- & 4,160 & 697 & 109 & -- & $<0.1$ & 179 & 209 & -- & 2,310 \\
\hline $7 / 16 / 1998$ & D25 & Split & $\mathrm{C} 2$ & Metro District & -- & 390 & 2,280 & -- & -- & 4,250 & 719 & 109 & -- & $<0.1$ & 194 & 227 & -- & 2,320 \\
\hline 7/16/1998 & D25 & Split & $\mathrm{C} 2$ & USGS & 368 & -- & 2,200 & 7.5 & 3,940 & 4,010 & 560 & 110 & 1 & -- & 180 & 210 & 25 & 2,200 \\
\hline
\end{tabular}


Appendix 4. Nutrient data for replicate water-quality samples collected near Deer Trail, Colorado, 1993 through 1998.

[All data from filtered samples unless otherwise noted; mg/L, milligrams per liter; Rep, replicate; Metro District, Metro Wastewater Reclamation District; --, no data; <, less than; Seq, sequential; C1, first composite; C2, second composite; FCA, filtered chilled acidified sample; WCA, whole-water chilled acidified sample; FCC, filtered chilled unacidified sample; other abbreviations listed in appendix 2]

\begin{tabular}{|c|c|c|c|c|c|c|c|c|c|c|c|c|}
\hline $\begin{array}{l}\text { Sample } \\
\text { date }\end{array}$ & $\begin{array}{l}\text { Sample } \\
\text { site }\end{array}$ & $\begin{array}{c}\text { Sample } \\
\text { type }\end{array}$ & $\begin{array}{l}\text { Sample } \\
\text { subtype }\end{array}$ & $\begin{array}{l}\text { Analyzing } \\
\text { laboratory }\end{array}$ & $\begin{array}{c}\mathrm{NH}_{3}+\text { Org N-N, } \\
\text { diss, mg/L }\end{array}$ & $\begin{array}{c}\mathrm{NH}_{3}+\mathrm{Org} \mathrm{N}-\mathrm{N}, \\
\text { tot, } \mathrm{mg} / \mathrm{L}\end{array}$ & $\begin{array}{c}\mathrm{NH}_{3}-\mathrm{N}, \\
\mathrm{mg} / \mathrm{L}\end{array}$ & $\begin{array}{c}\mathrm{NO}_{2}-\mathrm{N}, \\
\mathrm{mg} / \mathrm{L}\end{array}$ & $\begin{array}{c}\mathrm{NO}_{2}+\mathrm{NO}_{3}-\mathrm{N}, \\
\mathrm{mg} / \mathrm{L}\end{array}$ & $\begin{array}{c}\text { P, diss, } \\
\mathrm{mg} / \mathrm{L}\end{array}$ & $\begin{array}{l}\text { P, tot, } \\
\mathrm{mg} / \mathrm{L}\end{array}$ & $\begin{array}{c}\mathrm{PO}_{4}-\mathrm{P}, \\
\mathrm{mg} / \mathrm{L}\end{array}$ \\
\hline 7/20/1994 & D22 & Rep & None & Metro District & 1.2 & -- & $<0.1$ & 0.03 & 0.71 & -- & -- & $<0.02$ \\
\hline 7/21/1994 & D14 & Rep & None & Metro District & 0.5 & -- & $<0.1$ & 0.04 & 3.78 & -- & -- & $<0.02$ \\
\hline 9/30/1994 & D23 & Rep & None & Metro District & 1.6 & -- & 0.9 & $<0.02$ & $<0.02$ & -- & -- & 0.04 \\
\hline 10/3/1994 & D10 & Rep & Seq & Metro District & 0.3 & -- & $<0.1$ & $<0.02$ & 0.69 & -- & -- & 0.17 \\
\hline 10/3/1994 & D10 & Rep & Seq & USGS & 0.20 & -- & 0.020 & $<0.010$ & 0.65 & 0.13 & -- & 0.15 \\
\hline 7/12/1995 & D7 & Rep & None & Metro District & $<0.3$ & -- & 0.1 & $<0.02$ & 1.43 & -- & -- & 0.07 \\
\hline 7/12/1995 & D25 & Rep & None & Metro District & 0.8 & -- & 0.1 & $<0.02$ & 2.47 & -- & -- & 0.10 \\
\hline 9/20/1995 & D31 & Rep & None & Metro District & 0.3 & -- & $<0.1$ & 0.03 & 1.52 & -- & -- & 0.04 \\
\hline 9/26/1995 & D20 & Rep & None & Metro District & $<0.3$ & -- & $<0.1$ & $<0.02$ & $<0.02$ & -- & -- & 0.21 \\
\hline $12 / 14 / 1995$ & D15 & Split & $\mathrm{C} 1$ & Metro District & 1.9 & -- & 0.6 & $<0.02$ & $<0.02$ & -- & -- & 0.05 \\
\hline $12 / 14 / 1995$ & D15 & Split & $\mathrm{C} 2$ & Metro District & 1.4 & -- & 0.6 & $<0.02$ & $<0.02$ & -- & -- & $<0.02$ \\
\hline 12/14/1995 & D15 & Split & $\mathrm{C} 2$ & USGS & 1.4 & -- & 0.960 & $<0.010$ & $<0.05$ & 0.02 & -- & 0.02 \\
\hline 12/14/1995 & D3 & Split & $\mathrm{C} 1$ & Metro District & $<0.3$ & -- & $<0.1$ & $<0.02$ & 2.76 & -- & -- & 0.04 \\
\hline $12 / 14 / 1995$ & D3 & Split & $\mathrm{C} 2$ & Metro District & $<0.3$ & -- & $<0.1$ & $<0.02$ & 3.67 & -- & -- & $<0.02$ \\
\hline $12 / 14 / 1995$ & D3 & Split & $\mathrm{C} 2$ & USGS & $<0.20$ & -- & 0.080 & $<0.010$ & 3.70 & 0.04 & -- & 0.02 \\
\hline 3/8/1996 & D5 & Split & $\mathrm{C} 1$ & Metro District & $<0.3$ & -- & $<0.1$ & $<0.02$ & 6.22 & -- & -- & 0.03 \\
\hline 3/8/1996 & D5 & Split & $\mathrm{C} 2$ & Metro District & 0.6 & -- & $<0.1$ & $<0.02$ & 6.24 & -- & -- & $<0.02$ \\
\hline 3/8/1996 & D5 & Split & $\mathrm{C} 2$ & USGS & $<0.20$ & -- & 0.120 & $<0.010$ & 6.00 & $<0.01$ & -- & 0.02 \\
\hline 6/14/1996 & D9 & Split & $\mathrm{C} 1$ & Metro District & 0.5 & -- & $<0.1$ & $<0.02$ & 12.9 & -- & -- & 0.06 \\
\hline 6/14/1996 & D9 & Split & $\mathrm{C} 2$ & Metro District & 0.5 & -- & $<0.1$ & 0.02 & 12.8 & -- & -- & 0.06 \\
\hline $6 / 14 / 1996$ & D9 & Split & $\mathrm{C} 2$ & USGS & 0.40 & -- & 0.060 & $<0.010$ & 14.0 & 0.08 & -- & 0.06 \\
\hline $8 / 22 / 1996$ & D17 & Split & $\mathrm{C} 2$ & Metro District & 0.5 & -- & $<0.1$ & $<0.02$ & 0.50 & -- & -- & 0.08 \\
\hline $8 / 22 / 1996$ & D17 & Split & $\mathrm{C} 2$ & USGS & $<0.20$ & -- & $<0.015$ & 0.010 & 0.53 & 0.06 & -- & 0.09 \\
\hline $8 / 22 / 1996$ & D17 & Split & $\mathrm{C} 1$ & Metro District & 0.4 & -- & $<0.1$ & $<0.02$ & 0.48 & -- & -- & 0.08 \\
\hline 11/8/1996 & D25 & Split & $\mathrm{C} 1$ & Metro District & 1.4 & -- & $<0.1$ & 0.02 & 30.9 & -- & -- & 0.21 \\
\hline 11/8/1996 & D25 & Split & $\mathrm{C} 2$ & Metro District & 1.4 & -- & $<0.1$ & 0.02 & 29.9 & -- & -- & 0.19 \\
\hline 11/8/1996 & D25 & Split & $\mathrm{C} 2$ & USGS & 1.7 & -- & 0.300 & 0.040 & 34.0 & 0.19 & -- & 0.02 \\
\hline
\end{tabular}


Appendix 4. Nutrient data for replicate water-quality samples collected near Deer Trail, Colorado, 1993 through 1998.—Continued

[All data from filtered samples unless otherwise noted; mg/L, milligrams per liter; Rep, replicate; Metro District, Metro Wastewater Reclamation District; --, no data; <, less than; Seq, sequential; C1, first composite; C2, second composite; FCA, filtered chilled acidified sample; WCA, whole-water chilled acidified sample; FCC, filtered chilled unacidified sample; other abbreviations listed in appendix 2]

\begin{tabular}{|c|c|c|c|c|c|c|c|c|c|c|c|c|}
\hline $\begin{array}{c}\text { Sample } \\
\text { date }\end{array}$ & $\begin{array}{l}\text { Sample } \\
\text { site }\end{array}$ & $\begin{array}{c}\text { Sample } \\
\text { type }\end{array}$ & $\begin{array}{l}\text { Sample } \\
\text { subtype }\end{array}$ & $\begin{array}{l}\text { Analyzing } \\
\text { laboratory }\end{array}$ & $\begin{array}{c}\mathrm{NH}_{3}+\mathrm{Org} \mathrm{N}-\mathrm{N}, \\
\text { diss, } \mathrm{mg} / \mathrm{L}\end{array}$ & $\begin{array}{c}\mathrm{NH}_{3}+\mathrm{Org} \mathrm{N}-\mathrm{N}, \\
\text { tot, } \mathrm{mg} / \mathrm{L}\end{array}$ & $\begin{array}{c}\mathrm{NH}_{3}-\mathrm{N}, \\
\mathrm{mg} / \mathrm{L}\end{array}$ & $\begin{array}{c}\mathrm{NO}_{2}-\mathrm{N}, \\
\mathrm{mg} / \mathrm{L}\end{array}$ & $\begin{array}{c}\mathrm{NO}_{2}+\mathrm{NO}_{3}-\mathrm{N}, \\
\mathrm{mg} / \mathrm{L}\end{array}$ & $\begin{array}{l}\text { P, diss, } \\
\mathrm{mg} / \mathrm{L}\end{array}$ & $\begin{array}{l}\text { P, tot, } \\
\mathrm{mg} / \mathrm{L}\end{array}$ & $\begin{array}{c}\mathrm{PO}_{4}-\mathrm{P}, \\
\mathrm{mg} / \mathrm{L}\end{array}$ \\
\hline $2 / 26 / 1997$ & D15 & Split & $\mathrm{C} 1$ & Metro District & 1.2 & -- & 0.4 & 0.02 & $<0.02$ & -- & -- & 0.05 \\
\hline $2 / 26 / 1997$ & D15 & Split & $\mathrm{C} 2$ & Metro District & 1.2 & -- & $<0.1$ & $<0.02$ & $<0.02$ & -- & -- & 0.03 \\
\hline 2/26/1997 & D15 & Split & $\mathrm{C} 2$ & USGS & 1.3 & -- & 0.890 & $<0.010$ & $<0.05$ & $<0.010$ & -- & 0.010 \\
\hline $2 / 26 / 1997$ & $\mathrm{D} 23$ & Rep & None & Metro District & 1.1 & -- & 0.7 & 0.02 & $<0.02$ & -- & -- & 0.05 \\
\hline $6 / 3 / 1997$ & $\mathrm{D} 2$ & Rep & None & Metro District & $<0.3$ & -- & $<0.1$ & $<0.02$ & 0.30 & -- & -- & 0.04 \\
\hline $6 / 5 / 1997$ & D10 & Split & $\mathrm{C} 1$ & USGS & $<0.20$ & -- & 0.015 & $<0.010$ & 3.60 & 0.14 & -- & 0.16 \\
\hline $6 / 5 / 1997$ & D10 & Split & $\mathrm{C} 2$ & Metro District & $<0.3$ & -- & $<0.1$ & $<0.02$ & 3.67 & -- & -- & 0.17 \\
\hline $6 / 5 / 1997$ & D10 & Split & $\mathrm{C} 2$ & Metro District & 0.4 & -- & $<0.1$ & $<0.02$ & 3.68 & -- & -- & 0.16 \\
\hline $8 / 27 / 1997$ & D25 & Split & $\mathrm{C} 2$ & Metro District & 0.6 & -- & $<0.1$ & $<0.02$ & 0.40 & -- & -- & 0.08 \\
\hline $8 / 27 / 1997$ & D25 & Split & $\mathrm{C} 2$ & USGS & 0.42 & -- & 0.110 & 0.014 & 0.31 & 0.081 & -- & 0.099 \\
\hline $8 / 27 / 1997$ & $\mathrm{D} 25$ & Split & $\mathrm{C} 1$ & Metro District & 0.6 & -- & $<0.1$ & $<0.02$ & 0.28 & -- & -- & 0.07 \\
\hline $8 / 27 / 1997$ & D31 & Rep & None & Metro District & 0.8 & -- & $<0.1$ & $<0.02$ & 0.08 & -- & -- & $<0.02$ \\
\hline $11 / 17 / 1997$ & D9 & Rep & None & Metro District & $<0.3$ & -- & $<0.1$ & $<0.02$ & 20.0 & -- & -- & 0.08 \\
\hline $11 / 19 / 1997$ & D25 & Split & $\mathrm{C} 1$ & Metro District & 1.2 & -- & $<0.1$ & $<0.02$ & 11.8 & -- & -- & 0.12 \\
\hline $11 / 19 / 1997$ & $\mathrm{D} 25$ & Split & $\mathrm{C} 2$ & Metro District & 1.2 & -- & $<0.1$ & $<0.02$ & 10.5 & -- & -- & 0.12 \\
\hline $11 / 19 / 1997$ & $\mathrm{D} 25$ & Split & $\mathrm{C} 2$ & USGS & 1.1 & -- & 0.100 & 0.015 & 13.0 & 0.12 & -- & 0.17 \\
\hline $1 / 6 / 1998$ & D9 & Rep & None & Metro District & $<0.3$ & -- & $<0.1$ & $<0.02$ & 21.4 & -- & -- & 0.05 \\
\hline $1 / 8 / 1998$ & D25 & Split & $\mathrm{C} 1$ & Metro District & 1.1 & -- & $<0.1$ & $<0.02$ & 12.1 & -- & -- & 0.12 \\
\hline $1 / 8 / 1998$ & D25 & Split & $\mathrm{C} 2$ & Metro District & 1.0 & -- & $<0.1$ & $<0.02$ & 12.5 & -- & -- & 0.12 \\
\hline $1 / 8 / 1998$ & D25 & Split & $\mathrm{C} 2$ & USGS & 1.0 & -- & 0.064 & 0.011 & 13.0 & 0.096 & -- & 0.15 \\
\hline $4 / 15 / 1998$ & D9 & Rep & None & Metro District & 0.5 & -- & $<0.1$ & $<0.02$ & 24.2 & -- & -- & $<0.02$ \\
\hline $4 / 16 / 1998$ & $\mathrm{D} 25$ & Split & $\mathrm{C} 1$ & Metro District & 0.9 & -- & $<0.1$ & $<0.02$ & 12.1 & -- & -- & 0.11 \\
\hline $4 / 16 / 1998$ & D25 & Split & $\mathrm{C} 2$ & Metro District & 1.1 & -- & $<0.1$ & $<0.02$ & 12.3 & -- & -- & 0.11 \\
\hline $4 / 16 / 1998$ & $\mathrm{D} 25$ & Split & $\mathrm{C} 2 \mathrm{FCA}, \mathrm{WCA}$ & USGS & 1.3 & 1.2 & 0.160 & 0.013 & 13.0 & 0.16 & 0.15 & 0.16 \\
\hline $4 / 16 / 1998$ & $\mathrm{D} 25$ & Split & $\mathrm{C} 2 \mathrm{FCC}$ & USGS & 1.1 & -- & 0.065 & -- & 13.0 & 0.15 & -- & -- \\
\hline $7 / 15 / 1998$ & D30 & Rep & None & Metro District & 0.7 & -- & $<0.1$ & $<0.02$ & $<0.02$ & -- & -- & 0.04 \\
\hline $7 / 16 / 1998$ & D25 & Split & $\mathrm{C} 1$ & Metro District & 1.0 & -- & $<0.1$ & $<0.02$ & 3.42 & -- & -- & 0.12 \\
\hline $7 / 16 / 1998$ & $\mathrm{D} 25$ & Split & $\mathrm{C} 2$ & Metro District & 1.0 & -- & $<0.1$ & $<0.02$ & 3.80 & -- & -- & 0.09 \\
\hline $7 / 16 / 1998$ & D25 & Split & C2 FCA, WCA & USGS & 0.62 & 0.60 & 0.066 & 0.016 & 3.70 & 0.13 & 0.12 & 0.11 \\
\hline $7 / 16 / 1998$ & $\mathrm{D} 25$ & Split & $\mathrm{C} 2 \mathrm{FCC}$ & USGS & 0.60 & -- & 0.068 & -- & 3.70 & 0.081 & -- & - \\
\hline
\end{tabular}


Appendix 5. Trace-element data for replicate water-quality samples collected near Deer Trail, Colorado, 1993 through 1998.

[All data from filtered samples; $\mu \mathrm{g} / \mathrm{L}$, micrograms per liter; Rep, replicate; Metro District, Metro Wastewater Reclamation District; --, no data; <, less than; Seq, sequential; C1, first composite; C2, second composite; other abbreviations listed in appendix 2]

\begin{tabular}{|c|c|c|c|c|c|c|c|c|c|c|c|c|c|c|c|c|c|c|c|}
\hline $\begin{array}{c}\text { Sample } \\
\text { date }\end{array}$ & $\begin{array}{c}\text { Sample } \\
\text { site }\end{array}$ & $\begin{array}{c}\text { Sample } \\
\text { type }\end{array}$ & $\begin{array}{c}\text { Sample } \\
\text { sub-type }\end{array}$ & $\begin{array}{l}\text { Analyzing } \\
\text { laboratory }\end{array}$ & $\begin{array}{l}\mathrm{Ag}, \\
\mu \mathrm{g} / \mathrm{L}\end{array}$ & $\begin{array}{l}\mathrm{Ba}, \\
\mu \mathrm{g} / \mathrm{L}\end{array}$ & $\begin{array}{l}\mathrm{Be}, \\
\mu \mathrm{g} / \mathrm{L}\end{array}$ & $\begin{array}{l}\text { Cd, } \\
\mu g / L\end{array}$ & $\begin{array}{c}\mathrm{Cr}, \\
\mu \mathrm{g} / \mathrm{L}\end{array}$ & $\begin{array}{l}\text { Co, } \\
\mu \mathrm{g} / \mathrm{L}\end{array}$ & $\begin{array}{c}\mathrm{Cu}, \\
\mu \mathrm{g} / \mathrm{L}\end{array}$ & $\begin{array}{l}\text { Fe, } \\
\mu \mathrm{g} / \mathrm{L}\end{array}$ & $\begin{array}{c}\mathrm{Li}, \\
\mu \mathrm{g} / \mathrm{L}\end{array}$ & $\begin{array}{l}\mathrm{Mn}, \\
\mu \mathrm{g} / \mathrm{L}\end{array}$ & $\begin{array}{l}\text { Mo, } \\
\mu \mathrm{g} / \mathrm{L}\end{array}$ & $\begin{array}{c}\mathrm{Ni}, \\
\mu \mathrm{g} / \mathrm{L}\end{array}$ & $\begin{array}{l}\mathrm{Pb}, \\
\mu \mathrm{g} / \mathrm{L}\end{array}$ & $\begin{array}{c}\begin{array}{c}V \\
\mu \mathrm{g} / \mathrm{L}\end{array} \\
\end{array}$ & $\begin{array}{c}\mathrm{Zn}, \\
\mu \mathrm{g} / \mathrm{L}\end{array}$ \\
\hline $7 / 20 / 1994$ & D22 & Rep & None & Metro District & -- & -- & -- & $<0.2$ & 180 & -- & $<40$ & $<100$ & -- & 1,220 & -- & $<40$ & $<10$ & -- & $<40$ \\
\hline 7/21/1994 & D14 & Rep & None & Metro District & -- & -- & -- & $<0.2$ & 20 & -- & $<100$ & $<250$ & -- & 250 & -- & $<100$ & $<10$ & -- & $<100$ \\
\hline 9/30/1994 & D23 & Rep & None & Metro District & -- & -- & -- & $<0.2$ & $<20$ & -- & $<20$ & 990 & -- & 16,050 & -- & $<20$ & $<10$ & -- & $<20$ \\
\hline $10 / 3 / 1994$ & D10 & Rep & Seq & Metro District & -- & -- & -- & 0.1 & $<100$ & -- & $<100$ & $<250$ & -- & $<100$ & -- & $<100$ & $<10$ & -- & $<100$ \\
\hline $10 / 3 / 1994$ & D10 & Rep & Seq & USGS & $<4$ & 14 & $<2$ & $<4$ & $<20$ & $<12$ & $<40$ & $<12$ & 220 & $<4$ & $<40$ & $<40$ & $<40$ & $<24$ & $<12$ \\
\hline $7 / 12 / 1995$ & D7 & Rep & None & Metro District & -- & -- & -- & 0.1 & $<20$ & -- & $<20$ & $<50$ & -- & 550 & -- & $<20$ & $<10$ & -- & $<20$ \\
\hline $7 / 12 / 1995$ & $\mathrm{D} 25$ & Rep & None & Metro District & -- & -- & -- & 0.1 & $<20$ & -- & $<20$ & $<50$ & -- & 2,300 & -- & $<20$ & $<10$ & -- & $<20$ \\
\hline $9 / 20 / 1995$ & D31 & Rep & None & Metro District & -- & -- & -- & $<0.2$ & $<100$ & -- & $<100$ & $<250$ & -- & 420 & -- & $<100$ & $<10$ & -- & $<100$ \\
\hline $9 / 26 / 1995$ & $\mathrm{D} 20$ & Rep & None & Metro District & -- & -- & -- & 0.1 & $<20$ & -- & $<20$ & $<50$ & -- & 250 & -- & $<20$ & $<10$ & -- & $<20$ \\
\hline $12 / 14 / 1995$ & D15 & Split & $\mathrm{C} 1$ & Metro District & -- & -- & -- & 0.1 & $<20$ & -- & $<20$ & 1,840 & -- & 7,810 & -- & 20 & $<10$ & -- & $<20$ \\
\hline $12 / 14 / 1995$ & D15 & Split & $\mathrm{C} 2$ & Metro District & -- & -- & -- & $<0.2$ & $<20$ & -- & $<20$ & 1,810 & -- & 7,940 & -- & 30 & $<10$ & -- & $<20$ \\
\hline $12 / 14 / 1995$ & D15 & Split & $\mathrm{C} 2$ & USGS & $<3$ & 13 & $<2$ & 6.0 & $<20$ & $<9$ & $<30$ & 2,000 & 140 & 7,400 & 30 & 30 & $<30$ & $<18$ & 16 \\
\hline $12 / 14 / 1995$ & D3 & Split & $\mathrm{C} 1$ & Metro District & -- & -- & - & 0.1 & $<20$ & -- & $<20$ & $<50$ & -- & 550 & -- & 40 & $<10$ & -- & $<20$ \\
\hline $12 / 14 / 1995$ & D3 & Split & $\mathrm{C} 2$ & Metro District & -- & -- & -- & 0.1 & $<20$ & -- & $<20$ & $<50$ & -- & 280 & -- & 20 & $<10$ & -- & $<20$ \\
\hline $12 / 14 / 1995$ & D3 & Split & $\mathrm{C} 2$ & USGS & 3 & 11 & $<2$ & 6.0 & $<15$ & $<9$ & $<30$ & $<9$ & 150 & 300 & 40 & 40 & $<30$ & $<18$ & $<9$ \\
\hline 3/8/1996 & D5 & Split & $\mathrm{C} 1$ & Metro District & -- & -- & -- & $<0.2$ & $<40$ & -- & $<40$ & $<100$ & -- & $<40$ & -- & $<40$ & $<10$ & -- & $<40$ \\
\hline $3 / 8 / 1996$ & D5 & Split & $\mathrm{C} 2$ & Metro District & -- & -- & -- & $<0.2$ & $<40$ & -- & $<40$ & $<100$ & -- & $<40$ & -- & $<40$ & $<10$ & -- & $<40$ \\
\hline $3 / 8 / 1996$ & D5 & Split & $\mathrm{C} 2$ & USGS & $<3$ & 9 & $<2$ & $<3.0$ & $<20$ & $<9$ & $<30$ & 26 & 160 & 3 & $<30$ & $<30$ & 110 & $<18$ & $<9$ \\
\hline $6 / 14 / 1996$ & D9 & Split & $\mathrm{C} 1$ & Metro District & -- & -- & -- & 0.1 & $<20$ & -- & $<20$ & $<50$ & -- & $<20$ & -- & $<20$ & $<10$ & -- & $<20$ \\
\hline $6 / 14 / 1996$ & D9 & Split & $\mathrm{C} 2$ & Metro District & -- & -- & -- & $<0.2$ & $<20$ & -- & $<20$ & 230 & -- & $<20$ & -- & $<20$ & $<10$ & -- & $<20$ \\
\hline $6 / 14 / 1996$ & D9 & Split & $\mathrm{C} 2$ & USGS & $<3$ & 14 & $<2$ & 4.0 & $<20$ & $<9$ & $<30$ & $<120$ & 85 & $<6$ & $<30$ & $<30$ & 50 & $<18$ & $<9$ \\
\hline $8 / 22 / 1996$ & D17 & Split & $\mathrm{C} 2$ & Metro District & -- & -- & -- & $<0.2$ & $<20$ & -- & $<20$ & $<50$ & -- & 220 & -- & $<20$ & $<10$ & -- & $<20$ \\
\hline $8 / 22 / 1996$ & D17 & Split & $\mathrm{C} 2$ & USGS & 2 & 57 & $<0.5$ & $<1.0$ & $<5$ & $<3$ & $<10$ & $<3$ & 29 & 210 & $<10$ & 10 & 20 & $<6$ & 12 \\
\hline $8 / 22 / 1996$ & D17 & Split & $\mathrm{C} 1$ & Metro District & -- & -- & -- & $<0.2$ & $<20$ & -- & $<20$ & $<50$ & -- & 200 & -- & $<20$ & $<10$ & -- & $<20$ \\
\hline $11 / 8 / 1996$ & D25 & Split & $\mathrm{C} 1$ & Metro District & -- & -- & -- & 0.2 & $<20$ & -- & $<20$ & $<50$ & -- & 1,340 & -- & $<20$ & $<10$ & -- & $<20$ \\
\hline $11 / 8 / 1996$ & D25 & Split & $\mathrm{C} 2$ & Metro District & -- & -- & -- & 0.3 & $<20$ & -- & $<20$ & $<50$ & -- & 1,520 & -- & $<20$ & $<10$ & -- & $<20$ \\
\hline $11 / 8 / 1996$ & D25 & Split & $\mathrm{C} 2$ & USGS & $<4$ & 21 & $<2$ & $<4$ & $<20$ & $<10$ & $<40$ & $<12$ & 120 & 1,600 & $<40$ & $<40$ & $<40$ & $<24$ & 23 \\
\hline
\end{tabular}


Appendix 5. Trace-element data for replicate water-quality samples collected near Deer Trail, Colorado, 1993 through 1998.-Continued

[All data from filtered samples; $\mu \mathrm{g} / \mathrm{L}$, micrograms per liter; Rep, replicate; Metro District, Metro Wastewater Reclamation District; --, no data; <, less than; Seq, sequential; C1, first composite; C2, second composite; other abbreviations listed in appendix 2]

\begin{tabular}{|c|c|c|c|c|c|c|c|c|c|c|c|c|c|c|c|c|c|c|c|}
\hline $\begin{array}{c}\text { Sample } \\
\text { date }\end{array}$ & $\begin{array}{c}\text { Sample } \\
\text { site }\end{array}$ & $\begin{array}{c}\text { Sample } \\
\text { type }\end{array}$ & $\begin{array}{c}\text { Sample } \\
\text { sub-type }\end{array}$ & $\begin{array}{l}\text { Analyzing } \\
\text { laboratory }\end{array}$ & $\begin{array}{l}\mathrm{Ag}, \\
\mu \mathrm{g} / \mathrm{L}\end{array}$ & $\begin{array}{l}\mathrm{Ba}, \\
\mu \mathrm{g} / \mathrm{L}\end{array}$ & $\begin{array}{c}\mathrm{Be}, \\
\mu \mathrm{g} / \mathrm{L}\end{array}$ & $\begin{array}{l}\text { Cd, } \\
\mu g / L\end{array}$ & $\begin{array}{c}\mathrm{Cr}, \\
\mu \mathrm{g} / \mathrm{L}\end{array}$ & $\begin{array}{l}\text { Co, } \\
\mu \mathrm{g} / \mathrm{L}\end{array}$ & $\begin{array}{c}\mathrm{Cu}, \\
\mu \mathrm{g} / \mathrm{L}\end{array}$ & $\begin{array}{c}\text { Fe, } \\
\mu \mathrm{g} / \mathrm{L}\end{array}$ & $\begin{array}{c}\mathrm{Li}, \\
\mu \mathrm{g} / \mathrm{L}\end{array}$ & $\begin{array}{l}\text { Mn, } \\
\mu g / L\end{array}$ & $\begin{array}{l}\text { Mo, } \\
\mu \mathrm{g} / \mathrm{L}\end{array}$ & $\begin{array}{c}\mathrm{Ni}, \\
\mu \mathrm{g} / \mathrm{L}\end{array}$ & $\begin{array}{l}\mathrm{Pb}, \\
\mu \mathrm{g} / \mathrm{L}\end{array}$ & $\begin{array}{c}V, \\
\mu g / L\end{array}$ & $\begin{array}{c}\mathrm{Zn}, \\
\mu \mathrm{g} / \mathrm{L}\end{array}$ \\
\hline $2 / 26 / 1997$ & $\mathrm{D} 15$ & Split & $\mathrm{C} 1$ & Metro District & -- & -- & -- & 0.1 & $<20$ & -- & $<20$ & 1,440 & -- & 7,700 & -- & $<20$ & $<10$ & -- & $<20$ \\
\hline $2 / 26 / 1997$ & D15 & Split & $\mathrm{C} 2$ & Metro District & -- & -- & -- & 0.1 & $<20$ & -- & $<20$ & 1,340 & -- & 7,880 & -- & $<20$ & $<10$ & -- & $<20$ \\
\hline $2 / 26 / 1997$ & D15 & Split & $\mathrm{C} 2$ & USGS & $<3$ & 13 & $<2$ & $<3$ & $<20$ & 10 & $<30$ & 1,400 & 140 & 7,200 & $<30$ & $<30$ & $<30$ & $<18$ & $<9$ \\
\hline $2 / 26 / 1997$ & D23 & Rep & None & Metro District & -- & -- & -- & $<0.2$ & $<20$ & -- & $<20$ & 1,640 & -- & 15,700 & -- & $<20$ & $<10$ & -- & $<20$ \\
\hline 6/3/1997 & D2 & Rep & None & Metro District & -- & -- & -- & $<0.2$ & 90 & -- & $<60$ & $<150$ & -- & $<60$ & -- & 30 & $<10$ & -- & 50 \\
\hline 6/5/1997 & D10 & Split & $\mathrm{C} 1$ & USGS & $<4$ & 9 & $<2$ & $<4$ & $<20$ & $<12$ & $<40$ & $<12$ & 180 & $<4$ & $<40$ & $<40$ & $<40$ & $<24$ & $<12$ \\
\hline 6/5/1997 & D10 & Split & $\mathrm{C} 2$ & Metro District & -- & -- & -- & 0.1 & 110 & -- & 50 & $<150$ & -- & $<60$ & -- & $<60$ & $<10$ & -- & 50 \\
\hline 6/5/1997 & D10 & Split & $\mathrm{C} 2$ & Metro District & -- & -- & -- & 0.1 & 110 & -- & 70 & $<150$ & -- & $<60$ & -- & 100 & $<10$ & -- & 20 \\
\hline $8 / 27 / 1997$ & D25 & Split & $\mathrm{C} 2$ & Metro District & -- & -- & -- & 0.2 & 60 & -- & $<40$ & $<100$ & -- & 2,380 & -- & $<40$ & $<10$ & -- & $<40$ \\
\hline $8 / 27 / 1997$ & D25 & Split & $\mathrm{C} 2$ & USGS & $<3$ & 15 & $<1.5$ & $<3$ & $<15$ & $<9$ & $<30$ & $<9$ & 73 & 2,300 & $<30$ & $<30$ & 50 & $<18$ & $<9$ \\
\hline $8 / 27 / 1997$ & D25 & Split & $\mathrm{C} 1$ & Metro District & -- & -- & -- & 0.2 & 40 & -- & $<40$ & $<100$ & -- & 2,380 & -- & $<40$ & $<10$ & -- & $<40$ \\
\hline $8 / 27 / 1997$ & D31 & Rep & None & Metro District & -- & -- & -- & $<0.2$ & 340 & -- & $<100$ & $<250$ & -- & 200 & -- & $<100$ & $<10$ & -- & $<100$ \\
\hline $11 / 17 / 1997$ & D9 & Rep & None & Metro District & -- & -- & -- & $<0.2$ & $<20$ & -- & $<20$ & $<50$ & -- & $<20$ & -- & 20 & $<10$ & -- & $<20$ \\
\hline 11/19/1997 & D25 & Split & C1 & Metro District & -- & -- & -- & 0.1 & $<20$ & -- & $<20$ & $<50$ & -- & 2,840 & -- & $<20$ & $<10$ & -- & $<20$ \\
\hline $11 / 19 / 1997$ & D25 & Split & $\mathrm{C} 2$ & Metro District & -- & -- & -- & 0.1 & $<20$ & -- & $<20$ & $<50$ & -- & 2,900 & -- & $<20$ & $<10$ & -- & $<20$ \\
\hline 11/19/1997 & D25 & Split & $\mathrm{C} 2$ & USGS & $<4$ & 21 & $<2$ & $<4$ & $<20$ & $<12$ & $<40$ & $<12$ & 100 & 2,700 & $<40$ & $<40$ & $<40$ & $<24$ & $<12$ \\
\hline 1/6/1998 & D9 & Rep & None & Metro District & -- & -- & -- & $<0.2$ & $<20$ & -- & $<20$ & $<50$ & -- & $<20$ & -- & $<20$ & $<10$ & -- & $<20$ \\
\hline $1 / 8 / 1998$ & D25 & Split & $\mathrm{C} 1$ & Metro District & -- & -- & -- & 0.2 & $<20$ & -- & $<20$ & $<50$ & -- & 2,290 & -- & $<20$ & $<10$ & -- & $<20$ \\
\hline $1 / 8 / 1998$ & D25 & Split & $\mathrm{C} 2$ & Metro District & -- & -- & -- & 0.3 & $<20$ & -- & $<20$ & $<50$ & -- & 2,330 & -- & $<20$ & $<10$ & -- & $<20$ \\
\hline $1 / 8 / 1998$ & D25 & Split & $\mathrm{C} 2$ & USGS & $<12$ & 18 & $<3$ & $<24$ & $<42$ & $<36$ & $<30$ & $<30$ & 99 & 2,100 & $<180$ & $<120$ & $<300$ & $<30$ & $<60$ \\
\hline $4 / 15 / 1998$ & D9 & Rep & None & Metro District & -- & -- & -- & 0.1 & $<40$ & -- & $<40$ & $<100$ & -- & $<40$ & -- & $<40$ & $<10$ & -- & $<40$ \\
\hline $4 / 16 / 1998$ & D25 & Split & $\mathrm{C} 1$ & Metro District & -- & -- & -- & 0.2 & $<40$ & -- & $<40$ & $<100$ & -- & 2,190 & -- & $<40$ & $<10$ & -- & $<40$ \\
\hline $4 / 16 / 1998$ & $\mathrm{D} 25$ & Split & $\mathrm{C} 2$ & Metro District & -- & -- & -- & 0.2 & $<40$ & -- & $<40$ & $<100$ & -- & 2,240 & -- & $<40$ & $<10$ & -- & $<40$ \\
\hline 4/16/1998 & D25 & Split & $\mathrm{C} 2$ & USGS & $<12$ & 17 & $<3$ & $<24$ & $<42$ & $<36$ & $<30$ & $<30$ & 99 & 2,300 & -- & $<120$ & $<300$ & $<30$ & -- \\
\hline $7 / 15 / 1998$ & D30 & Rep & None & Metro District & -- & -- & -- & $<0.2$ & 80 & -- & $<40$ & 290 & -- & 250 & -- & $<40$ & $<10$ & -- & $<40$ \\
\hline 7/16/1998 & D25 & Split & $\mathrm{C} 1$ & Metro District & -- & -- & -- & 0.2 & 60 & -- & $<40$ & $<100$ & -- & 2,520 & -- & $<40$ & $<10$ & -- & $<40$ \\
\hline 7/16/1998 & D25 & Split & $\mathrm{C} 2$ & Metro District & -- & -- & -- & 0.2 & 80 & -- & $<40$ & $<100$ & -- & 2,300 & -- & $<40$ & $<10$ & -- & $<40$ \\
\hline $7 / 16 / 1998$ & D25 & Split & $\mathrm{C} 2$ & USGS & $<12$ & 16 & $<3$ & $<24$ & $<42$ & $<36$ & $<30$ & $<30$ & 86 & 2,300 & $<180$ & $<120$ & $<300$ & $<30$ & $<60$ \\
\hline
\end{tabular}


Appendix 6. Codes used in reporting information and chemical data (appendix 7) for blank water-quality samples collected near Deer Trail, Colorado, 1993 through 1999.

[USGS, U.S. Geological Survey]

\begin{tabular}{|c|c|}
\hline \multicolumn{2}{|r|}{ Sampling type } \\
\hline 2 & Groundwater inorganic-sampling blank \\
\hline 3 & Pore water (unsaturated zone) inorganic-sampling blank \\
\hline \multicolumn{2}{|r|}{ Type of blank } \\
\hline FB & Field blank \\
\hline EB & Equipment blank \\
\hline \multicolumn{2}{|r|}{ Sub-type } \\
\hline A & Acids only \\
\hline $\mathrm{F}$ & All field plus lab conditions, including equipment \\
\hline E & All equipment or specific equipment \\
\hline \multicolumn{2}{|r|}{ 2. } \\
\hline $\mathrm{CD}$ & USGS laboratory in Denver, Colorado \\
\hline $\mathrm{L}$ & Lowry former sewage-sludge disposal site near Denver, Colorado \\
\hline $\mathrm{D \#}$ & Deer Trail project well site \\
\hline \multicolumn{2}{|r|}{ Ambient condition } \\
\hline $\mathrm{D}$ & Dusty \\
\hline $\mathrm{R}$ & Road dust and exhaust \\
\hline $\mathrm{B}$ & Biosolids recently applied \\
\hline $\mathrm{P}$ & Pesticides recently applied \\
\hline $\mathrm{W}$ & Windy \\
\hline $\mathrm{N}$ & Unremarked \\
\hline $\mathrm{C}$ & Cattle nearby \\
\hline $\mathrm{L}$ & Normal laboratory \\
\hline \multicolumn{2}{|r|}{ Sample matrix } \\
\hline DI & Deionized water from USGS water systems in Denver \\
\hline TAP & Municipal tap water \\
\hline IBW & Certified Inorganic Blank Water from USGS laboratory in Ocala, Florida \\
\hline ST & Sterile buffered water (for bacteria-sample blanks) \\
\hline \multicolumn{2}{|r|}{ Matrix source } \\
\hline $\mathrm{C}$ & Plastic carboy, reused each trip \\
\hline $\mathrm{J}$ & Plastic 1-gallon jug from Ocala laboratory, used one time only \\
\hline $\mathrm{L}$ & USGS laboratory spigot \\
\hline BJ & Blue plastic 5-gallon jug, reused each trip \\
\hline $\mathrm{U}$ & Undocumented USGS deionized-water source \\
\hline \multicolumn{2}{|r|}{ 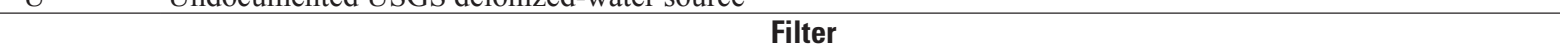 } \\
\hline $\mathrm{P}$ & 0.45 micron cellulose nitrate disposable filter in reused plate stand \\
\hline $\mathrm{C}$ & 0.45 micron cellulose nitrate disposable filter factory-sealed in plastic capsule \\
\hline $\mathrm{N}$ & None used \\
\hline $\mathrm{U}$ & Not documented \\
\hline $\mathrm{D}$ & 0.45 micron nylon-disc filter for syringe \\
\hline \multicolumn{2}{|r|}{0.45 imll } \\
\hline G1 & Submersible pump (Grundfos) attached to polyethelene hose, used 1993-1996 \\
\hline GP & Assorted project-owned peristaltic pumps (Geopump) \\
\hline $\mathrm{G} 2$ & Submersible pump (Grundfos) attached to teflon-lined polyethelene hose, used 1996-1998 \\
\hline G3 & Submersible pump (Grundfos) with independent, quick-connected polyethelene hose sections, used 1998-2001 \\
\hline G4 & Submersible pump (Grundfos) attached to teflon-lined polyethelene hose, used 1998-1999 \\
\hline G5 & USGS Arsenal project's submersible pump (Grundfos) attached to polyethelene hose, used January 1998, only \\
\hline G6 & Submersible pump (Grundfos) attached to teflon-lined polyethelene hose, used 1999-2003 \\
\hline B1 & Polyvinyl chloride (PVC) bailer, 4 feet long, with rusty metal fasteners and old, reused nylon rope \\
\hline $\mathrm{B} 2$ & Disposable polyethylene bailer with nylon rope and clean stainless-steel fastener \\
\hline B3 & Brass bailer tied to old polyester-cotton rope, used for bacteria sampling only \\
\hline B4 & Autoclaved, polyethylene disposable bailer, clean stainless-steel fastener, and teflon-coated cable \\
\hline $\mathrm{N}$ & None used \\
\hline $\mathrm{U}$ & Not documented \\
\hline
\end{tabular}


Appendix 6. Codes used in reporting information and chemical data (appendix 7) for blank water-quality samples collected near Deer Trail, Colorado, 1993 through 1999.-Continued

[USGS, U.S. Geological Survey]

\begin{tabular}{cl}
\hline & \\
\hline A & Dedicated to unique site or use \\
B & Reused, cleaned between sites with deionized water only \\
U & Not documented \\
C & Reused, cleaned between sites with 5-percent hydrochloric acid \\
D & Disposed of after each use \\
E & Reused, cleaned between sites with laboratory-grade nonphosphate detergent and deionized water \\
F & New \\
N & None used \\
\hline & No "parts per billion" preparation, equipment, or packing \\
\hline T & Team 1 "parts per billion" preparation, equipment, and packing \\
2 & Team 2 "parts per billion" preparation, equipment, and packing \\
\hline K & USGS Western Region Field Manual \\
B & Bailed into a rinsed pail when pump malfunctioned method \\
PPB & USGS "Parts per Billion" method (Horowitz and others, 1994; U.S. Geological Survey, variously dated) \\
S & Source transfer, not a regular sampling method \\
F & Other USGS project sampling method (not PPB) \\
J & Pore-water method (Tindall and Vencill, 1995, p. 47) \\
\hline & \\
\hline Metro & Metro Wastewater Reclamation District Laboratory in Denver, Colorado \\
District & \\
NWQL & USGS National Water Quality Laboratory in Denver, Colorado \\
\hline
\end{tabular}


Appendix 7. Information and chemical data for blank water-quality samples collected near Deer Trail, Colorado, 1993 through 1999.

[Codes are explained in appendix 6; other abbreviations are listed in appendix 2; $\mathrm{mg} / \mathrm{L}$, milligrams per liter; $\mu \mathrm{S} / \mathrm{cm}$, microsiemens per centimeter at 25 degrees Celsius; $\mu \mathrm{g} / \mathrm{L}$, micrograms per liter; all data from filtered samples unless otherwise indicated; <, less than; --, no data]

\begin{tabular}{|c|c|c|c|c|c|c|c|c|c|c|c|c|c|c|c|c|c|c|}
\hline $\begin{array}{l}\text { Sampling } \\
\text { type }\end{array}$ & $\begin{array}{c}\text { Blank } \\
\text { number }\end{array}$ & $\begin{array}{l}\text { Sample } \\
\text { date }\end{array}$ & $\begin{array}{l}\text { Type of } \\
\text { blank }\end{array}$ & $\begin{array}{c}\text { Sample } \\
\text { sub-type }\end{array}$ & $\begin{array}{l}\text { Sample } \\
\text { site }\end{array}$ & $\begin{array}{l}\text { Ambient } \\
\text { condition }\end{array}$ & $\begin{array}{c}\text { Sample } \\
\text { matrix }\end{array}$ & $\begin{array}{l}\text { Matrix } \\
\text { source }\end{array}$ & Filter & Pump & Tubing & $\begin{array}{l}\text { Sampling } \\
\text { (team) } \\
\text { equipment }\end{array}$ & $\begin{array}{l}\text { Sample } \\
\text { method }\end{array}$ & $\begin{array}{l}\text { Analyzing } \\
\text { laboratory }\end{array}$ & $\begin{array}{c}\text { Alk, lab, } \\
\text { mg/L }\end{array}$ & $\begin{array}{c}\text { Hard } \\
\left(\mathrm{CaCO}_{3}\right), \\
\mathrm{mg} / \mathrm{L}\end{array}$ & $\begin{array}{l}\mathrm{pH}, \\
\text { lab }\end{array}$ & $\begin{array}{c}\text { SC, } \\
\text { lab, } \\
\mu S / c m\end{array}$ \\
\hline 2 & 1 & 7/20/1994 & FB & F & D9 & $\mathrm{R}$ & DI & BJ & $\mathrm{P}$ & G1 & $\mathrm{U}$ & 0 & $\mathrm{~K}$ & Metro District & 2 & $<10$ & -- & -- \\
\hline 2 & 2 & $10 / 3 / 1994$ & $\mathrm{FB}$ & $\mathrm{F}$ & D5 & $\mathrm{B}$ & DI & BJ & $\mathrm{N}$ & $\mathrm{N}$ & $\mathrm{N}$ & 0 & $\mathrm{~K}$ & Metro District & 0 & $<10$ & -- & -- \\
\hline 2 & 3 & $10 / 3 / 1994$ & FB & $\mathrm{F}$ & D5 & $\mathrm{B}$ & DI & BJ & $\mathrm{P}$ & GP & $\mathrm{E}$ & 0 & $\mathrm{~K}$ & Metro District & 0 & $<10$ & -- & -- \\
\hline 2 & 4 & $10 / 4 / 1994$ & FB & $\mathrm{E}$ & D2 & W & DI & BJ & $\mathrm{N}$ & $\mathrm{N}$ & $\mathrm{N}$ & 0 & $\mathrm{~K}$ & Metro District & 0 & $<10$ & -- & -- \\
\hline 2 & 5 & $10 / 4 / 1994$ & FB & $\mathrm{E}$ & D2 & W & TAP & BJ & $\mathrm{N}$ & $\mathrm{N}$ & $\mathrm{N}$ & 0 & $\mathrm{~K}$ & Metro District & 62 & 114 & -- & -- \\
\hline 2 & 6 & $7 / 12 / 1995$ & FB & $\mathrm{F}$ & $\mathrm{D} 25$ & $\mathrm{R}$ & DI & BJ & $\mathrm{U}$ & $\mathrm{U}$ & $\mathrm{U}$ & 0 & $\mathrm{~K}$ & Metro District & 0 & $<10$ & -- & -- \\
\hline 2 & 7 & 9/27/1995 & FB & $\mathrm{F}$ & D2 & W & DI & $\mathrm{C}$ & $\mathrm{P}$ & GP & $\mathrm{E}$ & 0 & $\mathrm{~K}$ & Metro District & 0 & $<10$ & -- & -- \\
\hline 2 & 8 & 9/28/1995 & $\mathrm{FB}$ & $\mathrm{F}$ & D9 & $\mathrm{R}$ & DI & $\mathrm{C}$ & $\mathrm{N}$ & B1 & $\mathrm{N}$ & 0 & B & Metro District & 0 & $<10$ & -- & -- \\
\hline 2 & 9 & $12 / 14 / 1995$ & FB & $\mathrm{F}$ & D16 & $\mathrm{W}$ & IBW & $\mathrm{J}$ & $\mathrm{C}$ & GP & A & 1 & PPB & Metro District & 0 & $<10$ & -- & -- \\
\hline 2 & 10 & $12 / 15 / 1995$ & EB & $\mathrm{E}$ & $\mathrm{CD}$ & $\mathrm{L}$ & IBW & $\mathrm{J}$ & $\mathrm{C}$ & G1 & $\mathrm{E}$ & 2 & PPB & Metro District & 0 & $<10$ & -- & -- \\
\hline 2 & 11 & 3/8/1996 & FB & $\mathrm{F}$ & D33 & W & IBW & $\mathrm{J}$ & $\mathrm{C}$ & GP & A & 2 & $\mathrm{~F}$ & Metro District & 8 & $<10$ & -- & -- \\
\hline 2 & 12 & $3 / 11 / 1996$ & $\mathrm{~EB}$ & $\mathrm{E}$ & $\mathrm{CD}$ & $\mathrm{L}$ & IBW & J & $\mathrm{C}$ & G1 & A & 2 & $\mathrm{~F}$ & NWQL & -- & 0 & -- & -- \\
\hline 2 & 13 & $6 / 10 / 1996$ & EB & $\mathrm{E}$ & $\mathrm{CD}$ & $\mathrm{L}$ & IBW & $\mathrm{J}$ & $\mathrm{C}$ & $\mathrm{G} 2$ & $\mathrm{~F}$ & 2 & $\mathrm{~F}$ & NWQL & -- & 1 & -- & -- \\
\hline 2 & 14 & $6 / 12 / 1996$ & FB & $\mathrm{F}$ & D2 & $\mathrm{N}$ & IBW & $\mathrm{J}$ & $\mathrm{C}$ & GP & $\mathrm{F}$ & 2 & $\mathrm{~F}$ & Metro District & 5 & $<10$ & -- & -- \\
\hline 2 & 15 & $6 / 14 / 1996$ & FB & $\mathrm{F}$ & D9 & $\mathrm{R}$ & IBW & $\mathrm{J}$ & $\mathrm{C}$ & $\mathrm{B} 2$ & $\mathrm{~F}$ & 1 & B & Metro District & 4 & $<10$ & -- & -- \\
\hline 2 & 16 & $6 / 14 / 1996$ & FB & A & D9 & $\mathrm{R}$ & DI & $\mathrm{C}$ & $\mathrm{N}$ & $\mathrm{N}$ & $\mathrm{N}$ & 1 & $\mathrm{~S}$ & Metro District & 7 & $<10$ & -- & -- \\
\hline 2 & 17 & $8 / 21 / 1996$ & FB & $\mathrm{F}$ & D30 & $\mathrm{N}$ & IBW & $\mathrm{J}$ & $\mathrm{C}$ & GP & A & 2 & $\mathrm{~F}$ & Metro District & 0 & $<10$ & -- & -- \\
\hline 2 & 18 & $8 / 22 / 1996$ & FB & $\mathrm{F}$ & D20 & W & IBW & $\mathrm{J}$ & $\mathrm{C}$ & GP & A & 1 & PPB & Metro District & 0 & $<10$ & -- & -- \\
\hline 2 & 19 & $8 / 23 / 1996$ & EB & $\mathrm{E}$ & $\mathrm{CD}$ & $\mathrm{L}$ & DI & $\mathrm{C}$ & $\mathrm{N}$ & $\mathrm{N}$ & A & 1 & $\mathrm{~S}$ & NWQL & -- & -- & 5.6 & 0.800 \\
\hline 2 & 20 & $8 / 23 / 1996$ & EB & $\mathrm{E}$ & $\mathrm{CD}$ & $\mathrm{L}$ & IBW & $\mathrm{J}$ & $\mathrm{N}$ & $\mathrm{N}$ & $\mathrm{N}$ & 1 & $\mathrm{~S}$ & NWQL & -- & -- & 5.6 & 1 \\
\hline 2 & 21 & $8 / 28 / 1996$ & FB & F & $\mathrm{L}$ & W & IBW & $\mathrm{J}$ & $\mathrm{C}$ & GP & $\mathrm{C}$ & 1 & PPB & Metro District & 0 & $<10$ & -- & -- \\
\hline 2 & 22 & $8 / 29 / 1996$ & EB & $\mathrm{E}$ & $\mathrm{CD}$ & $\mathrm{L}$ & IBW & $\mathrm{J}$ & $\mathrm{C}$ & $\mathrm{G} 2$ & A & 2 & $\mathrm{~F}$ & NWQL & -- & 0 & 5.8 & 1 \\
\hline 2 & 23 & $8 / 29 / 1996$ & EB & A & $\mathrm{CD}$ & $\mathrm{L}$ & IBW & $\mathrm{J}$ & $\mathrm{N}$ & $\mathrm{N}$ & $\mathrm{N}$ & 2 & PPB & NWQL & -- & -- & -- & -- \\
\hline 2 & 24 & $11 / 8 / 1996$ & FB & $\mathrm{F}$ & D25 & $\mathrm{R}$ & IBW & $\mathrm{J}$ & $\mathrm{C}$ & GP & A & 1 & PPB & Metro District & 0 & $<10$ & -- & -- \\
\hline 2 & 25 & $11 / 8 / 1996$ & FB & $\mathrm{F}$ & D5 & $\mathrm{W}$ & IBW & $\mathrm{J}$ & $\mathrm{C}$ & GP & A & 2 & $\mathrm{~F}$ & Metro District & 0 & $<10$ & -- & -- \\
\hline 2 & 26 & $2 / 25 / 1997$ & FB & $\mathrm{F}$ & D30 & $\mathrm{N}$ & IBW & J & $\mathrm{C}$ & GP & A & 2 & $\mathrm{~F}$ & Metro District & 0 & $<10$ & -- & -- \\
\hline 2 & 27 & $2 / 26 / 1997$ & FB & $\mathrm{F}$ & D15 & W & IBW & $\mathrm{J}$ & $\mathrm{C}$ & GP & A & 1 & PPB & Metro District & 0 & $<10$ & -- & -- \\
\hline 2 & 28 & $6 / 4 / 1997$ & FB & $\mathrm{F}$ & D17 & W & IBW & J & $\mathrm{C}$ & GP & A & 1 & PPB & Metro District & 8 & $<10$ & -- & -- \\
\hline 2 & 29 & 6/5/1997 & EB & $\mathrm{E}$ & $\mathrm{CD}$ & $\mathrm{L}$ & IBW & $\mathrm{J}$ & $\mathrm{C}$ & $\mathrm{G} 2$ & A & 2 & $\mathrm{~F}$ & NWQL & -- & 0 & 6.1 & 1.2 \\
\hline 2 & 30 & 8/27/1997 & FB & $\mathrm{F}$ & D25 & $\mathrm{R}$ & IBW & $\mathrm{J}$ & $\mathrm{C}$ & GP & A & 1 & PPB & Metro District & 0 & $<10$ & -- & -- \\
\hline 2 & 31 & 8/26/1997 & FB & $\mathrm{F}$ & D33 & $\mathrm{N}$ & IBW & $\mathrm{J}$ & $\mathrm{C}$ & GP & A & 2 & $\mathrm{~F}$ & Metro District & 0 & $<10$ & -- & -- \\
\hline 2 & 32 & $8 / 29 / 1997$ & FB & $\mathrm{F}$ & $\mathrm{L}$ & $\mathrm{N}$ & IBW & $\mathrm{J}$ & $\mathrm{C}$ & GP & $\mathrm{C}$ & 1 & PPB & Metro District & 0 & $<10$ & -- & -- \\
\hline 2 & 33 & 11/19/1997 & FB & $\mathrm{F}$ & D2 & W & IBW & $\mathrm{J}$ & $\mathrm{C}$ & GP & A & 2 & $\mathrm{~F}$ & Metro District & 0 & $<10$ & -- & -- \\
\hline 2 & 34 & $11 / 20 / 1997$ & FB & $\mathrm{F}$ & D15 & $\mathrm{N}$ & IBW & $\mathrm{J}$ & $\mathrm{C}$ & GP & A & 1 & PPB & Metro District & 0 & $<10$ & -- & -- \\
\hline 2 & 35 & 11/21/1997 & $\mathrm{EB}$ & E & $\mathrm{CD}$ & $\mathrm{L}$ & IBW & $\mathrm{J}$ & $\mathrm{C}$ & $\mathrm{G} 2$ & A & 2 & PPB & NWQL & -- & 0 & 5.5 & 1.5 \\
\hline 2 & 36 & $12 / 4 / 1997$ & $\mathrm{~EB}$ & $\mathrm{E}$ & $\mathrm{CD}$ & $\mathrm{L}$ & DI & $\mathrm{C}$ & $\mathrm{C}$ & GP & A & 1 & PPB & NWQL & -- & -- & 5.7 & 1.5 \\
\hline 2 & 37 & $1 / 8 / 1998$ & FB & $\mathrm{F}$ & D25 & $\mathrm{R}$ & IBW & $\mathrm{J}$ & $\mathrm{C}$ & GP & A & 1 & PPB & Metro District & 11 & $<10$ & -- & -- \\
\hline 2 & 38 & $1 / 8 / 1998$ & FB & $\mathrm{F}$ & D23 & $\mathrm{N}$ & IBW & $\mathrm{J}$ & $\mathrm{C}$ & GP & A & 2 & PPB & Metro District & 5 & $<10$ & -- & -- \\
\hline 2 & 39 & $1 / 15 / 1998$ & EB & $\mathrm{E}$ & $\mathrm{CD}$ & $\mathrm{L}$ & IBW & $\mathrm{J}$ & $\mathrm{C}$ & G5 & A & 2 & PPB & NWQL & -- & 0 & 5.9 & 1.4 \\
\hline 2 & 40 & $3 / 27 / 1998$ & EB & $\mathrm{E}$ & $\mathrm{CD}$ & $\mathrm{L}$ & DI & $\mathrm{L}$ & $\mathrm{C}$ & G4 & $\mathrm{F}$ & 2 & PPB & NWQL & -- & 0 & 5.7 & 1.0 \\
\hline 2 & 41 & $4 / 16 / 1998$ & FB & $\mathrm{F}$ & D25 & $\mathrm{R}$ & IBW & $\mathrm{J}$ & $\mathrm{C}$ & GP & A & 1 & PPB & Metro District & 0 & $<10$ & -- & -- \\
\hline 2 & 42 & $4 / 16 / 1998$ & FB & $\mathrm{F}$ & D23 & $\mathrm{N}$ & IBW & $\mathrm{J}$ & $\mathrm{C}$ & GP & A & 2 & PPB & Metro District & 0 & $<10$ & -- & -- \\
\hline 2 & 43 & $7 / 15 / 1998$ & FB & $\mathrm{F}$ & D5 & W & IBW & $\mathrm{J}$ & $\mathrm{C}$ & GP & A & 1 & PPB & Metro District & 0 & $<10$ & -- & -- \\
\hline 2 & 44 & 7/16/1998 & FB & $\mathrm{F}$ & D6 & $\mathrm{W}$ & IBW & $\mathrm{J}$ & $\mathrm{C}$ & GP & A & 2 & PPB & Metro District & 0 & $<10$ & -- & -- \\
\hline 2 & 45 & $7 / 17 / 1998$ & $\mathrm{~EB}$ & E & $\mathrm{CD}$ & $\mathrm{L}$ & DI & BJ & $\mathrm{N}$ & $\mathrm{N}$ & $\mathrm{N}$ & 2 & $\mathrm{~S}$ & NWQL & -- & -- & 5.65 & 1.6 \\
\hline 2 & 46 & $7 / 21 / 1998$ & EB & $\mathrm{E}$ & $\mathrm{CD}$ & $\mathrm{L}$ & DI & $\mathrm{C}$ & $\mathrm{N}$ & $\mathrm{N}$ & A & 1 & $\mathrm{~S}$ & NWQL & -- & -- & 5.50 & 1.2 \\
\hline 2 & 47 & $7 / 21 / 1998$ & EB & $\mathrm{E}$ & $\mathrm{CD}$ & $\mathrm{L}$ & DI & $\mathrm{C}$ & $\mathrm{N}$ & $\mathrm{N}$ & A & 2 & $\mathrm{~S}$ & NWQL & -- & -- & 5.58 & 1.2 \\
\hline 2 & 48 & 7/13/1999 & FB & $\mathrm{F}$ & D23 & $\mathrm{W}$ & IBW & $\mathrm{J}$ & $\mathrm{C}$ & GP & A & 1 & PPB & NWQL & 1.9 & -- & 7.7 & 2 \\
\hline 3 & 49 & $4 / 22 / 1994$ & $\mathrm{~EB}$ & E & $\mathrm{CD}$ & $\mathrm{N}$ & DI & $\mathrm{U}$ & $\mathrm{D}$ & $\mathrm{N}$ & $\mathrm{N}$ & 0 & $\mathrm{~J}$ & Metro District & -- & -- & -- & -- \\
\hline
\end{tabular}


Appendix 7. Information and chemical data for blank water-quality samples collected near Deer Trail, Colorado, 1993 through 1999.—Continued

[Codes are explained in appendix 6; other abbreviations are listed in appendix $2 ; \mathrm{mg} / \mathrm{L}$, milligrams per liter; $\mu \mathrm{S} / \mathrm{cm}$, microsiemens per centimeter at $25 \mathrm{degrees}$ Celsius; $\mu \mathrm{g} / \mathrm{L}$, micrograms per liter; all data from filtered samples unless otherwise indicated; $<$, less than; --, no data]

\begin{tabular}{|c|c|c|c|c|c|c|c|c|c|c|c|c|c|c|c|}
\hline $\begin{array}{c}\text { Sampling } \\
\text { type }\end{array}$ & $\begin{array}{c}\text { Blank } \\
\text { number }\end{array}$ & $\begin{array}{c}\text { Sample } \\
\text { date }\end{array}$ & $\begin{array}{c}\text { TDS (Calc), } \\
\text { mg/L }\end{array}$ & $\begin{array}{c}\text { TDS (ROE), } \\
\text { mg/L }\end{array}$ & $\begin{array}{l}\mathrm{Br}, \\
\mathrm{mg} / \mathrm{L}\end{array}$ & $\begin{array}{c}\mathrm{Ca}, \\
\mathrm{mg} / \mathrm{L}\end{array}$ & $\begin{array}{c}\mathrm{Cl}, \\
\mathrm{mg} / \mathrm{L}\end{array}$ & $\begin{array}{c}\mathbf{F} \\
\mathrm{mg} / \mathrm{L}\end{array}$ & $\begin{array}{c}\mathrm{K}, \\
\mathrm{mg} / \mathrm{L}\end{array}$ & $\begin{array}{c}\mathrm{Mg}, \\
\mathrm{mg} / \mathrm{L}\end{array}$ & $\begin{array}{l}\mathrm{Na}, \\
\mathrm{mg} / \mathrm{L}\end{array}$ & $\begin{array}{l}\mathrm{SiO}_{2^{\prime}} \\
\mathrm{mg} / \mathrm{L}\end{array}$ & $\begin{array}{l}\mathrm{SO}_{4^{\prime}} \\
\mathrm{mg} / \mathrm{L}\end{array}$ & $\begin{array}{c}\mathrm{NH}_{3}+\mathrm{Org} \mathrm{N}-\mathrm{N}, \\
\text { diss, } \mathrm{mg} / \mathrm{L}\end{array}$ & $\begin{array}{c}\mathrm{NH}_{3}+\mathrm{Org} \mathrm{N}-\mathrm{N}, \\
\text { tot, } \mathrm{mg} / \mathrm{L}\end{array}$ \\
\hline 2 & 1 & $7 / 20 / 1994$ & -- & $<10$ & -- & $<5$ & $<1$ & -- & 0.1 & $<5$ & $<5$ & -- & $<3$ & 0.3 & -- \\
\hline 2 & 2 & $10 / 3 / 1994$ & -- & $<10$ & -- & $<2$ & $<1$ & -- & $<0.1$ & $<2$ & $<2$ & -- & $<3$ & $<0.3$ & -- \\
\hline 2 & 3 & $10 / 3 / 1994$ & -- & $<10$ & -- & $<1$ & $<1$ & -- & $<0.1$ & $<1$ & $<1$ & -- & $<3$ & $<0.3$ & -- \\
\hline 2 & 4 & $10 / 4 / 1994$ & -- & 53 & -- & $<1$ & $<1$ & -- & 0.2 & $<1$ & $<1$ & -- & $<3$ & $<0.3$ & -- \\
\hline 2 & 5 & $10 / 4 / 1994$ & -- & 209 & -- & 32 & 24 & -- & 2.1 & 7 & 19 & -- & 55 & 0.5 & -- \\
\hline 2 & 6 & $7 / 12 / 1995$ & -- & $<10$ & -- & $<1$ & $<1$ & -- & $<0.1$ & $<1$ & $<1$ & -- & 7 & $<0.3$ & -- \\
\hline 2 & 7 & 9/27/1995 & -- & $<10$ & -- & $<1$ & $<1$ & -- & $<0.1$ & $<1$ & $<1$ & -- & 10 & $<0.3$ & -- \\
\hline 2 & 8 & $9 / 28 / 1995$ & -- & $<10$ & -- & $<1$ & $<1$ & -- & $<0.1$ & $<1$ & $<1$ & -- & 9 & $<0.3$ & -- \\
\hline 2 & 9 & $12 / 14 / 1995$ & -- & $<10$ & -- & $<1$ & $<1$ & -- & $<0.1$ & $<1$ & $<1$ & -- & 4 & $<0.3$ & -- \\
\hline 2 & 10 & $12 / 15 / 1995$ & -- & $<10$ & -- & $<1$ & $<1$ & -- & $<0.1$ & $<1$ & $<1$ & -- & $<3$ & $<0.3$ & -- \\
\hline 2 & 11 & $3 / 8 / 1996$ & -- & $<10$ & -- & $<1$ & $<1$ & -- & $<0.1$ & $<1$ & $<1$ & -- & $<3$ & $<0.3$ & -- \\
\hline 2 & 12 & $3 / 11 / 1996$ & -- & -- & -- & 0.05 & -- & -- & -- & 0.008 & $<0.03$ & $<0.02$ & -- & -- & -- \\
\hline 2 & 13 & $6 / 10 / 1996$ & -- & -- & -- & 0.34 & -- & -- & -- & 0.01 & 0.05 & 0.04 & -- & -- & -- \\
\hline 2 & 14 & $6 / 12 / 1996$ & -- & $<10$ & -- & $<1$ & $<1$ & -- & $<0.1$ & $<1$ & $<1$ & -- & $<3$ & $<0.3$ & -- \\
\hline 2 & 15 & $6 / 14 / 1996$ & -- & $<10$ & -- & $<1$ & $<1$ & -- & $<0.1$ & $<1$ & $<1$ & -- & $<3$ & $<0.3$ & -- \\
\hline 2 & 16 & $6 / 14 / 1996$ & -- & $<10$ & -- & $<1$ & $<1$ & -- & $<0.1$ & $<1$ & $<1$ & -- & $<3$ & $<0.3$ & -- \\
\hline 2 & 17 & $8 / 21 / 1996$ & -- & $<10$ & -- & $<1$ & $<1$ & -- & $<0.1$ & $<1$ & $<1$ & -- & $<3$ & $<0.3$ & -- \\
\hline 2 & 18 & $8 / 22 / 1996$ & -- & $<10$ & -- & $<1$ & $<1$ & -- & $<0.1$ & $<1$ & $<1$ & -- & 4 & $<0.3$ & -- \\
\hline 2 & 19 & $8 / 23 / 1996$ & -- & -- & $<0.010$ & $<0.002$ & $<0.01$ & $<0.01$ & -- & $<0.001$ & $<0.03$ & $<0.020$ & $<0.01$ & -- & -- \\
\hline 2 & 20 & $8 / 23 / 1996$ & -- & -- & $<0.010$ & $<0.002$ & $<0.01$ & $<0.01$ & -- & $<0.001$ & $<0.03$ & $<0.020$ & $<0.01$ & -- & -- \\
\hline 2 & 21 & $8 / 28 / 1996$ & -- & $<10$ & -- & $<1$ & $<1$ & -- & $<0.1$ & $<1$ & $<1$ & -- & 4 & 0.7 & -- \\
\hline 2 & 22 & $8 / 29 / 1996$ & -- & -- & $<0.010$ & 0.04 & $<0.01$ & $<0.01$ & -- & 0.004 & $<0.03$ & 0.025 & 0.04 & -- & -- \\
\hline 2 & 23 & $8 / 29 / 1996$ & -- & -- & -- & $<0.002$ & -- & -- & -- & $<0.001$ & $<0.03$ & $<0.020$ & -- & -- & -- \\
\hline 2 & 24 & $11 / 8 / 1996$ & -- & 66 & -- & $<1$ & $<1$ & -- & $<0.1$ & $<1$ & $<1$ & -- & $<3$ & $<0.3$ & -- \\
\hline 2 & 25 & $11 / 8 / 1996$ & -- & 36 & -- & $<1$ & $<1$ & -- & $<0.1$ & $<1$ & $<1$ & -- & $<3$ & $<0.3$ & -- \\
\hline 2 & 26 & $2 / 25 / 1997$ & -- & 11 & -- & $<1$ & $<1$ & -- & $<0.1$ & $<1$ & $<1$ & -- & $<3$ & $<0.3$ & -- \\
\hline 2 & 27 & $2 / 26 / 1997$ & -- & 37 & -- & $<1$ & $<1$ & -- & $<0.1$ & $<1$ & $<1$ & -- & $<3$ & $<0.3$ & -- \\
\hline 2 & 28 & $6 / 4 / 1997$ & -- & $<10$ & -- & $<1$ & 1 & -- & $<0.1$ & $<1$ & $<1$ & -- & $<3$ & $<0.3$ & -- \\
\hline 2 & 29 & $6 / 5 / 1997$ & -- & -- & $<0.01$ & 0.076 & $<0.01$ & $<0.01$ & -- & 0.009 & $<0.025$ & $<0.020$ & $<0.01$ & $<0.20$ & -- \\
\hline 2 & 30 & $8 / 27 / 1997$ & -- & $<10$ & -- & $<1$ & $<1$ & -- & $<0.1$ & $<1$ & $<1$ & -- & $<3$ & $<0.3$ & -- \\
\hline 2 & 31 & $8 / 26 / 1997$ & -- & 14 & -- & $<1$ & $<1$ & -- & $<0.1$ & $<1$ & $<1$ & -- & 4 & $<0.3$ & -- \\
\hline 2 & 32 & $8 / 29 / 1997$ & -- & 3 & -- & $<1$ & $<1$ & -- & $<0.1$ & $<1$ & $<1$ & -- & $<3$ & $<0.3$ & -- \\
\hline 2 & 33 & $11 / 19 / 1997$ & -- & $<10$ & -- & $<1$ & 1 & -- & $<0.1$ & 1 & 1 & -- & $<3$ & $<0.3$ & -- \\
\hline 2 & 34 & $11 / 20 / 1997$ & -- & $<10$ & -- & $<1$ & 1 & -- & $<0.1$ & $<1$ & $<1$ & -- & $<3$ & $<0.3$ & -- \\
\hline 2 & 35 & $11 / 21 / 1997$ & -- & -- & $<0.01$ & 0.047 & 0.02 & $<0.01$ & -- & 0.007 & $<0.025$ & 0.047 & 0.03 & $<0.10$ & -- \\
\hline 2 & 36 & 12/4/1997 & -- & -- & $<0.01$ & 0.002 & $<0.01$ & $<0.01$ & -- & $<0.001$ & $<0.025$ & $<0.020$ & $<0.01$ & $<0.10$ & -- \\
\hline 2 & 37 & $1 / 8 / 1998$ & -- & $<10$ & -- & $<1$ & $<1$ & -- & $<0.1$ & $<1$ & $<1$ & -- & $<3$ & $<0.3$ & -- \\
\hline 2 & 38 & $1 / 8 / 1998$ & -- & $<10$ & -- & $<1$ & $<1$ & -- & $<0.1$ & $<1$ & $<1$ & -- & $<3$ & $<0.3$ & -- \\
\hline 2 & 39 & $1 / 15 / 1998$ & -- & -- & $<0.01$ & 0.044 & 0.02 & $<0.01$ & -- & 0.002 & $<0.025$ & $<0.020$ & $<0.01$ & $<0.10$ & -- \\
\hline 2 & 40 & $3 / 27 / 1998$ & -- & -- & $<0.01$ & 0.010 & $<0.01$ & $<0.01$ & -- & 0.001 & $<0.025$ & $<0.020$ & $<0.01$ & $<0.10$ & -- \\
\hline 2 & 41 & $4 / 16 / 1998$ & -- & 63 & -- & $<1$ & $<1$ & -- & $<0.1$ & $<1$ & $<1$ & -- & $<3$ & $<0.3$ & -- \\
\hline 2 & 42 & $4 / 16 / 1998$ & -- & $<10$ & -- & $<1$ & $<1$ & -- & $<0.1$ & $<1$ & $<1$ & -- & $<3$ & $<0.3$ & -- \\
\hline 2 & 43 & $7 / 15 / 1998$ & -- & $<10$ & -- & $<1$ & $<1$ & -- & $<0.1$ & $<1$ & $<1$ & -- & $<3$ & 0.3 & -- \\
\hline 2 & 44 & $7 / 16 / 1998$ & -- & $<10$ & -- & $<1$ & $<1$ & -- & $<0.1$ & $<1$ & $<1$ & -- & $<3$ & 0.4 & -- \\
\hline 2 & 45 & $7 / 17 / 1998$ & -- & -- & $<0.01$ & $<0.002$ & 0.39 & 0.02 & -- & $<0.001$ & $<0.025$ & $<0.020$ & $<0.1$ & $<0.10$ & -- \\
\hline 2 & 46 & $7 / 21 / 1998$ & -- & -- & $<0.01$ & $<0.002$ & $<0.01$ & 0.03 & -- & $<0.001$ & $<0.025$ & $<0.020$ & 3.3 & $<0.10$ & -- \\
\hline 2 & 47 & $7 / 21 / 1998$ & -- & -- & $<0.01$ & $<0.002$ & 0.26 & 0.03 & -- & $<0.001$ & $<0.025$ & $<0.020$ & 11 & $<0.10$ & -- \\
\hline 2 & 48 & $7 / 13 / 1999$ & $<10$ & $<10$ & $<0.01$ & 00.01 & $<0.1$ & $<0.1$ & $<0.1$ & 0.01 & $\mathrm{e} 0.04$ & $<0.05$ & $<0.1$ & $<0.1$ & $<0.1$ \\
\hline 3 & 49 & $4 / 22 / 1994$ & -- & -- & -- & $<1$ & $<1$ & -- & $<1$ & $<1$ & $<1$ & -- & $<3$ & $<0.3$ & -- \\
\hline
\end{tabular}


Appendix 7. Information and chemical data for blank water-quality samples collected near Deer Trail, Colorado, 1993 through 1999._Continued

[Codes are explained in appendix 6; other abbreviations are listed in appendix 2; $\mathrm{mg} / \mathrm{L}$, milligrams per liter; $\mu \mathrm{S} / \mathrm{cm}$, microsiemens per centimeter at 25 degrees Celsius; $\mu \mathrm{g} / \mathrm{L}$, micrograms per liter; all data from filtered samples unless otherwise indicated; <, less than; --, no data]

\begin{tabular}{|c|c|c|c|c|c|c|c|c|c|c|c|c|c|c|c|c|}
\hline $\begin{array}{c}\text { Sampling } \\
\text { type }\end{array}$ & $\begin{array}{c}\text { Blank } \\
\text { number }\end{array}$ & $\begin{array}{c}\text { Sampling } \\
\text { date }\end{array}$ & $\begin{array}{c}\mathrm{NH}_{3}-\mathrm{N}, \\
\mathrm{mg} / \mathrm{L}\end{array}$ & $\begin{array}{c}\mathrm{NO}_{2}-\mathrm{N}, \\
\mathrm{mg} / \mathrm{L}\end{array}$ & $\begin{array}{c}\mathrm{NO}_{2}+\mathrm{NO}_{3}-\mathrm{N} \\
\mathrm{mg} / \mathrm{L}\end{array}$ & $\begin{array}{c}\mathrm{NO}_{3}-\mathrm{N}, \\
\mathrm{mg} / \mathrm{L}\end{array}$ & $\begin{array}{l}\text { P, diss, } \\
\mathrm{mg} / \mathrm{L}\end{array}$ & $\begin{array}{l}\mathrm{P}, \text { tot, } \\
\mathrm{mg} / \mathrm{L}\end{array}$ & $\begin{array}{c}\mathrm{PO}_{4}-\mathrm{P}, \\
\mathrm{mg} / \mathrm{L}\end{array}$ & $\begin{array}{l}\mathrm{Ag}, \\
\mu \mathrm{g} / \mathrm{L}\end{array}$ & $\begin{array}{c}\text { Al, } \\
\mu \mathrm{g} / \mathrm{L}\end{array}$ & $\begin{array}{c}\text { B, } \\
\mu g / L\end{array}$ & $\begin{array}{c}\mathrm{Ba}, \\
\mu \mathrm{g} / \mathrm{L}\end{array}$ & $\begin{array}{c}\mathrm{Be}, \\
\mu \mathrm{g} / \mathrm{L}\end{array}$ & $\begin{array}{c}\text { Cd, } \\
\mu \mathrm{g} / \mathrm{L}\end{array}$ & $\begin{array}{c}\text { Co, } \\
\mu \mathrm{g} / \mathrm{L}\end{array}$ \\
\hline 2 & 1 & $7 / 20 / 1994$ & $<0.1$ & $<0.02$ & $<0.02$ & -- & -- & -- & $<0.02$ & -- & -- & -- & -- & -- & $<0.2$ & -- \\
\hline 2 & 2 & $10 / 3 / 1994$ & $<0.1$ & $<0.02$ & $<0.02$ & -- & -- & -- & $<0.02$ & -- & -- & -- & -- & -- & $<0.2$ & -- \\
\hline 2 & 3 & $10 / 3 / 1994$ & $<0.1$ & $<0.02$ & $<0.02$ & -- & -- & -- & $<0.02$ & -- & -- & -- & -- & -- & $<0.2$ & -- \\
\hline 2 & 4 & $10 / 4 / 1994$ & $<0.1$ & $<0.02$ & $<0.02$ & -- & -- & -- & $<0.02$ & -- & -- & -- & -- & -- & 0.1 & -- \\
\hline 2 & 5 & $10 / 4 / 1994$ & $<0.1$ & $<0.02$ & 0.12 & -- & -- & -- & $<0.02$ & -- & -- & -- & -- & -- & 0.1 & -- \\
\hline 2 & 6 & $7 / 12 / 1995$ & $<0.1$ & $<0.02$ & $<0.02$ & -- & -- & -- & $<0.02$ & -- & -- & -- & -- & -- & $<0.2$ & -- \\
\hline 2 & 7 & 9/27/1995 & $<0.1$ & $<0.02$ & $<0.02$ & -- & -- & -- & $<0.02$ & -- & -- & -- & -- & -- & $<0.2$ & -- \\
\hline 2 & 8 & 9/28/1995 & $<0.1$ & $<0.02$ & $<0.02$ & -- & -- & -- & $<0.02$ & -- & -- & -- & -- & -- & $<0.2$ & -- \\
\hline 2 & 9 & $12 / 14 / 1995$ & $<0.1$ & $<0.02$ & $<0.02$ & -- & -- & -- & $<0.02$ & -- & -- & -- & -- & -- & $<0.2$ & -- \\
\hline 2 & 10 & $12 / 15 / 1995$ & $<0.1$ & $<0.02$ & $<0.02$ & -- & -- & -- & $<0.02$ & -- & -- & -- & -- & -- & $<0.2$ & -- \\
\hline 2 & 11 & $3 / 8 / 1996$ & $<0.1$ & $<0.02$ & $<0.02$ & -- & -- & -- & $<0.02$ & -- & -- & -- & -- & -- & $<0.2$ & -- \\
\hline 2 & 12 & $3 / 11 / 1996$ & $<0.002$ & 0.002 & $<0.005$ & -- & -- & -- & 0.001 & $<0.2$ & 3.68 & 6.50 & 0.340 & $<0.2$ & $<0.3$ & 0.260 \\
\hline 2 & 13 & $6 / 10 / 1996$ & $<0.002$ & $<0.001$ & $<0.005$ & -- & -- & -- & 0.001 & $<0.2$ & 0.580 & 5.63 & 0.580 & $<0.2$ & $<0.3$ & $<0.200$ \\
\hline 2 & 14 & $6 / 12 / 1996$ & $<0.1$ & $<0.02$ & $<0.02$ & -- & -- & -- & $<0.02$ & -- & -- & -- & -- & -- & $<0.2$ & -- \\
\hline 2 & 15 & $6 / 14 / 1996$ & $<0.1$ & $<0.02$ & 0.02 & -- & -- & -- & $<0.02$ & -- & -- & -- & -- & -- & $<0.2$ & -- \\
\hline 2 & 16 & $6 / 14 / 1996$ & $<0.1$ & $<0.02$ & 0.03 & -- & -- & -- & $<0.02$ & -- & -- & -- & -- & -- & $<0.2$ & -- \\
\hline 2 & 17 & $8 / 21 / 1996$ & $<0.1$ & $<0.02$ & $<0.02$ & -- & -- & -- & $<0.02$ & -- & -- & -- & -- & -- & $<0.2$ & -- \\
\hline 2 & 18 & $8 / 22 / 1996$ & $<0.1$ & $<0.02$ & $<0.02$ & -- & -- & -- & $<0.02$ & -- & -- & -- & -- & -- & $<0.2$ & -- \\
\hline 2 & 19 & $8 / 23 / 1996$ & $<0.002$ & $<0.001$ & $<0.005$ & $<0.010$ & -- & -- & $<0.001$ & $<0.2$ & $<0.300$ & $<2$ & $<0.200$ & $<0.2$ & $<0.3$ & $<0.200$ \\
\hline 2 & 20 & $8 / 23 / 1996$ & $<0.002$ & $<0.001$ & $<0.005$ & 0.010 & -- & -- & $<0.002$ & $<0.2$ & $<0.300$ & $<2$ & $<0.200$ & $<0.2$ & $<0.3$ & $<0.200$ \\
\hline 2 & 21 & $8 / 28 / 1996$ & $<0.1$ & $<0.02$ & $<0.02$ & -- & -- & -- & $<0.02$ & -- & -- & -- & -- & -- & $<0.2$ & -- \\
\hline 2 & 22 & 8/29/1996 & $<0.002$ & $<0.001$ & $<0.005$ & $<0.010$ & -- & -- & $<0.001$ & $<0.2$ & 0.360 & 4.67 & $<0.200$ & $<0.2$ & $<0.3$ & $<0.200$ \\
\hline 2 & 23 & $8 / 29 / 1996$ & $<0.002$ & $<0.001$ & $<0.005$ & -- & -- & -- & $<0.001$ & $<0.2$ & $<0.300$ & $<2.00$ & $<0.200$ & $<0.2$ & $<0.3$ & $<0.200$ \\
\hline 2 & 24 & $11 / 8 / 1996$ & $<0.1$ & $<0.02$ & 0.05 & -- & -- & -- & $<0.02$ & -- & -- & -- & -- & -- & $<0.2$ & -- \\
\hline 2 & 25 & $11 / 8 / 1996$ & $<0.1$ & $<0.02$ & $<0.02$ & -- & -- & -- & $<0.02$ & -- & -- & -- & -- & -- & $<0.2$ & -- \\
\hline 2 & 26 & $2 / 25 / 1997$ & $<0.1$ & $<0.02$ & $<0.02$ & -- & -- & -- & $<0.02$ & -- & -- & -- & -- & -- & $<0.2$ & -- \\
\hline 2 & 27 & $2 / 26 / 1997$ & $<0.1$ & 0.02 & $<0.02$ & -- & -- & -- & 0.04 & -- & -- & -- & -- & -- & $<0.2$ & -- \\
\hline 2 & 28 & $6 / 4 / 1997$ & $<0.1$ & $<0.02$ & $<0.02$ & -- & -- & -- & $<0.02$ & -- & -- & -- & -- & -- & $<0.2$ & -- \\
\hline 2 & 29 & $6 / 5 / 1997$ & 0.02 & -- & $<0.050$ & $<0.01$ & 0.01 & -- & $<0.01$ & $<0.20$ & 0.66 & 4.43 & $<0.20$ & $<0.20$ & $<0.30$ & $<0.20$ \\
\hline 2 & 30 & $8 / 27 / 1997$ & $<0.1$ & $<0.02$ & 0.02 & -- & -- & -- & $<0.02$ & -- & -- & -- & -- & -- & $<0.2$ & -- \\
\hline 2 & 31 & $8 / 26 / 1997$ & $<0.1$ & $<0.02$ & $<0.02$ & -- & -- & -- & $<0.02$ & -- & -- & -- & -- & -- & $<0.2$ & -- \\
\hline 2 & 32 & $8 / 29 / 1997$ & $<0.1$ & $<0.02$ & $<0.02$ & -- & -- & -- & $<0.02$ & -- & -- & -- & -- & -- & $<0.2$ & -- \\
\hline 2 & 33 & $11 / 19 / 1997$ & $<0.1$ & $<0.02$ & $<0.02$ & -- & -- & -- & $<0.02$ & -- & -- & -- & -- & -- & $<0.2$ & -- \\
\hline 2 & 34 & $11 / 20 / 1997$ & $<0.1$ & $<0.02$ & $<0.02$ & -- & -- & -- & $<0.02$ & -- & -- & -- & -- & -- & $<0.2$ & -- \\
\hline 2 & 35 & $11 / 21 / 1997$ & $<0.02$ & 0.001 & $<0.050$ & $<0.01$ & $<0.01$ & -- & 0.001 & $<0.20$ & $<0.30$ & $<2$ & $<0.20$ & $<0.20$ & $<0.30$ & 0.38 \\
\hline 2 & 36 & $12 / 4 / 1997$ & $<0.02$ & 0.001 & $<0.050$ & $<0.01$ & $<0.01$ & -- & $<0.001$ & $<0.20$ & $<0.30$ & 3.08 & $<0.20$ & $<0.20$ & $<0.30$ & $<0.20$ \\
\hline 2 & 37 & $1 / 8 / 1998$ & $<0.1$ & $<0.02$ & 0.04 & -- & -- & -- & $<0.02$ & -- & -- & -- & -- & -- & $<0.2$ & -- \\
\hline 2 & 38 & $1 / 8 / 1998$ & $<0.1$ & $<0.02$ & $<0.02$ & -- & -- & -- & $<0.02$ & -- & -- & -- & -- & -- & 0.1 & -- \\
\hline 2 & 39 & $1 / 15 / 1998$ & $<0.02$ & 0.001 & $<0.050$ & $<0.01$ & $<0.010$ & -- & $<0.001$ & $<0.20$ & $<0.30$ & $<2$ & $<0.20$ & $<0.20$ & $<0.30$ & $<0.20$ \\
\hline 2 & 40 & $3 / 27 / 1998$ & $<0.002$ & $<0.001$ & $<0.005$ & $<0.01$ & 0.02 & -- & $<0.01$ & $<0.20$ & $<0.30$ & $<2$ & $<0.20$ & $<0.20$ & $<0.30$ & $<0.20$ \\
\hline 2 & 41 & 4/16/1998 & $<0.1$ & $<0.02$ & $<0.02$ & -- & -- & -- & $<0.02$ & -- & -- & -- & -- & -- & $<0.2$ & -- \\
\hline 2 & 42 & $4 / 16 / 1998$ & $<0.1$ & $<0.02$ & $<0.02$ & -- & -- & -- & $<0.02$ & -- & -- & -- & -- & -- & $<0.2$ & -- \\
\hline 2 & 43 & $7 / 15 / 1998$ & $<0.1$ & $<0.02$ & $<0.02$ & -- & -- & -- & $<0.02$ & -- & -- & -- & -- & -- & $<0.2$ & -- \\
\hline 2 & 44 & $7 / 16 / 1998$ & $<0.1$ & $<0.02$ & $<0.02$ & -- & -- & -- & $<0.02$ & -- & -- & -- & -- & -- & $<0.2$ & -- \\
\hline 2 & 45 & $7 / 17 / 1998$ & $<0.002$ & $<0.001$ & $<0.005$ & 0.01 & $<0.01$ & -- & $<0.001$ & $<0.20$ & $<0.30$ & $<2$ & $<0.20$ & $<0.20$ & $<0.30$ & $<0.20$ \\
\hline 2 & 46 & $7 / 21 / 1998$ & $<0.02$ & -- & $<0.050$ & 0.01 & $<0.01$ & -- & $<0.01$ & $<0.20$ & $<0.30$ & 2.49 & $<0.20$ & $<0.20$ & $<0.30$ & $<0.20$ \\
\hline 2 & 47 & $7 / 21 / 1998$ & 0.02 & -- & $<0.050$ & 0.01 & $<0.01$ & -- & $<0.01$ & $<0.20$ & $<0.30$ & $<2$ & $<0.20$ & $<0.20$ & $<0.30$ & $<0.20$ \\
\hline 2 & 48 & 7/13/1999 & $<0.02$ & -- & $<0.050$ & -- & $<0.05$ & $<0.05$ & -- & $<1$ & $<1$ & $<16$ & $<1$ & $<1$ & $<1$ & $<1$ \\
\hline 3 & 49 & $4 / 22 / 1994$ & $<0.1$ & $<0.02$ & 0.03 & -- & -- & -- & $<0.02$ & -- & -- & -- & -- & -- & $<0.2$ & -- \\
\hline
\end{tabular}


Appendix 7. Information and chemical data for blank water-quality samples collected near Deer Trail, Colorado, 1993 through 1999.—Continued

[Codes are explained in appendix 6; other abbreviations are listed in appendix 2; mg/L, milligrams per liter; $\mu \mathrm{S} / \mathrm{cm}$, microsiemens per centimeter at 25 degrees Celsius; $\mu \mathrm{g} / \mathrm{L}$, micrograms per liter; all data from filtered samples unless otherwise indicated; $<$, less than; --, no data]

\begin{tabular}{|c|c|c|c|c|c|c|c|c|c|c|c|c|c|c|}
\hline $\begin{array}{c}\text { Sampling } \\
\text { type }\end{array}$ & $\begin{array}{c}\text { Blank } \\
\text { number }\end{array}$ & $\begin{array}{c}\text { Sample } \\
\text { date }\end{array}$ & $\begin{array}{c}\mathrm{Cr}, \\
\mu \mathrm{g} / \mathrm{L}\end{array}$ & $\begin{array}{c}\mathrm{Cu}, \\
\mu \mathrm{g} / \mathrm{L}\end{array}$ & $\begin{array}{c}\mathrm{Fe}, \\
\mu \mathrm{g} / \mathrm{L}\end{array}$ & $\begin{array}{c}\mathrm{Mn}, \\
\mu \mathrm{g} / \mathrm{L}\end{array}$ & $\begin{array}{l}\text { Mo, } \\
\mu \mathrm{gg} / \mathrm{L}\end{array}$ & $\begin{array}{c}\mathrm{Ni}, \\
\mu \mathrm{g} / \mathrm{L}\end{array}$ & $\begin{array}{c}\mathrm{Pb}, \\
\mu \mathrm{g} / \mathrm{L}\end{array}$ & $\begin{array}{l}\mathrm{Sb}, \\
\mu \mathrm{g} / \mathrm{L}\end{array}$ & $\begin{array}{c}\mathrm{Sr}, \\
\mu \mathrm{g} / \mathrm{L}\end{array}$ & $\begin{array}{c}\mathrm{Tl}, \\
\mu \mathrm{g} / \mathrm{L}\end{array}$ & $\begin{array}{c}U, \\
\mu g / L\end{array}$ & $\begin{array}{c}\mathrm{Zn}, \\
\mu \mathrm{g} / \mathrm{L}\end{array}$ \\
\hline 2 & 1 & $7 / 20 / 1994$ & $<100$ & $<100$ & $<250$ & $<100$ & -- & $<100$ & $<10$ & - & -- & -- & -- & $<100$ \\
\hline 2 & 2 & $10 / 3 / 1994$ & $<40$ & $<40$ & $<100$ & $<40$ & -- & $<40$ & $<10$ & -- & -- & -- & -- & $<40$ \\
\hline 2 & 3 & $10 / 3 / 1994$ & $<20$ & $<20$ & $<50$ & $<20$ & -- & $<20$ & $<10$ & -- & -- & -- & -- & $<20$ \\
\hline 2 & 4 & $10 / 4 / 1994$ & $<20$ & $<20$ & $<50$ & $<20$ & -- & $<20$ & $<10$ & -- & -- & -- & -- & $<20$ \\
\hline 2 & 5 & $10 / 4 / 1994$ & $<20$ & $<20$ & $<50$ & $<20$ & -- & $<20$ & $<10$ & -- & -- & -- & -- & $<20$ \\
\hline 2 & 6 & $7 / 12 / 1995$ & $<20$ & $<20$ & $<50$ & $<20$ & -- & $<20$ & $<10$ & -- & -- & -- & -- & $<20$ \\
\hline 2 & 7 & $9 / 27 / 1995$ & $<20$ & $<20$ & $<50$ & $<20$ & -- & 30 & $<10$ & -- & -- & -- & -- & $<20$ \\
\hline 2 & 8 & 9/28/1995 & $<20$ & $<20$ & $<50$ & $<20$ & -- & $<20$ & $<10$ & -- & -- & -- & -- & $<20$ \\
\hline 2 & 9 & $12 / 14 / 1995$ & $<20$ & $<20$ & $<50$ & $<20$ & -- & $<20$ & $<10$ & -- & -- & -- & -- & $<20$ \\
\hline 2 & 10 & $12 / 15 / 1995$ & $<20$ & $<20$ & $<50$ & $<20$ & -- & $<20$ & $<10$ & -- & -- & -- & -- & $<20$ \\
\hline 2 & 11 & $3 / 8 / 1996$ & $<20$ & $<20$ & $<50$ & $<20$ & -- & $<20$ & $<10$ & -- & -- & -- & -- & $<20$ \\
\hline 2 & 12 & $3 / 11 / 1996$ & $<0.200$ & 0.570 & $<3$ & 0.770 & 1 & 0.820 & $<0.3$ & $<0.200$ & 0.2 & $<0.100$ & $<0.20$ & 7 \\
\hline 2 & 13 & $6 / 10 / 1996$ & $<0.200$ & 3 & $<3$ & 2 & $<0.200$ & 1 & $<0.300$ & $<0.200$ & 0.520 & $<0.100$ & $<0.20$ & 3 \\
\hline 2 & 14 & $6 / 12 / 1996$ & $<20$ & $<20$ & $<50$ & $<20$ & -- & $<20$ & $<10$ & -- & -- & -- & -- & $<20$ \\
\hline 2 & 15 & $6 / 14 / 1996$ & $<20$ & $<20$ & $<50$ & $<20$ & -- & $<20$ & $<10$ & -- & -- & -- & -- & $<20$ \\
\hline 2 & 16 & $6 / 14 / 1996$ & $<20$ & $<20$ & $<50$ & $<20$ & -- & $<20$ & $<10$ & -- & -- & -- & -- & $<20$ \\
\hline 2 & 17 & $8 / 21 / 1996$ & $<20$ & $<20$ & $<50$ & $<20$ & -- & $<20$ & $<10$ & -- & -- & -- & -- & $<20$ \\
\hline 2 & 18 & $8 / 22 / 1996$ & $<20$ & $<20$ & $<50$ & $<20$ & -- & $<20$ & $<10$ & -- & -- & -- & -- & $<20$ \\
\hline 2 & 19 & $8 / 23 / 1996$ & $<0.200$ & $<0.200$ & $<3$ & $<0.100$ & $<0.200$ & $<0.500$ & $<0.300$ & $<0.200$ & $<0.100$ & $<0.100$ & $<0.20$ & $<0.500$ \\
\hline 2 & 20 & $8 / 23 / 1996$ & $<0.200$ & $<0.200$ & $<3$ & $<0.100$ & $<0.200$ & $<0.500$ & $<0.300$ & $<0.200$ & $<0.100$ & $<0.100$ & $<0.20$ & $<0.500$ \\
\hline 2 & 21 & $8 / 28 / 1996$ & $<20$ & $<20$ & $<50$ & $<20$ & -- & $<20$ & $<10$ & -- & -- & -- & -- & $<20$ \\
\hline 2 & 22 & $8 / 29 / 1996$ & $<0.200$ & 2 & 3 & 0.460 & $<0.200$ & $<0.500$ & $<0.300$ & $<0.200$ & 0.150 & $<0.100$ & $<0.20$ & 2 \\
\hline 2 & 23 & $8 / 29 / 1996$ & $<0.200$ & $<0.200$ & $<3$ & $<0.100$ & $<0.200$ & $<0.500$ & $<0.300$ & $<0.200$ & $<0.100$ & $<0.100$ & $<0.20$ & $<0.500$ \\
\hline 2 & 24 & $11 / 8 / 1996$ & $<20$ & $<20$ & $<50$ & $<20$ & -- & $<20$ & $<10$ & -- & -- & -- & -- & $<20$ \\
\hline 2 & 25 & $11 / 8 / 1996$ & $<20$ & $<20$ & $<50$ & $<20$ & -- & $<20$ & $<10$ & -- & -- & -- & -- & $<20$ \\
\hline 2 & 26 & $2 / 25 / 1997$ & $<20$ & $<20$ & $<50$ & $<20$ & -- & $<20$ & $<10$ & -- & -- & -- & -- & $<20$ \\
\hline 2 & 27 & 2/26/1997 & $<20$ & $<20$ & $<50$ & $<20$ & -- & $<20$ & $<10$ & -- & -- & -- & -- & $<20$ \\
\hline 2 & 28 & $6 / 4 / 1997$ & $<20$ & $<20$ & 50 & $<20$ & -- & $<20$ & $<10$ & -- & -- & -- & -- & $<20$ \\
\hline 2 & 29 & 6/5/1997 & $<0.20$ & 1.50 & $<3$ & 0.56 & $<0.20$ & 0.53 & $<0.30$ & $<0.20$ & 0.24 & $<0.10$ & $<0.20$ & 1.36 \\
\hline 2 & 30 & $8 / 27 / 1997$ & $<20$ & $<20$ & $<50$ & $<20$ & -- & $<20$ & $<10$ & -- & -- & -- & -- & $<20$ \\
\hline 2 & 31 & 8/26/1997 & $<20$ & $<20$ & $<50$ & $<20$ & -- & $<20$ & $<10$ & -- & -- & -- & -- & $<20$ \\
\hline 2 & 32 & 8/29/1997 & $<20$ & $<20$ & $<50$ & $<20$ & -- & $<20$ & $<10$ & -- & -- & -- & -- & $<20$ \\
\hline 2 & 33 & $11 / 19 / 1997$ & $<20$ & $<20$ & $<50$ & $<20$ & -- & $<20$ & $<10$ & -- & -- & -- & -- & $<20$ \\
\hline 2 & 34 & $11 / 20 / 1997$ & $<20$ & $<20$ & $<50$ & $<20$ & -- & $<20$ & $<10$ & -- & -- & -- & -- & $<20$ \\
\hline 2 & 35 & $11 / 21 / 1997$ & $<0.20$ & 0.55 & $<3$ & 0.24 & $<0.20$ & $<0.50$ & $<0.30$ & $<0.20$ & 0.24 & $<0.10$ & $<0.20$ & $<0.50$ \\
\hline 2 & 36 & 12/4/1997 & $<0.20$ & $<0.20$ & $<3$ & $<0.10$ & $<0.20$ & $<0.50$ & $<0.30$ & $<0.20$ & $<0.10$ & $<0.10$ & $<0.20$ & $<0.50$ \\
\hline 2 & 37 & $1 / 8 / 1998$ & $<20$ & $<20$ & $<50$ & $<20$ & -- & $<20$ & $<10$ & -- & -- & -- & -- & $<20$ \\
\hline 2 & 38 & $1 / 8 / 1998$ & $<20$ & $<20$ & $<50$ & $<20$ & -- & $<20$ & $<10$ & -- & -- & -- & -- & $<20$ \\
\hline 2 & 39 & $1 / 15 / 1998$ & $<0.20$ & 0.91 & $<3$ & $<0.10$ & $<0.20$ & $<0.50$ & $<0.30$ & $<0.20$ & 0.10 & $<0.10$ & $<0.20$ & 1.13 \\
\hline 2 & 40 & $3 / 27 / 1998$ & $<0.20$ & 0.40 & $<3$ & 0.22 & $<0.20$ & $<0.50$ & $<0.30$ & $<0.20$ & $<0.10$ & $<0.10$ & $<0.20$ & $<0.50$ \\
\hline 2 & 41 & $4 / 16 / 1998$ & $<20$ & $<20$ & $<50$ & $<20$ & -- & $<20$ & $<10$ & -- & -- & -- & -- & $<20$ \\
\hline 2 & 42 & $4 / 16 / 1998$ & $<20$ & $<20$ & $<50$ & $<20$ & -- & $<20$ & $<10$ & -- & -- & -- & -- & $<20$ \\
\hline 2 & 43 & $7 / 15 / 1998$ & $<20$ & $<20$ & $<50$ & $<20$ & -- & $<20$ & $<10$ & -- & -- & -- & -- & $<20$ \\
\hline 2 & 44 & $7 / 16 / 1998$ & $<20$ & $<20$ & $<50$ & $<20$ & -- & $<20$ & $<10$ & -- & -- & -- & -- & $<20$ \\
\hline 2 & 45 & $7 / 17 / 1998$ & $<0.2$ & $<0.20$ & $<3$ & $<0.10$ & $<0.20$ & $<0.50$ & $<0.30$ & $<0.20$ & $<0.10$ & $<0.10$ & $<0.20$ & $<0.50$ \\
\hline 2 & 46 & $7 / 21 / 1998$ & $<0.20$ & $<0.20$ & $<3$ & $<0.10$ & $<0.20$ & $<0.50$ & $<0.30$ & $<0.20$ & $<0.10$ & $<0.10$ & $<0.20$ & $<0.50$ \\
\hline 2 & 47 & $7 / 21 / 1998$ & $<0.20$ & $<0.20$ & $<3$ & $<0.10$ & $<0.20$ & $<0.50$ & $<0.30$ & $<0.20$ & $<0.10$ & $<0.10$ & $<0.20$ & $<0.50$ \\
\hline 2 & 48 & $7 / 13 / 1999$ & $<1.0$ & $<1$ & $<10$ & $<1$ & $<1$ & $<1$ & $<1$ & $<1$ & $<1$ & -- & $<1$ & $<1.0$ \\
\hline 3 & 49 & $4 / 22 / 1994$ & $<20$ & 40 & $<50$ & $<20$ & -- & $<20$ & $<10$ & -- & -- & -- & -- & $<20$ \\
\hline
\end{tabular}


Appendix 8. Performance-evaluation data from the U.S. Geological Survey Standard Reference Program for the Metro Wastewater Reclamation District Laboratory (1993-1998) and the U.S. Geological Survey National Water Quality Laboratory (1993-1999).

[All abbreviations explained in appendix 2; USGS, U.S. Geological Survey; Metro District, Metro Wastewater Reclamation District Laboratory; NWQL, National Water Quality Laboratory; $\mu \mathrm{g} / \mathrm{L}$, micrograms per liter; $\mathrm{mg} / \mathrm{L}$, milligrams per liter; $\mu \mathrm{S} / \mathrm{cm}$, microsiemens per centimeter at 25 degrees Celsius; ICP, inductively coupled plasma; MS, mass spectrometry; --; no data; na, not applicable; AA, atomic absorption spectrometry; GFAA, graphite-furnace atomic absorption spectrometry; <, less than; DCP, direct current plasma; IC, ion chromatography]

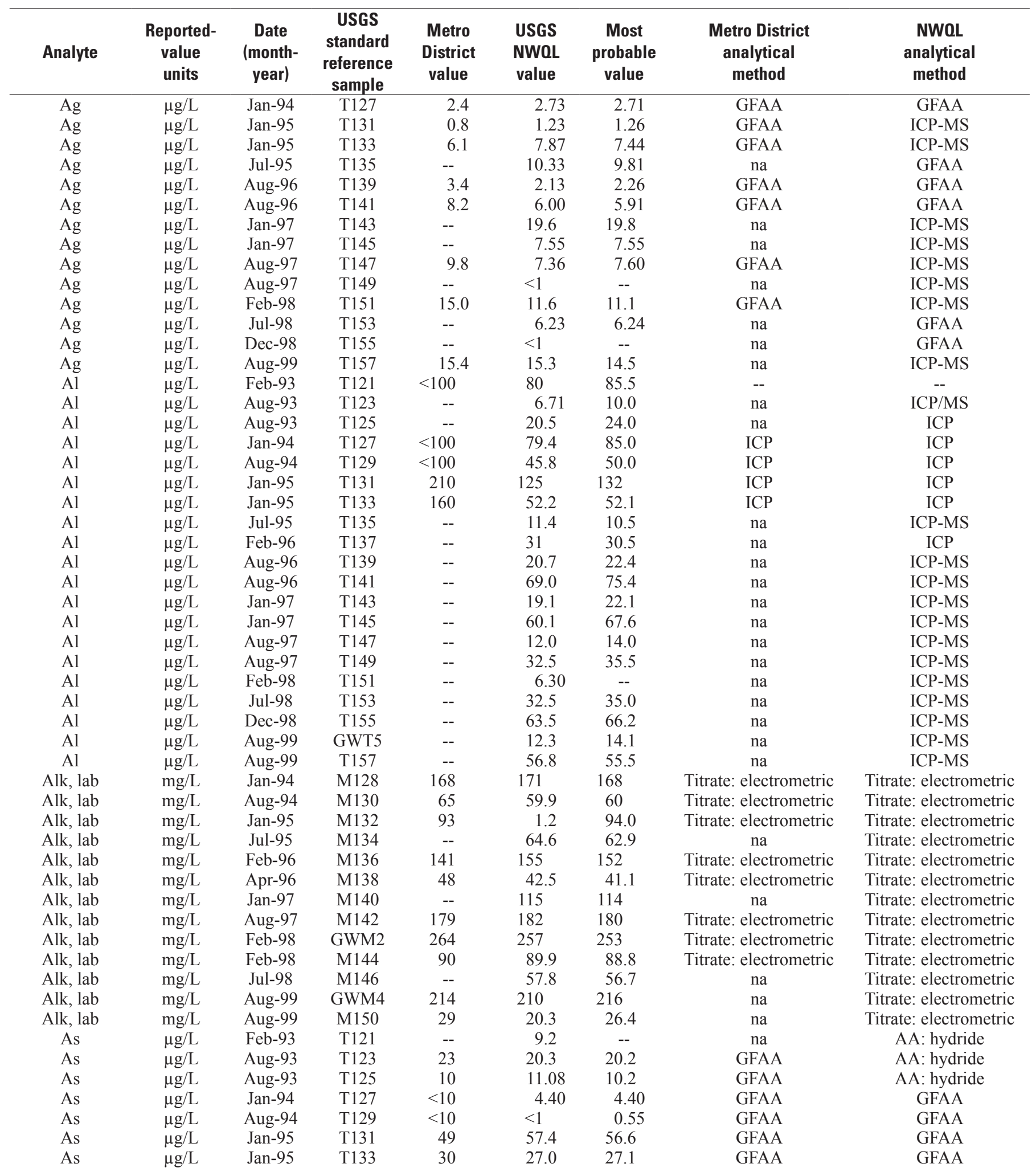


Appendix 8. Performance-evaluation data from the U.S. Geological Survey Standard Reference Program for the Metro Wastewater Reclamation District Laboratory (1993-1998) and the U.S. Geological Survey National Water Quality Laboratory (1993-1999).—Continued

[All abbreviations explained in appendix 2; USGS, U.S. Geological Survey; Metro District, Metro Wastewater Reclamation District Laboratory; NWQL, National Water Quality Laboratory; $\mu \mathrm{g} / \mathrm{L}$, micrograms per liter; $\mathrm{mg} / \mathrm{L}$, milligrams per liter; $\mu \mathrm{S} / \mathrm{cm}$, microsiemens per centimeter at 25 degrees Celsius; ICP, inductively coupled plasma; MS, mass spectrometry; --; no data; na, not applicable; AA, atomic absorption spectrometry; GFAA, graphite-furnace atomic absorption spectrometry; <, less than; DCP, direct current plasma; IC, ion chromatography]

\begin{tabular}{|c|c|c|c|c|c|c|c|c|}
\hline Analyte & $\begin{array}{l}\text { Reported- } \\
\text { value } \\
\text { units }\end{array}$ & $\begin{array}{c}\text { Date } \\
\text { (month- } \\
\text { year) }\end{array}$ & $\begin{array}{c}\text { USGS } \\
\text { standard } \\
\text { reference } \\
\text { sample }\end{array}$ & $\begin{array}{c}\text { Metro } \\
\text { District } \\
\text { value }\end{array}$ & $\begin{array}{l}\text { USGS } \\
\text { NWOL } \\
\text { value }\end{array}$ & $\begin{array}{c}\text { Most } \\
\text { probable } \\
\text { value }\end{array}$ & $\begin{array}{l}\text { Metro District } \\
\text { analytical } \\
\text { method }\end{array}$ & $\begin{array}{l}\text { NWOL } \\
\text { analytical } \\
\text { method }\end{array}$ \\
\hline As & $\mu \mathrm{g} / \mathrm{L}$ & Jul-95 & $\mathrm{T} 135$ & -- & 10.0 & 10.0 & na & GFAA \\
\hline As & $\mu \mathrm{g} / \mathrm{L}$ & Feb-96 & $\mathrm{T} 137$ & -- & $<1$ & 0.6 & na & GFAA \\
\hline As & $\mu \mathrm{g} / \mathrm{L}$ & Aug-96 & T139 & -- & 5.47 & 5.55 & na & GFAA \\
\hline As & $\mu \mathrm{g} / \mathrm{L}$ & Aug-96 & $\mathrm{T} 141$ & 10 & 7.50 & 7.50 & GFAA & GFAA \\
\hline As & $\mu \mathrm{g} / \mathrm{L}$ & Jan-97 & $\mathrm{T} 143$ & -- & 16.2 & 15.2 & na & GFAA \\
\hline As & $\mu \mathrm{g} / \mathrm{L}$ & Jan-97 & $\mathrm{T} 145$ & -- & 9.90 & 9.88 & na & GFAA \\
\hline As & $\mu \mathrm{g} / \mathrm{L}$ & Aug-97 & $\mathrm{T} 147$ & -- & 2.20 & 2.39 & na & GFAA \\
\hline As & $\mu \mathrm{g} / \mathrm{L}$ & Aug-97 & T149 & -- & $<1$ & 1.0 & na & GFAA \\
\hline As & $\mu \mathrm{g} / \mathrm{L}$ & Feb-98 & $\mathrm{T} 151$ & -- & 1.60 & 1.01 & na & GFAA \\
\hline As & $\mu \mathrm{g} / \mathrm{L}$ & Jul-98 & T153 & -- & $<1$ & 0.50 & na & GFAA \\
\hline As & $\mu \mathrm{g} / \mathrm{L}$ & Dec-98 & $\mathrm{T} 155$ & 35 & 31.9 & 32.9 & GFAA & GFAA \\
\hline As & $\mu \mathrm{g} / \mathrm{L}$ & Aug-99 & GWT5 & 22.0 & 22.8 & 21.9 & GFAA & GFAA \\
\hline As & $\mu \mathrm{g} / \mathrm{L}$ & Aug-99 & T157 & 23 & 27.6 & 25.4 & GFAA & GFAA \\
\hline $\mathrm{B}$ & $\mu \mathrm{g} / \mathrm{L}$ & Feb-93 & $\mathrm{T} 121$ & - & 93 & 90 & na & DCP \\
\hline B & $\mu \mathrm{g} / \mathrm{L}$ & Aug-93 & M126 & -- & 8.44 & 9.6 & na & $\mathrm{ICP}$ \\
\hline B & $\mu \mathrm{g} / \mathrm{L}$ & Aug-93 & $\mathrm{T} 123$ & -- & 9.75 & 11.3 & na & ICP \\
\hline B & $\mu \mathrm{g} / \mathrm{L}$ & Aug-93 & $\mathrm{T} 125$ & -- & 19.21 & 19.4 & na & ICP \\
\hline B & $\mu \mathrm{g} / \mathrm{L}$ & Jan-94 & M128 & -- & 297 & 285 & na & ICP \\
\hline B & $\mu \mathrm{g} / \mathrm{L}$ & Jan-94 & T127 & -- & 42.7 & 42.8 & na & ICP \\
\hline B & $\mu \mathrm{g} / \mathrm{L}$ & Aug-94 & M130 & -- & 7.66 & 8.87 & na & ICP \\
\hline B & $\mu \mathrm{g} / \mathrm{L}$ & Aug-94 & T129 & -- & 8.9 & 11.6 & na & ICP \\
\hline B & $\mu \mathrm{g} / \mathrm{L}$ & Jan-95 & M132 & -- & 30.7 & 30.4 & na & ICP \\
\hline B & $\mu \mathrm{g} / \mathrm{L}$ & Jan-95 & $\mathrm{T} 131$ & -- & 143 & 141 & na & ICP \\
\hline B & $\mu \mathrm{g} / \mathrm{L}$ & Jan-95 & $\mathrm{T} 133$ & -- & 300 & 297 & na & $\mathrm{ICP}$ \\
\hline B & $\mu \mathrm{g} / \mathrm{L}$ & Jul-95 & M134 & -- & 35.1 & 33.7 & na & ICP \\
\hline B & $\mu \mathrm{g} / \mathrm{L}$ & Jul-95 & $\mathrm{T} 135$ & -- & 13.3 & 13.1 & na & ICP \\
\hline B & $\mu \mathrm{g} / \mathrm{L}$ & Feb-96 & M136 & -- & 202 & 200 & na & DCP \\
\hline B & $\mu \mathrm{g} / \mathrm{L}$ & Feb-96 & T137 & -- & $<10$ & 15.6 & na & DCP \\
\hline B & $\mu \mathrm{g} / \mathrm{L}$ & Apr-96 & M138 & -- & 5 & 10 & na & ICP \\
\hline B & $\mu \mathrm{g} / \mathrm{L}$ & Aug-96 & T139 & -- & 36 & 36 & na & DCP \\
\hline B & $\mu \mathrm{g} / \mathrm{L}$ & Aug-96 & $\mathrm{T} 141$ & -- & 30 & 29 & na & $\mathrm{ICP}$ \\
\hline B & $\mu \mathrm{g} / \mathrm{L}$ & Jan-97 & M140 & -- & 43.6 & 41.6 & na & ICP \\
\hline B & $\mu \mathrm{g} / \mathrm{L}$ & Jan-97 & $\mathrm{T} 143$ & -- & 34.9 & 35.0 & na & $\mathrm{ICP}$ \\
\hline B & $\mu \mathrm{g} / \mathrm{L}$ & Jan-97 & $\mathrm{T} 145$ & -- & 45.7 & 45.6 & na & ICP \\
\hline B & $\mu \mathrm{g} / \mathrm{L}$ & Aug-97 & M142 & -- & 114 & 121 & na & ICP \\
\hline B & $\mu \mathrm{g} / \mathrm{L}$ & Aug-97 & $\mathrm{T} 147$ & -- & 50.4 & 50.0 & na & ICP \\
\hline B & $\mu \mathrm{g} / \mathrm{L}$ & Aug-97 & T149 & -- & 124 & 128 & na & ICP \\
\hline B & $\mu \mathrm{g} / \mathrm{L}$ & Feb-98 & GWM2 & -- & 235 & 238 & na & ICP \\
\hline B & $\mu \mathrm{g} / \mathrm{L}$ & Feb-98 & M144 & -- & 45.7 & 46.1 & na & ICP \\
\hline B & $\mu \mathrm{g} / \mathrm{L}$ & Feb-98 & $\mathrm{T} 151$ & -- & 36.4 & 36.3 & na & ICP \\
\hline B & $\mu \mathrm{g} / \mathrm{L}$ & Jul-98 & M146 & -- & 14.2 & 13.9 & na & ICP \\
\hline B & $\mu \mathrm{g} / \mathrm{L}$ & Jul-98 & $\mathrm{T} 153$ & -- & 97.1 & 99.4 & na & ICP \\
\hline B & $\mu \mathrm{g} / \mathrm{L}$ & Dec-98 & $\mathrm{T} 155$ & -- & 94.7 & 94.0 & na & ICP \\
\hline $\mathrm{B}$ & $\mu \mathrm{g} / \mathrm{L}$ & Aug-99 & GWM4 & -- & 95.2 & 95.2 & na & ICP \\
\hline $\mathrm{B}$ & $\mu \mathrm{g} / \mathrm{L}$ & Aug-99 & GWT5 & -- & 98.2 & 98.6 & na & ICP \\
\hline $\mathrm{B}$ & $\mu \mathrm{g} / \mathrm{L}$ & Aug-99 & $\mathrm{T} 157$ & -- & 70.8 & 70.4 & na & ICP \\
\hline $\mathrm{Ba}$ & $\mu \mathrm{g} / \mathrm{L}$ & Feb-93 & $\mathrm{T} 121$ & -- & 46.28 & 46.3 & na & ICP \\
\hline $\mathrm{Ba}$ & $\mu \mathrm{g} / \mathrm{L}$ & Aug-93 & $\mathrm{T} 123$ & -- & 7.25 & 7.65 & na & ICP \\
\hline $\mathrm{Ba}$ & $\mu \mathrm{g} / \mathrm{L}$ & Aug-93 & $\mathrm{T} 125$ & -- & 16.3 & 16.9 & na & ICP \\
\hline $\mathrm{Ba}$ & $\mu \mathrm{g} / \mathrm{L}$ & Jan-94 & $\mathrm{T} 127$ & -- & 19.7 & 20.6 & na & ICP \\
\hline $\mathrm{Ba}$ & $\mu \mathrm{g} / \mathrm{L}$ & Aug-94 & T129 & -- & 33.0 & 34.0 & na & ICP \\
\hline $\mathrm{Ba}$ & $\mu \mathrm{g} / \mathrm{L}$ & Jan-95 & $\mathrm{T} 131$ & -- & 495 & 507 & na & ICP-MS \\
\hline $\mathrm{Ba}$ & $\mu \mathrm{g} / \mathrm{L}$ & Jan-95 & $\mathrm{T} 133$ & -- & 151 & 148 & na & ICP-MS \\
\hline $\mathrm{Ba}$ & $\mu \mathrm{g} / \mathrm{L}$ & Jul-95 & $\mathrm{T} 135$ & -- & 70.6 & 67.8 & na & ICP \\
\hline
\end{tabular}


Appendix 8. Performance-evaluation data from the U.S. Geological Survey Standard Reference Program for the Metro Wastewater Reclamation District Laboratory (1993-1998) and the U.S. Geological Survey National Water Quality Laboratory (1993-1999).—Continued

[All abbreviations explained in appendix 2; USGS, U.S. Geological Survey; Metro District, Metro Wastewater Reclamation District Laboratory; NWQL, National Water Quality Laboratory; $\mu \mathrm{g} / \mathrm{L}$, micrograms per liter; $\mathrm{mg} / \mathrm{L}$, milligrams per liter; $\mu \mathrm{S} / \mathrm{cm}$, microsiemens per centimeter at 25 degrees Celsius; ICP, inductively coupled plasma; MS, mass spectrometry; --; no data; na, not applicable; AA, atomic absorption spectrometry; GFAA, graphite-furnace atomic absorption spectrometry; <, less than; DCP, direct current plasma; IC, ion chromatography]

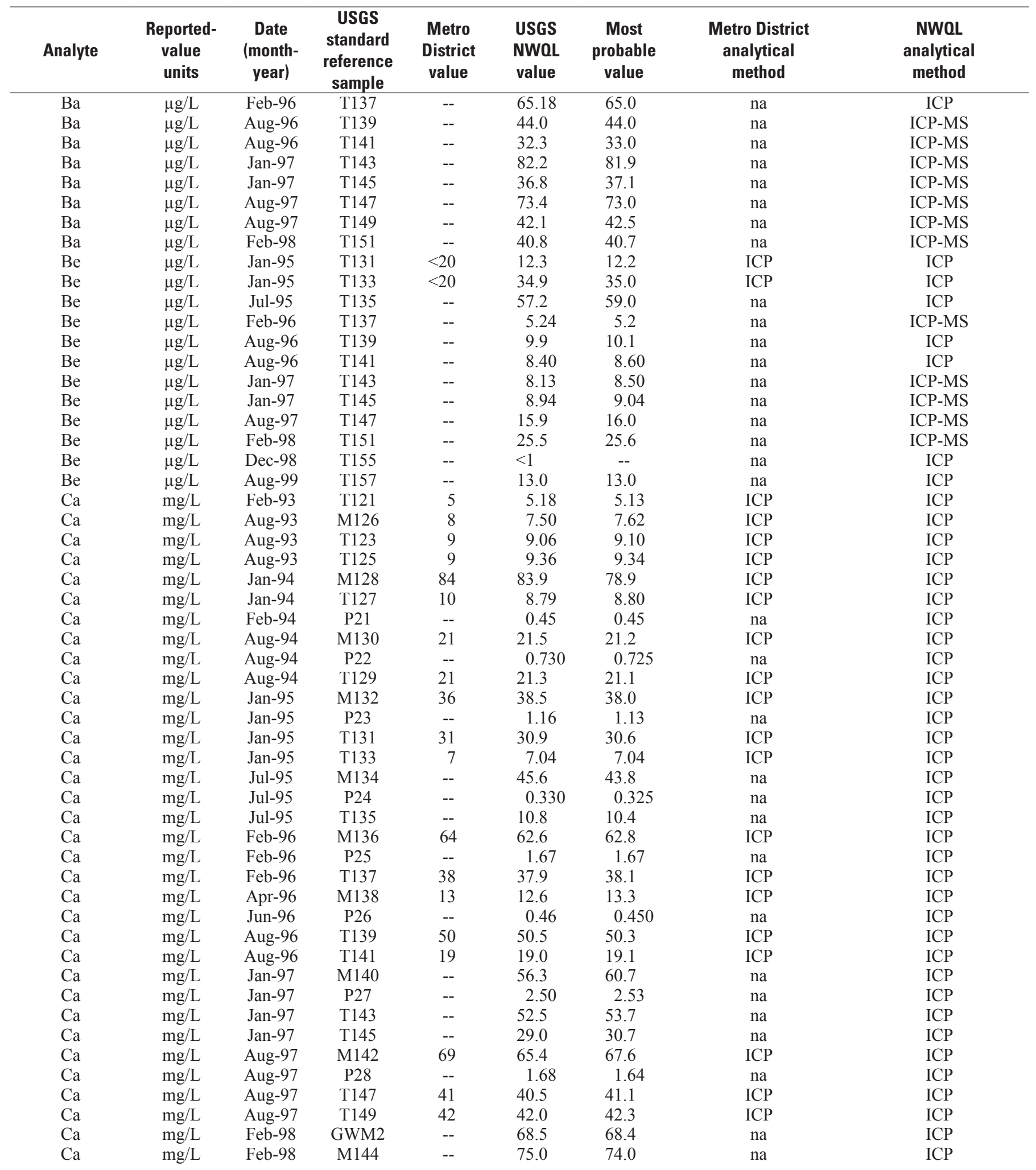


Appendix 8. Performance-evaluation data from the U.S. Geological Survey Standard Reference Program for the Metro Wastewater Reclamation District Laboratory (1993-1998) and the U.S. Geological Survey National Water Quality Laboratory (1993-1999).-Continued

[All abbreviations explained in appendix 2; USGS, U.S. Geological Survey; Metro District, Metro Wastewater Reclamation District Laboratory; NWQL, National Water Quality Laboratory; $\mu \mathrm{g} / \mathrm{L}$, micrograms per liter; $\mathrm{mg} / \mathrm{L}$, milligrams per liter; $\mu \mathrm{S} / \mathrm{cm}$, microsiemens per centimeter at 25 degrees Celsius; ICP, inductively coupled plasma; MS, mass spectrometry; --; no data; na, not applicable; AA, atomic absorption spectrometry; GFAA, graphite-furnace atomic absorption spectrometry; <, less than; DCP, direct current plasma; IC, ion chromatography]

\begin{tabular}{|c|c|c|c|c|c|c|c|c|}
\hline Analyte & $\begin{array}{l}\text { Reported- } \\
\text { value } \\
\text { units }\end{array}$ & $\begin{array}{c}\text { Date } \\
\text { (month- } \\
\text { year) }\end{array}$ & $\begin{array}{c}\text { USGS } \\
\text { standard } \\
\text { reference } \\
\text { sample }\end{array}$ & $\begin{array}{c}\text { Metro } \\
\text { District } \\
\text { value }\end{array}$ & $\begin{array}{l}\text { USGS } \\
\text { NWOL } \\
\text { value }\end{array}$ & $\begin{array}{c}\text { Most } \\
\text { probable } \\
\text { value }\end{array}$ & $\begin{array}{l}\text { Metro District } \\
\text { analytical } \\
\text { method }\end{array}$ & $\begin{array}{c}\text { NWOL } \\
\text { analytical } \\
\text { method }\end{array}$ \\
\hline $\mathrm{Ca}$ & $\mathrm{mg} / \mathrm{L}$ & Feb-98 & P29 & -- & 1.81 & 1.84 & na & ICP \\
\hline $\mathrm{Ca}$ & $\mathrm{mg} / \mathrm{L}$ & Feb-98 & $\mathrm{T} 151$ & -- & 37.9 & 37.9 & na & ICP \\
\hline $\mathrm{Ca}$ & $\mathrm{mg} / \mathrm{L}$ & Jul-98 & M146 & -- & 26.8 & 26.3 & na & ICP \\
\hline $\mathrm{Ca}$ & $\mathrm{mg} / \mathrm{L}$ & Jul-98 & P30 & -- & 0.13 & 0.13 & na & ICP \\
\hline $\mathrm{Ca}$ & $\mathrm{mg} / \mathrm{L}$ & Jul-98 & $\mathrm{T} 153$ & -- & 27.6 & 27.5 & na & ICP \\
\hline $\mathrm{Ca}$ & $\mathrm{mg} / \mathrm{L}$ & Dec-98 & M148 & -- & 5.85 & 5.90 & na & ICP \\
\hline $\mathrm{Ca}$ & $\mathrm{mg} / \mathrm{L}$ & Dec-98 & T155 & 49 & 42.3 & 42.0 & ICP & ICP \\
\hline $\mathrm{Ca}$ & $\mathrm{mg} / \mathrm{L}$ & Aug-99 & GWM4 & -- & 51.2 & 50.1 & na & ICP \\
\hline $\mathrm{Ca}$ & $\mathrm{mg} / \mathrm{L}$ & Aug-99 & GWT5 & -- & 50.7 & 50.8 & na & ICP \\
\hline $\mathrm{Ca}$ & $\mathrm{mg} / \mathrm{L}$ & Aug-99 & M150 & 7 & 6.68 & 6.82 & na & ICP \\
\hline $\mathrm{Ca}$ & $\mathrm{mg} / \mathrm{L}$ & Aug-99 & P32 & -- & 1.39 & 1.40 & na & ICP \\
\hline $\mathrm{Ca}$ & $\mathrm{mg} / \mathrm{L}$ & Aug-99 & $\mathrm{T} 157$ & 6 & 6.12 & 6.19 & na & ICP \\
\hline $\mathrm{Cd}$ & $\mu \mathrm{g} / \mathrm{L}$ & Feb-93 & $\mathrm{T} 121$ & 7.1 & 7.3 & 7.17 & GFAA & GFAA \\
\hline $\mathrm{Cd}$ & $\mu \mathrm{g} / \mathrm{L}$ & Aug-93 & $\mathrm{T} 123$ & 7.8 & 5.9 & 5.86 & GFAA & GFAA \\
\hline $\mathrm{Cd}$ & $\mu \mathrm{g} / \mathrm{L}$ & Aug-93 & T125 & 11.6 & 7.20 & 7.20 & GFAA & GFAA \\
\hline $\mathrm{Cd}$ & $\mu \mathrm{g} / \mathrm{L}$ & Jan-94 & $\mathrm{T} 127$ & 8.6 & 8.56 & 8.34 & GFAA & GFAA \\
\hline $\mathrm{Cd}$ & $\mu \mathrm{g} / \mathrm{L}$ & Aug-94 & T129 & 0.3 & 0.32 & 0.34 & GFAA & GFAA \\
\hline $\mathrm{Cd}$ & $\mu \mathrm{g} / \mathrm{L}$ & Jan-95 & $\mathrm{T} 131$ & 5.9 & 6.40 & 6.10 & GFAA & GFAA \\
\hline $\mathrm{Cd}$ & $\mu \mathrm{g} / \mathrm{L}$ & Jan-95 & $\mathrm{T} 133$ & 23.0 & 22.5 & 23.0 & GFAA & GFAA \\
\hline $\mathrm{Cd}$ & $\mu \mathrm{g} / \mathrm{L}$ & Jul-95 & $\mathrm{T} 135$ & -- & 49.1 & 50.5 & na & ICP-MS \\
\hline $\mathrm{Cd}$ & $\mu \mathrm{g} / \mathrm{L}$ & Feb-96 & $\mathrm{T} 137$ & -- & 6.36 & 6.80 & na & ICP-MS \\
\hline $\mathrm{Cd}$ & $\mu \mathrm{g} / \mathrm{L}$ & Aug-96 & T139 & 7.8 & 7.40 & 7.50 & GFAA & ICP-MS \\
\hline $\mathrm{Cd}$ & $\mu \mathrm{g} / \mathrm{L}$ & Aug-96 & $\mathrm{T} 141$ & 8.1 & 8.00 & 8.20 & GFAA & ICP-MS \\
\hline $\mathrm{Cd}$ & $\mu \mathrm{g} / \mathrm{L}$ & Jan-97 & $\mathrm{T} 143$ & -- & 20.07 & 19.1 & na & GFAA \\
\hline $\mathrm{Cd}$ & $\mu \mathrm{g} / \mathrm{L}$ & Jan-97 & $\mathrm{T} 145$ & -- & 9.79 & 9.33 & na & GFAA \\
\hline $\mathrm{Cd}$ & $\mu \mathrm{g} / \mathrm{L}$ & Aug-97 & $\mathrm{T} 147$ & 17.0 & 15.4 & 15.9 & GFAA & ICP-MS \\
\hline $\mathrm{Cd}$ & $\mu \mathrm{g} / \mathrm{L}$ & Aug-97 & T149 & 2.2 & 2.30 & 2.18 & GFAA & GFAA \\
\hline $\mathrm{Cd}$ & $\mu \mathrm{g} / \mathrm{L}$ & Feb-98 & $\mathrm{T} 151$ & 26.8 & 26.6 & 26.8 & GFAA & ICP-MS \\
\hline $\mathrm{Cd}$ & $\mu \mathrm{g} / \mathrm{L}$ & Jul-98 & $\mathrm{T} 153$ & -- & 16.0 & 16.0 & na & ICP-MS \\
\hline $\mathrm{Cd}$ & $\mu \mathrm{g} / \mathrm{L}$ & Dec-98 & $\mathrm{T} 155$ & 6.5 & 11.0 & 11.4 & GFAA & ICP-MS \\
\hline $\mathrm{Cd}$ & $\mu \mathrm{g} / \mathrm{L}$ & Aug-99 & $\mathrm{T} 157$ & 7.2 & 6.00 & 5.80 & na & ICP-MS \\
\hline $\mathrm{Cl}$ & $\mathrm{mg} / \mathrm{L}$ & Aug-93 & M126 & 25 & 20.2 & 20.7 & Colorimetry & Colorimetry \\
\hline $\mathrm{Cl}$ & $\mathrm{mg} / \mathrm{L}$ & Jan-94 & M128 & 98 & 93.8 & 98.2 & Colorimetry & IC \\
\hline $\mathrm{Cl}$ & $\mathrm{mg} / \mathrm{L}$ & Feb-94 & P21 & -- & 4.11 & 3.90 & na & $\mathrm{IC}$ \\
\hline $\mathrm{Cl}$ & $\mathrm{mg} / \mathrm{L}$ & Aug-94 & M130 & 26 & 21.6 & 21.4 & Colorimetry & $\mathrm{IC}$ \\
\hline $\mathrm{Cl}$ & $\mathrm{mg} / \mathrm{L}$ & Aug-94 & P22 & -- & 2.96 & 2.92 & na & $\mathrm{IC}$ \\
\hline $\mathrm{Cl}$ & $\mathrm{mg} / \mathrm{L}$ & Jan-95 & M132 & 56 & 55.9 & 55.7 & Colorimetry & $\mathrm{IC}$ \\
\hline $\mathrm{Cl}$ & $\mathrm{mg} / \mathrm{L}$ & Jan-95 & $\mathrm{P} 23$ & -- & 0.280 & 0.310 & na & IC \\
\hline $\mathrm{Cl}$ & $\mathrm{mg} / \mathrm{L}$ & Jul-95 & M134 & -- & 64.6 & 65.0 & na & $\mathrm{IC}$ \\
\hline $\mathrm{Cl}$ & $\mathrm{mg} / \mathrm{L}$ & Jul-95 & P24 & -- & 1.19 & 1.20 & na & IC \\
\hline $\mathrm{Cl}$ & $\mathrm{mg} / \mathrm{L}$ & Feb-96 & M136 & 89 & 93.23 & 92.0 & Colorimetry & IC \\
\hline $\mathrm{Cl}$ & $\mathrm{mg} / \mathrm{L}$ & Feb-96 & P25 & -- & 1.27 & 1.30 & na & $\mathrm{IC}$ \\
\hline $\mathrm{Cl}$ & $\mathrm{mg} / \mathrm{L}$ & Apr-96 & M138 & 42 & 33.1 & 33.4 & Colorimetry & IC \\
\hline $\mathrm{Cl}$ & $\mathrm{mg} / \mathrm{L}$ & Jun-96 & P26 & -- & 7.93 & 7.79 & na & $\mathrm{IC}$ \\
\hline $\mathrm{Cl}$ & $\mathrm{mg} / \mathrm{L}$ & Jan-97 & M140 & -- & 24.7 & 25.8 & na & $\mathrm{IC}$ \\
\hline $\mathrm{Cl}$ & $\mathrm{mg} / \mathrm{L}$ & Jan-97 & $\mathrm{P} 27$ & -- & 1.20 & 1.20 & na & $\mathrm{IC}$ \\
\hline $\mathrm{Cl}$ & $\mathrm{mg} / \mathrm{L}$ & Aug-97 & M142 & 133 & 135 & 132 & Colorimetry & IC \\
\hline $\mathrm{Cl}$ & $\mathrm{mg} / \mathrm{L}$ & Aug-97 & P28 & -- & 3.33 & 3.30 & na & IC \\
\hline $\mathrm{Cl}$ & $\mathrm{mg} / \mathrm{L}$ & Feb-98 & GWM2 & 47 & 47.2 & 48.9 & Colorimetry & $\mathrm{IC}$ \\
\hline $\mathrm{Cl}$ & $\mathrm{mg} / \mathrm{L}$ & Feb-98 & M144 & 77 & 76.2 & 77.0 & Colorimetry & $\mathrm{IC}$ \\
\hline $\mathrm{Cl}$ & $\mathrm{mg} / \mathrm{L}$ & Feb-98 & P29 & -- & 0.15 & 0.20 & na & $\mathrm{IC}$ \\
\hline $\mathrm{Cl}$ & $\mathrm{mg} / \mathrm{L}$ & Jul-98 & M146 & -- & 44 & 46.1 & na & $\mathrm{IC}$ \\
\hline $\mathrm{Cl}$ & $\mathrm{mg} / \mathrm{L}$ & Jul-98 & P30 & -- & 0.18 & 0.23 & na & $\mathrm{IC}$ \\
\hline $\mathrm{Cl}$ & $\mathrm{mg} / \mathrm{L}$ & Dec-98 & M148 & 46 & 44.6 & 46.0 & Colorimetry & IC \\
\hline
\end{tabular}


Appendix 8. Performance-evaluation data from the U.S. Geological Survey Standard Reference Program for the Metro Wastewater Reclamation District Laboratory (1993-1998) and the U.S. Geological Survey National Water Quality Laboratory (1993-1999).-Continued

[All abbreviations explained in appendix 2; USGS, U.S. Geological Survey; Metro District, Metro Wastewater Reclamation District Laboratory; NWQL, National Water Quality Laboratory; $\mu \mathrm{g} / \mathrm{L}$, micrograms per liter; $\mathrm{mg} / \mathrm{L}$, milligrams per liter; $\mu \mathrm{S} / \mathrm{cm}$, microsiemens per centimeter at 25 degrees Celsius; ICP, inductively coupled plasma; MS, mass spectrometry; --; no data; na, not applicable; AA, atomic absorption spectrometry; GFAA, graphite-furnace atomic absorption spectrometry; <, less than; DCP, direct current plasma; IC, ion chromatography]

\begin{tabular}{|c|c|c|c|c|c|c|c|c|}
\hline Analyte & $\begin{array}{l}\text { Reported- } \\
\text { value } \\
\text { units }\end{array}$ & $\begin{array}{c}\text { Date } \\
\text { (month- } \\
\text { year) }\end{array}$ & $\begin{array}{c}\text { USGS } \\
\text { standard } \\
\text { reference } \\
\text { sample }\end{array}$ & $\begin{array}{c}\text { Metro } \\
\text { District } \\
\text { value }\end{array}$ & $\begin{array}{l}\text { USGS } \\
\text { NWOL } \\
\text { value }\end{array}$ & $\begin{array}{c}\text { Most } \\
\text { probable } \\
\text { value }\end{array}$ & $\begin{array}{l}\text { Metro District } \\
\text { analytical } \\
\text { method }\end{array}$ & $\begin{array}{c}\text { NWQL } \\
\text { analytical } \\
\text { method }\end{array}$ \\
\hline $\mathrm{Cl}$ & $\mathrm{mg} / \mathrm{L}$ & Aug-99 & M150 & 18 & 19.5 & 17.0 & na & $\mathrm{IC}$ \\
\hline $\mathrm{Cl}$ & $\mathrm{mg} / \mathrm{L}$ & Aug-99 & P32 & -- & 1.66 & 1.64 & na & IC \\
\hline $\mathrm{Co}$ & $\mu \mathrm{g} / \mathrm{L}$ & Jan-94 & T127 & -- & 11.3 & 11.6 & na & GFAA \\
\hline $\mathrm{Co}$ & $\mu \mathrm{g} / \mathrm{L}$ & Jan-95 & T133 & -- & 19.9 & 20.0 & na & ICP-MS \\
\hline $\mathrm{Co}$ & $\mu \mathrm{g} / \mathrm{L}$ & Jul-95 & $\mathrm{T} 135$ & -- & 39.6 & 40.0 & na & ICP-MS \\
\hline $\mathrm{Co}$ & $\mu \mathrm{g} / \mathrm{L}$ & Aug-96 & T139 & -- & 6.3 & 6.7 & na & ICP-MS \\
\hline $\mathrm{Co}$ & $\mu \mathrm{g} / \mathrm{L}$ & Aug-96 & $\mathrm{T} 141$ & -- & 6.10 & 6.50 & na & ICP-MS \\
\hline $\mathrm{Co}$ & $\mu \mathrm{g} / \mathrm{L}$ & Jan-97 & $\mathrm{T} 143$ & -- & 17.4 & 17.0 & na & GFAA \\
\hline $\mathrm{Co}$ & $\mu \mathrm{g} / \mathrm{L}$ & Jan-97 & $\mathrm{T} 145$ & -- & 10.0 & 10.0 & na & ICP-MS \\
\hline $\mathrm{Co}$ & $\mu \mathrm{g} / \mathrm{L}$ & Aug-99 & $\mathrm{T} 157$ & -- & 4.23 & 4.03 & na & ICP-MS \\
\hline $\mathrm{Cr}$ & $\mu \mathrm{g} / \mathrm{L}$ & Jan-94 & T127 & $<20$ & 10.5 & 11.5 & ICP & ICP \\
\hline $\mathrm{Cr}$ & $\mu \mathrm{g} / \mathrm{L}$ & Aug-94 & T129 & -- & 0.60 & 0.68 & na & ICP-MS \\
\hline $\mathrm{Cr}$ & $\mu \mathrm{g} / \mathrm{L}$ & Jan-95 & T131 & $<20$ & 17.3 & 18.6 & $\mathrm{ICP}$ & GFAA \\
\hline $\mathrm{Cr}$ & $\mu \mathrm{g} / \mathrm{L}$ & Jan-95 & T133 & 40 & 36.2 & 38.0 & ICP & GFAA \\
\hline $\mathrm{Cr}$ & $\mu \mathrm{g} / \mathrm{L}$ & Jul-95 & T135 & - & 80.9 & 79.0 & na & ICP \\
\hline $\mathrm{Cr}$ & $\mu \mathrm{g} / \mathrm{L}$ & Feb-96 & $\mathrm{T} 137$ & 20 & 20.5 & 19.4 & ICP & GFAA \\
\hline $\mathrm{Cr}$ & $\mu \mathrm{g} / \mathrm{L}$ & Aug-96 & T139 & -- & 7.50 & 7.75 & na & ICP-MS \\
\hline $\mathrm{Cr}$ & $\mu \mathrm{g} / \mathrm{L}$ & Aug-96 & $\mathrm{T} 141$ & -- & 14.0 & 15.4 & na & ICP-MS \\
\hline $\mathrm{Cr}$ & $\mu \mathrm{g} / \mathrm{L}$ & Jan-97 & T143 & -- & 35.5 & 37.0 & na & ICP-MS \\
\hline $\mathrm{Cr}$ & $\mu \mathrm{g} / \mathrm{L}$ & Jan-97 & $\mathrm{T} 145$ & -- & 15.5 & 15.3 & na & GFAA \\
\hline $\mathrm{Cr}$ & $\mu \mathrm{g} / \mathrm{L}$ & Aug-97 & $\mathrm{T} 147$ & $<20$ & 11.4 & 12.8 & ICP & ICP-MS \\
\hline $\mathrm{Cu}$ & $\mu \mathrm{g} / \mathrm{L}$ & Jan-95 & $\mathrm{T} 133$ & 82 & 84.4 & 85.3 & GFAA & ICP \\
\hline $\mathrm{Cu}$ & $\mu \mathrm{g} / \mathrm{L}$ & Jul-95 & $\mathrm{T} 135$ & -- & 61.9 & 62.0 & na & ICP-MS \\
\hline $\mathrm{Cu}$ & $\mu \mathrm{g} / \mathrm{L}$ & Feb-96 & T137 & 2 & 2.14 & 1.9 & GFAA & ICP-MS \\
\hline $\mathrm{Cu}$ & $\mu \mathrm{g} / \mathrm{L}$ & Aug-96 & T139 & 13 & 12.8 & 13.0 & GFAA & ICP-MS \\
\hline $\mathrm{Cu}$ & $\mu \mathrm{g} / \mathrm{L}$ & Aug-96 & $\mathrm{T} 141$ & 19 & 18.5 & 18.0 & GFAA & GFAA \\
\hline $\mathrm{Cu}$ & $\mu \mathrm{g} / \mathrm{L}$ & Jan-97 & T143 & - & 22.3 & 22.2 & na & GFAA \\
\hline $\mathrm{Cu}$ & $\mu \mathrm{g} / \mathrm{L}$ & Jan-97 & $\mathrm{T} 145$ & -- & 11.1 & 11.0 & na & GFAA \\
\hline $\mathrm{Cu}$ & $\mu \mathrm{g} / \mathrm{L}$ & Aug-97 & T147 & 10 & 11.0 & 11.4 & GFAA & ICP-MS \\
\hline $\mathrm{Cu}$ & $\mu \mathrm{g} / \mathrm{L}$ & Aug-97 & $\mathrm{T} 149$ & 7 & 7.60 & 8.00 & GFAA & ICP-MS \\
\hline $\mathrm{Cu}$ & $\mu \mathrm{g} / \mathrm{L}$ & Feb-98 & $\mathrm{T} 151$ & 32 & 32.7 & 33.0 & GFAA & ICP-MS \\
\hline $\mathrm{Cu}$ & $\mu \mathrm{g} / \mathrm{L}$ & Jul-98 & $\mathrm{T} 153$ & -- & 23.7 & 24.0 & na & ICP-MS \\
\hline $\mathrm{Cu}$ & $\mu \mathrm{g} / \mathrm{L}$ & Dec-98 & $\mathrm{T} 155$ & 36 & 37.9 & 38.0 & GFAA & ICP-MS \\
\hline $\mathrm{Cu}$ & $\mu \mathrm{g} / \mathrm{L}$ & Aug-99 & GWT5 & 2 & 2.35 & 2.51 & GFAA & ICP-MS \\
\hline $\mathrm{Cu}$ & $\mu \mathrm{g} / \mathrm{L}$ & Aug-99 & $\mathrm{T} 157$ & 25 & 25.2 & 24.8 & GFAA & ICP-MS \\
\hline TDS (ROE) & $\mathrm{mg} / \mathrm{L}$ & Jan-94 & M128 & 718 & 684 & 689 & Gravimetric & Gravimetric \\
\hline TDS (ROE) & $\mathrm{mg} / \mathrm{L}$ & Aug-94 & M130 & 199 & 193 & 200 & Gravimetric & Gravimetric \\
\hline TDS (ROE) & $\mathrm{mg} / \mathrm{L}$ & Jan-95 & M132 & 271 & 264 & 277 & Gravimetric & Gravimetric \\
\hline
\end{tabular}


Appendix 8. Performance-evaluation data from the U.S. Geological Survey Standard Reference Program for the Metro Wastewater Reclamation District Laboratory (1993-1998) and the U.S. Geological Survey National Water Quality Laboratory (1993-1999).-Continued

[All abbreviations explained in appendix 2; USGS, U.S. Geological Survey; Metro District, Metro Wastewater Reclamation District Laboratory; NWQL, National Water Quality Laboratory; $\mu \mathrm{g} / \mathrm{L}$, micrograms per liter; $\mathrm{mg} / \mathrm{L}$, milligrams per liter; $\mu \mathrm{S} / \mathrm{cm}$, microsiemens per centimeter at 25 degrees Celsius; ICP, inductively coupled plasma; MS, mass spectrometry; --; no data; na, not applicable; AA, atomic absorption spectrometry; GFAA, graphite-furnace atomic absorption spectrometry; <, less than; DCP, direct current plasma; IC, ion chromatography]

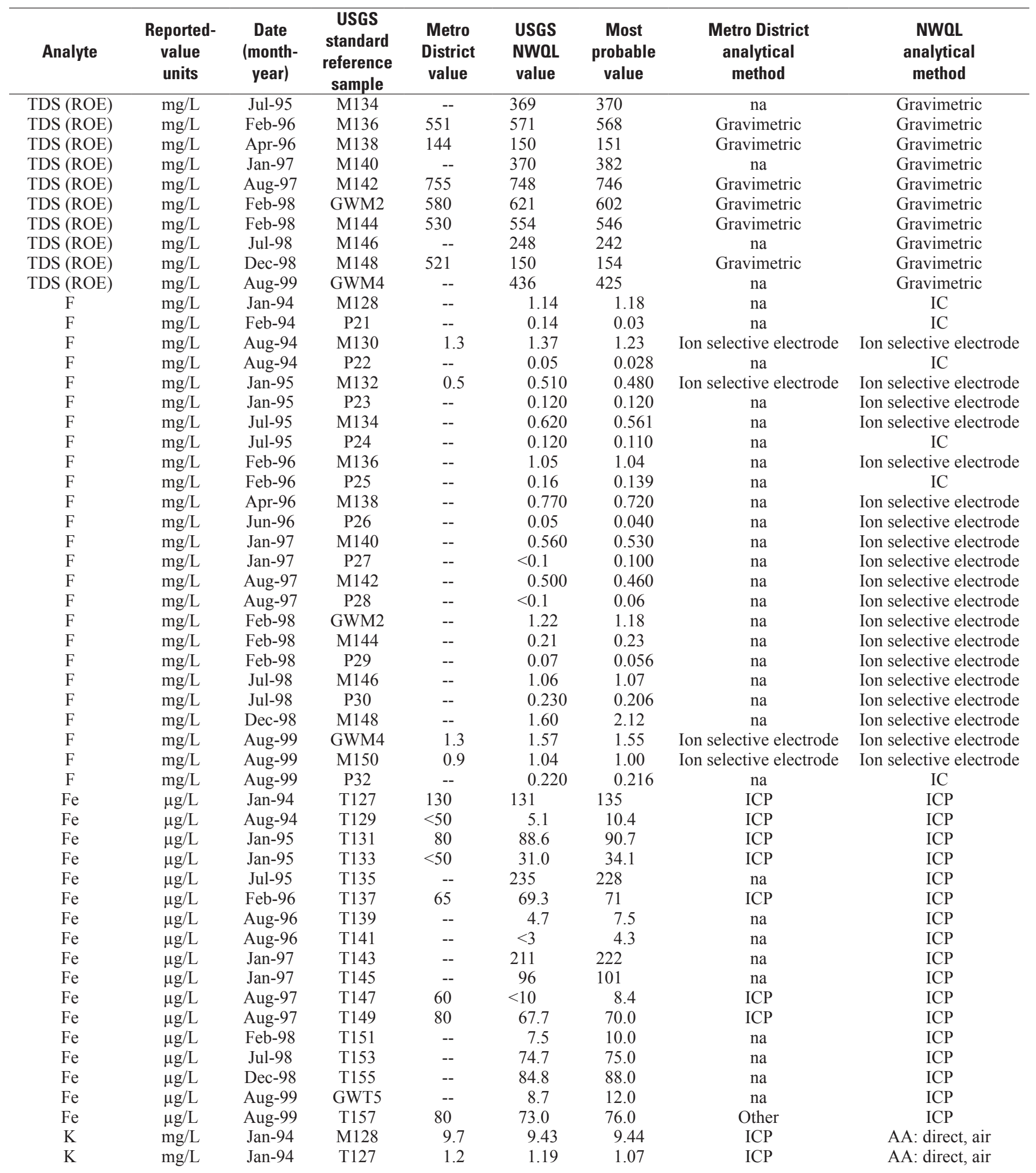


Appendix 8. Performance-evaluation data from the U.S. Geological Survey Standard Reference Program for the Metro Wastewater Reclamation District Laboratory (1993-1998) and the U.S. Geological Survey National Water Quality Laboratory (1993-1999).-Continued

[All abbreviations explained in appendix 2; USGS, U.S. Geological Survey; Metro District, Metro Wastewater Reclamation District Laboratory; NWQL, National Water Quality Laboratory; $\mu \mathrm{g} / \mathrm{L}$, micrograms per liter; $\mathrm{mg} / \mathrm{L}$, milligrams per liter; $\mu \mathrm{S} / \mathrm{cm}$, microsiemens per centimeter at 25 degrees Celsius; ICP, inductively coupled plasma; MS, mass spectrometry; --; no data; na, not applicable; AA, atomic absorption spectrometry; GFAA, graphite-furnace atomic absorption spectrometry; <, less than; DCP, direct current plasma; IC, ion chromatography]

\begin{tabular}{|c|c|c|c|c|c|c|c|c|}
\hline Analyte & $\begin{array}{l}\text { Reported- } \\
\text { value } \\
\text { units }\end{array}$ & $\begin{array}{c}\text { Date } \\
\text { (month- } \\
\text { year) }\end{array}$ & $\begin{array}{c}\text { USGS } \\
\text { standard } \\
\text { reference } \\
\text { sample }\end{array}$ & $\begin{array}{c}\text { Metro } \\
\text { District } \\
\text { value }\end{array}$ & $\begin{array}{l}\text { USGS } \\
\text { NWOL } \\
\text { value }\end{array}$ & $\begin{array}{c}\text { Most } \\
\text { probable } \\
\text { value }\end{array}$ & $\begin{array}{l}\text { Metro District } \\
\text { analytical } \\
\text { method }\end{array}$ & $\begin{array}{c}\text { NWQL } \\
\text { analytical } \\
\text { method }\end{array}$ \\
\hline $\mathrm{K}$ & $\mathrm{mg} / \mathrm{L}$ & Aug-94 & M130 & 3 & 2.88 & 3.00 & $\mathrm{ICP}$ & AA: direct, air \\
\hline $\mathrm{K}$ & $\mathrm{mg} / \mathrm{L}$ & Aug-94 & P22 & -- & 0.23 & 0.203 & na & AA: direct, air \\
\hline $\mathrm{K}$ & $\mathrm{mg} / \mathrm{L}$ & Aug-94 & $\mathrm{T} 129$ & 3 & 2.92 & 3.00 & ICP & AA: direct, air \\
\hline $\mathrm{K}$ & $\mathrm{mg} / \mathrm{L}$ & Jan-95 & $\mathrm{T} 131$ & 2.4 & 2.25 & 2.39 & $\mathrm{ICP}$ & AA: direct, air \\
\hline $\mathrm{K}$ & $\mathrm{mg} / \mathrm{L}$ & Jan-95 & $\mathrm{T} 133$ & 1.0 & 0.87 & 1.00 & ICP & AA: direct, air \\
\hline $\mathrm{K}$ & $\mathrm{mg} / \mathrm{L}$ & Jul-95 & M134 & -- & 2.39 & 2.40 & na & AA: direct, air \\
\hline $\mathrm{K}$ & $\mathrm{mg} / \mathrm{L}$ & Jul-95 & P24 & -- & 0.12 & 0.118 & na & AA: direct, air \\
\hline $\mathrm{K}$ & $\mathrm{mg} / \mathrm{L}$ & Jul-95 & $\mathrm{T} 135$ & -- & 0.90 & 0.96 & na & AA: direct, air \\
\hline $\mathrm{K}$ & $\mathrm{mg} / \mathrm{L}$ & Feb-96 & M136 & 7 & 6.69 & 6.53 & $\mathrm{ICP}$ & AA: direct, air \\
\hline $\mathrm{K}$ & $\mathrm{mg} / \mathrm{L}$ & Aug-96 & $\mathrm{T} 141$ & 2 & 2.34 & 2.32 & ICP & AA: direct, air \\
\hline $\mathrm{K}$ & $\mathrm{mg} / \mathrm{L}$ & Jan-97 & M140 & -- & 2.57 & 2.58 & na & AA: direct, air \\
\hline $\mathrm{K}$ & $\mathrm{mg} / \mathrm{L}$ & Jan-97 & P27 & -- & 0.33 & 0.336 & na & AA: direct, air \\
\hline $\mathrm{K}$ & $\mathrm{mg} / \mathrm{L}$ & Jan-97 & $\mathrm{T} 143$ & -- & 2.55 & 2.50 & na & AA: direct, air \\
\hline $\mathrm{K}$ & $\mathrm{mg} / \mathrm{L}$ & Jan-97 & $\mathrm{T} 145$ & -- & 2.17 & 2.13 & na & AA: direct, air \\
\hline $\mathrm{K}$ & $\mathrm{mg} / \mathrm{L}$ & Aug-97 & M142 & 6 & 5.60 & 5.72 & ICP & AA: direct, air \\
\hline $\mathrm{K}$ & $\mathrm{mg} / \mathrm{L}$ & Aug-97 & P28 & -- & 0.14 & 0.14 & na & AA: direct, air \\
\hline $\mathrm{K}$ & $\mathrm{mg} / \mathrm{L}$ & Aug-97 & $\mathrm{T} 147$ & 3 & 3.48 & 3.52 & ICP & AA: direct, air \\
\hline $\mathrm{K}$ & $\mathrm{mg} / \mathrm{L}$ & Aug-97 & T149 & 2 & 1.88 & 2.00 & ICP & AA: direct, air \\
\hline $\mathrm{K}$ & $\mathrm{mg} / \mathrm{L}$ & Feb-98 & GWM2 & -- & 4.44 & 4.46 & na & AA: direct sir \\
\hline $\mathrm{K}$ & $\mathrm{mg} / \mathrm{L}$ & Feb-98 & M144 & -- & 3.56 & 3.60 & na & AA: direct, air \\
\hline $\mathrm{K}$ & $\mathrm{mg} / \mathrm{L}$ & Feb-98 & P29 & -- & 0.37 & 0.37 & na & AA: direct, air \\
\hline $\mathrm{K}$ & $\mathrm{mg} / \mathrm{L}$ & Aug-99 & GWT5 & -- & 1.78 & 1.85 & na & $\begin{array}{l}\text { AA: direct, } \\
\text { nitrous oxide }\end{array}$ \\
\hline K & $\mathrm{mg} / \mathrm{L}$ & Aug-99 & M150 & -- & 1.09 & 1.12 & na & $\begin{array}{l}\text { AA: direct, } \\
\text { nitrous oxide }\end{array}$ \\
\hline K & $\mathrm{mg} / \mathrm{L}$ & Aug-99 & P32 & -- & 0.36 & 0.399 & na & $\begin{array}{l}\text { AA: direct, } \\
\text { nitrous oxide }\end{array}$ \\
\hline K & $\mathrm{mg} / \mathrm{L}$ & Aug-99 & $\mathrm{T} 157$ & 2.00 & 2.47 & 2.51 & ICP & $\begin{array}{l}\text { AA: direct, } \\
\text { nitrous oxide }\end{array}$ \\
\hline $\mathrm{Li}$ & $\mu \mathrm{g} / \mathrm{L}$ & Jan-94 & $\mathrm{T} 127$ & -- & 24.0 & 24.0 & na & ICP \\
\hline $\mathrm{Li}$ & $\mu \mathrm{g} / \mathrm{L}$ & Aug-94 & T129 & -- & 17.0 & 18.0 & na & ICP \\
\hline $\mathrm{Li}$ & $\mu \mathrm{g} / \mathrm{L}$ & Jan-95 & $\mathrm{T} 131$ & -- & 19.2 & 17.0 & na & ICP \\
\hline $\mathrm{Li}$ & $\mu \mathrm{g} / \mathrm{L}$ & Jan-95 & T133 & -- & 52.4 & 51.0 & na & ICP \\
\hline $\mathrm{Li}$ & $\mu \mathrm{g} / \mathrm{L}$ & Jul-95 & $\mathrm{T} 135$ & -- & 73.6 & 73.7 & na & ICP \\
\hline $\mathrm{Li}$ & $\mu \mathrm{g} / \mathrm{L}$ & Feb-96 & $\mathrm{T} 137$ & -- & 8.67 & 8.70 & na & ICP \\
\hline $\mathrm{Li}$ & $\mu \mathrm{g} / \mathrm{L}$ & Aug-96 & T139 & -- & 19.3 & 18.7 & na & ICP \\
\hline $\mathrm{Li}$ & $\mu \mathrm{g} / \mathrm{L}$ & Aug-96 & $\mathrm{T} 141$ & -- & 22.0 & 21.4 & na & ICP \\
\hline $\mathrm{Li}$ & $\mu \mathrm{g} / \mathrm{L}$ & Jan-97 & T143 & -- & 16.6 & 18.0 & na & ICP \\
\hline
\end{tabular}


Appendix 8. Performance-evaluation data from the U.S. Geological Survey Standard Reference Program for the Metro Wastewater Reclamation District Laboratory (1993-1998) and the U.S. Geological Survey National Water Quality Laboratory (1993-1999).—Continued

[All abbreviations explained in appendix 2; USGS, U.S. Geological Survey; Metro District, Metro Wastewater Reclamation District Laboratory; NWQL, National Water Quality Laboratory; $\mu \mathrm{g} / \mathrm{L}$, micrograms per liter; $\mathrm{mg} / \mathrm{L}$, milligrams per liter; $\mu \mathrm{S} / \mathrm{cm}$, microsiemens per centimeter at 25 degrees Celsius; ICP, inductively coupled plasma; MS, mass spectrometry; --; no data; na, not applicable; AA, atomic absorption spectrometry; GFAA, graphite-furnace atomic absorption spectrometry; <, less than; DCP, direct current plasma; IC, ion chromatography]

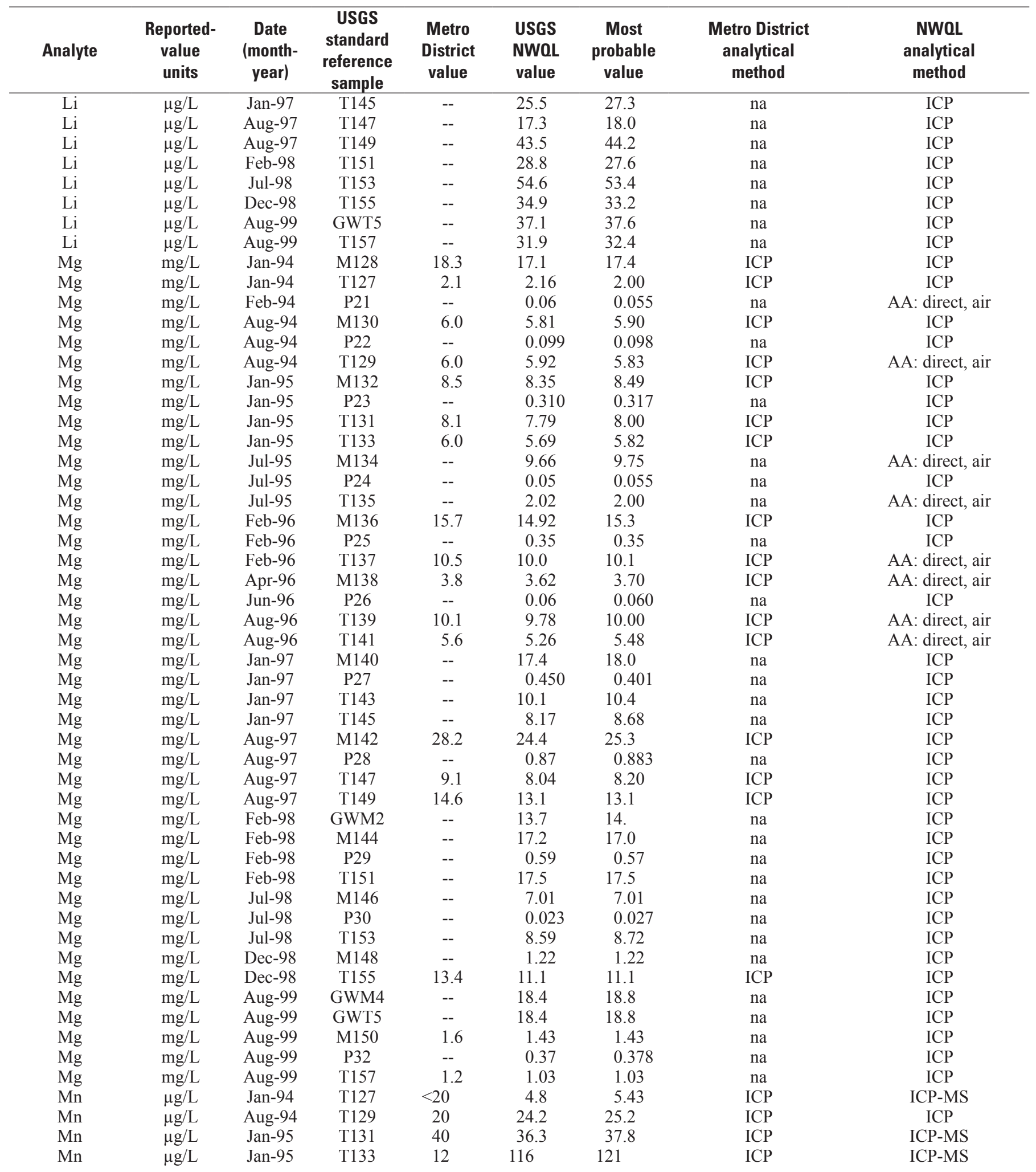


Appendix 8. Performance-evaluation data from the U.S. Geological Survey Standard Reference Program for the Metro Wastewater Reclamation District Laboratory (1993-1998) and the U.S. Geological Survey National Water Quality Laboratory (1993-1999).-Continued

[All abbreviations explained in appendix 2; USGS, U.S. Geological Survey; Metro District, Metro Wastewater Reclamation District Laboratory; NWQL, National Water Quality Laboratory; $\mu \mathrm{g} / \mathrm{L}$, micrograms per liter; $\mathrm{mg} / \mathrm{L}$, milligrams per liter; $\mu \mathrm{S} / \mathrm{cm}$, microsiemens per centimeter at 25 degrees Celsius; ICP, inductively coupled plasma; MS, mass spectrometry; --; no data; na, not applicable; AA, atomic absorption spectrometry; GFAA, graphite-furnace atomic absorption spectrometry; <, less than; DCP, direct current plasma; IC, ion chromatography]

\begin{tabular}{|c|c|c|c|c|c|c|c|c|}
\hline Analyte & $\begin{array}{l}\text { Reported- } \\
\text { value } \\
\text { units }\end{array}$ & $\begin{array}{c}\text { Date } \\
\text { (month- } \\
\text { year) }\end{array}$ & $\begin{array}{c}\text { USGS } \\
\text { standard } \\
\text { reference } \\
\text { sample }\end{array}$ & $\begin{array}{c}\text { Metro } \\
\text { District } \\
\text { value }\end{array}$ & $\begin{array}{l}\text { USGS } \\
\text { NWOL } \\
\text { value }\end{array}$ & $\begin{array}{c}\text { Most } \\
\text { probable } \\
\text { value }\end{array}$ & $\begin{array}{l}\text { Metro District } \\
\text { analytical } \\
\text { method }\end{array}$ & $\begin{array}{c}\text { NWQL } \\
\text { analytical } \\
\text { method }\end{array}$ \\
\hline $\mathrm{Mn}$ & $\mu \mathrm{g} / \mathrm{L}$ & Feb-96 & T137 & 100 & 89.37 & 98 & $\mathrm{ICP}$ & ICP-MS \\
\hline $\mathrm{Mn}$ & $\mu \mathrm{g} / \mathrm{L}$ & Aug-96 & T139 & - & 2.2 & 2.4 & na & ICP-MS \\
\hline $\mathrm{Mn}$ & $\mu \mathrm{g} / \mathrm{L}$ & Aug-96 & $\mathrm{T} 141$ & -- & 19.7 & 20.0 & na & ICP-MS \\
\hline $\mathrm{Mn}$ & $\mu \mathrm{g} / \mathrm{L}$ & Aug-97 & $\mathrm{T} 147$ & $<30$ & 15.9 & 17.2 & $\mathrm{ICP}$ & ICP-MS \\
\hline $\mathrm{Mn}$ & $\mu \mathrm{g} / \mathrm{L}$ & Aug-97 & $\mathrm{T} 149$ & $<30$ & 11.2 & 11.8 & ICP & ICP \\
\hline $\mathrm{Mn}$ & $\mu \mathrm{g} / \mathrm{L}$ & Feb-98 & $\mathrm{T} 151$ & -- & 12.8 & 13.0 & na & ICP-MS \\
\hline $\mathrm{Mn}$ & $\mu \mathrm{g} / \mathrm{L}$ & Jul-98 & $\mathrm{T} 153$ & -- & 72.5 & 74.5 & na & ICP-MS \\
\hline $\mathrm{Mn}$ & $\mu \mathrm{g} / \mathrm{L}$ & Dec-98 & $\mathrm{T} 155$ & -- & 48.0 & 50.9 & na & ICP-MS \\
\hline Mo & $\mu \mathrm{g} / \mathrm{L}$ & Jan-94 & T127 & -- & 0.46 & 1.25 & na & ICP-MS \\
\hline Mo & $\mu \mathrm{g} / \mathrm{L}$ & Feb-96 & T137 & -- & 8.9 & 8.9 & na & GFAA \\
\hline Mo & $\mu \mathrm{g} / \mathrm{L}$ & Aug-96 & T139 & -- & 14.7 & 14.9 & na & ICP-MS \\
\hline Mo & $\mu \mathrm{g} / \mathrm{L}$ & Aug-96 & $\mathrm{T} 141$ & -- & 2.2 & 2.1 & na & ICP-MS \\
\hline Mo & $\mu \mathrm{g} / \mathrm{L}$ & Jan-97 & $\mathrm{T} 143$ & -- & 34.4 & 36.1 & na & GFAA \\
\hline Mo & $\mu \mathrm{g} / \mathrm{L}$ & Jan-97 & T145 & -- & 8.67 & 9.23 & na & GFAA \\
\hline Mo & $\mu \mathrm{g} / \mathrm{L}$ & Aug-97 & $\mathrm{T} 147$ & $<30$ & 10.9 & 11.8 & ICP & GFAA \\
\hline Mo & $\mu \mathrm{g} / \mathrm{L}$ & Aug-97 & T149 & $<30$ & 1.14 & 1.25 & ICP & ICP-MS \\
\hline Mo & $\mu \mathrm{g} / \mathrm{L}$ & Feb-98 & $\mathrm{T} 151$ & -- & 29.2 & 29.0 & na & ICP-MS \\
\hline Mo & $\mu \mathrm{g} / \mathrm{L}$ & Jul-98 & $\mathrm{T} 153$ & -- & 155 & 154 & na & ICP-MS \\
\hline Mo & $\mu \mathrm{g} / \mathrm{L}$ & Dec-98 & $\mathrm{T} 155$ & -- & 25.2 & 25.2 & na & ICP-MS \\
\hline Mo & $\mu \mathrm{g} / \mathrm{L}$ & Aug-99 & GWT5 & -- & 9.76 & 9.27 & na & ICP-MS \\
\hline Mo & $\mu \mathrm{g} / \mathrm{L}$ & Aug-99 & T157 & -- & 9.5 & 13.0 & na & ICP-MS \\
\hline $\mathrm{Na}$ & $\mathrm{mg} / \mathrm{L}$ & Jan-94 & M128 & 128 & 121 & 126 & ICP & ICP \\
\hline $\mathrm{Na}$ & $\mathrm{mg} / \mathrm{L}$ & Jan-95 & $\mathrm{T} 133$ & 30 & 29.5 & 29.4 & $\mathrm{ICP}$ & $\mathrm{AA}$ : direct, air \\
\hline $\mathrm{Na}$ & $\mathrm{mg} / \mathrm{L}$ & Jul-95 & M134 & - & 60.4 & 60.7 & na & AA: direct, air \\
\hline $\mathrm{Na}$ & $\mathrm{mg} / \mathrm{L}$ & Jul-95 & P24 & -- & 0.27 & 0.246 & na & AA: direct, air \\
\hline $\mathrm{Na}$ & $\mathrm{mg} / \mathrm{L}$ & Jul-95 & $\mathrm{T} 135$ & -- & 30.4 & 30.8 & na & $\mathrm{AA}$ : direct, air \\
\hline $\mathrm{Na}$ & $\mathrm{mg} / \mathrm{L}$ & Feb-96 & M136 & 118 & 107.6 & 108 & ICP & AA: direct, air \\
\hline $\mathrm{Na}$ & $\mathrm{mg} / \mathrm{L}$ & Feb-96 & P25 & -- & 1.24 & 1.28 & na & $\mathrm{AA}$ : direct, air \\
\hline $\mathrm{Na}$ & $\mathrm{mg} / \mathrm{L}$ & Feb-96 & $\mathrm{T} 137$ & 22 & 20.7 & 22 & $\mathrm{ICP}$ & AA: direct, air \\
\hline $\mathrm{Na}$ & $\mathrm{mg} / \mathrm{L}$ & Apr-96 & M138 & 28 & 31.4 & 31.6 & ICP & AA: direct, air \\
\hline $\mathrm{Na}$ & $\mathrm{mg} / \mathrm{L}$ & Jun-96 & P26 & -- & 4.49 & 4.40 & na & ICP \\
\hline $\mathrm{Na}$ & $\mathrm{mg} / \mathrm{L}$ & Aug-96 & $\mathrm{T} 139$ & 88 & 91.1 & 90.9 & ICP & $\mathrm{AA}$ : direct, air \\
\hline $\mathrm{Na}$ & $\mathrm{mg} / \mathrm{L}$ & Aug-96 & $\mathrm{T} 141$ & 30 & 32.5 & 33.0 & $\mathrm{ICP}$ & ICP \\
\hline $\mathrm{Na}$ & $\mathrm{mg} / \mathrm{L}$ & Jan-97 & M140 & - & 38.2 & 39.0 & na & ICP \\
\hline $\mathrm{Na}$ & $\mathrm{mg} / \mathrm{L}$ & Jan-97 & P27 & -- & 1.34 & 1.34 & na & ICP \\
\hline $\mathrm{Na}$ & $\mathrm{mg} / \mathrm{L}$ & Jan-97 & T143 & -- & 33.6 & 34.0 & na & $\mathrm{ICP}$ \\
\hline $\mathrm{Na}$ & $\mathrm{mg} / \mathrm{L}$ & Jan-97 & $\mathrm{T} 145$ & -- & 40.2 & 41.2 & na & $\mathrm{ICP}$ \\
\hline $\mathrm{Na}$ & $\mathrm{mg} / \mathrm{L}$ & Aug-97 & M142 & 149 & 150 & 153 & $\mathrm{ICP}$ & $\mathrm{ICP}$ \\
\hline $\mathrm{Na}$ & $\mathrm{mg} / \mathrm{L}$ & Aug-97 & P28 & -- & 3.30 & 3.25 & na & ICP \\
\hline
\end{tabular}


Appendix 8. Performance-evaluation data from the U.S. Geological Survey Standard Reference Program for the Metro Wastewater Reclamation District Laboratory (1993-1998) and the U.S. Geological Survey National Water Quality Laboratory (1993-1999).—Continued

[All abbreviations explained in appendix 2; USGS, U.S. Geological Survey; Metro District, Metro Wastewater Reclamation District Laboratory; NWQL, National Water Quality Laboratory; $\mu \mathrm{g} / \mathrm{L}$, micrograms per liter; $\mathrm{mg} / \mathrm{L}$, milligrams per liter; $\mu \mathrm{S} / \mathrm{cm}$, microsiemens per centimeter at 25 degrees Celsius; ICP, inductively coupled plasma; MS, mass spectrometry; --; no data; na, not applicable; AA, atomic absorption spectrometry; GFAA, graphite-furnace atomic absorption spectrometry; <, less than; DCP, direct current plasma; IC, ion chromatography]

\begin{tabular}{|c|c|c|c|c|c|c|c|c|}
\hline Analyte & $\begin{array}{l}\text { Reported- } \\
\text { value } \\
\text { units }\end{array}$ & $\begin{array}{c}\text { Date } \\
\text { (month- } \\
\text { year) }\end{array}$ & $\begin{array}{c}\text { USGS } \\
\text { standard } \\
\text { reference } \\
\text { sample } \\
\end{array}$ & $\begin{array}{c}\text { Metro } \\
\text { District } \\
\text { value }\end{array}$ & $\begin{array}{l}\text { USGS } \\
\text { NWOL } \\
\text { value }\end{array}$ & $\begin{array}{c}\text { Most } \\
\text { probable } \\
\text { value }\end{array}$ & $\begin{array}{l}\text { Metro District } \\
\text { analytical } \\
\text { method }\end{array}$ & $\begin{array}{l}\text { NWOL } \\
\text { analytical } \\
\text { method }\end{array}$ \\
\hline $\mathrm{Na}$ & $\mathrm{mg} / \mathrm{L}$ & Aug-97 & T149 & 45 & 43.0 & 42.8 & ICP & ICP \\
\hline $\mathrm{Na}$ & $\mathrm{mg} / \mathrm{L}$ & Feb-98 & GWM2 & -- & 116 & 117 & na & ICP \\
\hline $\mathrm{Na}$ & $\mathrm{mg} / \mathrm{L}$ & Feb-98 & M144 & -- & 78.8 & 77.7 & na & ICP \\
\hline $\mathrm{Na}$ & $\mathrm{mg} / \mathrm{L}$ & Jul-98 & M146 & -- & 44.6 & 45.0 & na & $\mathrm{ICP}$ \\
\hline $\mathrm{Na}$ & $\mathrm{mg} / \mathrm{L}$ & Jul-98 & P30 & -- & 0.32 & 0.34 & na & ICP \\
\hline $\mathrm{Na}$ & $\mathrm{mg} / \mathrm{L}$ & Jul-98 & $\mathrm{T} 153$ & -- & 28.6 & 28.7 & na & ICP \\
\hline $\mathrm{Na}$ & $\mathrm{mg} / \mathrm{L}$ & Dec-98 & M148 & 31 & 32.5 & 31.5 & ICP & ICP \\
\hline $\mathrm{Na}$ & $\mathrm{mg} / \mathrm{L}$ & Dec-98 & $\mathrm{T} 155$ & 28 & 28.6 & 28.4 & ICP & ICP \\
\hline $\mathrm{Na}$ & $\mathrm{mg} / \mathrm{L}$ & Aug-99 & GWM4 & -- & 77.8 & 79.0 & na & ICP \\
\hline $\mathrm{NH}_{3}-\mathrm{N}$ & $\mathrm{mg} / \mathrm{L}$ & Jan-94 & N41p & -- & 1.22 & 1.22 & na & Colorimetry: phenate \\
\hline $\mathrm{NH}_{3}^{3}-\mathrm{N}$ & $\mathrm{mg} / \mathrm{L}$ & Feb-94 & $\mathrm{N} 40 \mathrm{p}$ & -- & 0.040 & 0.035 & na & Colorimetry: phenate \\
\hline $\mathrm{NH}_{3}^{3}-\mathrm{N}$ & $\mathrm{mg} / \mathrm{L}$ & Aug-94 & $\mathrm{N} 42 \mathrm{n}$ & $<0.2$ & -- & 0.25 & Colorimetry: phenate & na \\
\hline $\mathrm{NH}_{3}^{3}-\mathrm{N}$ & $\mathrm{mg} / \mathrm{L}$ & Aug-94 & $\mathrm{N} 42 \mathrm{p}$ & -- & 0.19 & 0.20 & na & Colorimetry: phenate \\
\hline $\mathrm{NH}_{3}^{3}-\mathrm{N}$ & $\mathrm{mg} / \mathrm{L}$ & Jan-95 & N43 & $<0.2$ & 0.110 & 0.11 & Colorimetry: phenate & Colorimetry: phenate \\
\hline $\mathrm{NH}_{3}^{3}-\mathrm{N}$ & $\mathrm{mg} / \mathrm{L}$ & Jan-95 & N44 & 0.9 & 0.760 & 0.900 & Colorimetry: phenate & Colorimetry: phenate \\
\hline $\mathrm{NH}_{3}-\mathrm{N}$ & $\mathrm{mg} / \mathrm{L}$ & Jul-95 & N45 & -- & 0.05 & 0.060 & na & Colorimetry: phenate \\
\hline $\mathrm{NH}_{3}-\mathrm{N}$ & $\mathrm{mg} / \mathrm{L}$ & Jul-95 & N46 & 1.0 & 1.08 & 1.04 & Colorimetry: phenate & Colorimetry: phenate \\
\hline $\mathrm{NH}_{3}^{3}-\mathrm{N}$ & $\mathrm{mg} / \mathrm{L}$ & Feb-96 & N47 & -- & -- & 0.165 & na & na \\
\hline $\mathrm{NH}_{3}^{3}-\mathrm{N}$ & $\mathrm{mg} / \mathrm{L}$ & Feb-96 & N48 & 0.6 & -- & 0.698 & Colorimetry: phenate & na \\
\hline $\mathrm{NH}_{3}^{3}-\mathrm{N}$ & $\mathrm{mg} / \mathrm{L}$ & Aug-96 & N49 & -- & 0.18 & 0.155 & na & Colorimetry: phenate \\
\hline $\mathrm{NH}_{3}^{3}-\mathrm{N}$ & $\mathrm{mg} / \mathrm{L}$ & Aug-96 & N50 & -- & 1.04 & 0.82 & na & Colorimetry: phenate \\
\hline $\mathrm{NH}_{3}-\mathrm{N}$ & $\mathrm{mg} / \mathrm{L}$ & Dec-98 & N59 & -- & 0.36 & 0.327 & na & Colorimetry: phenate \\
\hline $\mathrm{NH}_{3}-\mathrm{N}$ & $\mathrm{mg} / \mathrm{L}$ & Dec-98 & N60 & 0.5 & 0.64 & 0.578 & Colorimetry: phenate & Colorimetry: phenate \\
\hline $\mathrm{NH}_{3}^{3}-\mathrm{N}$ & $\mathrm{mg} / \mathrm{L}$ & Aug-99 & N61 & -- & 0.03 & 0.040 & na & Colorimetry: phenate \\
\hline $\mathrm{NH}_{3}-\mathrm{N}$ & $\mathrm{mg} / \mathrm{L}$ & Aug-99 & N62 & 0.8 & 1.04 & 1.01 & Colorimetry: indophenol & Colorimetry: phenate \\
\hline $\mathrm{NH}_{3}+$ Org N-N & $\mathrm{mg} / \mathrm{L}$ & Jan-94 & $\mathrm{N} 40 \mathrm{p}$ & -- & 0.07 & 0.270 & na & Colorimetry: phenate \\
\hline $\mathrm{NH}_{3}^{3}+$ Org N-N & $\mathrm{mg} / \mathrm{L}$ & Jan-94 & N41n & 1.6 & -- & 1.76 & Colorimetry: phenate & na \\
\hline $\mathrm{NH}_{3}^{3}+$ Org N-N & $\mathrm{mg} / \mathrm{L}$ & Jan-94 & N41p & -- & 1.67 & 1.86 & na & Colorimetry: phenate \\
\hline $\mathrm{NH}_{3}^{3}+$ Org N-N & $\mathrm{mg} / \mathrm{L}$ & Aug-94 & $\mathrm{N} 42 \mathrm{n}$ & 2.0 & -- & 1.70 & Colorimetry: phenate & na \\
\hline $\mathrm{NH}_{3}^{3}+$ Org N-N & $\mathrm{mg} / \mathrm{L}$ & Aug-94 & $\mathrm{N} 42 \mathrm{p}$ & -- & 1.70 & -- & na & Colorimetry: phenate \\
\hline $\mathrm{NH}_{3}^{3}+$ Org N-N & $\mathrm{mg} / \mathrm{L}$ & Jan-95 & N43 & $<0.3$ & 0.270 & 0.23 & Colorimetry: phenate & Colorimetry: phenate \\
\hline $\mathrm{NH}_{3}+$ Org N-N & $\mathrm{mg} / \mathrm{L}$ & Jan-95 & N44 & 1.0 & 1.21 & 1.24 & Colorimetry: phenate & Colorimetry: phenate \\
\hline $\mathrm{NH}_{3}^{3}+$ Org N-N & $\mathrm{mg} / \mathrm{L}$ & Jul-95 & N45 & -- & $<0.2$ & 0.300 & na & Colorimetry: phenate \\
\hline $\mathrm{NH}_{3}^{3}+$ Org N-N & $\mathrm{mg} / \mathrm{L}$ & Jul-95 & N46 & 2.4 & 1.68 & 1.81 & Colorimetry: phenate & Colorimetry: phenate \\
\hline $\mathrm{NH}_{3}^{3}+$ Org N-N & $\mathrm{mg} / \mathrm{L}$ & Feb-96 & N47 & 0.6 & -- & 0.48 & Colorimetry: phenate & na \\
\hline $\mathrm{NH}_{3}+$ Org N-N & $\mathrm{mg} / \mathrm{L}$ & Feb-96 & N48 & 1.6 & -- & 1.29 & Colorimetry: phenate & na \\
\hline $\mathrm{NH}_{3}^{3}+$ Org N-N & $\mathrm{mg} / \mathrm{L}$ & Aug-96 & N49 & -- & 0.38 & 0.33 & na & Colorimetry: phenate \\
\hline $\mathrm{NH}_{3}^{3}+$ Org N-N & $\mathrm{mg} / \mathrm{L}$ & Aug-96 & N50 & -- & 1.43 & 1.39 & na & Colorimetry: phenate \\
\hline
\end{tabular}


Appendix 8. Performance-evaluation data from the U.S. Geological Survey Standard Reference Program for the Metro Wastewater Reclamation District Laboratory (1993-1998) and the U.S. Geological Survey National Water Quality Laboratory (1993-1999).—Continued

[All abbreviations explained in appendix 2; USGS, U.S. Geological Survey; Metro District, Metro Wastewater Reclamation District Laboratory; NWQL, National Water Quality Laboratory; $\mu \mathrm{g} / \mathrm{L}$, micrograms per liter; $\mathrm{mg} / \mathrm{L}$, milligrams per liter; $\mu \mathrm{S} / \mathrm{cm}$, microsiemens per centimeter at 25 degrees Celsius; ICP, inductively coupled plasma; MS, mass spectrometry; --; no data; na, not applicable; AA, atomic absorption spectrometry; GFAA, graphite-furnace atomic absorption spectrometry; <, less than; DCP, direct current plasma; IC, ion chromatography]

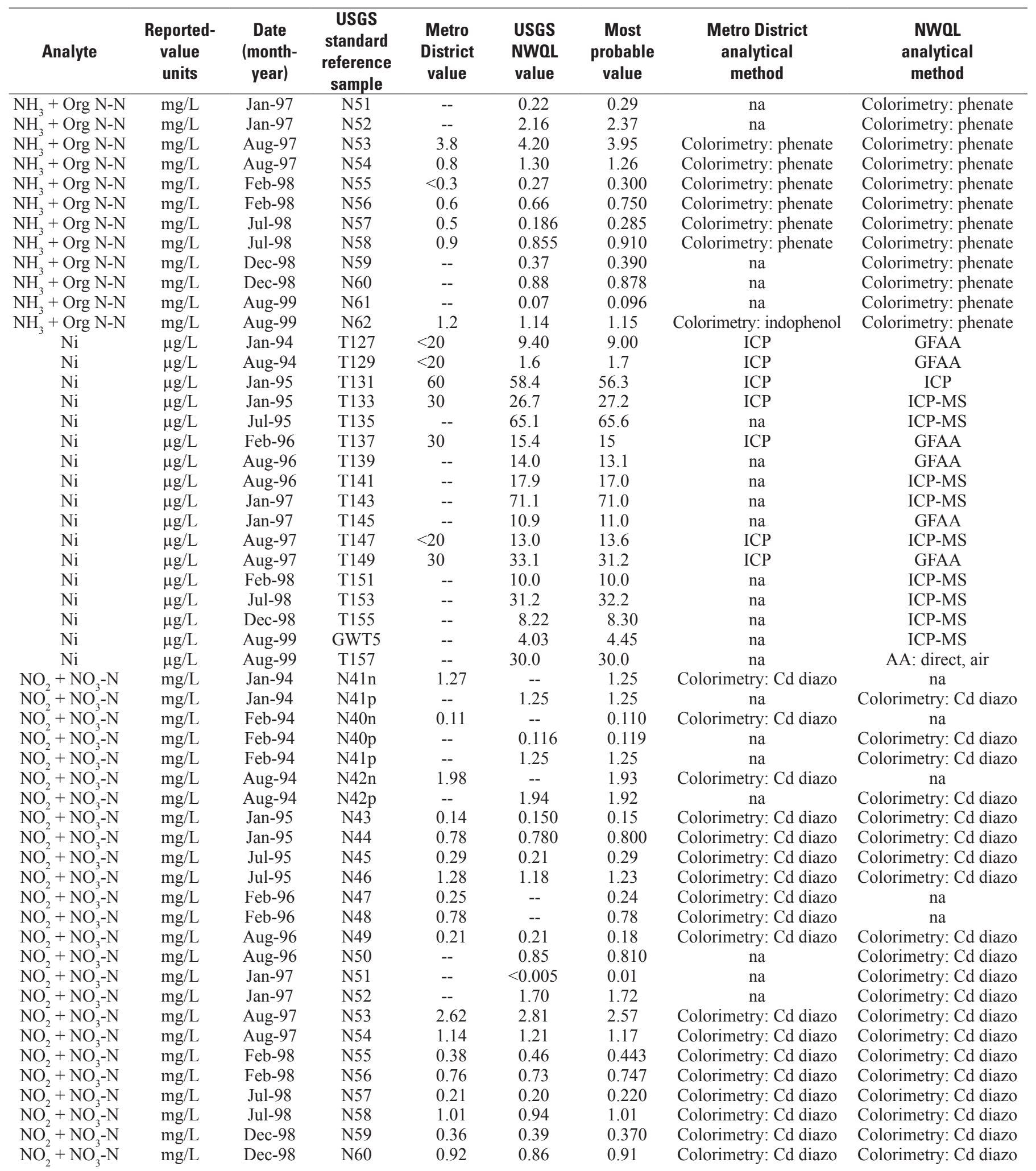


Appendix 8. Performance-evaluation data from the U.S. Geological Survey Standard Reference Program for the Metro Wastewater Reclamation District Laboratory (1993-1998) and the U.S. Geological Survey National Water Quality Laboratory (1993-1999).-Continued

[All abbreviations explained in appendix 2; USGS, U.S. Geological Survey; Metro District, Metro Wastewater Reclamation District Laboratory; NWQL, National Water Quality Laboratory; $\mu \mathrm{g} / \mathrm{L}$, micrograms per liter; $\mathrm{mg} / \mathrm{L}$, milligrams per liter; $\mu \mathrm{S} / \mathrm{cm}$, microsiemens per centimeter at 25 degrees Celsius; ICP, inductively coupled plasma; MS, mass spectrometry; --; no data; na, not applicable; AA, atomic absorption spectrometry; GFAA, graphite-furnace atomic absorption spectrometry; <, less than; DCP, direct current plasma; IC, ion chromatography]

\begin{tabular}{|c|c|c|c|c|c|c|c|c|}
\hline Analyte & $\begin{array}{l}\text { Reported- } \\
\text { value } \\
\text { units }\end{array}$ & $\begin{array}{c}\text { Date } \\
\text { (month- } \\
\text { year) }\end{array}$ & $\begin{array}{c}\text { USGS } \\
\text { standard } \\
\text { reference } \\
\text { sample }\end{array}$ & $\begin{array}{c}\text { Metro } \\
\text { District } \\
\text { value }\end{array}$ & $\begin{array}{l}\text { USGS } \\
\text { NWOL } \\
\text { value }\end{array}$ & $\begin{array}{c}\text { Most } \\
\text { probable } \\
\text { value }\end{array}$ & $\begin{array}{c}\text { Metro District } \\
\text { analytical } \\
\text { method }\end{array}$ & $\begin{array}{c}\text { NWOL } \\
\text { analytical } \\
\text { method }\end{array}$ \\
\hline $\mathrm{NO}_{2}+\mathrm{NO}_{3}-\mathrm{N}$ & $\mathrm{mg} / \mathrm{L}$ & Aug-99 & N61 & -- & 0.02 & 0.036 & na & Colorimetry: $\mathrm{Cd}$ diazo \\
\hline $\mathrm{NO}_{2}^{2}+\mathrm{NO}_{3}^{3}-\mathrm{N}$ & $\mathrm{mg} / \mathrm{L}$ & Aug-99 & N62 & 1.01 & 0.910 & 0.917 & na & Colorimetry: Cd diazo \\
\hline $\mathrm{P}^{3}$ & $\mathrm{mg} / \mathrm{L}$ & Jan-94 & M128 & 1.41 & 1.41 & 1.39 & $\begin{array}{c}\text { Colorimetry: } \\
\text { phosphomolybdate }\end{array}$ & $\begin{array}{l}\text { Colorimetry: } \\
\text { phosphomolybdate }\end{array}$ \\
\hline $\mathrm{P}$ & $\mathrm{mg} / \mathrm{L}$ & Jan-94 & N41n & 1.65 & -- & 1.63 & $\begin{array}{c}\text { Colorimetry: } \\
\text { phosphomolybdate }\end{array}$ & na \\
\hline $\mathrm{P}$ & $\mathrm{mg} / \mathrm{L}$ & Jan-94 & N41p & -- & 1.66 & 1.64 & na & $\begin{array}{c}\text { Colorimetry: } \\
\text { phosphomolybdate }\end{array}$ \\
\hline $\mathrm{P}$ & $\mathrm{mg} / \mathrm{L}$ & Feb-94 & $\mathrm{N} 40 \mathrm{p}$ & -- & 0.06 & 0.062 & na & $\begin{array}{l}\text { Colorimetry: } \\
\text { phosphomolybdate }\end{array}$ \\
\hline$P$ & $\mathrm{mg} / \mathrm{L}$ & Aug-94 & M130 & $<0.2$ & 0.006 & 0.085 & $\begin{array}{l}\text { Colorimetry: } \\
\text { phosphomolybdate }\end{array}$ & $\begin{array}{l}\text { Colorimetry: } \\
\text { phosphomolybdate }\end{array}$ \\
\hline$P$ & $\mathrm{mg} / \mathrm{L}$ & Aug-94 & $\mathrm{N} 42 \mathrm{n}$ & 1.13 & -- & 1.15 & $\begin{array}{c}\text { Colorimetry: } \\
\text { phosphomolybdate }\end{array}$ & na \\
\hline $\mathrm{P}$ & $\mathrm{mg} / \mathrm{L}$ & Aug-94 & $\mathrm{N} 42 \mathrm{p}$ & -- & 1.15 & 1.15 & na & $\begin{array}{c}\text { Colorimetry: } \\
\text { phosphomolybdate }\end{array}$ \\
\hline$P$ & $\mathrm{mg} / \mathrm{L}$ & Jan-95 & M132 & 0.03 & 0.06 & 0.03 & $\begin{array}{c}\text { Colorimetry: } \\
\text { phosphomolybdate }\end{array}$ & $\begin{array}{c}\text { Colorimetry: } \\
\text { phosphomolybdate }\end{array}$ \\
\hline $\mathrm{P}$ & $\mathrm{mg} / \mathrm{L}$ & Jan-95 & N43 & 0.14 & 0.120 & 0.13 & $\begin{array}{c}\text { Colorimetry: } \\
\text { phosphomolybdate }\end{array}$ & $\begin{array}{l}\text { Colorimetry: } \\
\text { phosphomolybdate }\end{array}$ \\
\hline$P$ & $\mathrm{mg} / \mathrm{L}$ & Jan-95 & N44 & 0.92 & 0.900 & 0.920 & $\begin{array}{c}\text { Colorimetry: } \\
\text { phosphomolybdate }\end{array}$ & $\begin{array}{c}\text { Colorimetry: } \\
\text { phosphomolybdate }\end{array}$ \\
\hline $\mathrm{P}$ & $\mathrm{mg} / \mathrm{L}$ & Jul-95 & N45 & 0.13 & 0.130 & 0.139 & $\begin{array}{c}\text { Colorimetry: } \\
\text { phosphomolybdate }\end{array}$ & $\begin{array}{c}\text { Colorimetry: } \\
\text { phosphomolybdate }\end{array}$ \\
\hline$P$ & $\mathrm{mg} / \mathrm{L}$ & Jul-95 & N46 & -- & 1.20 & 1.23 & na & $\begin{array}{c}\text { Colorimetry: } \\
\text { phosphomolybdate }\end{array}$ \\
\hline$P$ & $\mathrm{mg} / \mathrm{L}$ & Feb-96 & M136 & -- & 0.83 & 0.885 & 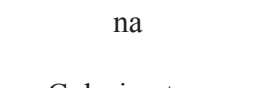 & $\begin{array}{l}\text { Colorimetry: } \\
\text { phosphomolybdate }\end{array}$ \\
\hline$P$ & $\mathrm{mg} / \mathrm{L}$ & Feb-96 & N47 & 0.17 & -- & 0.223 & $\begin{array}{c}\text { Colorimetry: } \\
\text { phosphomolybdate }\end{array}$ & na \\
\hline $\mathrm{P}$ & $\mathrm{mg} / \mathrm{L}$ & Feb-96 & N48 & 0.73 & -- & 0.794 & $\begin{array}{c}\text { Colorimetry: } \\
\text { phosphomolybdate }\end{array}$ & na \\
\hline$P$ & $\mathrm{mg} / \mathrm{L}$ & Apr-96 & M138 & 0.67 & 0.22 & 0.240 & $\begin{array}{l}\text { Colorimetry: } \\
\text { phosphomolybdate }\end{array}$ & $\begin{array}{c}\text { Colorimetry: } \\
\text { phosphomolybdate }\end{array}$ \\
\hline $\mathrm{P}$ & $\mathrm{mg} / \mathrm{L}$ & Aug-96 & N49 & 0.29 & 0.21 & 0.17 & $\begin{array}{c}\text { Colorimetry: } \\
\text { phosphomolybdate }\end{array}$ & $\begin{array}{l}\text { Colorimetry: } \\
\text { phosphomolybdate }\end{array}$ \\
\hline $\mathrm{P}$ & $\mathrm{mg} / \mathrm{L}$ & Aug-96 & N50 & -- & 1.03 & 0.903 & na & $\begin{array}{l}\text { Colorimetry: } \\
\text { phosphomolybdate }\end{array}$ \\
\hline$P$ & $\mathrm{mg} / \mathrm{L}$ & Jan-97 & M140 & -- & 0.030 & 0.032 & na & $\begin{array}{l}\text { Colorimetry: } \\
\text { phosphomolybdate }\end{array}$ \\
\hline $\mathrm{P}$ & $\mathrm{mg} / \mathrm{L}$ & Jan-97 & N51 & -- & 0.03 & 0.04 & na & $\begin{array}{l}\text { Colorimetry: } \\
\text { phosphomolybdate }\end{array}$ \\
\hline $\mathrm{P}$ & $\mathrm{mg} / \mathrm{L}$ & Jan-97 & N52 & -- & 1.55 & 1.60 & na & $\begin{array}{c}\text { Colorimetry: } \\
\text { phosphomolybdate }\end{array}$ \\
\hline$P$ & $\mathrm{mg} / \mathrm{L}$ & Aug-97 & M142 & $<0.02$ & 0.01 & 0.02 & $\begin{array}{c}\text { Colorimetry: } \\
\text { phosphomolybdate }\end{array}$ & $\begin{array}{l}\text { Colorimetry: } \\
\text { phosphomolybdate }\end{array}$ \\
\hline $\mathrm{P}$ & $\mathrm{mg} / \mathrm{L}$ & Aug-97 & N53 & 2.26 & 2.47 & 2.32 & $\begin{array}{c}\text { Colorimetry: } \\
\text { phosphomolybdate }\end{array}$ & $\begin{array}{l}\text { Colorimetry: } \\
\text { phosphomolybdate }\end{array}$ \\
\hline $\mathrm{P}$ & $\mathrm{mg} / \mathrm{L}$ & Aug-97 & N54 & 1.67 & 1.89 & 1.78 & $\begin{array}{c}\text { Colorimetry: } \\
\text { phosphomolybdate }\end{array}$ & $\begin{array}{l}\text { Colorimetry: } \\
\text { phosphomolybdate }\end{array}$ \\
\hline $\mathrm{P}$ & $\mathrm{mg} / \mathrm{L}$ & Feb-98 & GWM2 & 0.16 & 0.18 & 0.20 & $\begin{array}{l}\text { Colorimetry: } \\
\text { molybdate blue }\end{array}$ & $\begin{array}{c}\text { Colorimetry: } \\
\text { phosphomolybdate }\end{array}$ \\
\hline
\end{tabular}


Appendix 8. Performance-evaluation data from the U.S. Geological Survey Standard Reference Program for the Metro Wastewater Reclamation District Laboratory (1993-1998) and the U.S. Geological Survey National Water Quality Laboratory (1993-1999).-Continued

[All abbreviations explained in appendix 2; USGS, U.S. Geological Survey; Metro District, Metro Wastewater Reclamation District Laboratory; NWQL, National Water Quality Laboratory; $\mu \mathrm{g} / \mathrm{L}$, micrograms per liter; $\mathrm{mg} / \mathrm{L}$, milligrams per liter; $\mu \mathrm{S} / \mathrm{cm}$, microsiemens per centimeter at 25 degrees Celsius; ICP, inductively coupled plasma; MS, mass spectrometry; --; no data; na, not applicable; AA, atomic absorption spectrometry; GFAA, graphite-furnace atomic absorption spectrometry; <, less than; DCP, direct current plasma; IC, ion chromatography]

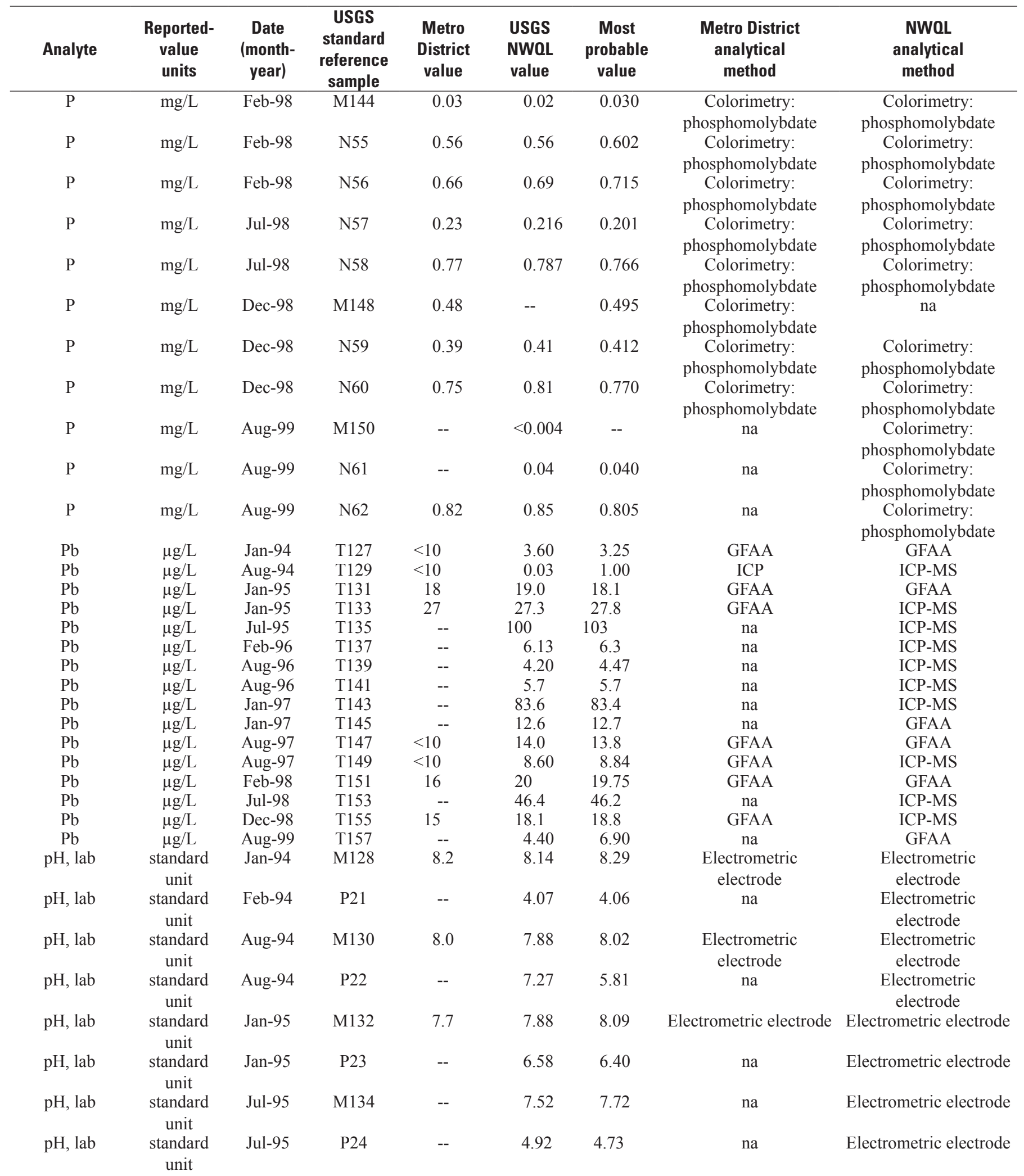


Appendix 8. Performance-evaluation data from the U.S. Geological Survey Standard Reference Program for the Metro Wastewater Reclamation District Laboratory (1993-1998) and the U.S. Geological Survey National Water Quality Laboratory (1993-1999).-Continued

[All abbreviations explained in appendix 2; USGS, U.S. Geological Survey; Metro District, Metro Wastewater Reclamation District Laboratory; NWQL, National Water Quality Laboratory; $\mu \mathrm{g} / \mathrm{L}$, micrograms per liter; $\mathrm{mg} / \mathrm{L}$, milligrams per liter; $\mu \mathrm{S} / \mathrm{cm}$, microsiemens per centimeter at 25 degrees Celsius; ICP, inductively coupled plasma; MS, mass spectrometry; --; no data; na, not applicable; AA, atomic absorption spectrometry; GFAA, graphite-furnace atomic absorption spectrometry; <, less than; DCP, direct current plasma; IC, ion chromatography]

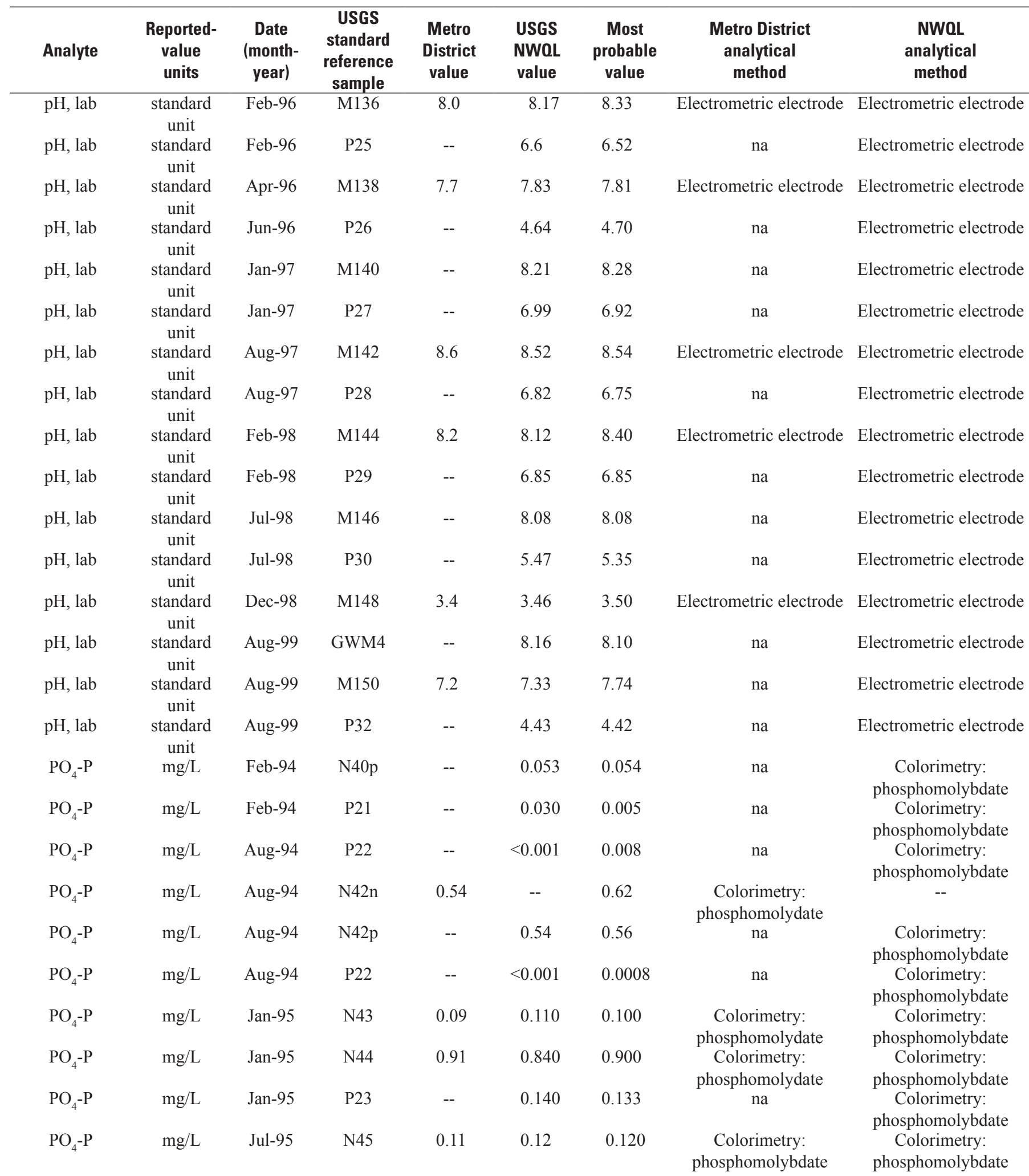


Appendix 8. Performance-evaluation data from the U.S. Geological Survey Standard Reference Program for the Metro Wastewater Reclamation District Laboratory (1993-1998) and the U.S. Geological Survey National Water Quality Laboratory (1993-1999).—Continued

[All abbreviations explained in appendix 2; USGS, U.S. Geological Survey; Metro District, Metro Wastewater Reclamation District Laboratory; NWQL, National Water Quality Laboratory; $\mu \mathrm{g} / \mathrm{L}$, micrograms per liter; $\mathrm{mg} / \mathrm{L}$, milligrams per liter; $\mu \mathrm{S} / \mathrm{cm}$, microsiemens per centimeter at 25 degrees Celsius; ICP, inductively coupled plasma; MS, mass spectrometry; --; no data; na, not applicable; AA, atomic absorption spectrometry; GFAA, graphite-furnace atomic absorption spectrometry; <, less than; DCP, direct current plasma; IC, ion chromatography]

\begin{tabular}{|c|c|c|c|c|c|c|c|c|}
\hline Analyte & $\begin{array}{l}\text { Reported- } \\
\text { value } \\
\text { units }\end{array}$ & $\begin{array}{c}\text { Date } \\
\text { (month- } \\
\text { year) }\end{array}$ & $\begin{array}{c}\text { USGS } \\
\text { standard } \\
\text { reference } \\
\text { sample }\end{array}$ & $\begin{array}{c}\text { Metro } \\
\text { District } \\
\text { value }\end{array}$ & $\begin{array}{l}\text { USGS } \\
\text { NWOL } \\
\text { value }\end{array}$ & $\begin{array}{c}\text { Most } \\
\text { probable } \\
\text { value }\end{array}$ & $\begin{array}{l}\text { Metro District } \\
\text { analytical } \\
\text { method }\end{array}$ & $\begin{array}{c}\text { NWQL } \\
\text { analytical } \\
\text { method }\end{array}$ \\
\hline $\mathrm{PO}_{4}-\mathrm{P}$ & $\mathrm{mg} / \mathrm{L}$ & Jul-95 & $\mathrm{P} 24$ & -- & 0.190 & 0.028 & na & IC \\
\hline $\mathrm{PO}_{4}^{4}-\mathrm{P}$ & $\mathrm{mg} / \mathrm{L}$ & Feb-96 & N47 & 0.13 & -- & 0.151 & $\begin{array}{c}\text { Colorimetry: } \\
\text { phosphomolybdate }\end{array}$ & na \\
\hline $\mathrm{PO}_{4}-\mathrm{P}$ & $\mathrm{mg} / \mathrm{L}$ & Feb-96 & P25 & -- & 0.06 & 0.068 & na & IC \\
\hline $\mathrm{PO}_{4}^{4}-\mathrm{P}$ & $\mathrm{mg} / \mathrm{L}$ & Jun-96 & $\mathrm{P} 26$ & -- & $<0.16$ & 0.004 & na & IC \\
\hline $\mathrm{PO}_{4}^{4}-\mathrm{P}$ & $\mathrm{mg} / \mathrm{L}$ & Aug-96 & N49 & 0.19 & 0.150 & 0.150 & $\begin{array}{c}\text { Colorimetry: } \\
\text { phosphomolybdate }\end{array}$ & $\begin{array}{c}\text { Colorimetry: } \\
\text { phosphomolybdate }\end{array}$ \\
\hline $\mathrm{PO}_{4}-\mathrm{P}$ & $\mathrm{mg} / \mathrm{L}$ & Aug-96 & N50 & -- & 0.92 & 0.73 & na & $\begin{array}{c}\text { Colorimetry: } \\
\text { phosphomolybdate }\end{array}$ \\
\hline $\mathrm{PO}_{4}-\mathrm{P}$ & $\mathrm{mg} / \mathrm{L}$ & Jan-97 & $\mathrm{P} 27$ & -- & $<0.001$ & 0.002 & na & $\begin{array}{l}\text { Colorimetry: } \\
\text { phosphomolybdate }\end{array}$ \\
\hline $\mathrm{PO}_{4}-\mathrm{P}$ & $\mathrm{mg} / \mathrm{L}$ & Aug-97 & N53 & 2.15 & 2.28 & 2.12 & $\begin{array}{c}\text { Colorimetry: } \\
\text { phosphomolybdate }\end{array}$ & $\begin{array}{l}\text { Colorimetry: } \\
\text { phosphomolybdate }\end{array}$ \\
\hline $\mathrm{PO}_{4}-\mathrm{P}$ & $\mathrm{mg} / \mathrm{L}$ & Aug-97 & N54 & 1.71 & 1.88 & 1.72 & $\begin{array}{c}\text { Colorimetry: } \\
\text { phosphomolybdate }\end{array}$ & $\begin{array}{c}\text { Colorimetry: } \\
\text { phosphomolybdate }\end{array}$ \\
\hline $\mathrm{PO}_{4}-\mathrm{P}$ & $\mathrm{mg} / \mathrm{L}$ & Feb-98 & N55 & 0.56 & 0.574 & 0.580 & $\begin{array}{c}\text { Colorimetry: } \\
\text { phosphomolybdate }\end{array}$ & $\begin{array}{l}\text { Colorimetry: } \\
\text { phosphomolybdate }\end{array}$ \\
\hline $\mathrm{PO}_{4}-\mathrm{P}$ & $\mathrm{mg} / \mathrm{L}$ & Feb-98 & N56 & 0.62 & 0.630 & 0.658 & $\begin{array}{c}\text { Colorimetry: } \\
\text { phosphomolybdate }\end{array}$ & $\begin{array}{c}\text { Colorimetry: } \\
\text { phosphomolybdate }\end{array}$ \\
\hline $\mathrm{PO}_{4}-\mathrm{P}$ & $\mathrm{mg} / \mathrm{L}$ & Feb-98 & P29 & -- & 0.04 & 0.046 & na & $\begin{array}{l}\text { Colorimetry: } \\
\text { phosphomolybdate }\end{array}$ \\
\hline $\mathrm{PO}_{4}-\mathrm{P}$ & $\mathrm{mg} / \mathrm{L}$ & Dec-98 & N60 & 0.65 & 0.641 & 0.680 & $\begin{array}{l}\text { Colorimetry: } \\
\text { phosphomolybdate }\end{array}$ & $\begin{array}{c}\text { Colorimetry: } \\
\text { phosphomolybdate }\end{array}$ \\
\hline $\mathrm{PO}_{4}-\mathrm{P}$ & $\mathrm{mg} / \mathrm{L}$ & Aug-99 & N61 & -- & 0.039 & 0.038 & na & $\begin{array}{l}\text { Colorimetry: } \\
\text { phosphomolybdate }\end{array}$ \\
\hline $\mathrm{PO}_{4}-\mathrm{P}$ & $\mathrm{mg} / \mathrm{L}$ & Aug-99 & N62 & 0.81 & 0.882 & 0.795 & na & $\begin{array}{l}\text { Colorimetry: } \\
\text { phosphomolybdate }\end{array}$ \\
\hline $\mathrm{PO}_{4}-\mathrm{P}$ & $\mathrm{mg} / \mathrm{L}$ & Aug-99 & P32 & -- & 0.180 & 0.177 & na & $\mathrm{IC}$ \\
\hline $\mathrm{Sb}$ & $\mu \mathrm{g} / \mathrm{L}$ & Feb-93 & $\mathrm{T} 121$ & $<100$ & 10.9 & 7.61 & -- & AA: hydride \\
\hline $\mathrm{Sb}$ & $\mu \mathrm{g} / \mathrm{L}$ & Aug-93 & T123 & -- & 7.18 & 6.99 & na & ICP/MS \\
\hline $\mathrm{Sb}$ & $\mu \mathrm{g} / \mathrm{L}$ & Aug-93 & $\mathrm{T} 125$ & -- & 6.05 & 6.24 & na & $\mathrm{ICP} / \mathrm{MS}$ \\
\hline $\mathrm{Sb}$ & $\mu \mathrm{g} / \mathrm{L}$ & Jan-94 & $\mathrm{T} 127$ & $<10$ & 5.10 & 5.15 & $\mathrm{ICP}$ & ICP-MS \\
\hline $\mathrm{Sb}$ & $\mu \mathrm{g} / \mathrm{L}$ & Aug-94 & T129 & $<100$ & 0.22 & 0.55 & $\mathrm{ICP}$ & ICP-MS \\
\hline $\mathrm{Sb}$ & $\mu \mathrm{g} / \mathrm{L}$ & Jan-95 & $\mathrm{T} 131$ & $<10$ & 57.4 & 56.2 & $\mathrm{ICP}$ & ICP-MS \\
\hline $\mathrm{Sb}$ & $\mu \mathrm{g} / \mathrm{L}$ & Jan-95 & T133 & $<10$ & 14.7 & 14.4 & ICP & ICP-MS \\
\hline $\mathrm{Sb}$ & $\mu \mathrm{g} / \mathrm{L}$ & Jul-95 & $\mathrm{T} 135$ & -- & 82.3 & 76.3 & na & GFAA \\
\hline $\mathrm{Sb}$ & $\mu \mathrm{g} / \mathrm{L}$ & Feb-96 & T137 & -- & 15.5 & 15.5 & na & GFAA \\
\hline
\end{tabular}


Appendix 8. Performance-evaluation data from the U.S. Geological Survey Standard Reference Program for the Metro Wastewater Reclamation District Laboratory (1993-1998) and the U.S. Geological Survey National Water Quality Laboratory (1993-1999).—Continued

[All abbreviations explained in appendix 2; USGS, U.S. Geological Survey; Metro District, Metro Wastewater Reclamation District Laboratory; NWQL, National Water Quality Laboratory; $\mu \mathrm{g} / \mathrm{L}$, micrograms per liter; $\mathrm{mg} / \mathrm{L}$, milligrams per liter; $\mu \mathrm{S} / \mathrm{cm}$, microsiemens per centimeter at 25 degrees Celsius; ICP, inductively coupled plasma; MS, mass spectrometry; --; no data; na, not applicable; AA, atomic absorption spectrometry; GFAA, graphite-furnace atomic absorption spectrometry; <, less than; DCP, direct current plasma; IC, ion chromatography]

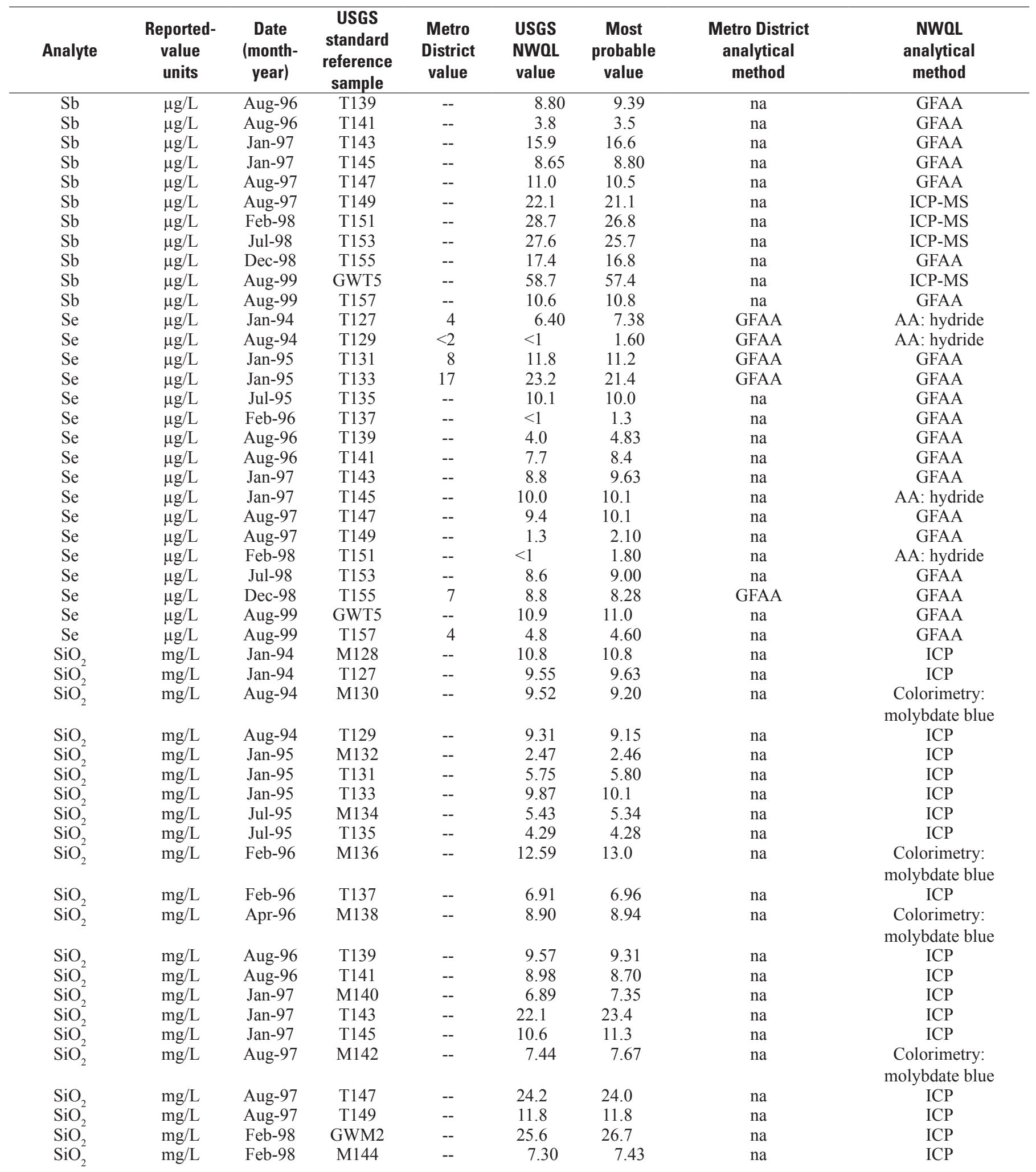


Appendix 8. Performance-evaluation data from the U.S. Geological Survey Standard Reference Program for the Metro Wastewater Reclamation District Laboratory (1993-1998) and the U.S. Geological Survey National Water Quality Laboratory (1993-1999).-Continued

[All abbreviations explained in appendix 2; USGS, U.S. Geological Survey; Metro District, Metro Wastewater Reclamation District Laboratory; NWQL, National Water Quality Laboratory; $\mu \mathrm{g} / \mathrm{L}$, micrograms per liter; $\mathrm{mg} / \mathrm{L}$, milligrams per liter; $\mu \mathrm{S} / \mathrm{cm}$, microsiemens per centimeter at 25 degrees Celsius; ICP, inductively coupled plasma; MS, mass spectrometry; --; no data; na, not applicable; AA, atomic absorption spectrometry; GFAA, graphite-furnace atomic absorption spectrometry; <, less than; DCP, direct current plasma; IC, ion chromatography]

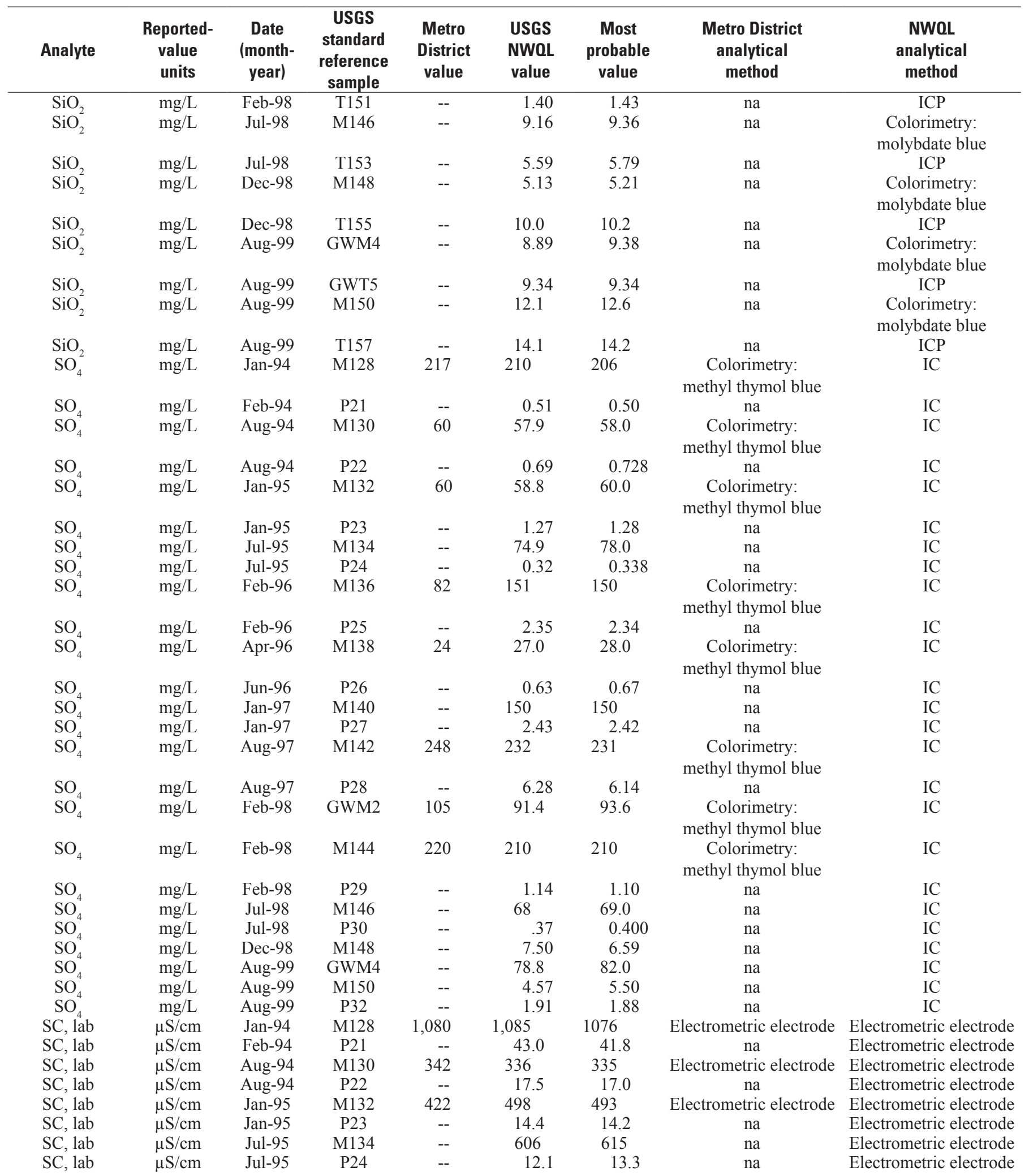


Appendix 8. Performance-evaluation data from the U.S. Geological Survey Standard Reference Program for the Metro Wastewater Reclamation District Laboratory (1993-1998) and the U.S. Geological Survey National Water Quality Laboratory (1993-1999).—Continued

[All abbreviations explained in appendix 2; USGS, U.S. Geological Survey; Metro District, Metro Wastewater Reclamation District Laboratory; NWQL, National Water Quality Laboratory; $\mu \mathrm{g} / \mathrm{L}$, micrograms per liter; $\mathrm{mg} / \mathrm{L}$, milligrams per liter; $\mu \mathrm{S} / \mathrm{cm}$, microsiemens per centimeter at 25 degrees Celsius; ICP, inductively coupled plasma; MS, mass spectrometry; --; no data; na, not applicable; AA, atomic absorption spectrometry; GFAA, graphite-furnace atomic absorption spectrometry; <, less than; DCP, direct current plasma; IC, ion chromatography]

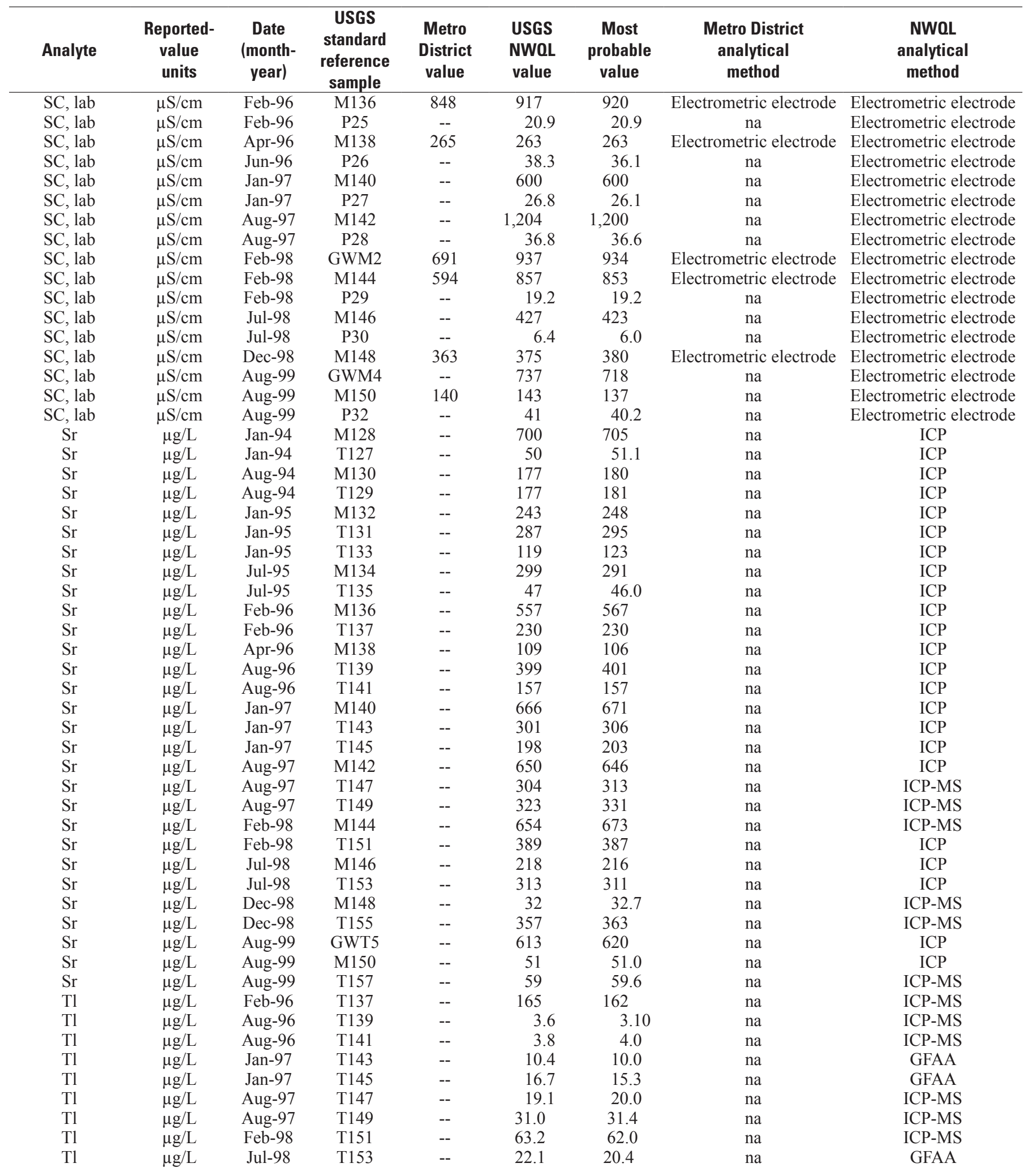


Appendix 8. Performance-evaluation data from the U.S. Geological Survey Standard Reference Program for the Metro Wastewater Reclamation District Laboratory (1993-1998) and the U.S. Geological Survey National Water Quality Laboratory (1993-1999).-Continued

[All abbreviations explained in appendix 2; USGS, U.S. Geological Survey; Metro District, Metro Wastewater Reclamation District Laboratory; NWQL, National Water Quality Laboratory; $\mu \mathrm{g} / \mathrm{L}$, micrograms per liter; $\mathrm{mg} / \mathrm{L}$, milligrams per liter; $\mu \mathrm{S} / \mathrm{cm}$, microsiemens per centimeter at 25 degrees Celsius; ICP, inductively coupled plasma; MS, mass spectrometry; --; no data; na, not applicable; AA, atomic absorption spectrometry; GFAA, graphite-furnace atomic absorption spectrometry; <, less than; DCP, direct current plasma; IC, ion chromatography]

\begin{tabular}{|c|c|c|c|c|c|c|c|c|}
\hline Analyte & $\begin{array}{l}\text { Reported- } \\
\text { value } \\
\text { units }\end{array}$ & $\begin{array}{c}\text { Date } \\
\text { (month- } \\
\text { year) }\end{array}$ & $\begin{array}{c}\text { USGS } \\
\text { standard } \\
\text { reference } \\
\text { sample } \\
\end{array}$ & $\begin{array}{c}\text { Metro } \\
\text { District } \\
\text { value }\end{array}$ & $\begin{array}{l}\text { USGS } \\
\text { NWQL } \\
\text { value }\end{array}$ & $\begin{array}{c}\text { Most } \\
\text { probable } \\
\text { value }\end{array}$ & $\begin{array}{l}\text { Metro District } \\
\text { analytical } \\
\text { method }\end{array}$ & $\begin{array}{c}\text { NWQL } \\
\text { analytical } \\
\text { method }\end{array}$ \\
\hline $\mathrm{Tl}$ & $\mu \mathrm{g} / \mathrm{L}$ & Aug-99 & $\mathrm{T} 157$ & -- & 9.4 & 8.75 & na & GFAA \\
\hline $\mathrm{U}$ & $\mu \mathrm{g} / \mathrm{L}$ & Aug-96 & T139 & -- & 4.8 & 5.00 & na & ICP-MS \\
\hline $\mathrm{U}$ & $\mu \mathrm{g} / \mathrm{L}$ & Aug-96 & $\mathrm{T} 141$ & -- & 3.7 & 3.85 & na & ICP-MS \\
\hline $\mathrm{U}$ & $\mu \mathrm{g} / \mathrm{L}$ & Aug-97 & $\mathrm{T} 147$ & -- & 3.0 & 3.21 & na & ICP-MS \\
\hline $\mathrm{U}$ & $\mu \mathrm{g} / \mathrm{L}$ & Aug-97 & T149 & -- & 2.4 & 2.71 & na & ICP-MS \\
\hline $\mathrm{U}$ & $\mu \mathrm{g} / \mathrm{L}$ & Feb-98 & $\mathrm{T} 151$ & -- & 15.0 & 15.0 & na & ICP-MS \\
\hline $\mathrm{U}$ & $\mu \mathrm{g} / \mathrm{L}$ & Jul-98 & $\mathrm{T} 153$ & -- & 7.1 & 6.90 & na & ICP-MS \\
\hline $\mathrm{U}$ & $\mu \mathrm{g} / \mathrm{L}$ & Dec-98 & $\mathrm{T} 155$ & -- & 7.0 & 7.50 & na & ICP-MS \\
\hline $\mathrm{U}$ & $\mu \mathrm{g} / \mathrm{L}$ & Aug-99 & GWT5 & -- & 6.4 & 6.42 & na & ICP-MS \\
\hline V & $\mu \mathrm{g} / \mathrm{L}$ & Jan-95 & $\mathrm{T} 131$ & -- & 31.7 & 34.2 & na & ICP \\
\hline $\mathrm{V}$ & $\mu \mathrm{g} / \mathrm{L}$ & Jan-95 & $\mathrm{T} 133$ & -- & 11.9 & 13.0 & na & ICP \\
\hline V & $\mu \mathrm{g} / \mathrm{L}$ & Jul-95 & M134 & -- & 2.73 & 3.55 & na & Colorimetry \\
\hline $\mathrm{V}$ & $\mu \mathrm{g} / \mathrm{L}$ & Jul-95 & $\mathrm{T} 135$ & -- & 52.9 & 52.8 & na & ICP \\
\hline $\mathrm{V}$ & $\mu \mathrm{g} / \mathrm{L}$ & Feb-96 & M136 & -- & 5.98 & 6.9 & na & Colorimetry \\
\hline $\mathrm{V}$ & $\mu \mathrm{g} / \mathrm{L}$ & Feb-96 & $\mathrm{T} 137$ & -- & 13.3 & 14.0 & na & $\mathrm{ICP}$ \\
\hline $\mathrm{V}$ & $\mu \mathrm{g} / \mathrm{L}$ & Apr-96 & M138 & -- & 19.7 & 16.5 & na & Colorimetry \\
\hline $\mathrm{V}$ & $\mu \mathrm{g} / \mathrm{L}$ & Aug-96 & T139 & -- & 4.3 & 5.0 & na & ICP \\
\hline V & $\mu \mathrm{g} / \mathrm{L}$ & Aug-96 & T141 & -- & 9.30 & 9.45 & na & ICP \\
\hline $\mathrm{V}$ & $\mu \mathrm{g} / \mathrm{L}$ & Jan-97 & M140 & -- & 1.40 & 3.42 & na & Colorimetry \\
\hline $\mathrm{V}$ & $\mu \mathrm{g} / \mathrm{L}$ & Jan-97 & $\mathrm{T} 143$ & -- & 31.1 & 30.0 & na & ICP \\
\hline $\mathrm{V}$ & $\mu \mathrm{g} / \mathrm{L}$ & Jan-97 & $\mathrm{T} 145$ & -- & 12.0 & 11.7 & na & $\mathrm{ICP}$ \\
\hline $\mathrm{V}$ & $\mu \mathrm{g} / \mathrm{L}$ & Aug-99 & $\mathrm{T} 157$ & -- & 17.1 & 15.7 & na & ICP-MS \\
\hline $\mathrm{Zn}$ & $\mu \mathrm{g} / \mathrm{L}$ & Jan-94 & $\mathrm{T} 127$ & 30 & 31.8 & 32.9 & ICP & ICP-MS \\
\hline $\mathrm{Zn}$ & $\mu \mathrm{g} / \mathrm{L}$ & Aug-94 & T129 & 70 & 67.7 & 72.0 & ICP & ICP-MS \\
\hline $\mathrm{Zn}$ & $\mu \mathrm{g} / \mathrm{L}$ & Jan-95 & $\mathrm{T} 131$ & 80 & 74.4 & 72.0 & $\mathrm{ICP}$ & ICP \\
\hline $\mathrm{Zn}$ & $\mu \mathrm{g} / \mathrm{L}$ & Jan-95 & $\mathrm{T} 133$ & 50 & 51.5 & 53.0 & $\mathrm{ICP}$ & ICP-MS \\
\hline $\mathrm{Zn}$ & $\mu \mathrm{g} / \mathrm{L}$ & Jul-95 & $\mathrm{T} 135$ & -- & 65.3 & 48.2 & na & ICP-MS \\
\hline $\mathrm{Zn}$ & $\mu \mathrm{g} / \mathrm{L}$ & Feb-96 & T137 & 50 & 45.9 & 49.5 & ICP & ICP-MS \\
\hline $\mathrm{Zn}$ & $\mu \mathrm{g} / \mathrm{L}$ & Aug-96 & T139 & 60 & 7.9 & 11 & ICP & ICP-MS \\
\hline $\mathrm{Zn}$ & $\mu \mathrm{g} / \mathrm{L}$ & Aug-96 & T141 & 220 & 216 & 218 & ICP & ICP \\
\hline $\mathrm{Zn}$ & $\mu \mathrm{g} / \mathrm{L}$ & Jan-97 & T143 & -- & 18.7 & 20.0 & na & ICP-MS \\
\hline $\mathrm{Zn}$ & $\mu \mathrm{g} / \mathrm{L}$ & Jan-97 & T145 & -- & 11.4 & 10.0 & na & ICP-MS \\
\hline $\mathrm{Zn}$ & $\mu \mathrm{g} / \mathrm{L}$ & Aug-97 & T147 & $<20$ & 12.6 & 14.0 & ICP & ICP-MS \\
\hline $\mathrm{Zn}$ & $\mu \mathrm{g} / \mathrm{L}$ & Aug-97 & T149 & $<20$ & 4.90 & 5.80 & ICP & ICP-MS \\
\hline $\mathrm{Zn}$ & $\mu \mathrm{g} / \mathrm{L}$ & Feb-98 & $\mathrm{T} 151$ & -- & 6.30 & 6.57 & na & ICP-MS \\
\hline $\mathrm{Zn}$ & $\mu \mathrm{g} / \mathrm{L}$ & Jul-98 & $\mathrm{T} 153$ & -- & 78.1 & 72.6 & na & ICP \\
\hline $\mathrm{Zn}$ & $\mu \mathrm{g} / \mathrm{L}$ & Dec-98 & $\mathrm{T} 155$ & -- & 55.9 & 58.7 & na & ICP-MS \\
\hline $\mathrm{Zn}$ & $\mu \mathrm{g} / \mathrm{L}$ & Aug-99 & GWT5 & 19 & 15.1 & 15.9 & GFAA & ICP-MS \\
\hline $\mathrm{Zn}$ & $\mu \mathrm{g} / \mathrm{L}$ & Aug-99 & $\mathrm{T} 157$ & 34 & 23.5 & 23.5 & GFAA & ICP-MS \\
\hline
\end{tabular}


Appendix 9. Geochemical modeling results for well D6 using NETPATH for groundwater samples collected near Deer Trail, Colorado, 1993 to 1999.

[Mont, montmorillonite; K-spar, orthoclase; “CH2O,” organic matter, such as biosolids; EX, ion exchange]

Saturation of major minerals in well D6 groundwater calculated from water-quality data by using the NETPATH model (Plummer and others, 1994):

\begin{tabular}{llcl}
\hline \multicolumn{1}{c}{ Mineral name } & Mineral formula & Saturation index & \multicolumn{1}{c}{ Mineral status } \\
\hline Anhydrite & $\mathrm{CaSO}_{4}$ & -0.05 & Stable, or precipitating slightly \\
Gypsum & $\mathrm{CaSO}_{4} \cdot 7 \mathrm{H}_{2} \mathrm{O}$ & 0.19 & Dissolving \\
Calcite & $\mathrm{CaCO}_{3}$ & 0.21 & Dissolving \\
Dolomite & $(\mathrm{Ca}, \mathrm{Mg})\left(\mathrm{CO}_{3}\right)_{2}$ & 1.20 & Dissolving \\
Quartz & $\mathrm{SiO}_{2}$ & 0.90 & Dissolving \\
Chalcedony & $\mathrm{SiO}_{2}$ & 0.42 & Dissolving \\
Amorphous $\mathrm{SiO}_{2}$ & $\mathrm{SiO}_{2}$ & -0.46 & Precipitating \\
\hline
\end{tabular}

Simulation 1 to determine if groundwater from well D6 evolved from upgradient wells D5, D10, D29, or D32

Initial Well 1: D29

Initial Well 2: D5

Initial Well 3: D10

Initial Well 4: D32

Final well: D6

\begin{tabular}{|c|c|c|c|c|c|c|c|c|c|c|c|c|}
\hline Constituent & Final well & $\begin{array}{l}\text { Initial } \\
\text { well } 1 \\
\end{array}$ & $\begin{array}{l}\text { Initial } \\
\text { well } 2 \\
\end{array}$ & $\begin{array}{l}\text { Initial } \\
\text { well } 3\end{array}$ & $\begin{array}{l}\text { Initial } \\
\text { well } 4 \\
\end{array}$ & & & & & & & \\
\hline $\mathrm{C}$ & 11.0964 & 5.9986 & 4.1627 & 8.3823 & 5.8406 & & & & & & & \\
\hline S & 137.0132 & 31.3221 & 25.7201 & 34.9691 & 24.3758 & & & & & & & \\
\hline $\mathrm{Ca}$ & 13.6586 & 16.2698 & 12.4074 & 14.3953 & 14.8926 & & & & & & & \\
\hline $\mathrm{Mg}$ & 92.4959 & 14.1358 & 11.4155 & 17.7619 & 9.5967 & & & & & & & \\
\hline $\mathrm{Na}$ & 82.3245 & 6.0903 & 8.8408 & 13.9122 & 6.1737 & & & & & & & \\
\hline $\mathrm{Si}$ & 0.4579 & 0.3578 & 0 & 0 & 0 & & & & & & & \\
\hline $\mathrm{Mn}$ & 0.0893 & 0.0148 & 0 & 0 & 0 & & & & & & & \\
\hline $\mathrm{Fe}$ & 0 & 0.1234 & 0 & 0 & 0 & & & & & & & \\
\hline Aragonite & $\mathrm{Ca}$ & 1 & $\mathrm{C}$ & 1 & RS & 4 & I1 & 0 & I2 & 0 & & \\
\hline Exchange & $\mathrm{Ca}$ & -1 & $\mathrm{Na}$ & 2 & $\mathrm{Mg}$ & 0 & & & & & & \\
\hline Lignite & $\mathrm{C}$ & 1 & RS & -0.4 & I1 & -25 & I2 & 0 & & & & \\
\hline Pyrite & $\mathrm{Fe}$ & 1 & $\mathrm{~S}$ & 2 & RS & 0 & I3 & -60 & & & & \\
\hline Calcite & $\mathrm{Ca}$ & 1 & $\mathrm{C}$ & 1 & RS & 4 & I1 & 0 & I2 & 0 & & \\
\hline Gypsum & $\mathrm{Ca}$ & 1 & $\mathrm{~S}$ & 1 & RS & 6 & I3 & 22 & & & & \\
\hline "CH2O" & $\mathrm{C}$ & 1 & I1 & -25 & I2 & 0 & & & & & & \\
\hline Dolomite & $\mathrm{Ca}$ & 1 & $\mathrm{Mg}$ & 1 & $\mathrm{C}$ & 2 & $\mathrm{RS}$ & 8 & I1 & 0 & I2 & 0 \\
\hline $\mathrm{CO} 2$ gas & $\mathrm{C}$ & 1 & $\mathrm{RS}$ & 4 & I1 & -25 & I2 & 100 & & & & \\
\hline Goethite & $\mathrm{Fe}$ & 1 & RS & 3 & & & & & & & & \\
\hline Anorthosite & $\mathrm{Ca}$ & 1 & $\mathrm{Al}$ & 2 & $\mathrm{Si}$ & 2 & & & & & & \\
\hline Barite & $\mathrm{Ba}$ & 1 & $\mathrm{~S}$ & 1 & RS & 6 & & & & & & \\
\hline $\mathrm{Mn}(\mathrm{OH}) 3$ & $\mathrm{Mn}$ & 1 & RS & 3 & & & & & & & & \\
\hline Albite & $\mathrm{Na}$ & 1 & $\mathrm{Al}$ & 1 & $\mathrm{Si}$ & 3 & & & & & & \\
\hline K-Spar & $\mathrm{K}$ & 1 & $\mathrm{Al}$ & 1 & $\mathrm{Si}$ & 3 & & & & & & \\
\hline Kaolinite & $\mathrm{Al}$ & 2 & $\mathrm{Si}$ & 2 & & & & & & & & \\
\hline Ca-Mont & $\mathrm{Ca}$ & 0.167 & $\mathrm{Al}$ & 2.33 & $\mathrm{Si}$ & 3.67 & & & & & & \\
\hline Na-Mont & $\mathrm{Na}$ & 0.33 & $\mathrm{Al}$ & 2.33 & $\mathrm{Si}$ & 3.67 & & & & & & \\
\hline $\mathrm{SiO} 2$ & $\mathrm{Si}$ & 1 & & & & & & & & & & \\
\hline 0 & models found & & & & & & & & & & & \\
\hline
\end{tabular}


Appendix 9. Geochemical modeling results for well D6 using NETPATH for groundwater samples collected near Deer Trail, Colorado, 1993 to 1999. - Continued

Simulation 2 to determine if groundwater from well D6 evolved from pure rainwater

Initial well: $\quad$ pure rainwater

Final well: D6

\begin{tabular}{|c|c|c|c|c|c|c|c|c|c|c|c|c|}
\hline Constituent & Final well & Initial well & & & & & & & & & & \\
\hline S & 137.0132 & 0 & & & & & & & & & & \\
\hline $\mathrm{Ca}$ & 13.6586 & 0 & & & & & & & & & & \\
\hline $\mathrm{Mg}$ & 92.4959 & 0 & & & & & & & & & & \\
\hline $\mathrm{Na}$ & 82.3245 & 0 & & & & & & & & & & \\
\hline $\mathrm{Si}$ & 0.4579 & 0 & & & & & & & & & & \\
\hline Gypsum & $\mathrm{Ca}$ & 1 & $\mathrm{~S}$ & 1 & $\mathrm{RS}$ & 6 & $\mathrm{I} 3$ & 22 & & & & \\
\hline $\mathrm{Mg} / \mathrm{Na} \mathrm{EX}$ & $\mathrm{Na}$ & 2 & $\mathrm{Mg}$ & -1 & & & & & & & & \\
\hline Calcite & $\mathrm{Ca}$ & 1 & $\mathrm{C}$ & 1 & $\mathrm{RS}$ & 4 & I1 & 0 & $\mathrm{I} 2$ & 0 & & \\
\hline "CH2O" & $\mathrm{C}$ & 1 & I1 & -25 & $\mathrm{I} 2$ & 0 & & & & & & \\
\hline Kaolinite & $\mathrm{Al}$ & 2 & $\mathrm{Si}$ & 2 & & & & & & & & \\
\hline Ca-Mont & $\mathrm{Ca}$ & 0.167 & $\mathrm{Al}$ & 2.33 & $\mathrm{Si}$ & 3.67 & & & & & & \\
\hline Na-Mont & $\mathrm{Na}$ & 0.33 & $\mathrm{Al}$ & 2.33 & $\mathrm{Si}$ & 3.67 & & & & & & \\
\hline
\end{tabular}

\begin{tabular}{|lcc|}
\hline & Model 1 & \\
Gypsum & $+\mathrm{F}$ & 137.0132 \\
Dolomite & $+\mathrm{F}$ & 5.54818 \\
$\mathrm{NaCl}$ & $+\mathrm{F}$ & 256.21995 \\
$\mathrm{Mg} / \mathrm{Na} \mathrm{EX}$ & & -86.94775 \\
Kaolinite & & 1416.61598 \\
Ca-Mont & & -771.87305 \\
\hline
\end{tabular}

\begin{tabular}{|lcr|}
\hline & Model 2 & \\
Gypsum & $+\mathrm{F}$ & 137.0132 \\
Dolomite & $+\mathrm{F}$ & 5.54818 \\
$\mathrm{NaCl}$ & $+\mathrm{F}$ & 1.46067 \\
$\mathrm{Mg} / \mathrm{Na} \mathrm{EX}$ & & -86.94775 \\
$\mathrm{Ca}-$ Mont & & -771.87305 \\
$\mathrm{Na}-$ Mont & & 771.99781 \\
\hline
\end{tabular}

\begin{tabular}{|lcr|}
\hline & Model 3 & \\
Gypsum & $+\mathrm{F}$ & 137.0132 \\
Dolomite & $+\mathrm{F}$ & 92.49593 \\
$\mathrm{NaCl}$ & $+\mathrm{F}$ & 82.32445 \\
Calcite & & -215.85055 \\
"CH2O" & + & 41.95505 \\
Kaolinite & & 0.22893 \\
\hline
\end{tabular}

\begin{tabular}{|lcr|}
\hline & Model 4 & \\
Gypsum & $+\mathrm{F}$ & 137.0132 \\
Dolomite & $+\mathrm{F}$ & 92.49593 \\
$\mathrm{NaCl}$ & $+\mathrm{F}$ & 82.32445 \\
Calcite & & -215.87138 \\
"CH2O" & + & 41.97588 \\
Ca-Mont & & 0.12476 \\
\hline
\end{tabular}

\begin{tabular}{|lcr|}
\hline & Model 5 & \\
Gypsum & $+\mathrm{F}$ & 137.0132 \\
Dolomite & $+\mathrm{F}$ & 92.49593 \\
$\mathrm{NaCl}$ & $+\mathrm{F}$ & 82.28328 \\
Calcite & & -215.85055 \\
"CH2O" & + & 41.95505 \\
Na-Mont & & 0.12476 \\
\hline
\end{tabular}

\begin{tabular}{|lcr|}
\hline & Model 6 & \\
Gypsum & $+\mathrm{F}$ & 137.0132 \\
Dolomite & $+\mathrm{F}$ & 92.03807 \\
$\mathrm{NaCl}$ & $+\mathrm{F}$ & 82.32445 \\
$\mathrm{Calcite}$ & & -215.39269 \\
"CH2O" & + & 42.41291 \\
$\mathrm{MgSiO} 3$ & + & 0.45786 \\
\hline
\end{tabular}

\begin{tabular}{|lcc|}
\hline & Model 7 & \\
Gypsum & $+\mathrm{F}$ & 137.0132 \\
Dolomite & $+\mathrm{F}$ & 92.49593 \\
$\mathrm{NaCl}$ & $+\mathrm{F}$ & 82.32445 \\
Calcite & & -173.8955 \\
Kaolinite & & 461.23202 \\
Ca-Mont & & -251.22784 \\
\hline
\end{tabular}

\begin{tabular}{|lcr|}
\hline & Model 8 & \\
Gypsum & $+\mathrm{F}$ & 137.0132 \\
Dolomite & $+\mathrm{F}$ & 92.49593 \\
$\mathrm{NaCl}$ & $+\mathrm{F}$ & 82.32445 \\
Calcite & & -215.85055 \\
Kaolinite & & 0.22893 \\
Lignite & + & 41.95505 \\
\hline
\end{tabular}

\begin{tabular}{|lcr|}
\hline & Model 9 & \\
Gypsum & $+\mathrm{F}$ & 137.0132 \\
Dolomite & $+\mathrm{F}$ & 92.49593 \\
$\mathrm{NaCl}$ & $+\mathrm{F}$ & 82.32445 \\
Calcite & & -215.87138 \\
Ca-Mont & & 0.12476 \\
Lignite & + & 41.97588 \\
\hline
\end{tabular}

\begin{tabular}{|lrr|}
\hline & Model 10 & \\
Gypsum & $+\mathrm{F}$ & 137.0132 \\
Dolomite & $+\mathrm{F}$ & 92.49593 \\
$\mathrm{NaCl}$ & $+\mathrm{F}$ & 82.28328 \\
Calcite & & -215.85055 \\
Na-Mont & & 0.12476 \\
Lignite & + & 41.95505 \\
\hline
\end{tabular}

\begin{tabular}{|lcr|}
\hline & Model 11 & \\
Gypsum & $+\mathrm{F}$ & 137.0132 \\
Dolomite & $+\mathrm{F}$ & 92.03807 \\
$\mathrm{NaCl}$ & $+\mathrm{F}$ & 82.32445 \\
$\mathrm{Calcite}$ & & -215.39269 \\
MgSiO3 & + & 0.45786 \\
Lignite & + & 42.41291 \\
\hline
\end{tabular}

\begin{tabular}{|lcr|}
\hline & $\begin{array}{c}\text { Model 12 } \\
\text { Gypsum }\end{array}$ & \\
Dolomite & $+\mathrm{F}$ & 137.0132 \\
$\mathrm{NaCl}$ & $+\mathrm{F}$ & 5.54818 \\
Kaolinite & & 82.32445 \\
Ca-Mont & & 1373.14211 \\
MgSiO3 & + & -771.87305 \\
\hline
\end{tabular}


Appendix 9. Geochemical modeling results for well D6 using NETPATH for groundwater samples collected near Deer Trail, Colorado, 1993 to 1999.-Continued

Simulation 3 to determine if groundwater from well D6 evolved from pure rainwater

Initial Well : pure rainwater

Final Well : D6

\begin{tabular}{|c|c|c|c|c|c|c|c|c|c|c|c|c|}
\hline Constituent & Final well & Initial well & & & & & & & & & & \\
\hline $\mathrm{C}$ & 11.0964 & 0 & & & & & & & & & & \\
\hline $\mathrm{S}$ & 137.0132 & 0 & & & & & & & & & & \\
\hline $\mathrm{Ca}$ & 13.6586 & 0 & & & & & & & & & & \\
\hline $\mathrm{Mg}$ & 92.4959 & 0 & & & & & & & & & & \\
\hline $\mathrm{Na}$ & 82.3245 & 0 & & & & & & & & & & \\
\hline $\mathrm{Si}$ & 0.4579 & 0 & & & & & & & & & & \\
\hline $\mathrm{Cl}$ & 10.6481 & 0 & & & & & & & & & & \\
\hline Gypsum & $\mathrm{Ca}$ & 1 & $\mathrm{~S}$ & 1 & RS & 6 & $\mathrm{I} 3$ & 22 & & & & \\
\hline Dolomite & $\mathrm{Ca}$ & 1 & $\mathrm{Mg}$ & 1 & $\mathrm{C}$ & 2 & RS & 8 & I1 & 0 & $\mathrm{I} 2$ & 0 \\
\hline $\mathrm{NaCl}$ & $\mathrm{Na}$ & 1 & $\mathrm{Cl}$ & 1 & & & & & & & & \\
\hline $\mathrm{Mg} / \mathrm{Na} \mathrm{EX}$ & $\mathrm{Na}$ & 2 & $\mathrm{Mg}$ & -1 & & & & & & & & \\
\hline Calcite & $\mathrm{Ca}$ & 1 & $\mathrm{C}$ & 1 & $\mathrm{RS}$ & 4 & I1 & 0 & $\mathrm{I} 2$ & 0 & & \\
\hline "CH2O" & $\mathrm{C}$ & 1 & I1 & -25 & $\mathrm{I} 2$ & 0 & & & & & & \\
\hline Kaolinite & $\mathrm{Al}$ & 2 & $\mathrm{Si}$ & 2 & & & & & & & & \\
\hline Ca-Mont & $\mathrm{Ca}$ & 0.167 & $\mathrm{Al}$ & 2.33 & $\mathrm{Si}$ & 3.67 & & & & & & \\
\hline Na-Mont & $\mathrm{Na}$ & 0.33 & $\mathrm{Al}$ & 2.33 & $\mathrm{Si}$ & 3.67 & & & & & & \\
\hline MgSiO3 & $\mathrm{Mg}$ & 1 & $\mathrm{Si}$ & 1 & & & & & & & & \\
\hline Lignite & $\mathrm{C}$ & 1 & $\mathrm{RS}$ & -0.4 & I1 & -25 & $\mathrm{I} 2$ & 0 & & & & \\
\hline
\end{tabular}

$70 \quad$ models checked

12 models found:

\begin{tabular}{|lcc|}
\hline & \multicolumn{2}{c|}{ Model 1 } \\
Gypsum & $-\mathrm{F}$ & 137.0132 \\
& (Constraint ignored) \\
Dolomite & $-\mathrm{F}$ & -123.35462 \\
$\mathrm{NaCl}$ & $+\mathrm{F}$ & 10.64808 \\
$\mathrm{Mg} / \mathrm{Na} \mathrm{EX}$ & & -215.85055 \\
"CH2O" & + & 257.8056 \\
Kaolinite & & -2798.85488 \\
Na-Mont & & 1525.38627 \\
\hline
\end{tabular}

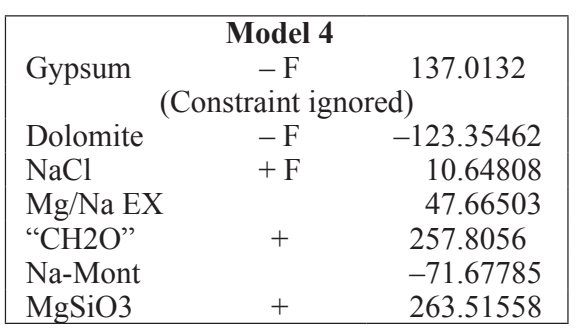

\begin{tabular}{|lcr|}
\hline & \multicolumn{2}{c|}{ Model 7 } \\
Gypsum & $-\mathrm{F}$ & 137.0132 \\
& (Constraint ignored) \\
Dolomite & $-\mathrm{F}$ & -112.42015 \\
$\mathrm{NaCl}$ & $+\mathrm{F}$ & 10.64808 \\
$\mathrm{Mg} / \mathrm{Na} \mathrm{EX}$ & & 35.83818 \\
$\mathrm{Ca}-\mathrm{Mont}$ & & -65.47586 \\
$\mathrm{MgSiO} 3$ & + & 240.75427 \\
Lignite & + & 235.93666 \\
\hline
\end{tabular}

\begin{tabular}{|lcr|}
\hline \multirow{3}{*}{ Gypsum } & $\begin{array}{c}\text { Model 10 } \\
\text { - F }\end{array}$ & 137.0132 \\
& (Constraint ignored) \\
Dolomite & $-\mathrm{F}$ & -79.28608 \\
$\mathrm{NaCl}$ & $+\mathrm{F}$ & 10.64808 \\
"CH2O" & + & 169.66853 \\
$\mathrm{Ca}-\mathrm{M}$ ont & & -263.88345 \\
Na-Mont & & 217.20112 \\
MgSiO3 & + & 171.78201 \\
\hline
\end{tabular}

(ignoring 1 dissolution/precipitation constraint)

\begin{tabular}{|lcc|}
\hline \multicolumn{3}{c|}{ Model 2 } \\
Gypsum & $-\mathrm{F}$ & 137.0132 \\
& (Constraint ignored) \\
Dolomite & $-\mathrm{F}$ & -123.35462 \\
$\mathrm{NaCl}$ & $+\mathrm{F}$ & 10.64803 \\
$\mathrm{Mg} / \mathrm{Na} \mathrm{EX}$ & & 35.83818 \\
"CH2O" & + & 257.8056 \\
Kaolinite & & -125.61544 \\
MgSiO3 & + & 251.68874 \\
\hline
\end{tabular}

\begin{tabular}{|lcr|}
\hline \multicolumn{3}{c|}{ Model 5 } \\
Gypsum & $-\mathrm{F}$ & 137.0132 \\
& (Constraint ignored) \\
Dolomite & $-\mathrm{F}$ & -123.35462 \\
$\mathrm{NaCl}$ & $+\mathrm{F}$ & 10.64808 \\
$\mathrm{Mg} / \mathrm{Na} \mathrm{EX}$ & & -215.85055 \\
Kaolinite & & -2798.85488 \\
Na-Mont & & 1525.38627 \\
Lignite & + & 257.8056 \\
\hline
\end{tabular}

\begin{tabular}{|lcc|}
\hline \multicolumn{3}{|c|}{ Model 8 } \\
Gypsum & $-\mathrm{F}$ & 137.0132 \\
& (Constraint ignored) \\
Dolomite & $-\mathrm{F}$ & -123.35462 \\
$\mathrm{NaCl}$ & $+\mathrm{F}$ & 10.64808 \\
$\mathrm{Mg} / \mathrm{Na} \mathrm{EX}$ & & 47.66503 \\
$\mathrm{Na}-\mathrm{Mont}$ & & -71.67785 \\
$\mathrm{MgSiO} 3$ & + & 263.51558 \\
Lignite & + & 257.8056 \\
\hline
\end{tabular}

\begin{tabular}{|lcc|}
\hline & \multicolumn{2}{c|}{ Model 11 } \\
Gypsum & $-\mathrm{F}$ & 137.0132 \\
& (Constraint ignored) \\
Dolomite & $-\mathrm{F}$ & -123.35462 \\
$\mathrm{NaCl}$ & $+\mathrm{F}$ & 10.64808 \\
Kaolinite & & -506.2604 \\
Na-Mont & & 217.20112 \\
MgSiO3 & + & 215.85055 \\
Lignite & + & 257.8056 \\
\hline
\end{tabular}

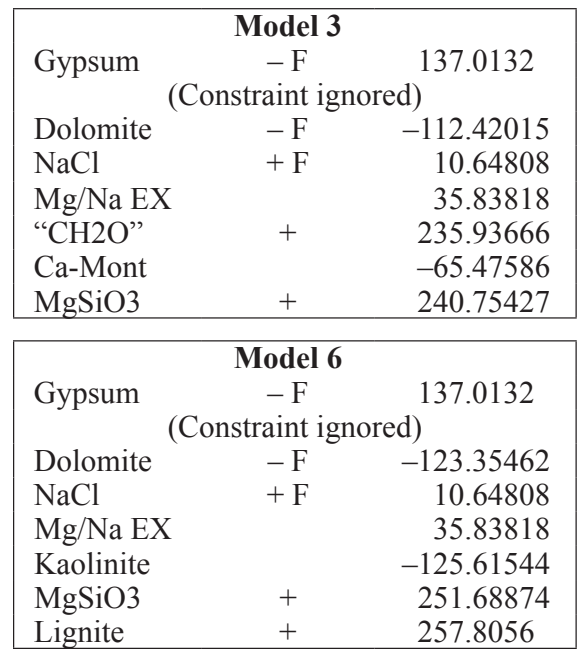

\begin{tabular}{|lcc|}
\hline & \multicolumn{2}{c|}{ Model 9 } \\
Gypsum & $-\mathrm{F}$ & 137.0132 \\
& (Constraint ignored) \\
Dolomite & $-\mathrm{F}$ & -123.35462 \\
$\mathrm{NaCl}$ & $+\mathrm{F}$ & 10.64808 \\
"CH2O" & + & 257.8056 \\
Kaolinite & & -506.2604 \\
Na-Mont & & 217.20112 \\
MgSiO3 & + & 215.85055 \\
\hline
\end{tabular}

\begin{tabular}{|lcr|}
\hline \multirow{3}{*}{ Gypsum } & $\begin{array}{c}\text { Model 12 } \\
-\mathrm{F}\end{array}$ & 137.0132 \\
& \multicolumn{2}{c|}{ (Constraint ignored) } \\
Dolomite & $-\mathrm{F}$ & -79.28608 \\
$\mathrm{NaCl}$ & $+\mathrm{F}$ & 10.64808 \\
$\mathrm{Ca}-\mathrm{Mont}$ & & -263.88345 \\
$\mathrm{Na}-\mathrm{Mont}$ & & 217.20112 \\
MgSiO3 & + & 171.78201 \\
Lignite & + & 169.66853 \\
\hline
\end{tabular}


Publishing support provided by:

Denver Publishing Service Center, Denver, Colorado

For more information concerning this publication, contact: Director, USGS Colorado Water Science Center

Box 25046, Mail Stop 415

Denver, CO 80225

(303) 236-4882

Or visit the Colorado Water Science Center Web site at: http://co.water.usgs.gov/

This publication is available online at: http://dx.doi.org/10.3133/sir20135074 

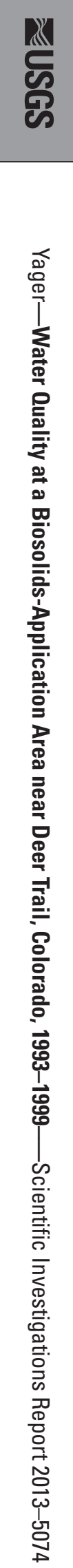

ISSN 2328-0328 (online) 